\title{
Interactive Sensing and Decision Making in Social Networks
}

\author{
Vikram Krishnamurthy \\ Omid Namvar Gharehshiran \\ Maziyar Hamdi \\ University of British Columbia \\ Vancouver, Canada
}

This monograph appears in Foundation and Trends in Signal Processing, DOI: 10.1561/2000000048, Now Publsihers.

(C)2014 Vikram Krishnamurthy, Omid Namvar Gharehshiran and Maziyar Hamdi 


\section{Contents}

Preface 1

1 Introduction and Motivation 3

1.1 Motivation . . . . . . . . . . . . . . . . . . 4

1.2 Main Results and Organization . . . . . . . . . . . . . . 5

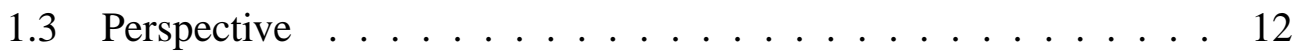

2 Social Learning Approach to Interactive Sensing 15

2.1 Introduction . . . . . . . . . . . . . . . . . . 15

2.2 Multi-Agent Social Learning . . . . . . . . . . . . . . 17

2.2.1 Motivation: What is social learning? . . . . . . . . . 17

2.2 .2 Discussion . . . . . . . . . . . . . . . 19

2.2.3 Rational Agents form Information Cascades . . . . . . . . 20

2.2.4 Individual Privacy vs Group Reputation . . . . . . . . . . 22

2.3 Data Incest in Online Reputation Systems . . . . . . . . . . . 25

2.3.1 Information Exchange Graph . . . . . . . . . . . . . 27

2.3.2 Fair Online Reputation System . . . . . . . . . . . . . 29

2.3.3 Incest Removal Algorithm in Online Reputation System . 33

2.3 .4 Summary . . . . . . . . . . . . . . . . . . . 35

2.4 Interactive Sensing for Quickest Change Detection . . . . . . 36

2.4.1 Classical Quickest Detection . . . . . . . . . . . . . . . . 37

2.4.2 Multi-agent Quickest Detection Problem . . . . . . . . 39

2.5 Closing Remarks . . . . . . . . . . . . . . . . . . 42

3 Tracking Degree Distribution in Dynamic Social Networks

3.1 Introduction . . . . . . . . . . . . . . . . . . . . . 46

3.1 .1 Motivation . . . . . . . . . . . . . . 46

3.1 .2 Main Results . . . . . . . . . . . . . . . . . . 47 
3.1.3 Related Works . . . . . . . . . . . . . . . . 48

3.2 Markov-Modulated Random Graphs . . . . . . . . . . . . . 50

3.3 Degree Distribution Analysis of Dynamic Random Graphs . . . . 53

3.4 Power Law Analysis of Infinite Duplication-deletion Graph . . . . 55

3.5 Social Network as a Social Sensor . . . . . . . . . . . . . . 57

3.5.1 Tracking Error of the Stochastic Approximation Algorithm 59

3.5.2 Limit System Characterization . . . . . . . . . . . . . . 60

3.5.3 Scaled Tracking Error . . . . . . . . . . . . . . . . 61

3.6 A Note on Degree-based Graph Construction . . . . . . . . . 62

3.7 Numerical Examples . . . . . . . . . . . . . . . . . . . . . . . . 65

3.8 Closing Remarks . . . . . . . . . . . . . . . . . . . . . . . . . . . 69

4 Sensing with Information Diffusion in Social Networks 71

4.1 Introduction . . . . . . . . . . . . . . . 71

$4.1 .1 \quad$ Aim . . . . . . . . . . . . . . . . 72

4.1 .2 Motivation . . . . . . . . . . 72

4.1.3 Experimental Studies . . . . . . . . . . . . . . . . . 74

4.2 Social Network Model . . . . . . . . . . . . . . . . . . . . . . 74

4.2.1 Target Dynamics . . . . . . . . . . . . . . . . . 75

4.2.2 Diffusion of Information in Social Network . . . . . . . 75

4.3 Sentiment-Based Sensing Mechanism . . . . . . . . . . . . 79

4.3.1 Uniform Sampling . . . . . . . . . . . . . . . . . 80

4.3.2 Non-Uniform Social Sampling . . . . . . . . . . . . . . 80

4.3.3 MCMC Based Respondent-Driven Sampling (RDS) . . . 81

4.3.4 Extrinsic Observations . . . . . . . . . . . . . . . . . 82

4.4 Mean Field Dynamics for Social Sensing _ . . . . . . . . . 83

4.5 Information Diffusion Matched to Target State Dynamics . . . . . 83

4.5.1 Mean Field Dynamics . . . . . . . . . . . . . . . . . . 84

4.5.2 Sentiment-Based Observations . . . . . . . . . . . . . . 86

4.5.3 Bayesian Filtering Problem . . . . . . . . . . . 87

4.6 Mean Field Dynamics . . . . . . . . . . . . . . . 88

4.6.1 Bayesian Filtering Problem . . . . . . . . . . . . 89

4.6.2 Numerical Example . . . . . . . . . . . . . . . . . . 89

4.7 Closing Remarks . . . . . . . . . . . . . . . . . . . . . . 91

5 Non-Cooperative Game-Theoretic Learning 93

5.1 Introduction . . . . . . . . . . . . . . . . . . . 93

$5.1 .1 \quad$ Literature . . . . . . . . . . . . . . . . 94 
5.1.2 Experimental Studies . . . . . . . . . . . . . 95

5.2 Non-Cooperative Games and Correlated Equilibrium . . . . . . . 97

5.2.1 Strategic Form Non-Cooperative Games . . . . . . . . . . 97

5.2.2 Solution Concepts: Correlated vs. Nash Equilibrium . . . 99

5.3 Local Adaptation and Learning . . . . . . . . . . . . . . . . . . . . . . . . . . . . . .

5.3.1 Case I: Fully Social Players . . . . . . . . . . . . . 105

5.3 .2 Case II: Social Groups . . . . . . . . . . . . . . . . . . . . . . . . . . . . . .

5.3.3 Case III: Homogeneous Social Groups . . . . . . . . . . . . . . 112

5.4 Emergence of Rational Global Behavior . . . . . . . . . . . . . . 115

5.4.1 Global Behavior . . . . . . . . . . . . . 117

5.4.2 Main Result: Convergence to Correlated Equilibrium . . . 117

5.4.3 Sketch of Convergence Proof . . . . . . . . . . . . . . . . 119

5.5 Markov Modulated Non-Cooperative Games . . . . . . . . . . . . 125

5.6 Example: Energy-Aware Sensing via Diffusion LMS . . . . . . . 128

5.6.1 Centralized Parameter Estimation Problem . . . . . . . . 129

5.6.2 Diffusion Least Mean Squares (LMS) . . . . . . . . . . . 130

5.6.3 Energy-Aware Activation Control of Diffusion LMS . . . 132

5.6 .4 Numerical Study . . . . . . . . . . . . . . . . . . 137

5.7 Closing Remarks . . . . . . . . . . . . . . . 138

6 Summary 141

A Appendix to Chapter 3

A.1 Proof of Theorem 3.3.1 . . . . . . . . . . . . . . . . 144

A.2 Proof of Theorem 3.5.1 . . . . . . . . . . . . . . . . . . . 149

A.3 Sketch of the Proof of Theorem 3.5.2 . . . . . . . . . . . . . . . 153

A.4 Sketch of the Proof of Theorem 3.5.3 . . . . . . . . . . 154

B Appendix to Chapter 5

B.1 Proof of Theorem 5.4.2 . . . . . . . . . . . . 157

B.2 Proof of Theorem 5.4.5 . . . . . . . . . . . 161

B.3 Proof of Theorem 5.4.6 ................ 165

$\begin{array}{ll}\text { References } & 167\end{array}$

Index 190 


\section{Preface}

The proliferation of social media such as real time microblogging and online reputation systems facilitate real time sensing of social patterns and behavior. In the last decade, sensing and decision making in social networks have witnessed significant progress in the electrical engineering, computer science, economics, finance, and sociology research communities. Research in this area involves the interaction of dynamic random graphs, socio-economic analysis, and statistical inference algorithms. This monograph provides a survey, tutorial development, and discussion of four highly stylized examples: social learning for interactive sensing; tracking the degree distribution of social networks; sensing and information diffusion; and coordination of decision making via game-theoretic learning. Each of the four examples is motivated by practical examples, and comprises of a literature survey together with careful problem formulation and mathematical analysis. Despite being highly stylized, these examples provide a rich variety of models, algorithms and analysis tools that are readily accessible to a signal processing, control/systems theory, and applied mathematics audience. 


\section{Acknowledgements}

Some of the work in this monograph is based on papers we have co-authored with Professor George Yin, Department of Mathematics, Wayne State University. We are grateful for his expertise in weak convergence analysis of stochastic approximation algorithms. Chapter 2 is based on a recent paper with Professor Vincent Poor, Department of Electrical Engineering, Princeton University. We are also grateful to Professor Christophe Chamley, Department of Economics, Boston University, for discussions that sparked our interest in social learning.

The research in this monograph was supported in part by the the Canada Research Chairs Program, Natural Sciences \& Engineering Research Council of Canada, and Social Sciences \& Humanities Research Council of Canada. 


\section{Chapter 1}

\section{Introduction and Motivation}

Research in social networks involves the interplay of complex networks (dynamics of random graphs) and social analysis (stemming from the areas of economics and sociology). There are seminal books in this area including [132, 249]. In comparison, this monograph deals with sensing and decision-making in social networks. The proliferation of social media such as real-time microblogging services (Twitter 1 ), online reputation and rating systems (Yelp) together with app-enabled smartphones, facilitate real time sensing of social activities, social patterns, and behavior.

Sensing and decision making in social networks is an area that has witnessed remarkable progress in the last decade in electrical engineering, computer science, economics, finance, and sociology. It is the aim of this monograph to survey some important topics in this area and present highly stylized examples that are readily accessible to a signal processing, control/systems theory, and applied mathematics audience. Indeed, the main tools used in this monograph are dynamic programming, Bayesian estimation (filtering), stochastic approximation (adaptive filtering) and their convergence analysis (weak convergence and mean square analysis), game-theoretic learning, and graph theory. There has been much recent activity in the signal processing community in the area of social networks. "How global behavior emerges from simple local behavior of boundedly rational agents" has been an underlying theme of an NSF/UCLA workshop in 2010, special sessions at ICASSP 2011 and 2012 and the ICASSP 2011 expert summary in [270]. Also, the recent special issues [227, 268] deal with signal processing of social networks.

\footnotetext{
${ }^{1}$ On US Presidential election day in 2012, there were 15 thousand tweets per second resulting in 500 million tweets in the day. Twitter can be considered as a real-time sensor.
} 


\subsection{Motivation}

Social sensing [5, 41, 44, 75] is defined as a process where physical sensors present in mobile devices such as GPS are used to infer social relationships and human activities. In this monograph, we work at a higher level of abstraction. We use the term social sensor or human-based sensor to denote an agent that provides information about its environment (state of nature) on a social network after interaction with other agents. Examples of such social sensors include Twitter posts, Facebook status updates, and ratings on online reputation systems like Yelp and Tripadvisor. Such social sensors go beyond physical sensors for social sensing [221]. For example, user opinions/ratings (such as the quality of a restaurant) are available on Tripadvisor but are difficult to measure via physical sensors. Similarly, future situations revealed by the Facebook status of a user are impossible to predict using physical sensors.

Statistical inference using social sensors is relevant in a variety of applications including localizing special events for targeted advertising [59, 171], marketing [245], localization of natural disasters [222], and predicting sentiment of investors in financial markets [33, 208]. It is demonstrated in [13] that models built from the rate of tweets for particular products can outperform market-based predictors. However, social sensors present unique challenges from a statistical estimation point of view. First, social sensors interact with and influence other social sensors. For example, ratings posted on online reputation systems strongly influence the behaviour of individuals 2 . Such interactive sensing can result in non-standard information patterns due to correlations introduced by the structure of the underlying social network. Second, due to privacy reasons and time constraints, social sensors typically do not reveal raw observations of the underlying state of nature. Instead, they reveal their decisions (ratings, recommendations, votes) which can be viewed as a low resolution (quantized) function of their raw measurements and interactions with other social sensors.

As is apparent from the above discussion, there is strong motivation to construct mathematical models that capture the dynamics of interactive sensing involving social sensors. Such models facilitate understanding the dynamics of information flow in social networks and, therefore, the design of algorithms that can exploit these dynamics to estimate the underlying state of nature. In this monograph, social learning [23, 31, 50], game-theoretic learning [92, 121], and

\footnotetext{
${ }^{2}$ It is reported in [130] that $81 \%$ of hotel managers regularly check Tripadvisor reviews. It is reported in [187] that a one-star increase in the Yelp rating maps to 5-9\% revenue increase.
} 


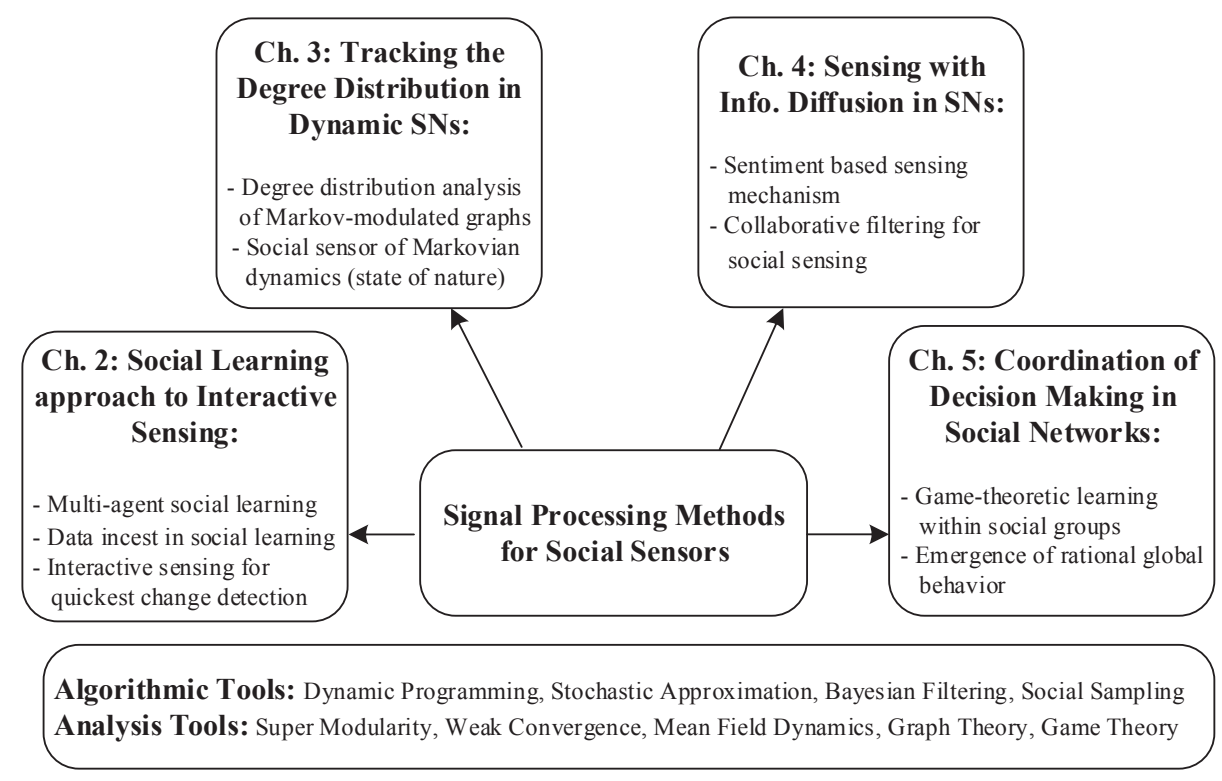

Figure 1.1: Main results and organization of the monograph.

stochastic approximation [167, 263] serve as useful mathematical abstractions for modelling the interaction of social sensors.

\subsection{Main Results and Organization}

As can be seen from Figure 1.1, this monograph is organized into four chapters (excluding this introductory chapter) that provide a survey, tutorial development, and discussion of four highly stylized examples: social learning for interactive sensing; tracking the degree distribution of social networks; sensing and information diffusion; and coordination of decision-making via game-theoretic learning. Each of the four chapters is motivated by practical examples, and comprises of a literature survey together with careful problem formulation and mathematical analysis. The examples and associated analysis are readily accessible to a signal processing, control/systems theory, and applied mathematics audience.

In terms of information patterns, Chapter 2 considers Bayesian estimation and sequential decision making with sequential information flow and then informa- 
tion flow over small directed acyclic graphs. In comparison, Chapter 3 considers stochastic approximation algorithms for large random graphs that evolve with time. Chapter 4 considers the asymptotics of large graphs with fixed degree distribution but where the state of individual node in the graph evolve over time - this models information diffusion. The mean field analysis in Chapter 4 results in a stochastic approximation type recursion, and the estimation problems are Bayesian (nonlinear filtering). Finally, Chapter 5 deals with learning in non-cooperative repeated games comprising networks of arbitrary size-the algorithms are of the stochastic approximation type. In all these cases, sensors interact with and influence other sensors. It is the understanding of this interaction of local and global behaviors in the context of social networks that constitutes the unifying theme of this monograph.

Below we give a brief synopsis of these four chapters.

\section{Social Learning Approach to Interactive Sensing}

Chapter 2 presents models and algorithms for interactive sensing in social networks where individuals act as sensors and the information exchange between individuals is exploited to optimize sensing. Social learning is used as a mathematical formalism to model the interaction between individuals that aim to estimate an underlying state of nature.

Social learning in multi-agent systems seeks to answer the following question:

How do decisions made by agents affect decisions made by subsequent agents?

In social learning, each agent chooses its action by optimizing its local utility function. Subsequent agents then use their private observations together with the decisions of previous agents to estimate (learn) the underlying state of nature. The setup is fundamentally different to classical signal processing in which sensors use noisy observations to compute estimates.

In the last decade, social learning has been used widely in economics, marketing, political science, and sociology to model the behavior of financial markets, crowds, social groups, and social networks; see [1, 2, 23, 31, 50, 180] and numerous references therein. Related models have been studied in the context of sequential decision making in information theory [65, 126] and statistical signal processing [51, 162] in the electrical engineering literature.

Social learning models for interactive sensing can predict unusual behavior. Indeed, a key result in social learning of an underlying random variable is that 
rational agents eventually herd [31]; that is, they eventually end up choosing the same action irrespective of their private observations. As a result, the actions contain no information about the private observations and so the Bayesian estimate of the underlying random variable freezes. For a multi-agent sensing system, such behavior can be undesirable, particularly if individuals herd and make incorrect decisions.

In this context, the following questions are addressed in Chapter 2, How can self-interested agents that interact via social learning achieve a trade-off between individual privacy and reputation of the social group? How can protocols be designed to prevent data incest in online reputation blogs where individuals make recommendations? How can sensing by individuals that interact with each other be used by a global decision maker to detect changes in the underlying state of nature? Chapter 2 presents an overview, insights and discussion of social learning models in the context of data incest propagation, change detection, and coordination of decision making.

Several examples in social networks motivate Chapter 2, Design of protocols to prevent data incest are motivated by the design of fair online reputation systems such as Yelp or Tripadvisor. In Online reputation systems, which maintain logs of votes (actions) by agents, social learning takes place with information exchange over a loopy graph (where the agents form the vertices of the graph). Due to the loops in the information exchange graph, data incest (misinformation) can propagate: Suppose an agent wrote a poor rating of a restaurant on a social media site. Another agent is influenced by this rating, visits the restaurant, and then also gives a poor rating on the social media site. The first agent visits the social media site and notices that another agent has also given the restaurant a poor rating-this double confirms her rating and she enters another poor rating. In a fair reputation system, such "double counting" or data incest should have been prevented by making the first agent aware that the rating of the second agent was influenced by her own rating.

As an example of change detection, consider measurement of the adoption of a new product using a micro-blogging platform like Twitter. The adoption of the technology diffuses through the market but its effects can only be observed through the tweets of select members of the population. These selected members act as sensors for the parameter of interest. Suppose the state of nature suddenly changes due to a sudden market shock or presence of a new competitor. Based on the local actions of the multi-agent system that is performing social learning, a global decision maker (such as a market monitor or technology manufacturer) needs to decide whether or not to declare if a change has occurred. How can the 
global decision maker achieve such change detection to minimize a cost function comprised of false alarm rate and delay penalty? The local and global decision makers interact, since the local decisions determine the posterior distribution of subsequent agents which determines the global decision (stop or continue) which determines subsequent local decisions.

\section{Tracking Degree Distribution of Social Networks}

Chapter 3 considers dynamical random graphs. The degree of a node in a network (also known as the connectivity) is the number of connections the node has in that network. The most important measure that characterizes the structure of a network (specially when the size of the network is large and the connections-adjacency matrix of the underlying graph-are not given) is the degree distribution of the network. Chapter 3 considers a Markov-modulated duplication-deletion random graph where, at each time instant, one node can either join or leave the network with probabilities that evolve according to the realization of a finite state Markov chain (state of nature). This chapter deals with the following questions:

How can one estimate the state of nature using noisy observations of nodes' degrees in a social network? and How good are these estimates?

Chapter 3 comprises of two results. First, motivated by social network applications, we analyze the asymptotic behavior of the degree distribution of the Markov-modulated random graph. From this degree distribution analysis, we can study the connectivity of the network, the size and the existence of a large connected component, the delay in searching such graphs, etc. [86, 132, 204, 202]. Second, a stochastic approximation algorithm is presented to track the empirical degree distribution as it evolves over time. We further show that the stationary degree distribution of Markov-modulated duplication-deletion random graphs depends on the dynamics of such graphs and, thus, on the state of nature. This means that, by tracking the empirical degree distribution, the social network can be viewed as a social sensor to track the state of nature. The tracking performance of the algorithm is analyzed in terms of mean square error. A functional central limit theorem is further presented for the asymptotic tracking error.

An important associated problem discussed in Chapter 3 is how to actually construct random graphs via simulation algorithms. In particular, for large social networks, only the degree sequence is available, and not the adjacency matrix. (The degree sequence is a non-increasing sequence of vertex degrees.) Does a 
simple graph exist that realizes a particular degree sequence? How can all graphs that realize a degree sequence be constructed? Chapter 3 presents a discussion of these issues.

\section{Sensing and Information Diffusion in Social Networks}

Chapter 4 considers the following questions:

How does a behavior diffuse over a social network comprising of a population of interacting agents? and How can an underlying stochastic state be estimated based on sampling the population?

As described in [184], there is a wide range of social phenomena such as diffusion of technological innovations, cultural fads, and economic conventions [50], where individual decisions are influenced by the decisions of others. Chapter 4 considers two extensions of the widely used Susceptible-Infected-Susceptible (SIS) models for diffusion of information in social networks [183, 184, 132, 212, 249]. First, the states of individual nodes evolve over time as a probabilistic function of the states of their neighbors and an underlying target process. The underlying target process can be viewed as the market conditions or competing technologies that evolve with time and affect the information diffusion. Second, the nodes in the social network are sampled randomly to determine their state. Chapter 4 reviews recent methods for sampling social networks such as social sampling and respondentdriven sampling. As the adoption of the new technology diffuses through the network, its effect is observed via sentiment (such as tweets) of these selected members of the population. These selected nodes act as social sensors. In signal processing terms, the underlying target process can be viewed as a signal, and the social network can be viewed as a sensor. The key difference compared to classical signal processing is that the social network (sensor) has dynamics due to the information diffusion. Our aim is to estimate the underlying target state and the state probabilities of the nodes by sampling measurements at nodes in the social network. In a Bayesian estimation context, this is equivalent to a filtering problem involving estimation of the state of a prohibitively large-scale Markov chain in noise. The key idea is to use mean field dynamics as an approximation (with provable bounds) for the information diffusion and, thereby, obtain a tractable model. 


\section{Coordination of Decisions as Non-cooperative Game-Theoretic Learning}

Chapter 5 studies game-theoretic learning in the context of social networks. Game theory has traditionally been used in economics and social sciences with a focus on fully rational interactions where strong assumptions are made on the information patterns available to individual agents. In comparison, social sensors are agents with partial information and it is the dynamic interactions among such agents that is of interest. This, together with the interdependence of agents' choices, motivates the need for game-theoretic learning models for agents interacting in social networks.

Chapter 5 deals with the question:

When individuals are self-interested and possess limited sensing and communication capabilities, can a network of such individuals achieve sophisticated global behavior?

We discuss a non-cooperative game-theoretic learning approach for adaptive decision making in social networks. This can be viewed as non-Bayesian social learning. The aim is to ensure that all agents eventually choose actions from a common polytope of randomized strategies - namely, the set of correlated equilibria [18, 20] of a non-cooperative game. The game-theoretic concept of equilibrium describes a condition of global coordination where all decision makers are content with the social welfare realized as the consequence of their chosen strategies.

We consider two examples of information exchange among individuals. The first example comprises of fully social agents that can communicate with every other agent in the network. This provides a simple framework to present the "regret-matching" [117, 121] decision making procedure that ensures convergence of the global behavior of the network to the correlated equilibria set. In the second example, we confine the information flow to social groups-each individual can only speak with her neighbors. Accordingly, the regret-matching procedure is revised to adapt to this more practical social network model. Finally, we consider the case of homogeneous social groups, where individuals share and are aware of sharing the same incentives. The regret-matching procedures is then adapted to exploit this valuable piece of information available to individuals within each social group. The final result in this chapter considers the scenario where the non-cooperative game model evolves with time according to the sample path of a finite-state Markov chain. It is shown that, if the speed of the Markovian jumps and the learning rate of the regret-matching algorithm match, the global be- 
havior emerging from a network of individuals following the presented algorithms properly tracks the time-varying set of correlated equilibria.

One of the main ideas in this chapter is that the limit system that represents the game-theoretic learning algorithms constitutes a differential inclusion. Differential inclusions are generalization of ordinary differential equations (ODEs) 3 , and arise naturally in game-theoretic learning, since the strategies according to which others play are unknown. This is highly non-standard in the analysis of stochastic approximation algorithms in which the limit system is usually an ODE.

Chapter 5 ends with an example that shows how the presented algorithms can be applied in a social sensing application. We consider the problem of estimating the true value of an unknown parameter via a network of sensors. There have been a lot of recent works that study diffusion of information over graphs linking a multitude of agents; see [226, 225] and numerous references therein. We particulary focus on diffusion least mean square (LMS) algorithms [182]: each sensor decides whether to activate, and if activates, (i) it will exchange estimate with neighbors and fuse the collected data; (ii) it will use the fused data and local measurements to refine its estimate via an LMS-type adaptive filter. Using a game-theoretic formulation, an energy-aware activation mechanism is devised that, taking into account the spatial-temporal correlation of sensors' measurements, prescribes sensors when to activate. We first show that, as the step-size in the diffusion LMS approaches zero, the analysis falls under the unifying classical stochastic approximation theme of this chapter and, therefore, can be done using the well-known ODE method [167]. It is then shown that the proposed algorithm ensures the estimate at each sensor converges to the true parameter, yet the global activation behavior along the way tracks the set of correlated equilibria of the underlying activation control game.

\section{Appendices}

The two appendices at the end of this monograph present, respectively, a meansquare error analysis and weak convergence analysis of two different types of stochastic approximation algorithms used to track time-varying behavior in social networks. These analysis are crucial in allowing us to predict the asymptotic dynamics of such algorithms. The chapters provide sufficient intuition behind the theorems and the reader can skip the appendices without loss of continuity.

\footnotetext{
${ }^{3} \mathrm{~A}$ generic differential inclusion is of the form $d X / d t \in \mathcal{F}(X, t)$, where $\mathcal{F}(X, t)$ specifies a family of trajectories rather than a single trajectory as in the ordinary differential equations $d X / d t=F(X, t)$. See 5.4 .3 and Appendix $\mathrm{B}$ for more details.
} 
Appendix A.1 generalizes the asymptotic analysis of duplication deletion random graphs in [61] to the case of Markov-modulated graphs. It uses the concept of perturbed Lyapunov functions. The weak convergence analysis presented in Appendix B generalizes the convergence analysis provided in the seminal papers [117, 119] to the case where the game-theoretic learning algorithm can track a time-varying correlated equilibrium. The convergence analysis in both appendices are presented in a tutorial fashion and are readily accessible to researchers in adaptive filtering, stochastic optimization, and game theory.

\section{Out-of-Scope Topics}

Other important problems that have been extensively studied in the literature, but are outside the scope of this monograph include: consensus formation [132, Chapter 8], [149, 240], metrics for measuring networks (other than degree distribution) [132, Chapter 2], [253], small world [144, 254, 255], cooperative models of network formation [131, Chapter 1], [132, Chapter 12], [233], behavior dynamics in peer-to-peer media-sharing social networks [112, 267], and privacy and security modeling [169, 179]. The interested reader is referred to the above cited works and references therein for extensive treatment of the topics.

\subsection{Perspective}

The social learning and game-theoretic learning formalisms considered in this monograph can be used either as descriptive tools, to predict the outcome of complex interactions amongst agents in sensing, or as prescriptive tools, to design social networks and sensing systems around given interaction rules. Information aggregation, misinformation propagation and privacy are important issues in sensing using social sensors. In this monograph, we treat these issues in a highly stylized manner so as to provide easy accessibility to an electrical engineering audience. The underlying tools used in this monograph are widely used by the electrical engineering research community in the areas of signal processing, control, information theory and network communications. The fundamental theory of network science is well-documented in seminal books such as [76, 132] and involves the interplay of random graphs and game theory.

In Bayesian estimation, the twin effects of social learning (information aggregation with interaction amongst agents) and data incest (misinformation propagation) lead to non-standard information patterns in estimating the underlying 
state of nature. Herding occurs when the public belief overrides the private observations and, thus, actions of agents are independent of their private observations. Data incest results in bias in the public belief as a consequence of the unintentional re-use of identical actions in the formation of public belief in social learning - the information gathered by each agent is mistakenly considered to be independent. This results in overconfidence and bias in estimates of the state of nature.

Tracking a time-varying parameter that evolves according to a finite-state Markov chain (state of nature) is a problem of much interest in signal processing [30, 87, 259]. In social networks, sometimes the parameter under study (state of nature) cannot be sensed by pervasive sensors, e.g., the level of happiness in a community, the tendency of individuals to expand their networks, the strength of social links between individuals, etc. In such cases, social sensors can do much better than pervasive sensors. A social network with a large number of individuals can be viewed as an interactive sensing tool to obtain information about individuals or state of nature; this is a social sensor. Motivated by social network applications, a social sensor based framework is presented in Chapter 3 to track the degree distribution of Markov-modulated dynamic networks whose dynamics evolve over time according to a finite-state Markov chain.

Privacy issues impose important constraints on social sensors. Typically, individuals are not willing to disclose private observations. Optimizing interactive sensing with privacy constraints is an important problem. Privacy and trust pose conflicting requirements on human-based sensing: Privacy requirements result in noisier measurements or lower resolution actions, while maintaining a high degree of trust (reputation) requires accurate measurements. Utility functions, noisy private measurements, and quantized actions are essential ingredients of the social and game-theoretic learning models presented in this monograph that facilitate modelling this trade-off between reputation and privacy.

In social sensor systems, the behavior is driven by the actions of a large number of autonomous individuals, who are usually self-interested and optimize their respective objectives. Often, these individuals form social contacts (i.e. links) by choice, rather than by chance. Further, there are always social and economic incentives associated with forming such social contacts based on the information obtained about the state of the nature or contribution to the diffusion of information across the network. The social network analysis using the common graph-theoretic techniques, however, fails to capture the behavior of such selfinterested individuals and the dynamics of their interaction. This motivates the use of game-theoretic methods. Game-theoretic learning explains how coordination in the decisions of such self-interested individuals might arise as a conse- 
quence of a long-run process of adaptation and learning in an interactive environment [94]. Interestingly enough, while each individual has limitations in sensing and communication, the coordinated behavior amongst individuals can lead to the manifestation of sophisticated behavior at the network level.

The literature in the areas of social learning, sensing, and networking is extensive. In each of the following chapters, we provide a brief review of relevant works together with references to experimental data. The book [50] contains a complete treatment of social learning models with several remarkable insights. For further references, we refer the reader to [151, 153, 154, 161, 191]. In [116], a nice description is given of how, if individual agents deploy simple heuristics, the global system behavior can achieve "rational" behavior. The related problem of achieving coherence (i.e., agents eventually choosing the same action or the same decision policy) among disparate sensors of decision agents without cooperation has also witnessed intense research; see [215] and [251]. Non-Bayesian social learning models are also studied in [79, 80].

There is also a growing literature dealing with the interplay of technological networks and social networks [55]. For example, social networks overlaid on technological networks account for a significant fraction of Internet use. Indeed, as discussed in [55], three key aspects of that cut across social and technological networks are the emergence of global coordination through local actions, resource sharing models and the wisdom of crowds (diversity and efficiency gains). These themes are addressed in the current paper in the context of social learning. 


\section{Chapter 2}

\section{Social Learning Approach to Interactive Sensing}

\subsection{Introduction}

This chapter comprises of three parts1:

1. Survey and Review of Social Learning: Section 2.2 presents a brief description of the classical social learning model. We use social learning as the mathematical basis for modelling interaction of social sensors. A key result in social learning is that rational agents eventually herd, that is, they pick the same action irrespective of their private observation and social learning stops. To delay the effect of herding, and thereby enhance social learning, Chamley [50] (see also [235] for related work) has proposed a novel constrained optimal social learning protocol. We describe how self-interested agents performing social learning can achieve useful behavior in terms of optimizing a social welfare function. Such problems are motivated by privacy issues in sensing. If an agent reveals less information in its decisions, it maintains its privacy; on the other hand, as part of a social group, it has an incentive to optimize a social welfare function that helps estimate the state of nature. We review this protocol which is formulated as a sequential stopping time problem. We show that the constrained optimal social learning proposed by Chamley [50] has a threshold switching curve in the space of public belief states. Thus, the global decision to stop can be implemented efficiently in a social learning model.

\footnotetext{
${ }^{1}$ The chapter is an extended version of the paper [163].
} 
2. Data Incest in Online Reputation Systems: Section 2.3 deals with the question: How can data incest (misinformation propagation) be prevented in online reputation blogs where social sensors make recommendations?

In the classical social learning model, each agent acts once in a pre-determined order. However, in online reputation systems such as Yelp or Tripadvisor, which maintain logs of votes (actions) by agents, social learning takes place with information exchange over a loopy graph (where the agents form the vertices of the graph). Due to the loops in the information exchange graph, data incest (misinformation) can propagate: Suppose an agent wrote a poor rating of a restaurant on a social media site. Another agent is influenced by this rating, visits the restaurant, and then also gives a poor rating on the social media site. The first agent visits the social media site and notices that another agent has also given the restaurant a poor rating - this double confirms her rating and she enters another poor rating.

In a fair reputation system, such "double counting" or data incest should have been prevented by making the first agent aware that the rating of the second agent was influenced by her own rating. Data incest results in a bias in the estimate of the state of nature. How can automated protocols be designed to prevent data incest, and thereby maintain a fair online reputation system? Section 2.3 describes how the administrator of a social network can maintain an unbiased (fair) reputation system.

3. Interaction of Local and Global Decision Makers for Change Detection: Section 2.4 deals with the question: In sensing, where individual agents perform social learning to estimate an underlying state of nature, how can changes in the state of nature be detected? Such sensing problems arise in a variety of applications such as financial trading, where individuals react to financial shocks [21]; marketing and advertising [183, 184], where consumers react to a new product; and localization of natural disasters (earthquakes and typhoons) [222].

Consider measurement of the adoption of a new product using a micro-blogging platform like Twitter. The new technology diffuses through the market but its effects can only be observed through the tweets of select members of the population. Suppose the state of nature suddenly changes due to a sudden market shock or presence of a new competitor. Based on the local actions of the multi-agent system that is performing social learning, a global decision maker (such as a market monitor or technology manufacturer) needs to decide whether or not to declare if a change has occurred. How can the global decision maker achieve such change detection to minimize a cost function comprised of false alarm rate and delay penalty? The local and global decision makers interact, since the local decisions determine the posterior distribution of subsequent agents which determines the 
global decision (stop or continue), which in turn determines subsequent local decisions. We show that this social learning based change detection problem leads to unusual behavior: The optimal decision policy of the stopping time problem has multiple thresholds. This is unusual since, if it is optimal to declare that a change has occurred based on the posterior probability of change, it may not be optimal to declare a change when the posterior probability of change is higher!

\subsection{Multi-Agent Social Learning}

This section starts with a brief description of the classical social learning model. We review Chamley's novel constrained optimal social learning protocol, which is formulated as a sequential stopping time problem, and delays the effect of herding [50, 235].

\subsubsection{Motivation: What is social learning?}

We start with a brief description of the 'vanilla' social learning model]. In social learning [50], agents estimate the underlying state of nature not only from their local measurements, but also from the actions of previous agents. (These previous actions were taken by agents in response to their local measurements; therefore, these actions convey information about the underlying state). As we will describe below, the state estimation update in social learning has a drastically different structure compared to the standard optimal filtering recursion and can result in unusual behavior.

Consider a countable number of agents performing social learning to estimate the state of an underlying finite-state Markov chain $x$. Let $\mathbb{X}=\{1,2, \ldots, X\}$ denote a finite state space, $P$ the transition matrix and $\pi_{0}$ the initial distribution of the Markov chain.

Each agent acts once in a predetermined sequential order indexed by $k=$ $1,2, \ldots$. The index $k$ can also be viewed as the discrete time instant when agent $k$ acts. A multi-agent system seeks to estimate $x_{k}$. Assume at the beginning of

\footnotetext{
${ }^{2}$ In typical formulations of social learning, the underlying state is assumed to be a random variable and not a Markov chain. Our description below is given in terms of a Markov chain since we wish to highlight the unusual structure of the social learning filter to a signal processing reader who is familiar with basic ideas in Bayesian filtering. Also, we are interested in change detection problems in which the change time distribution can be modelled as the absorption time of a Markov chain.
} 
iteration $k$, all agents have access to the public belief $\pi_{k-1}$ defined in Step (iv) below. The social learning protocol proceeds as follows [31, 50]:

(i) Private Observation: At time $k$, agent $k$ records a private observation $y_{k} \in$ $\mathbb{Y}$ from the observation distribution $B_{i y}=\mathbb{P}(y \mid x=i), i \in \mathbb{X}$. Throughout this chapter, we assume that $\mathbb{Y}=\{1,2, \ldots, Y\}$ is finite.

(ii) Private Belief: Using the public belief $\pi_{k-1}$ available at time $k-1$ (defined in Step (iv) below), agent $k$ updates its private posterior belief $\eta_{k}(i)=$ $\mathbb{P}\left(x_{k}=i \mid a_{1}, \ldots, a_{k-1}, y_{k}\right)$ as the following Bayesian update (this is the classical Hidden Markov Model filter [81]):

$$
\eta_{k}=\frac{B_{y_{k}} P^{\prime} \pi}{\mathbf{1}_{X}^{\prime} B_{y} P^{\prime} \pi}, \quad B_{y_{k}}=\operatorname{diag}\left(\mathbb{P}\left(y_{k} \mid x=i\right), i \in \mathbb{X}\right) .
$$

Here, $\mathbf{1}_{X}$ denotes the $X$-dimensional vector of ones, $\eta_{k}$ is an $X$-dimensional probability mass function (pmf), and $P^{\prime}$ denotes transpose of the matrix $P$.

(iii) Myopic Action: Agent $k$ takes action $a_{k} \in \mathcal{A}=\{1,2, \ldots, A\}$ to minimize its expected cost

$$
a_{k}=\underset{a \in \mathcal{A}}{\operatorname{argmin}} \mathbf{E}\left\{c(x, a) \mid a_{1}, \ldots, a_{k-1}, y_{k}\right\}=\underset{a \in \mathcal{A}}{\operatorname{argmin}}\left\{c_{a}^{\prime} \eta_{k}\right\} .
$$

Here $c_{a}=(c(i, a), i \in \mathbb{X})$ denotes an $X$-dimensional cost vector, and $c(i, a)$ denotes the cost incurred when the underlying state is $i$ and the agent chooses action $a$.

Agent $k$ then broadcasts its action $a_{k}$ to subsequent agents.

(iv) Social Learning Filter: Given the action $a_{k}$ of agent $k$ and the public belief $\pi_{k-1}$, each subsequent agent $k^{\prime}>k$ computes the public belief $\pi_{k}$ according to the following "social learning filter":

$$
\pi_{k}=T\left(\pi_{k-1}, a_{k}\right), \text { where } T(\pi, a)=\frac{R_{a}^{\pi} P^{\prime} \pi}{\sigma(\pi, a)}
$$

and $\sigma(\pi, a)=\mathbf{1}_{X}^{\prime} R_{a}^{\pi} P^{\prime} \pi$ is the normalization factor of the Bayesian update. In (2.3), the public belief $\pi_{k}(i)=\mathbb{P}\left(x_{k}=i \mid a_{1}, \ldots a_{k}\right)$, and $R_{a}^{\pi}=$ $\operatorname{diag}(\mathbb{P}(a \mid x=i, \pi), i \in \mathbb{X})$ has elements

$$
\begin{aligned}
& \mathbb{P}\left(a_{k}=a \mid x_{k}=i, \pi_{k-1}=\pi\right)=\sum_{y \in \mathbb{Y}} \mathbb{P}(a \mid y, \pi) \mathbb{P}\left(y \mid x_{k}=i\right) \\
& \mathbb{P}\left(a_{k}=a \mid y, \pi\right)=\left\{\begin{array}{l}
1, \text { if } c_{a}^{\prime} B_{y} P^{\prime} \pi \leq c_{\widetilde{a}}^{\prime} B_{y} P^{\prime} \pi, \widetilde{a} \in \mathcal{A}, \\
0, \text { otherwise. }
\end{array}\right.
\end{aligned}
$$


The derivation of the social learning filter (2.3) is given in the discussion below.

\subsubsection{Discussion}

Let us pause to give some intuition about the above social learning protocol.

1. Information Exchange Structure: The information exchange in the above social learning protocol is sequential. Agents send their hard decisions (actions) to subsequent agents. Further, we have assumed that each agent acts once. Another way of viewing the social learning protocol is that there are finitely many agents that act repeatedly in some pre-defined order. If each agent chooses its local decision using the current public belief, the setting will be identical to the social learning setup. We also refer the reader to [2] for several recent results in social learning over several types of network adjacency matrices.

2. Filtering with Hard Decisions: Social learning can be viewed as agents making hard decision estimates at each time and sending these estimates to subsequent agents. In conventional Bayesian state estimation, a soft decision is made, namely, the posterior distribution (or equivalently, observation) that is sent to subsequent agents. For example, if $\mathcal{A}=\mathbb{X}$ and the costs are chosen as $c_{a}=-e_{a}$ where $e_{a}$ denotes the unit indicator with 1 in the $a$-th position, then $\operatorname{argmin}_{a} c_{a}^{\prime} \pi=$ $\operatorname{argmax}_{a} \pi(a)$, i.e., the maximum aposteriori probability (MAP) state estimate. For this example, social learning is equivalent to agents sending the hard MAP estimates to subsequent agents.

Note that rather than sending a hard decision estimate, if each agent chooses its action $a_{k}=y_{k}$ (that is, agents send their private observations), the right-hand side of (2.4) becomes $\sum_{y \in \mathbb{Y}} I\left(y=y_{k}\right) \mathbb{P}\left(y \mid x_{k}=i\right)=\mathbb{P}\left(y_{k} \mid x_{k}=i\right)$ and, thus, the problem becomes a standard Bayesian filtering problem.

It is also important to note that the filtered distributions obtained via social learning are not commutative in the actions. That is $P\left(x \mid a_{1}=a, a_{2}=\bar{a}\right) \neq$ $P\left(x \mid a_{1}=\bar{a}, a_{2}=a\right)$. In comparison, for Bayesian estimation of random variable $x$ with conditionally independent observations, $P\left(x \mid y_{1}=y, y_{2}=\bar{y}\right) \neq P\left(x \mid y_{1}=\right.$ $\left.\bar{y}, y_{2}=y\right)$.

3. Dependence of Observation Likelihood on Prior: The most unusual feature of the above protocol (to a signal processing audience) is the social learning filter (2.3). In standard state estimation via a Bayesian filter, the observation likelihood given the state is completely parameterized by the observation noise distribution and is functionally independent of the current prior distribution. In the social learning filter, the likelihood of the action given the state (which is denoted 
by $R_{a}^{\pi}$ ) is an explicit function of the prior $\pi$ ! Not only does the action likelihood depend on the prior, but it is also a discontinuous function, due to the presence of the argmin in (2.2).

4. Derivation of Social Learning Filter: The derivation of the social learning filter (2.3) is as follows: Define the posterior as $\pi_{k}(j)=\mathbb{P}\left(x_{k}=j \mid a_{1}, \ldots, a_{k}\right)$. Then,

$$
\begin{aligned}
& \pi_{k}(j)=\frac{1}{\sigma\left(\pi_{k-1}, a_{k}\right)} \mathbb{P}\left(a_{k} \mid x_{k}=j, a_{1}, \ldots, a_{k-1}\right) \\
& \quad \times \sum_{i} \mathbb{P}\left(x_{k}=j \mid x_{k-1}=i\right) \mathbb{P}\left(x_{k-1}=i \mid a_{1}, \ldots, a_{k-1}\right) \\
& =\frac{1}{\sigma\left(\pi_{k-1}, a_{k}\right)} \sum_{y} \mathbb{P}\left(a_{k} \mid y_{k}=y, a_{1}, \ldots, a_{k-1}\right) \\
& \quad \times \mathbb{P}\left(y_{k}=y \mid x_{k}=j\right) \sum_{i} \mathbb{P}\left(x_{k}=j \mid x_{k-1}=i\right) \pi_{k-1}(i) \\
& =\frac{1}{\sigma\left(\pi_{k-1}, a_{k}\right)} \sum_{y} \mathbb{P}\left(a_{k} \mid y_{k}=y, \pi_{k-1}\right) \mathbb{P}\left(y_{k}=y \mid x_{k}=j\right) \sum_{i} P_{i j} \pi_{k-1}(i)
\end{aligned}
$$

where the normalization term is

$$
\begin{array}{rl}
\sigma\left(\pi_{k-1}, a_{k}\right)=\sum_{j} \sum_{y} & \mathbb{P}\left(a_{k} \mid y_{k}=y, \pi_{k-1}\right) \\
\times & \mathbb{P}\left(y_{k}=y \mid x_{k}=j\right) \sum_{i} P_{i j} \pi_{k-1}(i) .
\end{array}
$$

The above social learning protocol and social learning filter(2.3) result in interesting dynamics in state estimation and decision making. We will illustrate two interesting consequences that are unusual to an electrical engineering audience:

- Rational Agents form herds and information cascades and blindly follow previous agents. This is discussed in $\$ 2.2 .3$ below.

- Making global decisions on change detection in a multi-agent system performing social learning results in multi-threshold behavior. This is discussed in $\$ 2.4$ below.

\subsubsection{Rational Agents form Information Cascades}

The first consequence of the unusual nature of the social learning filter $(2.3)$ is that social learning can result in multiple rational agents taking the same action 
independently of their observations.

Throughout this subsection, we assume that $x$ is a finite state random variable (instead of a Markov chain) with prior distribution $\pi_{0}$. We start with the following definitions; see also [50]:

- An individual agent $k$ herds on the public belief $\pi_{k-1}$ if it chooses its action $a_{k}=a\left(\pi_{k-1}, y_{k}\right)$ in (2.2) independently of its observation $y_{k}$.

- A herd of agents takes place at time $\bar{k}$, if the actions of all agents after time $\bar{k}$ are identical, i.e., $a_{k}=a_{\bar{k}}$ for all time $k>\bar{k}$.

- An information cascade occurs at time $\bar{k}$, if the public beliefs of all agents after time $\bar{k}$ are identical, i.e. $\pi_{k}=\pi_{\bar{k}}$ for all $k>\bar{k}$.

Note that if an information cascade occurs, then since the public belief freezes, social learning ceases. Also, from the above definitions, it is clear that an information cascade implies a herd of agents, but the reverse is not true; see $\$ 2.3$ for an example.

The following result, which is well known in the economics literature [31, 50], states that if agents follow the above social learning protocol, then after some finite time $\bar{k}$, an information cascade occurs 3 . The proof follows via an elementary application of the martingale convergence theorem.

Theorem 2.2.1 ([31]). The social learning protocol described in $\$ 2.2 .1$ leads to an information cascade in finite time with probability 1. That is, there exists a finite time $\bar{k}$ after which social learning ceases, i.e., public belief $\pi_{k+1}=\pi_{k}$, $k \geq \bar{k}$, and all agents choose the same action, i.e., $a_{k+1}=a_{k}, k \geq \bar{k}$.

\footnotetext{
${ }^{3} \mathrm{~A}$ nice analogy is provided in [50]. If I see someone walking down the street with an umbrella, I assume (based on rationality) that he has checked the weather forecast and is carrying an umbrella since it might rain. Therefore, I also take an umbrella. Now, there are two people walking down the street carrying umbrellas. A third person sees two people with umbrellas and, based on the same inference logic, also takes an umbrella. Even though each individual is rational, such herding behavior might be irrational since the first person who took the umbrella may not have checked the weather forecast.

Another example is that of patrons who decide to choose a restaurant. Despite their menu preferences, each patron chooses the restaurant with the most customers. Therefore, all patrons eventually herd to one restaurant. The paper [245] quotes the following anecdote on user influence in a social network which can be interpreted as herding: “... when a popular blogger left his blogging site for a two-week vacation, the site's visitor tally fell, and content produced by three invited substitute bloggers could not stem the decline."
} 
Instead of reproducing the proof, let us give some insight as to why Theorem 2.2.1 holds. It can be shown using martingale methods that, at some finite time $k=\bar{k}$, the agent's probability $\mathbb{P}\left(a_{k} \mid y_{k}, \pi_{k-1}\right)$ becomes independent of the private observation $y_{k}$. Then, clearly from (2.4), $\mathbb{P}\left(a_{k}=a \mid x_{k}=i, \pi_{k-1}\right)=$ $\mathbb{P}\left(a_{k}=a \mid \pi\right)$. Substituting this into the social learning filter $(2.3)$, we see that $\pi_{k}=\pi_{k-1}$. Thus, after some finite time $\bar{k}$, the social learning filter hits a fixed point and social learning stops. As a result, all subsequent agents $k>\bar{k}$ completely disregard their private observations and take the same action $a_{\bar{k}}$, thereby forming an information cascade (and therefore a herd).

\subsubsection{Constrained Interactive Sensing: Individual Privacy vs Group Reputation}

The above social learning protocol can be interpreted as follows. Agents seek to estimate an underlying state of nature; however, they reveal their actions by maximizing their privacy according to the optimization (2.2). This leads to an information cascade and social learning stops. In other words, agents are interested in optimizing their own costs (such as maximizing privacy) and ignore the information benefits their action provides to others.

\section{Partially Observed Markov Decision Process Formulation}

We now describe an optimized social learning procedure that delays herding 4 . This approach is motivated by the following question: How can agents assist social learning by choosing their actions to trade off individual privacy (local costs) with optimizing the reputation 5 of the entire social group? (See [50] for an excellent discussion.)

Suppose agents seek to maximize the reputation of their social group by minimizing the following social welfare cost involving all agents in the social group

\footnotetext{
${ }^{4}$ In the restaurant problem, an obvious approach to prevent herding is as follows. If a restaurant knew that patrons choose the restaurant with the most customers, then the restaurant could deliberately pay actors to sit in the restaurant so that it appears popular, thereby attracting customers. The methodology in this section, where herding is delayed by benevolent agents, is a different approach.

${ }^{5}$ The papers [197, 110] contain lucid descriptions of quantitative models for trust, reputation and privacy.
} 
(compared to the myopic objective (2.2) used in standard social learning):

$$
J_{\mu}\left(\pi_{0}\right)=\mathbf{E}_{\pi_{0}}^{\mu}\left\{\sum_{k=1}^{\infty} \rho^{k-1} c_{a\left(\pi_{k-1}, y_{k}, \mu\left(\pi_{k-1}\right)\right)}^{\prime} \eta_{k}\right\} .
$$

In (2.7), $a(\pi, y, \mu(\pi)))$ denotes the decision rule that agents use to choose their actions as will be explained below. Also, $\rho \in[0,1)$ is an economic discount factor, and $\pi_{0}$ denotes the initial probability (prior) of the state $x$. Finally, $\mathbf{P}_{\pi_{0}}^{\mu}$ and $\mathbf{E}_{\pi_{0}}^{\mu}$ denote the probability measure and expectation of the evolution of the observations and underlying state, respectively, which are strategy dependent.

The key attribute of (2.7) is that each agent $k$ chooses its action according to the privacy constrained rule

$$
a_{k}=a\left(\pi_{k-1}, y_{k}, \mu\left(\pi_{k-1}\right)\right)
$$

Here, the policy

$$
\mu: \pi_{k-1} \rightarrow\{1,2 \ldots, L\}
$$

maps the available public belief to the set of $L$ privacy values. The higher the privacy value, the less the agent reveals through its action. This is in contrast to standard social learning (2.2) in which the action chosen is $a(\pi, y)$, namely, a myopic function of the private observation and public belief.

The above formulation can be interpreted as follows: Individual agents seek to maximize their privacy according to social learning (2.8), but also seek to maximize the reputation of their entire social group (2.7).

Determining the policy $\mu^{*}$ that minimizes $(2.7)$, and thereby maximizes the social group reputation, is equivalent to solving a stochastic control problem that is called a partially observed Markov decision process (POMDP) problem [48, 153]. A POMDP comprises of a noisy observed Markov chain and the dynamics of the posterior distribution (belief state) is controlled by a policy ( $\mu$ in our case).

\section{Structure of Privacy Constrained Sensing Policy}

In general, POMDPs are computationally intractable to solve [209] and, therefore, one cannot say anything useful about the structure 6 of the optimal policy

\footnotetext{
${ }^{6}$ Characterizing the structure of the optimal policy of a POMDP is a difficult problem. We refer the reader to [186, 185, 218, 157, 153] for sufficient conditions (based on supermodularity that yield a monotone optimal policy for a POMDP in terms of the monotone likelihood ratio stochastic order.
} 
$\mu^{*}$. However, useful insight can be obtained by considering the following extreme case of the above problem. Suppose there are two privacy values and each agent $k$ chooses action

$$
a_{k}= \begin{cases}y_{k}, & \text { if } \mu\left(\pi_{k}\right)=1 \text { (no privacy), } \\ \operatorname{argmin}_{a} c_{a}^{\prime} \pi_{k-1}, & \text { if } \mu\left(\pi_{k}\right)=2 \text { (full privacy) }\end{cases}
$$

That is, an agent either reveals its raw observation (no privacy) or chooses its action by completely neglecting its observation (full privacy). Once an agent chooses the full privacy option, then all subsequent agents choose exactly the same option and therefore herd-this follows since each agent's action reveals nothing about the underlying state of nature. Therefore, for this extreme example, determining the optimal policy $\mu^{*}(\pi)$ is equivalent to solving a stopping time problem: Determine the earliest time for agents to herd (maintain full privacy) subject to maximizing the social group reputation.

For such a quickest herding stopping time problem, one can say a lot about the structure of $\mu^{*}(\pi)$. Suppose the sensing system wishes to determine if the state of nature is a specific target state (say state 1). Then, [153] shows that, under reasonable conditions on the observation distribution and supermodular conditions on the costs ([193] discusses supermodularity of influence in social networks), the dynamic programming recursion has a supermodular structure 7 (see also [154, 155, 157, 186, 218] for related results). This implies that the optimal policy $\mu^{*}$ has the following structure: There exists a threshold curve that partitions the belief space such that, when the belief state is on one side of the curve, it is optimal for agents to reveal full observations; if the belief state is on the other side of the curve, then it is optimal to herd. Moreover, the target state 1 belongs to the region in which it is optimal to herd 8 . This threshold structure of the optimal policy means that if individuals deploy the simple heuristic of

\section{"Choose increased privacy when belief is close to target state,"}

then the group behavior is sophisticated-herding is delayed and accurate estimates of the state of nature can be obtained.

\footnotetext{
${ }^{7}$ The seminal book on supermodularity is [243], also see [10] for a lucid tutorial presentation.

${ }^{8}$ In standard POMDPs where agents do not perform social learning, it is well known [185] that the set of beliefs for which it is optimal to stop is convex. Such convexity of the herding set does not hold in the current problem. But it is shown in [153] that the set of beliefs for which it is optimal to herd constitute a connected set and so does the set of beliefs for which it is optimal to reveal full observations.
} 


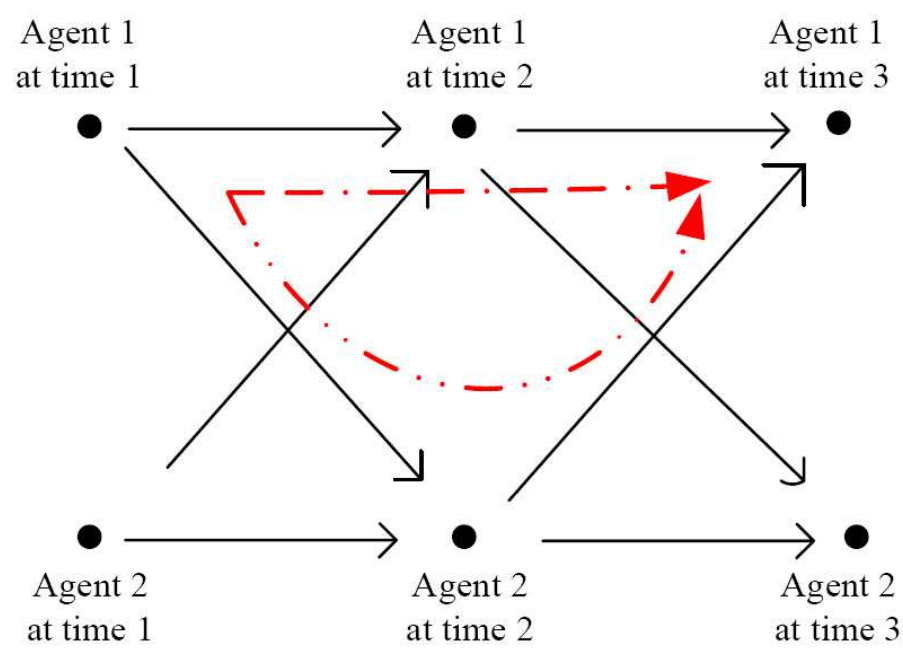

Figure 2.1: Example of the information flow in a social network with two agents over three event epochs. The arrows represent exchange of information.

\subsection{Data Incest in Online Reputation Systems}

This section generalizes the previous section by considering social learning in a social network. How can multiple social sensors interacting over a social network estimate an underlying state of nature? The state could be the position coordinates of an event [222] or the quality of a social parameter such as quality of a restaurant or political party.

The motivation for this section can be understood in terms of the following sensing example. Consider the following interactions in a multi-agent social network where agents seek to estimate an underlying state of nature. Each agent visits a restaurant based on reviews on an online reputation website. The agent then obtains a private measurement of the state (e.g., the quality of food in a restaurant) in noise. After that, he reviews the restaurant on the same online reputation website. The information exchange in the social network is modeled by a directed graph. As mentioned in Chapter 1, data incest [160] arises due to the loops in the information exchange graph. This is illustrated in Figure 2.1: Agents 1 and 2 exchange beliefs (or actions). The fact that there exist two distinct paths between Agent 1 at time 1 and Agent 1 at time 3 (depicted in red in Figure 2.1) implies that the information of Agent 1 at time 1 is double counted, thereby leading to a data incest event. 
How can data incest be removed so that agents obtain a fair (unbiased) estimate of the underlying state? The methodology of this section can be interpreted in terms of the recent Time article [247], which provides interesting rules for online reputation systems. These include: (i) review the reviewers, and (ii) censor fake (malicious) reviewers. The data incest removal algorithm proposed in this section can be viewed as "reviewing the reviews" of other agents to see if they are associated with data incest or not.

The rest of this section is organized as follows:

1. Section 2.3.1 describes the social learning model that is used to mimic the behavior of agents in online reputation systems. The information exchange between agents in the social network is formulated on a family of timedependent directed acyclic graphs.

2. In $\$ 2.3 .2$, a fair reputation protocol is presented and the criterion for achieving a fair rating is defined.

3. Section 2.3.3 presents an incest removal algorithm so that the online reputation system achieves a fair rating. A necessary and sufficient condition is given on the graph that represents exchange of information between agents so as to achieve fair ratings.

Related works Collaborative recommendation systems are reviewed and studied in [4, 147]. In [136], a model of Bayesian social learning is considered in which agents receive private information about the state of nature and observe actions of their neighbors in a tree-based network. Another type of misinformation caused by influential agents (agents who heavily affect actions of other agents in social networks) is investigated in [2]. Misinformation in the context of this section is motivated by sensor networks where the term "data incest" is used [39]. Data incest also arises in belief propagation (BP) algorithms [198, 213], which are used in computer vision and error-correcting coding theory. BP algorithms require passing local messages over the graph (Bayesian network) at each iteration. For graphical models with loops, BP algorithms are only approximate due to the overcounting of local messages [258], which is similar to data incest in social learning. With the algorithms presented in this section, data incest can be mitigated from Bayesian social learning over non-tree graphs that satisfy a topological constraint. The closest work to the current section is [160]. However, in [160], data incest is considered in a network where agents exchange their private belief states - that is, 
no social learning is considered. Simpler versions of this information exchange process and estimation were investigated in [19, 36, 97].

\subsubsection{Information Exchange Graph}

Consider an online reputation system comprised of social sensors $\{1,2, \ldots, S\}$ that aim to estimate an underlying state of nature (a random variable). Let $x \in$ $\mathbb{X}=\{1,2, \ldots, X\}$ represent the state of nature (such as the quality of a hotel) with known prior distribution $\pi_{0}$. Let $k=1,2,3, \ldots$ represent epochs at which events occur. These events involve taking observations, evaluating beliefs and choosing actions as described below. The index $k$ marks the historical order of events, and not necessarily absolute time. However, for simplicity, we refer to $k$ as "time".

To model the information exchange in the social network, we will use a family of directed acyclic graphs. It is convenient also to reduce the coordinates of time $k$ and agent $s$ to a single integer index $n$ as follows:

$$
n \stackrel{\text { defn }}{=} s+S(k-1), \quad s \in\{1, \ldots, S\}, k=1,2,3, \ldots
$$

We refer to $n$ as a "node" of a time-dependent information flow graph $G_{n}$ that we define below.

\section{Some Graph Theoretic Definitions}

Let

$$
G_{n}=\left(V_{n}, E_{n}\right), \quad n=1,2, \ldots
$$

denote a sequence of time-dependent graphs of information flow in the social network until and including time $k$ where $n=s+S(k-1)$. Each vertex in $V_{n}$ represents an agent $s^{\prime}$ in the social network at time $k^{\prime}$, and each edge $\left(n^{\prime}, n^{\prime \prime}\right)$ in $E_{n} \subseteq V_{n} \times V_{n}$ shows that the information (action) of node $n^{\prime}$ (agent $s^{\prime}$ at time $k^{\prime}$ ) reaches node $n^{\prime \prime}$ (agent $s^{\prime \prime}$ at time $k^{\prime \prime}$ ). It is clear that the communication graph $G_{n}$ is a sub-graph of $G_{n+1}$. This means that the diffusion of actions can be modelled via a family of time-dependent directed acyclic graphs?

The algorithms below will involve specific columns of the adjacency matrix and transitive closure matrix of the graph $G_{n}$. The adjacency matrix $A_{n}$ of $G_{n}$ is

\footnotetext{
${ }^{9}$ Directed acyclic graphs are directed graph with no directed cycles.
} 
an $n \times n$ matrix with elements $A_{n}(i, j)$ given by

$$
A_{n}(i, j)=\left\{\begin{array}{ll}
1, & \text { if }\left(v_{j}, v_{i}\right) \in E \\
0, & \text { otherwise }
\end{array}, \quad A_{n}(i, i)=0\right.
$$

The transitive closure matrix $T_{n}$ is the $n \times n$ matrix

$$
T_{n}=\operatorname{sgn}\left(\left(\mathbf{I}_{n}-A_{n}\right)^{-1}\right)
$$

where for any matrix $M$, the matrix $\operatorname{sgn}(M)$ has elements

$$
\operatorname{sgn}(M)(i, j)= \begin{cases}0, & \text { if } M(i, j)=0 \\ 1, & \text { if } M(i, j) \neq 0\end{cases}
$$

Note that $A_{n}(i, j)=1$ if there is a single-hop path between nodes $i$ and $j$. In comparison, $T_{n}(i, j)=1$ if there exists a (possibly multi-hop) path between node $i$ and $j$.

The information reaching node $n$ depends on the information flow graph $G_{n}$. The following two sets will be used to specify the incest removal algorithms below:

$$
\begin{aligned}
\mathcal{H}_{n} & =\left\{m: A_{n}(m, n)=1\right\} \\
\mathcal{F}_{n} & =\left\{m: T_{n}(m, n)=1\right\}
\end{aligned}
$$

Here, $\mathcal{H}_{n}$ denotes the set of previous nodes $m$ that communicate with node $n$ in a single-hop. In comparison, $\mathcal{F}_{n}$ denotes the set of previous nodes $m$ whose information eventually arrive at node $n$. Thus, $\mathcal{F}_{n}$ contains all possible multi-hop connections by which information from a node $m$ eventually reaches node $n$.

Note that classical social learning of Sec.2.2.1 is a special case with adjacency matrix $A_{n}(i, j)=1$ for $j=i+1$ and $A_{n}(i, j)=0$ elsewhere.

Example 2.1. To illustrate the above notation consider a social network consisting of $S=2$ agents with the following information flow graph for three time points $k=1,2,3$. Figure 2.2 depicts the nodes $n=1,2, \ldots, 6$, where $n=s+2(k-1)$.

Note that, in this example, each node remembers all its previous actions as is apparent from Figure 2.1. The information flow is characterized by the family of 


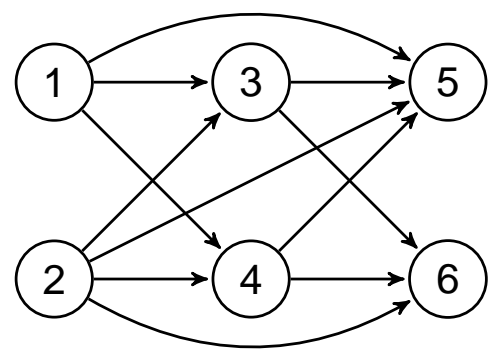

Figure 2.2: Example of an information flow network with two agents $(S=2)$, namely, $s \in\{1,2\}$ and time instants $k=1,2,3$. Circles represent the nodes indexed by $n=s+S(k-1)$ in the social network, and each edge depicts a communication link between two nodes.

directed acyclic graphs $\left\{G_{1}, G_{2}, G_{3}, G_{4}, G_{5}, G_{6}\right\}$ with adjacency matrices

$$
\begin{aligned}
& A_{1}=[0], A_{2}=\left[\begin{array}{ll}
0 & 0 \\
0 & 0
\end{array}\right], A_{3}=\left[\begin{array}{lll}
0 & 0 & 1 \\
0 & 0 & 1 \\
0 & 0 & 0
\end{array}\right], \\
& A_{4}=\left[\begin{array}{llll}
0 & 0 & 1 & 1 \\
0 & 0 & 1 & 1 \\
0 & 0 & 0 & 0 \\
0 & 0 & 0 & 0
\end{array}\right], A_{5}=\left[\begin{array}{lllll}
0 & 0 & 1 & 1 & 1 \\
0 & 0 & 1 & 1 & 1 \\
0 & 0 & 0 & 0 & 1 \\
0 & 0 & 0 & 0 & 1 \\
0 & 0 & 0 & 0 & 0
\end{array}\right] .
\end{aligned}
$$

Since nodes 1 and 2 do not communicate, clearly $A_{1}$ and $A_{2}$ are zero matrices. Nodes 1 and 3 communicate as do nodes 2 and 3, hence, $A_{3}$ has two ones, etc. Finally from (2.15) and (2.16),

$$
\mathcal{H}_{5}=\{1,2,3,4\}, \quad \mathcal{F}_{5}=\{1,2,3,4\}
$$

where $\mathcal{H}_{5}$ denotes all single-hop links to node 5 , whereas $\mathcal{F}_{5}$ denotes all multi-hop links to node 5.

Note that $A_{n}$ is always the upper left $n \times n$ submatrix of $A_{n+1}$. Also due to causality with respect to the time index $k$, the adjacency matrices are always upper triangular.

\subsubsection{Fair Online Reputation System}

The aim is to provide each node $n$ with an unbiased estimate

$$
\pi_{n-}^{0}(i)=P\left(x=i \mid\left\{a_{m}, m \in \mathcal{F}_{n}\right\}\right)
$$


subject to the following social influence constraint: There exists a fusion algorithm $\mathcal{A}$ such that

$$
\pi_{n-}^{0}=\mathcal{A}\left(\pi_{m}, m \in \mathcal{H}_{n}\right) .
$$

We call $\pi_{n-}^{0}$ in (2.17) the true or fair online rating available to node $n$. Recall that $\mathcal{F}_{n}$, defined in (2.16), denotes all information (multi-hop links) available to node $n$. By definition, $\pi_{n-}^{0}$ is incest free since it is the desired conditional probability that we want.

The procedure summarized in Protocol 1 aims to evaluate a fair reputation that uses social learning over a social network by eliminating incest.

If algorithm $\mathcal{A}$ is designed so that $\pi_{n-}(i)$ satisfies (2.17), then the computation (2.20) and Step (v) yield

$$
\begin{aligned}
& \eta_{n}(i)=\mathbb{P}\left(x=i \mid\left\{a_{m}, m \in \mathcal{F}_{n}\right\}, y_{n}\right), \\
& \pi_{n}(i)=\mathbb{P}\left(x=i \mid\left\{a_{m}, m \in \mathcal{F}_{n}\right\}, a_{n}\right), \quad i \in \mathbb{X}
\end{aligned}
$$

which are, respectively, the correct private belief for node $n$ and the correct afteraction public belief.

\section{Discussion of Protocol 1}

(i) Data Incest: It is important to note that without careful design of algorithm $\mathcal{A}$, due to loops in the dependencies of actions on previous actions, the public rating $\pi_{n-}$ computed using (2.19) can be substantially different from the fair online rating $\pi_{n-}^{0}$ of (2.17). As a result, $\eta_{n}$ computed via (2.20) will not be the correct private belief and incest will propagate in the network. In other words, $\eta_{n}, \pi_{n-}$, and $\pi_{n}$ are defined purely in terms of their computational expressions in Protocol 1-at this stage, they are not necessarily the desired conditional probabilities unless algorithm $\mathcal{A}$ is designed to remove incest.

Note that, instead of (2.19), node $n$ could naively (and incorrectly) assume that the public beliefs $\pi_{m}, m \in \mathcal{H}_{n}$, that it received are independent. It would then fuse these public beliefs as

$$
\pi_{n-}=\frac{\prod_{m \in \mathcal{H}_{n}} \pi_{m}}{\mathbf{1}_{X}^{\prime} \prod_{m \in \mathcal{H}_{n}} \pi_{m}} .
$$

This, of course, would result in data incest.

(ii) How much does an individual remember?: The above protocol has the flexibility of modelling cases where either each node remembers some (or all) 
Protocol 1 Incest Removal for Social Learning in Online Reputation Systems

(i) Information from Social Network:

1. Recommendation from friends: Node $n$ receives past actions $\left\{a_{m}, m \in \mathcal{H}_{n}\right\}$ from previous nodes $m \in \mathcal{H}_{n}$ in the social network. $\mathcal{H}_{n}$ is defined in (2.15).

2. Automated Recommender System: For these past actions $\left\{a_{m}, m \in \mathcal{H}_{n}\right\}$, the network administrator has already computed the public beliefs $\left(\pi_{m}, m \in\right.$ $\mathcal{H}_{n}$ ) using Step (v) below.

The automated recommender system fuses public beliefs $\left(\pi_{m}, m \in \mathcal{H}_{n}\right)$, into the single recommendation belief $\pi_{n-}$ as

$$
\pi_{n-}=\mathcal{A}\left(\pi_{m}, m \in \mathcal{H}_{n}\right) .
$$

The fusion algorithm $\mathcal{A}$ will be designed below.

(ii) Observation: Node $n$ records private observation $y_{n}$ from distribution $B_{i y}=$ $\mathbb{P}(y \mid x=i), i \in \mathbb{X}$.

(iii) Private Belief: Node $n$ then uses $y_{n}$ and public belief $\pi_{n-}$ to update its private belief via Bayes formula as

$$
\eta_{n}=\frac{B_{y_{n}} \pi_{n-}}{\mathbf{1}_{X}^{\prime} B_{y} \pi_{n-}}
$$

(iv) Myopic Action: Node $n$ takes action

$$
a_{n}=\underset{a}{\operatorname{argmin}} c_{a}^{\prime} \eta_{n}
$$

and inputs its action to the online reputation system.

(v) Public Belief Update by Network Administrator: Based on action $a_{n}$, the network administrator (automated algorithm) computes the public belief $\pi_{n}$ using the social learning filter (2.3) with $P=I$. 
of its past actions or none of its past actions. This facilitates modelling cases in which people forget most of the past except for specific highlights.

(iii) Automated Recommender System: Steps (i) and (v) of Protocol 1 can be combined into an automated recommender system that maps previous actions of agents to a single recommendation (rating) $\pi_{n-}$ of (2.19). This recommender system can operate completely opaquely to the actual user (node $n$ ). Node $n$ simply uses the automated rating $\pi_{n-}$ as the current best available rating from the reputation system.

(iv) Social Influence. Informational Message vs Social Message: In Proto$\operatorname{col} 1$ it is important that each individual $n$ deploys Algorithm $\mathcal{A}$ to fuse the beliefs $\left\{\pi_{m}, m \in \mathcal{H}_{n}\right\}$; otherwise, incest can propagate. Here, $\mathcal{H}_{n}$ can be viewed as the "social message", i.e., personal friends of node $n$ since they directly communicate to node $n$, while the associated beliefs can be viewed as the "informational message". The social message from personal friends exerts a large social influence-it provides significant incentive (peer pressure) for individual $n$ to comply with Protocol 11 and thereby prevent incest. Indeed, a remarkable recent study described in [35] shows that social messages (votes) from known friends has significantly more influence on an individual than the information in the messages themselves. This study includes comparison of information messages and social messages on Facebook and their direct effect on voting behavior. To quote [35],

"The effect of social transmission on real-world voting was greater than the direct effect of the messages themselves..."

(v) Agent Reputation: The cost function minimization in Step (iv) can be interpreted in terms of the reputation of agents in online reputation systems. If an agent continues to write bad reviews for high quality restaurants on Yelp, his reputation declines among the users. Consequently, other people ignore reviews of that (low-reputation) agent in evaluating their opinion about the social unit under study (restaurant). Therefore, agents minimize the penalty of writing inaccurate reviews (or equivalently increase their reputations) by choosing proper actions.

(vi) Think and act: Steps (ii), (iii), (iv), and (v) of Protocol 1 constitute standard social learning as described in $\$ 2.2 .1$ The key difference with standard social learning is Step (i) that is performed by the network administrator. Agents receive public beliefs from the social network with arbitrary random delays. These delays reflect the time an agent takes between reading the publicly available reputation and making its decision. It is typical behavior of people to read published ratings multiple times and then think for an arbitrary amount of time before acting. 


\subsubsection{Incest Removal Algorithm in Online Reputation System}

Below we design algorithm $\mathcal{A}$ in Protocol 1 so that it yields the fair public rating $\pi_{n-}^{0}$ of (2.17).

\section{Fair Rating Algorithm}

It is convenient to work with the logarithm of the un-normalized belief 10 . Accordingly, define

$$
l_{n}(i) \propto \log \pi_{n}(i), \quad l_{n-}(i) \propto \log \pi_{n-}(i), \quad i \in \mathbb{X}
$$

The following theorem shows that the logarithm of the fair rating $\pi_{n-}^{0}$ defined in (2.17) can be obtained as linear weighted combination of the logarithms of previous public beliefs.

Theorem 2.3.1 (Fair Rating Algorithm). Consider the online reputation system running Protocol 1 Suppose the following algorithm $\mathcal{A}\left(l_{m}, m \in \mathcal{H}_{n}\right)$ is implemented in (2.19) of Protocol 1 by the network administrator:

$$
l_{n-}(i)=w_{n}^{\prime} l_{1: n-1}(i), \text { where } w_{n}=T_{n-1}^{-1} t_{n} .
$$

Then, $l_{n-}(i) \propto \log \pi_{n-}^{0}(i)$. That is, algorithm $\mathcal{A}$ computes the fair rating $\log \pi_{n-}^{0}(i)$ defined in (2.17).

In (2.25), $w_{n}$ is an $n-1$ dimensional weight vector. Recall that $t_{n}$ denotes the first $n-1$ elements of the nth column of transitive closure matrix $T_{n}$.

Theorem 2.3.1 says that the fair rating $\pi_{n-}^{0}$ can be expressed as a linear function of the action log-likelihoods in terms of the transitive closure matrix $T_{n}$ of the information flow graph $G_{n}$. This is intuitive since $\pi_{n-}^{0}$ can be viewed as the sum of information collected by the nodes such that there are paths between all these nodes and $n$.

\footnotetext{
${ }^{10}$ The un-normalized belief proportional to $\pi_{n}(i)$ is the numerator of the social learning filter (2.3). The corresponding un-normalized fair rating corresponding to $\pi_{n-}^{0}(i)$ is the joint distribution $\mathbb{P}\left(x=i,\left\{a_{m}, m \in \mathcal{F}_{n}\right\}\right)$. By taking logarithm of the un-normalized belief, Bayes formula merely becomes the sum of the log likelihood and log prior. This allows us to devise a data incest removal algorithm based on linear combinations of the log beliefs.
} 


\section{Achievability of Fair Rating by Protocol 1}

We are not quite done!

- On the one hand, algorithm $\mathcal{A}$ at node $n$ specified by (2.19) has access only to beliefs $l_{m}, m \in \mathcal{H}_{n}$-equivalently, it has access only to beliefs from previous nodes specified by $A_{n}(:, n)$, which denotes the last column of the adjacency matrix $A_{n}$.

- On the other hand, to provide incest free estimates, algorithm $\mathcal{A}$ specified in (2.25) requires all previous beliefs $l_{1: n-1}(i)$ that are specified by the nonzero elements of the vector $w_{n}$.

The only way to reconcile the above points is to ensure that $A_{n}(j, n)=0$ implies $w_{n}(j)=0$ for $j=1, \ldots, n-1$. This condition means that the single hop past estimates $l_{m}, m \in \mathcal{H}_{n}$, available at node $n$ according to (2.19) in Protocol11 provide all the information that is required to compute $w_{n}^{\prime} l_{1: n-1}$ in (2.25). This is essentially a condition on the information flow graph $G_{n}$. We formalize this condition in the following theorem.

Theorem 2.3.2 (Achievability of Fair Rating). Consider the fair rating algorithm specified by (2.25). For Protocol 1 with available information $\left(\pi_{m}, m \in \mathcal{H}_{n}\right)$, to achieve the estimates $l_{n-}$ of algorithm (2.25), a necessary and sufficient condition on the information flow graph $G_{n}$ is

$$
A_{n}(j, n)=0 \Longrightarrow w_{n}(j)=0
$$

Therefore, for Protocol $\square$ to generate incest free estimates for nodes $n=1,2, \ldots$, condition (2.26) needs to hold for each $n$. (Recall $w_{n}$ is specified in (2.25).)

Note that the constraint (2.26) is purely in terms of the adjacency matrix $A_{n}$ since the transitive closure matrix (2.13) is a function of the adjacency matrix. Therefore, Algorithm (2.25), together with condition (2.26), ensures that incest free estimates are generated by Protocol 1 .

Example 2.2. Let us continue with Example 2.1 where we already specified the adjacency matrices of the graphs $G_{1}, G_{2}, G_{3}, G_{4}$, and $G_{5}$. Using (2.13), the 
transitive closure matrices $T_{n}$ obtained from the adjacency matrices are given by:

$$
\begin{aligned}
& T_{1}=[1], T_{2}=\left[\begin{array}{ll}
1 & 0 \\
0 & 1
\end{array}\right], T_{3}=\left[\begin{array}{lll}
1 & 0 & 1 \\
0 & 1 & 1 \\
0 & 0 & 1
\end{array}\right], \\
& T_{4}=\left[\begin{array}{llll}
1 & 0 & 1 & 1 \\
0 & 1 & 1 & 1 \\
0 & 0 & 1 & 0 \\
0 & 0 & 0 & 1
\end{array}\right], T_{5}=\left[\begin{array}{lllll}
1 & 0 & 1 & 1 & 1 \\
0 & 1 & 1 & 1 & 1 \\
0 & 0 & 1 & 0 & 1 \\
0 & 0 & 0 & 1 & 1 \\
0 & 0 & 0 & 0 & 1
\end{array}\right] .
\end{aligned}
$$

Note that $T_{n}(i, j)$ is non-zero only for $i \geq j$ due to causality-information sent by an agent can only arrive at another agent at a later time instant. The weight vectors are then obtained from (2.25) as

$$
\begin{aligned}
& w_{2}=[0], \\
& w_{3}=\left[\begin{array}{ll}
1 & 1
\end{array}\right]^{\prime}, \\
& w_{4}=\left[\begin{array}{lll}
1 & 1 & 0
\end{array}\right]^{\prime}, \\
& w_{5}=\left[\begin{array}{llll}
-1 & -1 & 1 & 1
\end{array}\right]^{\prime} .
\end{aligned}
$$

Let us examine these weight vectors. $w_{2}$ means that node 2 does not use the estimate from node 1. This formula is consistent with the constrained information flow because estimate from node 1 is not available to node 2; see Figure 2.2 $w_{3}$ means that node 3 uses estimates from node 1 and $2 . w_{4}$ means that node 4 uses estimates only from node 1 and node 2 since the estimate from node 3 is not available at node 4. As shown in Figure 2.2. the mis-information propagation occurs at node 5. The vector $w_{5}$ says that node 5 adds estimates from nodes 3 and 4 and removes estimates from nodes 1 and 2 to avoid double counting of these estimates that are already integrated into estimates from node 3 and 4 . Indeed, using the algorithm (2.25), incest is completely prevented in this example.

Let us now illustrate an example in which exact incest removal is impossible. Consider the information flow graph of Figure 2.2] but with the edge between nodes 2 and 5 deleted. Then, $A_{5}(2,5)=0$, while $w_{5}(2) \neq 0$; therefore, the condition (2.26) does not hold. Hence, exact incest removal is not possible for this case.

\subsubsection{Summary}

In this section, we have outlined a controlled sensing problem over a social network in which the administrator controls (removes) data incest, and thereby main- 
tains an unbiased (fair) online reputation system. The state of nature could be geographical coordinates of an event (in a target localization problem) or quality of a social unit (in an online reputation system). As discussed above, data incest arises due to the recursive nature of Bayesian estimation and non-determinism in the timing of the sensing by individuals. Details of proofs, extensions and further numerical studies are presented in [113, 160].

How useful are Bayesian social learning models? Humans often make monotone decisions - the more favorable the private observation, the higher the recommendation. In addition, humans typically convert numerical attributes to ordinal scales before making a decision. For example, it does not make a difference if the cost of a meal at a restaurant is $\$ 200$ or $\$ 205$; an individual would classify this cost as "high". Also credit rating agencies use ordinal symbols such as AAA, AA, A. It is shown in [154] that if the cost $c(x, a)$ satisfies a single crossing condition 11 and the observation likelihoods satisfy a totally positive condition, then the recommendation $a_{n}$ made by node $n$ is monotone increasing in its observation $y_{n}$ and ordinal. In this sense, even if an agent does not exactly follow a Bayesian social learning model, its monotone ordinal behavior implies that such a model is a useful idealization.

\subsection{Interactive Sensing for Quickest Change Detec- tion}

In this section, we consider interacting social sensors in the context of detecting a change in the underlying state of nature. Suppose a multi-agent system performs social learning and makes local decisions as described in $\$ 2.2$. Given the public beliefs from the social learning protocol, how can quickest change detection be achieved? In other words, how can a global decision maker use the local decisions from individual agents to decide when a change has occurred? It is shown below that making a global decision (change or no change) based on local decisions of individual agents has an unusual structure resulting in a non-convex stopping set.

A typical application of such social sensors arises in the measurement of the adoption of a new product using a micro-blogging platform like Twitter. The adoption of the technology diffuses through the market but its effects can only be observed through the tweets of select individuals of the population. These

\footnotetext{
${ }^{11}$ The single crossing condition can be viewed as an ordinal generalization of supermodularity [10]. Supermodularity is a sufficient condition for a single crossing condition to hold.
} 
selected individuals act as sensors for estimating the diffusion. They interact and learn from the decisions (tweeted sentiments) of other members and, therefore, perform social learning. Suppose the state of nature suddenly changes due to a sudden market shock or presence of a new competitor. The goal for a market analyst or product manufacturer is to detect this change as quickly as possible by minimizing a cost function that involves the sum of the false alarm and decision delay.

Related works The papers [183, 184] model diffusion in networks over a random graph with arbitrary degree distribution. The resulting diffusion is approximated using deterministic dynamics via a mean-field approach [27]. In the seminal paper [75], a sensing system for complex social systems is presented with data collected from cell phones. This data is used to recognize social patterns, identify socially significant locations, and infer relationships. In [222], people using a microblogging service such as Twitter are considered as sensors. A particle filtering algorithm is then used to estimate the centre of earthquakes and trajectories of typhoons. As pointed out in [222], an important characteristic of microblogging services such as Twitter is that they provide real-time sensing-Twitter users tweet several times a day, whereas standard blog users update information once every several days.

Apart from the above applications in real-time sensing, change detection in social learning also arises in mathematical finance models. For example, in agent based models for the microstructure of asset prices in high frequency trading in financial systems [21], the state denotes the underlying asset value that changes at a random time $\tau^{0}$. Agents observe local individual decisions of previous agents via an order book, combine these observed decisions with their noisy private signals about the asset, selfishly optimize their expected local utilities, and then make their own individual decisions (whether to buy, sell or do nothing). The market evolves through the orders of trading agents. Given this order book information, the goal of the market maker (global decision maker) is to achieve quickest change point detection of when a shock occurs to the value of the asset [156].

\subsubsection{Classical Quickest Detection}

The classical Bayesian quickest time detection problem [214] is as follows: An underlying discrete-time state process $x$ jump-changes at a geometrically distributed random time $\tau^{0}$. Consider a sequence of discrete time random measure- 
ments $\left\{y_{k}, k \geq 1\right\}$ such that, conditioned on the event $\left\{\tau^{0}=t\right\}, y_{k}, k \leq t$, are independent and identically distributed (i.i.d.) random variables with distribution $B_{1}$ and $y_{k}, k>t$, are i.i.d. random variables with distribution $B_{2}$. The quickest detection problem involves detecting the change time $\tau^{0}$ with minimal cost. That is, at each time $k=1,2, \ldots$, a decision $u_{k} \in\{1$ (stop and announce change), 2 (continue) $\}$ needs to be made to optimize a tradeoff between false alarm frequency and linear delay penalty.

To formalize this setup, let

$$
P=\left[\begin{array}{cc}
1 & 0 \\
1-P_{22} & P_{22}
\end{array}\right]
$$

denote the transition matrix of a two state Markov chain $x$ in which state 1 is absorbing. Then, it is easily seen that the geometrically distributed change time $\tau^{0}$ is equivalent to the time at which the Markov chain enters state 1 . That is,

$$
\tau^{0}=\min \left\{k: x_{k}=1\right\}, \text { and } \mathbf{E}\left\{\tau^{0}\right\}=1 /\left(1-P_{22}\right) .
$$

Let $\tau$ be the time at which the decision $u_{k}=1$ (announce change) is made. The goal of quickest time detection is to minimize the Kolmogorov-Shiryaev criterion for detection of a disorder [231]:

$$
J_{\mu}\left(\pi_{0}\right)=d \mathbf{E}_{\pi_{0}}^{\mu}\left\{\left(\tau-\tau^{0}\right)^{+}\right\}+f \mathbf{P}_{\pi_{0}}^{\mu}\left(\tau<\tau^{0}\right) .
$$

Here, $x^{+}=x$ if $x \geq 0$, and 0 otherwise. The non-negative constants $d$ and $f$ denote the delay and false alarm penalties, respectively. Therefore, waiting too long to announce a change incurs a delay penalty $d$ at each time instant after the system has changed, while declaring a change before it happens incurs a false alarm penalty $f$. In (2.28), $\mu$ denotes the strategy of the decision maker. $\mathbf{P}_{\pi_{0}}^{\mu}$ and $\mathbf{E}_{\pi_{0}}^{\mu}$ are the probability measure and expectation of the evolution of the observations and Markov state which are strategy dependent. Finally, $\pi_{0}$ denotes the initial distribution of the Markov chain $x$.

In classical quickest detection, the decision policy $\mu$ is a function of the twodimensional belief state (posterior probability mass function) $\pi_{k}(i)=\mathbb{P}\left(x_{k}=\right.$ $\left.i \mid y_{1}, \ldots, y_{k}, u_{1}, \ldots, u_{k-1}\right), i=1,2$, with $\pi_{k}(1)+\pi_{k}(2)=1$. It thus suffices to consider one element, say $\pi_{k}(2)$, of this probability mass function. Classical quickest change detection (see for example [214]) says that the policy $\mu^{*}(\pi)$, which optimizes (2.28), has the following threshold structure: There exists a threshold point $\pi^{*} \in[0,1]$ such that

$$
\mu^{*}\left(\pi_{k}\right)= \begin{cases}2, \text { (continue) } & \text { if } \pi_{k}(2) \geq \pi^{*}, \\ 1, \text { (announce change) } & \text { if } \pi_{k}(2)<\pi^{*} .\end{cases}
$$




\subsubsection{Multi-agent Quickest Detection Problem}

With the above classical formulation in mind, consider now the following multiagent quickest change detection problem: Suppose that a multi-agent system performs social learning to estimate an underlying state according to the social learning protocol of $\$ 2.2 .1$. That is, each agent acts once in a predetermined sequential order indexed by $k=1,2, \ldots$ (Equivalently, as pointed out in the discussion in $\$ 2.2 .1$ a finite number of agents act repeatedly in some pre-defined order and each agent chooses its local decision using the current public belief.) Given these local decisions (or equivalently the public belief), the goal of the global decision maker is to minimize the quickest detection objective (2.28). The problem now is a non-trivial generalization of classical quickest detection. The posterior $\pi$ is now the public belief given by the social learning filter (2.3) instead of a standard Bayesian filter. There is now interaction between the local and global decision makers. The local decision $a_{k}$ from the social learning protocol determines the public belief state $\pi_{k}$ via the social learning filter (2.3), which determines the global decision (stop or continue), which determines the local decision at the next time instant, and so on.

The global decision maker's policy $\mu^{*}: \pi \rightarrow\{1,2\}$ that optimizes the quickest detection objective (2.28) and the cost $J_{\mu^{*}}\left(\pi_{0}\right)$ of this optimal policy are the solution of "Bellman's dynamic programming equation":

$$
\begin{aligned}
\mu^{*}(\pi) & =\operatorname{argmin}\left\{f \pi(2), d(1-\pi(2))+\sum_{a \in \mathcal{A}} V(T(\pi, a)) \sigma(\pi, a)\right\}, \\
V(\pi) & =\min \left\{f \pi(2), d(1-\pi(2))+\sum_{a \in \mathcal{A}} V(T(\pi, a)) \sigma(\pi, a)\right\}, \\
J_{\mu^{*}}\left(\pi_{0}\right) & =V\left(\pi_{0}\right) .
\end{aligned}
$$

Here, $T(\pi, a)$ and $\sigma(\pi, a)$ are given by the social learning filter (2.3) — recall that $a$ denotes the local decision. $V(\pi)$ is called the "value function"-it is the cost incurred by the optimal policy when the initial belief state (prior) is $\pi$. The above problem is more complex than a standard partially observed Markov decision process (POMDP) since the belief state update $T(\pi, a)$ now involves the social learning filter instead of the standard hidden Markov model filter. In particular, as we will illustrate in the numerical example below, unlike in POMDPs, $V(\pi)$ is no longer concave. Also the optimal policy $\mu^{*}(\pi)$ has a very different structure compared to the classical quickest detection. 
Example 2.3. We now illustrate the unusual multi-threshold property of the global decision maker's optimal policy $\mu^{*}(\pi)$ in multi-agent quickest detection with social learning.

Consider the social learning model of $\$ 2.2 .1$ with the following parameters: The underlying state is a 2-state Markov chain $x$ with state space $\mathbb{X}=\{1,2\}$ and transition probability matrix

$$
P=\left[\begin{array}{cc}
1 & 0 \\
0.05 & 0.95
\end{array}\right]
$$

Therefore, the change time $\tau^{0}$ (i.e., the time the Markov chain jumps from state 2 into absorbing state 1) is geometrically distributed with $E\left\{\tau^{0}\right\}=1 / 0.05=20$.

Social Learning Parameters: Individual agents observe the Markov chain $x$ in noise with the observation symbol set $\mathbb{Y}=\{1,2\}$. Suppose the observation likelihood matrix with elements $B_{i y}=\mathbb{P}\left(y_{k}=y \mid x_{k}=i\right)$ is

$$
B=\left[\begin{array}{ll}
0.9 & 0.1 \\
0.1 & 0.9
\end{array}\right]
$$

Agents can choose their local actions a from the action set $\mathcal{A}=\{1,2\}$. The state-dependent cost matrix of these actions is

$$
c=(c(i, a), i \in X, a \in \mathcal{A})=\left[\begin{array}{cc}
4.57 & 5.57 \\
2.57 & 0
\end{array}\right] \text {. }
$$

Agents perform social learning with the above parameters. The intervals $\left[0, \pi_{1}^{*}\right]$ and $\left[\pi_{2}^{*}, 1\right]$ in Figure 2.3 a are regions where the optimal local actions taken by agents are independent of their observations. For $\pi(2) \in\left[\pi_{2}^{*}, 1\right]$, the optimal local action is 2 and, for $\pi(2) \in\left[0, \pi_{1}^{*}\right]$, the optimal local action is 1 . Therefore, individual agents herd for belief states in these intervals (see the definition in $\$ 2.2 .3)$ and the local actions do not yield any information about the underlying state. Moreover, the interval $\left[0, \pi_{1}^{*}\right]$ depicts a region where all agents herd ${ }^{12}$ (again see the definition in $\$ 2.2 .3$ ), meaning that once the belief state is in this region, it remains so indefinitely and all agents choose the same local action 1.

Global Decision Making: Based on the local actions of the agents performing social learning, the global decision maker needs to perform quickest change

\footnotetext{
${ }^{12}$ Note that even if the agent $k$ herds so that its action $a_{k}$ provides no information about its private observation $y_{k}$, the public belief still evolves according to the predictor $\pi_{k+1}=P^{\prime} \pi_{k}$. Therefore, an information cascade does not occur in this example.
} 


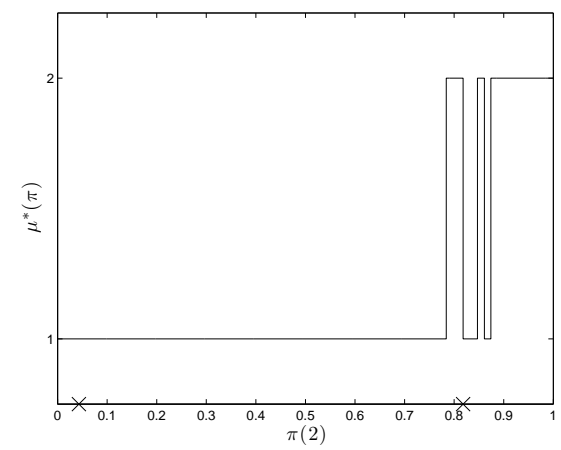

(a) Optimal global decision policy $\mu^{*}(\pi)$

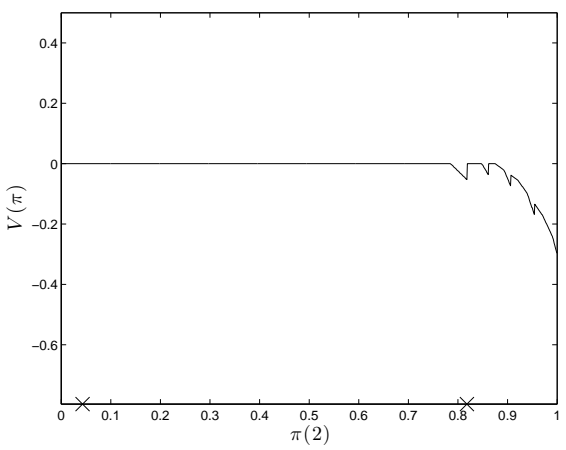

(b) Value function for global decision policy

Figure 2.3: Optimal global decision policy for social learning based quickest time change detection for geometric distributed change time. The parameters are specified in $\$ 2.3$. The optimal policy $\mu^{*}(\pi) \in\{1$ (announce change), 2 (continue) $\}$ is characterized by a triple threshold - that is, it switches from 1 to 2 three times as the posterior $\pi(2)$ increases. As explained in the text, for $\pi(2) \in\left[0, \pi_{1}^{*}\right]$, all agents herd, while for $\pi(2) \in\left[\pi_{2}^{*}, 1\right]$ individual agents herd.

detection. The global decision maker uses the delay penalty $d=1.05$ and false alarm penalty $f=3$ in the objective function (2.28). The optimal policy $\mu^{*}(\pi)$ of the global decision maker where $\pi=[1-\pi(2), \pi(2)]^{\prime}$ is plotted versus $\pi(2)$ in Figure 2.3a. Note $\pi(2)=1$ means that no change has occurred with certainty, while $\pi(2)=0$ means a change has occurred with certainty. The policy $\mu^{*}(\pi)$ was computed by constructing a uniform grid of 1000 points for $\pi(2) \in[0,1]$ and then implementing the dynamic programming equation (2.30) via a fixed point value iteration algorithm for 200 iterations. The horizontal axis $\pi(2)$ is the posterior probability of no change. The vertical axis denotes the optimal decision: $u=1$ denotes stop and declare change, while $u=2$ denotes continue.

The most remarkable feature of Figure 2.3a is the multi-threshold behavior of the global decision maker's optimal policy $\mu^{*}(\pi)$. Recall $\pi(2)$ depicts the posterior probability of no change. Consider the region where $\mu^{*}(\pi)=2$ and sandwiched between two regions where $\mu^{*}(\pi)=1$. Then, as $\pi(2)$ (posterior probability of no change) increases, the optimal policy switches from $\mu^{*}(\pi)=2$ to $\mu^{*}(\pi)=1$. In other words, the optimal global decision policy "changes its mind"-it switches from no change to change as the posterior probability of a 
change decreases! Thus, the global decision (stop or continue) is a non-monotone function of the posterior probability obtained from local decisions.

Figure $2.3 b$ shows the associated value function obtained via stochastic $d y$ namic programming (2.30). Recall that the value function $V(\pi)$ is the cost incurred by the optimal policy with initial belief state $\pi$. Unlike standard sequential detection problems, in which the value function is concave, the figure shows that the value function is non-concave and discontinuous. To summarize, Figure 2.3 shows that social learning based quickest detection results in fundamentally different decision policies compared to classical quickest time detection (which has a single threshold). Thus, making global decisions (stop or continue) based on local decisions (from social learning) is non-trivial. In [154], a detailed analysis of the problem is given together with the characterization of this multi-threshold behavior. More general phase-distributed change times are further considered in [154].

\subsection{Closing Remarks}

In this chapter, we used social learning as a model for interactive sensing with social sensors. We summarize here some extensions of the social learning framework that are relevant to interactive sensing.

Wisdom of Crowds Surowiecki's book [238] is an excellent popular piece that explains the wisdom-of-crowds hypothesis. The wisdom-of-crowds hypothesis predicts that the independent judgments of a crowd of individuals (as measured by any form of central tendency) will be relatively accurate, even when most of the individuals in the crowd are ignorant and error prone. The book also studies situations (such as rational bubbles) in which crowds are not wiser than individuals.

Collect enough people on a street corner staring at the sky, and everyone who walks past will look up.

Such herding behavior is typical in social learning.

In which order should agents act? In the social learning protocol, we assumed that the agents act sequentially in a pre-defined order. However, in many social networking applications, it is important to optimize the order in which agents 
act. For example, consider an online review site where individual reviewers with different reputations make their reviews publicly available. If a reviewer with high reputation publishes its review first, this review will unduly affect the decision of a reviewer with lower reputation. In other words, if the most senior agent "speaks" first, it will unduly affect the decisions of more junior agents. This could lead to an increase in bias of the underlying state estimate 13 . On the other hand, if the most junior agent is polled first, since its variance is large, several agents will need to be polled in order to reduce the variance. We refer the reader to [207] for an interesting description of who should speak first in a public debate 14 . It turns out that, for two agents, the seniority rule is always optimal for any prior-that is, the senior agent speaks first followed by the junior agent; see [207] for the proof. However, for more than two agents, the optimal order depends on the prior and the observations in general.

Global Games for Coordinating Sensing In the classical Bayesian social learning model of $\$ 2.2$, agents act sequentially in time. The global games model that has been studied in economics during the last two decades, considers multiple agents that act simultaneously by predicting the behavior of other agents. The theory of global games was first introduced in [45] as a tool for refining equilibria in economic game theory; see [192] for an excellent exposition. Global games are an ideal method for decentralized coordination amongst agents. They have been used to model speculative currency attacks and regime change in social systems; see [12, 138, 192].

The most widely studied form of a global game is a one-shot Bayesian game which proceeds as follows: Consider a continuum of agents in which each agent $i$ obtains noisy measurements $y^{i}$ of an underlying state of nature $x$. Assume all agents have the same observation likelihood density $p(y \mid x)$; however, the individual measurements obtained by agents are statistically independent of those obtained by other agents. Based on its observation $y^{i}$, each agent takes an action $a^{i} \in\{1,2\}$ to optimize its expected utility $\mathbf{E}\left\{U\left(a^{i}, \alpha\right) \mid y^{i}\right\}$, where $\alpha \in[0,1]$ de-

\footnotetext{
${ }^{13}$ To quote a recent paper from Haas School of Business, U.C. Berkeley [11]: "In 94\% of cases, groups (of people) used the first answer provided as their final answer... Groups tended to commit to the first answer provided by any group member." People with dominant personalities tend to speak first and most forcefully "even when they actually lack competence".

${ }^{14}$ As described in [207], seniority is considered in the rules of debate and voting in the U.S. Supreme Court. "In the past, a vote was taken after the newest justice to the Court spoke. With the justices voting in order of ascending seniority largely, it was said, to avoid the pressure from long-term members of the Court on their junior colleagues."
} 
notes the fraction of all agents that take action 2. Typically, the utility $U(1, \alpha)$ is set to zero.

For example, suppose $x$ (state of nature) denotes the quality of a social group and $y^{i}$ denotes the measurement of this quality by agent $i$. The action $a^{i}=1$ means that agent $i$ decides not to join the social group, while $a^{i}=2$ means that agent $i$ joins the group. The utility function $U\left(a^{i}=2, \alpha\right)$ for joining the social group depends on $\alpha$, where $\alpha$ is the fraction of people that decide to join the group. In [138], the utility function is chosen as follows: If $\alpha \approx 1$, i.e., too many people join the group, then the utility to each agent is small since the group is too congested and agents do not receive sufficient individual service. On the other hand, if $\alpha \approx 0$, i.e., too few people join the group, then the utility is also small since there is not enough social interaction.

Since each agent is rational, it uses its observation $y^{i}$ to predict $\alpha$, i.e., the fraction of other agents that choose action 2. The main question is then: What is the optimal strategy for each agent $i$ to maximize its expected utility?

It has been shown that, for a variety of measurement noise models (observation likelihoods $p(y \mid x)$ ) and utility functions $U$, the symmetric Bayesian Nash equilibrium of the global game is unique and has a threshold structure in the observation. This means that, given its observation $y^{i}$, it is optimal for each agent $i$ to choose its actions as follows:

$$
a^{i}= \begin{cases}1, & \text { if } y^{i}<y^{*} \\ 2, & \text { if } y^{i} \geq y^{*}\end{cases}
$$

where the threshold $y^{*}$ depends on the prior, noise distribution, and utility function.

In the above example of joining a social group, this result means that, if agent $i$ receives a measurement $y^{i}$ of the quality of the group and $y^{i}$ exceeds a threshold $y^{*}$, then it should join. This is yet another example of simple local behavior (act according to a threshold strategy) resulting in global sophisticated behavior (Bayesian Nash equilibrium). As can be seen, global games provide a decentralized way of achieving coordination amongst social sensors. In [12], the above one-shot Bayesian game is generalized to a dynamic (multi-stage) game operating over a possibly infinite horizon. Such games facilitate modelling the dynamics of how people join, interact, and leave social groups.

The papers [151,152] use global games to model networks of sensors and cognitive radios. In [138], it has been shown that the above threshold structure (2.31) for the Bayesian Nash equilibrium breaks down if the utility function $U(2, \alpha)$ de- 
creases too rapidly due to congestion. The equilibrium structure becomes much more complex and can be described by the following quotation [138]:

"Nobody goes there anymore. It's too crowded" - Yogi Berra 


\section{Chapter 3}

\section{Tracking Degree Distribution in Dynamic Social Networks}

\subsection{Introduction}

Social networks can be viewed as complex sensors that provide information about interacting individuals and an underlying state of nature 1 . In this chapter, we consider a dynamic social network where at each time instant one node can join or leave the network. The probabilities of joining or leaving evolve according to the realization of a finite state Markov chain that represents the state of nature. This chapter presents two results. First, motivated by social network applications, the asymptotic behavior of the degree distribution of the Markov-modulated random graph is analyzed. Second, using noisy observations of nodes' connectivity, a "social sensor" is designed for tracking the underlying state of nature as it evolves over time.

\subsubsection{Motivation}

Why analyze the degree distribution? The degree distribution yields useful information about the connectivity of the random graph [8, 150, 203]. The degree distribution can further be used to investigate the diffusion of information or disease through social networks [184, 249]. The existence of a "giant com-

\footnotetext{
${ }^{1}$ For example, real-time event detection from Twitter posts is investigated in [222] or the early detection of contagious outbreaks via social networks is studied in [60].
} 
ponent' 2 in complex networks can be studied using the degree distribution. The size and existence of a giant component has important implications in social networks in terms of modeling information propagation and spread of human disease [86, 202, 204]. The degree distribution is also used to analyze the "searchability" of a network. The "search" problem arises when a specific node in a network faces a problem (request) whose solution is at other node, namely, destination (e.g., delivering a letter to a specific person, or finding a web page with specific information) [3, 249]. The searchability of a social network [249] is the average number of nodes that need to be accessed to reach the destination. Degree distribution is also used to investigate the robustness and vulnerability of a network in terms of the network response to attacks on its nodes or links [43, 128]. The papers [252, 253] further use degree-dependent tools for classification of social networks.

Social sensors for tracking a Markovian target: Tracking a time-varying parameter that evolves according to a finite-state Markov chain has several applications in target tracking [87], change detection [30], multi-user detection in wireless systems [259], and economics [164]. In this chapter, we consider a dynamic social network where the interactions between nodes evolve over time according to a Markov process that undergoes infrequent jumps (the state of nature). An example of such social networks is the friendship network among residents of a city, where the dynamics of the network change in the event of a large festival. (Further examples are provided in Chapter 4) In this chapter, we introduce Markovmodulated random graphs to mimic social networks where the interactions among nodes evolve over time due to the underlying dynamics (state of nature). These social networks can be used as a social sensor for tracking the underlying state of nature. That is, using noisy measurements of the degree distribution of the network, the jumps in the underlying state of nature can be tracked.

\subsubsection{Main Results}

Markov-modulated random graphs are introduced in $\$ 3.2$. We then provide a degree distribution analysis for such graph in $\$ 3.3$ that allows us to determine the relation between the structure of the network (in terms of connectivity) and the underlying state of nature. Indeed, it will be shown in $\$ 3.3$ that there exists a unique

\footnotetext{
${ }^{2} \mathrm{~A}$ giant component is a connected component with size $O(n)$, where $n$ is the total number of vertices in the graph. If the average degree of a random graph is strictly greater than one, then there exists a unique giant component with probability one [61], and the size of this component can be computed from the expected degree sequence.
} 


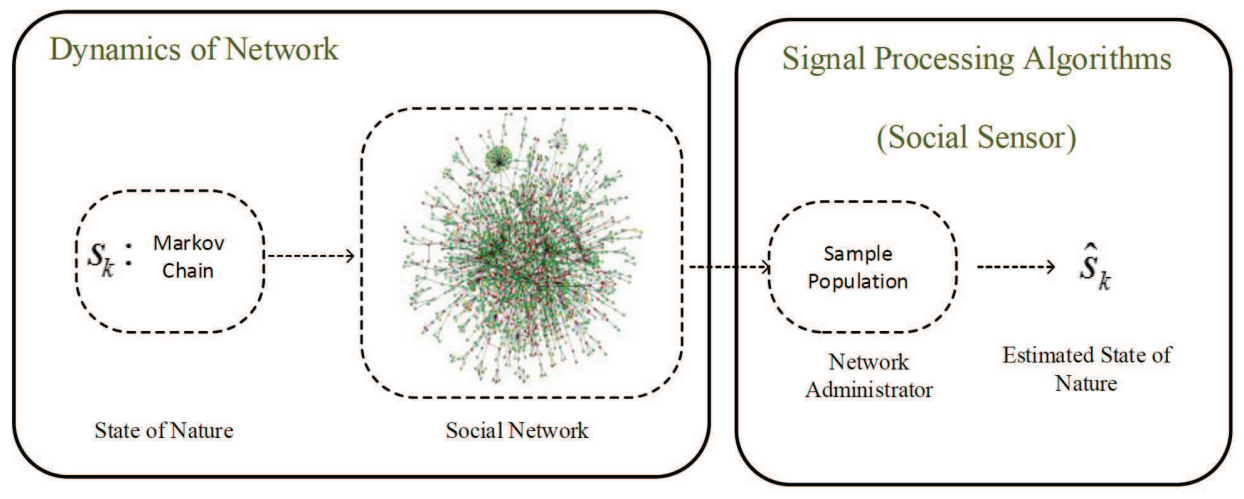

Figure 3.1: Tracking the underlying state of nature using a Markov-modulated random graph as a social sensor.

stationary degree distribution for the Markov-modulated graph for each state of the underlying Markov chain. It thus suffices to estimate the degree distribution in order to track the underlying state of nature. Next, a stochastic approximation algorithm is proposed to track the empirical degree distribution of the Markovmodulated random graph. In particular, we address the following two questions in $\$ 3.5$.

- How can a social sensor estimate (track) the empirical degree distribution using a stochastic approximation algorithm with no knowledge of the Markovian dynamics?

- How accurate are the estimates generated by the stochastic approximation algorithm when the random graph evolves according to the duplicationdeletion model with Markovian switching?

By tracking the degree distribution of a Markov-modulated random graph, we can design a social sensor to track the underlying state of nature using the noisy measurements of nodes' connectivity; see Figure 3.1 .

\subsubsection{Related Works}

For the background and fundamentals on social and economic networks, we refer to [132]. The reader is also referred to [167] for a comprehensive development 
of stochastic approximation algorithms. Adaptive algorithms and stochastic approximations are used in a wide range of applications such as system identification, control theory, adaptive filtering, state estimation, and wireless communications [188, 196, 69]. Tracking capability of regime switching stochastic approximation algorithms is further investigated in [260] in terms of the mean square error. Different applications of social sensors in detection and estimation are investigated in [222, 60, 7]. The differences between social sensors, social sensing, and pervasive sensors along with challenges and open areas in social sensors are further presented in [221].

Here, the related literature on dynamic social networks is reviewed briefly. The book [74] provides a detailed exposition of random graphs. The dynamics of random graphs are investigated in the mathematics literature, for example, see [61, 84, 178] and the reference therein. In [211], a duplication model is proposed where at each time step a new node joins the network. However, the dynamics of this model do not evolve over time. In [61], it is shown that the degree distribution of such networks satisfies a power law. In random graphs which satisfy the power law, the number of nodes with an specific degree depends on a parameter called "power law exponent" [133, 250]. A generalized Markov graph model for dynamic social networks along with its application in social network synthesis and classification is also presented in [253].

\section{Experimental Studies:}

1) Degree distribution analysis: The degree distribution analysis of real-world networks has attracted much attentions recently, [6, 64, 77, 105, 204, 201, 148]. A large network dataset collection can be found in [174], which includes datasets from social networks, web graphs, road networks, internet networks, citation networks, collaboration networks, and communication networks. The paper [201] investigates the structure of scientific collaboration networks in terms of degree distribution, existence of giant component, and the average degree of separation. In the scientific collaboration networks, two scientists are connected if they have co-authored a paper. Another example is the degree distribution of the affiliation network of actors.

2) Social Networks as Social Sensors: With a large number of rational agents, social networks can be viewed as social sensors for extracting information about the world or people. For example, the paper [222] presents a social sensor (based on Twitter) for real-time event detection of earthquakes in Japan, namely, the target event. They perform semantic analysis on tweets (which are related to the 
target event) in order to detect the occurrence and the location of the target event (earthquake). Another example is the use of the social network as a sensor for early detection of contagious outbreaks [60]. Using the fact that central individuals in a social network are likely to be infected sooner than others, a social sensor is designed for the early detection of contagious outbreaks in [60]. The performance of this social sensor was verified during a flu outbreak at Harvard College in 2009-the experimental studies showed that this social sensor provides a significant additional time to react to epidemics in the society.

\subsection{Markov-Modulated Random Graphs}

To mimic dynamic social networks, we consider Markov-modulated random graphs of the duplication-deletion type. Let $n=0,1,2, \ldots$ denote discrete time. Denote by $\theta_{n}$ a discrete-time Markov chain with state space

$$
\mathcal{M}=\{1,2, \ldots, M\},
$$

evolving according to the $M \times M$ transition probability matrix

$$
A^{\rho}=I+\rho Q
$$

and initial probability distribution $\pi_{0}$. Here, $I$ is an $M \times M$ identity matrix, $\rho$ is a small positive real number, and $Q$ is an irreducible generator of a continues-time Markov chain satisfying

$$
q_{i j}>0, \text { for } i \neq j, \text { and } Q \mathbf{1}=\mathbf{0}
$$

where 1 and 0 represent column vectors of ones and zeros, respectively. The transition probability matrix $A^{\rho}$ is therefore close to identity matrix. Here and henceforth, we refer to such a Markov chain $\theta_{n}$ as a "slow" Markov chain.

A Markov-modulated duplication-deletion random graph is parameterized by the 7-tuple $\left(M, A^{\rho}, \pi_{0}, r, p, q, G_{0}\right)$. Here, $p$ and $q$ are $M$-dimensional vectors with elements $p(i)$ and $q(i) \in[0,1], i=1, \ldots, M$, where $p(i)$ denotes the connection probability, and $q(i)$ denotes the deletion probability. Also, $r \in[0,1]$ denotes the probability of duplication step, and $G_{0}$ denotes the initial graph at time 0 . In general, $G_{0}$ can be any finite simple connected graph. For simplicity, we assume that $G_{0}$ is a simple connected graph with size $N_{0}$.

The duplication-deletion random graph ${ }^{3}$ is constructed via Algorithm 2 .

\footnotetext{
${ }^{3}$ For convenience in the analysis, assume that a node generated in the duplication step cannot
} 
$\overline{\text { Algorithm } 2 \text { Markov-modulated Duplication-deletion Graph parameterized by }}$ $\left(M, A^{\rho}, \pi_{0}, r, p, q, G_{0}\right)$

At time $n$, given the graph $G_{n}$ and Markov chain state $\theta_{n}$, simulate the following events:

Step 1: Duplication step: With probability $r$ implement the following steps:

- Choose node $u$ from graph $G_{n}$ randomly with uniform distribution.

- Vertex-duplication: Generate a new node $v$.

- Edge-duplication:

- Connect node $u$ to node $v$. (A new edge between $u$ and $v$ is added to the graph.)

- Connect each neighbor of node $u$ with probability $p\left(\theta_{n}\right)$ to node $v$. These connection events are statistically independent.

Step 2: Deletion Step: With probability $q\left(\theta_{n}\right)$ implement the following steps:

- Edge-deletion: Choose node $w$ randomly from $G_{n}$ with uniform distribution. Delete node $w$ along with the connected edges in graph $G_{n}$.

- Duplication Step: Implement Step 1.

Step 3: Denote the resulting graph by $G_{n+1}$.

Generate $\theta_{n+1}$ (Markov chain) using transition matrix $A^{\rho}$.

Step 4: Network Manager's Diagnostics: The network manager computes the estimates of the expected degree distribution.

Set $n \rightarrow n+1$ and go to Step 1 . 
The Markov-modulated random graph generated by Algorithm 2 mimics social networks where the interactions between nodes evolve over time due to the underlying dynamics (state of nature) such as seasonal variations (e.g., the high school friendship social network evolving over time with different winter/summer dynamics). In such cases, the connection/deletion probabilities $p, q$ depend on the state of nature and evolve with time. Algorithm 2 models these time variations as a finite state Markov chain $\theta_{n}$ with transition matrix $A^{\rho}$.

\section{Discussion:}

The connection/deletion probabilities $p, q$ can be determined by the solution of a utility maximization problem. Let $U^{\text {join }}:[0,1] \times \mathcal{M} \rightarrow \mathbb{R}$ denote a utility function that gives payoff to an individual who considers to expand his neighbors in "Edge-duplication step" of Algorithm 2 as a function of $(p, \theta)$. Similarly, let $U^{\text {leave }}:[0,1] \times \mathcal{M} \rightarrow \mathbb{R}$ denote a utility function that pays off to an individual who considers to leave the network in "Deletion step" of Algorithm 2 as a function of $(q, \theta)$. With the above utility functions, the probabilities of connection/deletion when the state of nature is $\theta$ can be viewed as the solutions of the following maximization problems:

$$
\begin{aligned}
& p(\theta)=\underset{p}{\operatorname{argmax}}\left\{U^{\text {join }}(p, \theta)\right\}, \\
& q(\theta)=\underset{q}{\operatorname{argmax}}\left\{U^{\text {leave }}(q, \theta)\right\} .
\end{aligned}
$$

These utility functions can be interpreted in terms of mutual benefits and privacy concerns. One example could be $U^{\text {join }}(p, \theta)=b^{\text {join }}(p, \theta)-v$, where $b^{\text {join }}(p, \theta)$ is the benefit one obtains by expanding his network with probability $p$ when the underlying state of nature is $\theta$, and $v$ is the cost incurred by sacrificing his "privacy". In this example, when an individual decides to leave the network, the utility he obtains will be $U^{\text {leave }}(q, \theta)=b^{\text {leave }}(q, v)-c(\theta)$, where $b^{\text {leave }}(q, v)$ is the benefit he earns by preserving privacy and $c(\theta)$ is the benefit he loses by leaving the network when the underlying state of nature is $\theta$.

be eliminated in the deletion step immediately after its generation - that is, the new node, which is generated in the vertex-duplication step of Step 2, remains unchanged in Step 3. Also, the nodes whose degrees are changed in the edge-deletion part of Step 3, remain unchanged in the duplication part of Step 3 at that time instant. To prevent the isolated nodes, assume that the neighbor of a node with degree one cannot be eliminated in the deletion step. The duplication step in Step 2 ensures that the graph size does not decrease. 


\subsection{Degree Distribution Analysis of Markov-modulated Random Graphs}

This section presents degree distribution analysis of the fixed size Markov-modulated duplication-deletion random graph generated according to Algorithm 2. As we will show shortly, the expected degree distribution of such a graph depends on the underlying dynamics of the model that follow the state of nature. Therefore, the expected degree distribution of the graph generated can be used to track the state of nature. Therefore, the entire social network forms a social sensor. Before proceeding, let us introduce some notation.

Notation. At each time $n$, let $N_{n}$ denote the number of nodes of graph $G_{n}$. Also, let $f_{n}(i)$ denote the number of vertices of graph $G_{n}$ with degree $i$. Clearly, $\sum_{i \geq 1} f_{n}(i)=N_{n}$. Define the "empirical vertex degree distribution" as

$$
g_{n}(i)=\frac{f_{n}(i)}{N_{n}}, \quad \text { for } 1 \leq i \leq N_{n}
$$

Note that $g_{n}(i)$ can be viewed as a probability mass function since $g_{n}(i) \geq 0$ and $\sum_{i} g_{n}(i)=1$. Let $\bar{g}_{n}=\mathbf{E}\left\{g_{n}\right\}$ denote the "expected vertex degree distribution," where $g_{n}$ is the empirical degree distribution defined in (3.5).

Consider the sequence of finite duplication-deletion random graphs $\left\{G_{n}\right\}$, generated by Algorithm 2 with $r=0$. Clearly, the number of vertices in the graph satisfies $N_{n}=N_{0}$ for $n=1,2, \ldots$, i.e., size of the graph is fixed. The following assumption characterizes the Markovian dynamics of the state of the nature.

Assumption 3.1. The slow Markov chain $\theta_{n}$ evolves according to the transition matrix $A^{\rho}=I+\rho Q$, where $\rho$ is a small positive real number and $Q=\left[q_{i j}\right]$ is an irreducible 4 generator matrix satisfying

$$
q_{i j} \geq 0, \text { if } i \neq j, \text { and } \sum_{j=1}^{M} q_{i j}=0, \forall i
$$

The initial distribution $\pi_{0}$ is further independent of $\rho$.

\footnotetext{
${ }^{4}$ The irreducibility assumption implies that there exists a unique stationary distribution $\pi \in$ $\mathbb{R}^{M \times 1}$ for this Markov chain such that $\pi^{\prime}=\pi^{\prime} A^{\rho}$.
} 
Theorem 3.3.1 below asserts that the expected degree distribution of the fixed size Markov-modulated duplication-deletion random graph satisfies a recursive equation. Using this recursive equation, one can solve for the expected degree distribution.

Theorem 3.3.1. Consider the fixed size Markov-modulated duplication-deletion random graph generated according to Algorithm 2 where $A^{\rho}=I+\rho Q$ and $r=0$. Let $\bar{g}_{n}^{\theta}=\mathbf{E}\left\{g_{n} \mid \theta_{n}=\theta\right\}$ denote the expected degree distribution of nodes when the state of the underlying Markov chain is $\theta_{n}=\theta$. Then, $\bar{g}_{n}^{\theta}$ satisfies the following recursion

$$
\bar{g}_{n+1}^{\theta}=\left(I+\frac{1}{N_{0}} L^{\prime}(\theta)\right) \bar{g}_{n}^{\theta}
$$

where $C^{\prime}$ denotes transpose of a matrix $C$, and $L(\theta)$ is a generator matrix 5 with elements $\left(\right.$ for $\left.1 \leq i, j \leq N_{0}\right)$ :

$$
l_{j i}(\theta)= \begin{cases}0, & j<i-1 \\
q(\theta) p(\theta)^{i-1}+q(\theta)(1+p(\theta)(i-1)), & j=i-1 \\
i q(\theta) p(\theta)^{i-1}(1-p(\theta))-q(\theta)(i+2+p(\theta) i), & j=i, \\
q(\theta)\left(\begin{array}{l}
i+1 \\
i-1
\end{array}\right) p(\theta)^{i-1}(1-p(\theta))^{2}+q(\theta)(i+1), & j=i+1 \\
q(\theta)\left(\begin{array}{c}
j \\
i-1
\end{array}\right) p(\theta)^{i-1}(1-p(\theta))^{j-i+1}, & j>i+1\end{cases}
$$

Proof. The proof is presented in Appendix A.1.

Theorem 3.3.1 shows that evolution of the expected degree distribution in a fixed size Markov-modulated duplication-deletion random graph satisfies (3.6). One can rewrite (3.6) as

$$
\bar{g}_{n+1}^{\theta}=B_{N_{0}}^{\prime}(\theta) \bar{g}_{n}^{\theta}
$$

where $B_{N_{0}}(\theta)=I+\frac{1}{N_{0}} L(\theta)$. Since $L(\theta)$ is a generator matrix, $B_{N_{0}}(\theta)$ can be considered as the transition matrix of a slow Markov chain. It is also straightforward to show that for each $\theta \in \mathcal{M}, B_{N_{0}}(\theta)$ is irreducible and aperiodic. Hence, for each state of the Markov chain $\theta \in \mathcal{M}$, there exists a unique stationary distribution $\bar{g}(\theta)$ such that

$$
\bar{g}(\theta)=B_{N_{0}}^{\prime}(\theta) \bar{g}(\theta)
$$

Note that the underlying Markov chain $\left\{\theta_{n}\right\}$ depends on the small parameter $\rho$. The main idea is that, although $\theta_{n}$ is time-varying but it is piecewise constant (since $\rho$ is small parameter) — it changes slowly over time. Further, in light

\footnotetext{
${ }^{5}$ That is, each row adds to zero and each non-diagonal element of $L(\theta)$ is positive.
} 
of (3.6), the evolution of $\bar{g}_{n}^{\theta}$ depends on $\frac{1}{N_{0}}$. Our assumption throughout this chapter is that $\rho \ll \frac{1}{N_{0}}$. Therefore, the evolution of $\bar{g}_{n}^{\theta}$ is faster than the evolution of $\theta_{n}$. That is, $\bar{g}_{n}^{\theta}$ reaches its stationary distribution $\bar{g}(\theta)$ before the state of $\theta_{n}$ changes. From (3.9), the expected degree distribution of the fixed size Markov-modulated duplication-deletion random graph can be uniquely computed for each state of the underlying Markov chain $\theta_{n}=\theta$. This allows us to track the state of nature $\theta_{n}$ via estimating the expected degree distribution as will be shown in $\$ 3.5$.

\subsection{Case Study: Power Law Analysis of Infinite Graph}

So far in this chapter, a degree distribution analysis is provided for the fixed size Markov-modulated random graph generated according to Algorithm 2 with $r=0$. Motivated by applications in social networks, this section extends this analysis to the infinite duplication-deletion random graphs without Markovian dynamics. Here, we investigate the random graph generated according to Algorithm 2 with $r=1$, and when there are no Markovian dynamics $(M=1)$. Since $r=1$ for $n \geq 0, G_{n+1}$ has an extra vertex as compared to $G_{n}$. In particular, since $G_{0}$ is an empty set, $G_{n}$ has $n$ nodes, i.e., $N_{n}=n$. In the rest of this section, employing the same approach as in the proof of Theorem 3.3.1, it will be shown that the infinite duplication-deletion random graph without Markovian dynamics satisfies a power law. An expression is further derived for the power law exponent.

Let us first define the power law:

Definition 3.1 (Power Law). Consider the infinite duplication-deletion random graph without Markovian dynamics generated according to Algorithm 2 Let $n_{k}$ denote the number of nodes of degree $k$ in a random graph $G_{n}$. Then, $G_{n}$ satisfies a power law distribution if $n_{k}$ is proportional to $k^{-\beta}$ for a fixed $\beta>1$, i.e.,

$$
\log n_{k}=\alpha-\beta \log k
$$

where $\alpha$ is a constant. $\beta$ is called the power law exponent.

The power law distribution is satisfied in many networks such as WWWgraphs, peer-to-peer networks, phone call graphs, co-authorship graph and various massive online social networks (e.g. Yahoo, MSN, Facebook) [24, 34, 63, 88, 145, 232, 236]. The power law exponent describes asymptotic degree distribution of networks from which characteristics of networks such as maximum degree, searchability, existence of giant component, and diameter of the graph can 
be investigated [61, 249]. The following theorem states that the graph generated according to Algorithm 2 with $r=1$ and $M=1$ satisfies a power law.

Theorem 3.4.1. Consider the infinite random graph with Markovian dynamics $G_{n}$ obtained by Algorithm 2 with 7-tuple $\left(1,1,1,1, p, q, G_{0}\right)$ with the expected degree distribution $\bar{g}_{n}$. As $n \rightarrow \infty, G_{n}$ satisfies a power law. That is,

$$
\lim _{n \rightarrow \infty} \log \bar{g}_{n}(i)=\alpha-\beta \log i
$$

where the power law exponent, $\beta$, can be computed from

$$
(1+q)\left(p^{\beta-1}+p \beta-p\right)=1+\beta q .
$$

Here, $p$ and $q$ are the probabilities defined in duplication and deletion steps, respectively.

Proof. The detailed proof is provided in [114]. Here, we only present an outline of the proof that comprises of two steps: (i) finding the power law exponent, and (ii) showing that the degree distribution converges to a power law with the computed compnent as $n \rightarrow \infty$. To find the power law exponent, we derive a recursive equation for the number of nodes with degree $i+1$ at time $n+1$, denoted by $f_{n+1}(i+1)$, in terms of the degrees of nodes in graph $G_{n}$. Then, rearranging this recursive equation yields an equation for the power law exponent. To prove that the degree distribution satisfies a power law, we define a new parameter $h_{n}(i)=$ $\frac{1}{n} \sum_{k=1}^{i} \mathbf{E}\left\{f_{n}(k)\right\}$ and show that $\lim _{n \rightarrow \infty} h_{n}(i)=\sum_{k=1}^{i} C k^{-\beta}$, where $\beta$ is the power law exponent computed in the first step.

Theorem 3.4.1 asserts that the infinite duplication-deletion random graph without Markovian dynamics generated by Algorithm 2 satisfies a power law and provides an expression for the power law exponent. The significance of this theorem is that it ensures, with use of one single parameter (the power law exponent), we can describe the degree distribution of large numbers of nodes in graphs that model social networks. The above result slightly extends [62, 211], where only a duplication model is considered. However, a graph generated by any arbitrary pure duplication step may not satisfy the power law6. Theorem 3.4.1 allows us to explore characteristics (such as searchability, diffusion, and existence/size of the giant component) of large networks which can be modeled with the infinite duplication-deletion random graphs.

\footnotetext{
${ }^{6}$ Bebek et al. in [25] provide conditions on the dynamics of the duplication process such that the resulting graph satisfies a power law.
} 


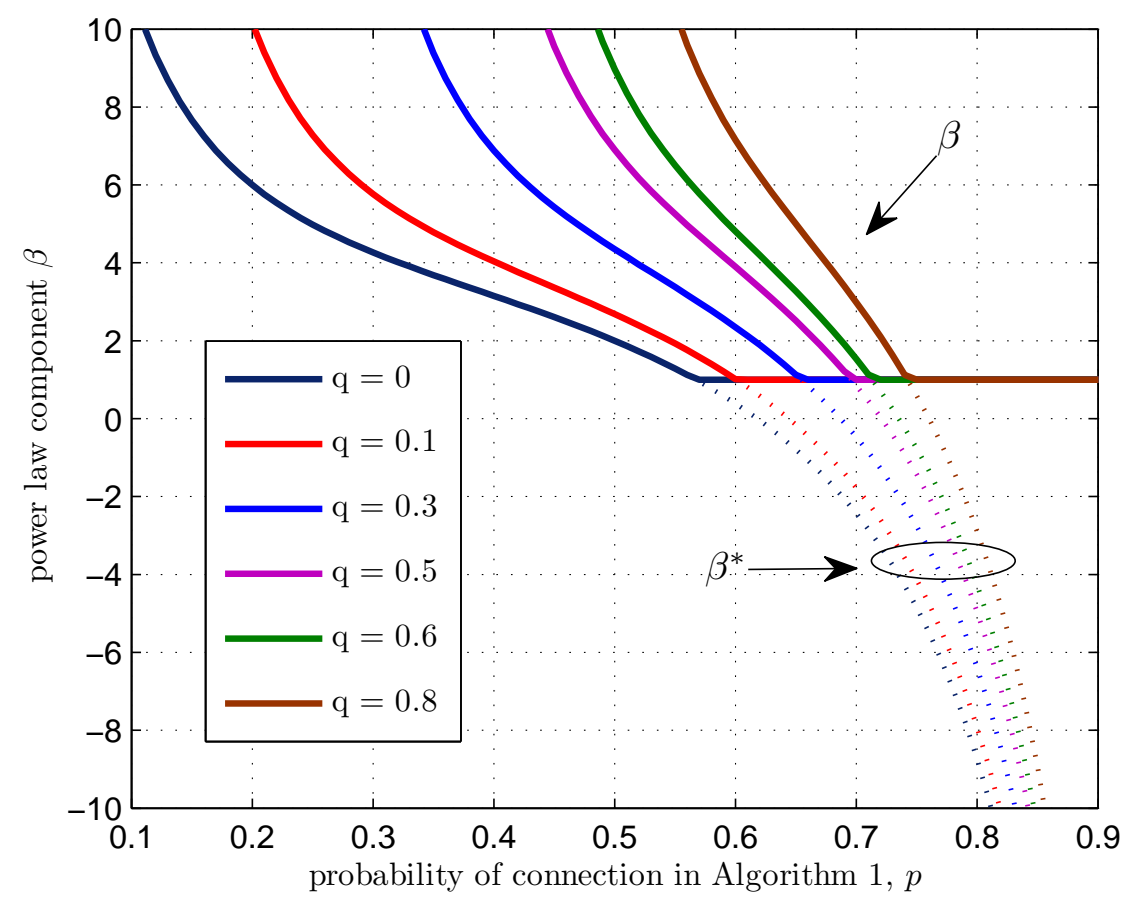

Figure 3.2: The power law exponent for the non-Markovian random graph generated according to Algorithm 2] obtained by (3.11) for different values of $p$ and $q$ in Algorithm 2,

Remark 3.1 (Power Law Exponent). Let $\beta^{*}$ denote the solution of (3.11). Then, the power law exponent is defined as $\beta=\max \left\{1, \beta^{*}\right\}$. Figure 3.2 shows the power law exponent and $\beta^{*}$ versus $p$ for different values of probability of deletion q. As can be seen in Figure 3.2 the power law exponent is increasing in $q$ and decreasing in $p$.

\subsection{Social Network as a Social Sensor}

In $\$ 3.3$, a degree distribution analysis has been provided for the fixed size Markovmodulated duplication-deletion random graph generated by Algorithm 2 , It was further shown in $\$ 3.3$ that there exists a unique stationary degree distribution for each state of the underlying Markov chain $\left\{\theta_{n}\right\}$ which represents state of nature. 
In this section, we assume that the empirical degree distribution of the graph, $g_{n}$, is observed in noise. Motivated by social sensor applications, the aim here is to track the unknown state of nature-without knowing the dynamics of the graph-using noisy observations of degree distribution. Since the expected degree distribution of the Markov-modulated duplication-deletion random graph depends on the underlying Markov chain, the state of nature can be estimated via tracking the expected degree distribution; see (3.7). Here, the social network is used to track the underlying state of nature. Therefore, the entire social network forms a social sensor.

The degree distribution of the network generated according to Algorithm 2 is measured in noise as follows

$$
\widehat{f}_{n}=f_{n}+\omega_{n}
$$

Here, at each time $n$, the elements $\omega_{n}(i)$ of the noise vector are integer-valued zero mean random variables, and $\sum_{i \geq 1} \omega_{n}(i)=0$. The zero sum assumption ensures that $\widehat{f}_{n}$ is a valid empirical distribution. The observation process can be viewed as counting the number of nodes with specific degree by the administrator of the network. In terms of the empirical vertex distribution, one can rewrite this measurement process as

$$
y_{n}(i)=\frac{\widehat{f}_{n}(i)}{\sum_{i \geq 0} \widehat{f}_{n}(i)}=\frac{\widehat{f}_{n}(i)}{N_{0}}=g_{n}(i)+\frac{1}{N_{0}} \omega_{n}(i) .
$$

That is, the vertex distribution $g_{n}$ of the graph $G_{n}$ is measured in noise:

$$
y_{n}=g_{n}+e_{n}
$$

where $e_{n}=\frac{\omega_{n}}{N_{0}}$. Recall that $N_{n}=N_{0}$ when $r=0$. The normalized noisy observations $y_{n}$ are used to estimate the empirical probability mass function of the degree of each node. To estimate a time-varying probability mass function (pmf), the following stochastic approximation algorithm with (small positive) constant step-size $\varepsilon$ is used

$$
\widehat{g}_{n+1}=\widehat{g}_{n}+\varepsilon\left(y_{n}-\widehat{g}_{n}\right) .
$$

Note that the stochastic approximation algorithm (3.14) does not assume any knowledge of the Markov-modulated dynamics of the graph (state of nature). The Markov chain assumption for the random graph dynamics is only used in our convergence and tracking analysis. By means of the stochastic approximation (3.14), 
the social sensor can track the expected degree distribution and, consequently, the underlying state of nature.

Example 3.1. Consider the scenario that the underlying Markov chain (state of nature) has $M=2$ states with slow transition matrix $A^{\rho}=I+\rho Q$. Assume that the size of the graph $N_{0}$ is sufficiently large such that the evolution of $\bar{g}_{n}^{\theta}$ is faster than the evolution of $\theta_{n}$. This means that the expected degree distribution $\bar{g}_{n}^{\theta}$ reaches its stationary distribution $(\bar{g}(\theta))$ before the state of $\theta_{n}$ changes. The stationary distribution in this example, which can be computed from (3.6), can be either $\bar{g}(1)$ (if the state of nature is $\theta=1$ ) or $\bar{g}(2)$ (if the state of nature is $\theta=2$ ). Assume that there exist a network administrator who has access to noisy measurements of nodes' degrees $y_{n}$ (e.g., by counting the number of nodes with specific degrees). Then, by use of the stochastic approximation algorithm (3.14), the network administrator is able to precisely track the expected degree distribution. From the estimated degree distribution, the network administrator can estimate the current state of nature. In this example, the social network is used to estimate the unknown state of nature from noisy observations recorded from the network, therefore, can be viewed as a social sensor. In the next subsection, we show that the distance from the expected degree distribution and the estimates obtained by stochastic approximation algorithm (3.14) is bounded.

\subsubsection{Tracking Error of the Stochastic Approximation Algo- rithm}

The goal here is to analyze how well the algorithm tracks the empirical degree distribution of the graph (and consequently the state of nature). To this end, we analyze the asymptotic behavior of the estimated degree distribution. Define the tracking error as $\widetilde{g}_{n}=\widehat{g}_{n}-\bar{g}\left(\theta_{n}\right)$. Theorem 3.5.1 below shows that the difference between the sample path and the expected probability mass function is smallimplying that the stochastic approximation algorithm can successfully track the Markov-modulated node distribution given the noisy measurements. We again emphasize that no knowledge of the Markov chain parameters are required in the algorithm. It also finds the order of this difference in terms of $\varepsilon$ and $\rho$.

Theorem 3.5.1. Consider the random graph $\left(M, A^{\rho}, \pi_{0}, p, q, r, G_{0}\right)$. Suppose 7 that $\rho^{2}=o(\varepsilon)$. Then, for sufficiently large $n$, the tracking error of the stochastic

\footnotetext{
${ }^{7}$ In this chapter, we assume that $\rho=O(\varepsilon)$. Therefore, $\rho^{2}=o(\varepsilon)$.
} 
approximation algorithm (3.14) satisfies

$$
\mathbf{E}\left|\widetilde{g}_{n}\right|^{2}=O\left(\varepsilon+\rho+\frac{\rho^{2}}{\varepsilon}\right) .
$$

Proof. The proof uses the perturbed Lyapunov function method and is provided in Appendix A.2.

As a corollary of Theorem 3.5.1, we obtain the following mean square error convergence result.

Corollary 3.1. Under the conditions of Theorem 3.5.1 if $\rho=O(\varepsilon)$,

$$
\mathbf{E}\left|\widetilde{g}_{n}\right|^{2}=O(\varepsilon)
$$

Therefore,

$$
\limsup _{\varepsilon \rightarrow 0} \mathbf{E}\left|\widetilde{g}_{n}\right|^{2}=0
$$

\subsubsection{Limit System Characterization}

The following theorem asserts that the sequence of estimates generated by the stochastic approximation algorithm (3.14) follows the dynamics of a Markovmodulated ordinary differential equation (ODE).

Before proceeding with the main theorem below, let us recall a definition.

Definition 3.2 (Weak Convergence). Let $Z_{k}$ and $Z$ be $\mathbb{R}^{r}$-valued random vectors. We say $Z_{k}$ converges weakly to $Z\left(Z_{k} \Rightarrow Z\right)$ if for any bounded and continuous function $f(\cdot), E f\left(Z_{k}\right) \rightarrow \operatorname{Ef}(Z)$ as $k \rightarrow \infty$.

Weak convergence is a generalization of convergence in distribution to a function space 8 .

Theorem 3.5.2. Consider the Markov-modulated random graph generated according to Algorithm 2 and the sequence of estimates $\left\{\widehat{g}_{n}\right\}$, generated by the stochastic approximation algorithm (3.14). Suppose Assumption 3.1 holds and $\rho=O(\varepsilon)$. Define the continuous-time interpolated process

$$
\widehat{g}^{\varepsilon}(t)=\widehat{g}_{n}, \theta^{\varepsilon}(t)=\theta_{n} \text { for } t \in[n \varepsilon,(n+1) \varepsilon) .
$$

\footnotetext{
${ }^{8}$ We refer the interested reader to [167, Chapter 7] for further details on weak convergence and related matters. Appendix A.3 contains a brief outline.
} 
Then, as $\varepsilon \rightarrow 0,\left(\widehat{g}^{\varepsilon}(\cdot), \theta^{\varepsilon}(\cdot)\right)$ converges weakly to $(\widehat{g}(\cdot), \theta(\cdot))$, where $\theta(\cdot)$ is a continuous-time Markov chain with generator $Q, \widehat{g}(\cdot)$ satisfies the Markovmodulated $O D E$

$$
\frac{d \widehat{g}(t)}{d t}=-\widehat{g}(t)+\bar{g}(\theta(t)), \quad \widehat{g}(0)=\widehat{g}_{0}
$$

and $\bar{g}(\theta)$ is defined in (3.9).

The above theorem asserts that the limit system associated with the stochastic approximation algorithm (3.14) is a Markovian switched ODE (3.17). As mentioned in $\S 3.3$, this is unusual since typically in the averaging theory analysis of stochastic approximation algorithms, convergence occurs to a deterministic ODE. The intuition behind this somewhat unusual result is that the Markov chain evolves on the same time-scale as the stochastic approximation algorithm. If the Markov chain evolved on a faster time-scale, then the limiting dynamics would indeed be a deterministic ODE weighed by the stationary distribution of the Markov chain. If the Markov chain evolved slower than the dynamics of the stochastic approximation algorithm, then the asymptotic behavior would also be a deterministic ODE with the Markov chain being a constant.

\subsubsection{Scaled Tracking Error}

Next, we study the behavior of the scaled tracking error between the estimates generated by the stochastic approximation algorithm (3.14) and the expected degree distribution. The following theorem states that the tracking error should also satisfy a switching diffusion equation and provides a functional central limit theorem for this scaled tracking error. Let $\nu_{k}=\frac{\widehat{g}_{k}-\mathbf{E}\left\{\bar{g}\left(\theta_{k}\right)\right\}}{\sqrt{\varepsilon}}$ denote the scaled tracking error.

Theorem 3.5.3. Suppose Assumption 3.1 holds. Define $\nu^{\varepsilon}(t)=\nu_{k}$ for $t \in$ $[k \varepsilon,(k+1) \varepsilon)$. Then, $\left(\nu^{\varepsilon}(\cdot), \theta^{\varepsilon}(\cdot)\right)$ converges weakly to $(\nu(\cdot), \theta(\cdot))$ such that $\nu(\cdot)$ is the solution of the following Markovian switched diffusion process

$$
\nu(t)=-\int_{0}^{t} \nu(s) d s+\int_{0}^{t} \Sigma^{\frac{1}{2}}(\theta(\tau)) d \omega(\tau) .
$$

Here, $\omega(\cdot)$ is an $\mathbb{R}^{N_{0}}$-dimensional standard Brownian motion. The covariance matrix $\Sigma(\theta)$ in (3.18) can be explicitly computed as

$$
\Sigma(\theta)=Z(\theta)^{\prime} D(\theta)+D(\theta) Z(\theta)-D(\theta)-\bar{g}(\theta) \bar{g}^{\prime}(\theta) .
$$


Here, $D(\theta)=\operatorname{diag}\left(\bar{g}(\theta, 1), \ldots, \bar{g}\left(\theta, N_{0}\right)\right)$ and $Z(\theta)=\left(I-B_{N_{0}}(\theta)+\mathbf{1} \bar{g}^{\prime}(\theta)\right)^{-1}$, where $B_{N_{0}}\left(\theta_{n}\right)$ and $\bar{g}(\theta)$ are defined in (3.3) and (3.9), respectively.

For general switching processes, we refer to [263]. In fact, more complex continuous-state dependent switching rather than Markovian switching are considered there. Equation (3.19) reveals that the covariance matrix of the tracking error depends on $B_{N_{0}}(\theta)$ and $\bar{g}(\theta)$ and, consequently, on the parameters $p$ and $q$ of the random graph. Recall from $\$ 3.3$ that $B_{N_{0}}(\theta)$ is the transition matrix of the Markov chain which models the evolution of the expected degree distribution in Markov modulated random graphs and can be computed from Theorem 3.3.1. The covariance of the tracking error, which can be explicitly computed from (3.19), is useful for computational purposes.

\subsection{A Note on Degree-based Graph Construction}

The first step in numerical studies of social networks is the graphical modeling of such networks. A graph can be uniquely determined by the adjacency matrix (also known as the connectivity matrix) of the graph. However, in the graphical modeling of social networks (specially when the size of the network is relatively large), the only available information is the degree sequence of nodes, and not the adjacency matrix of the network.

Definition 3.3. The degree sequence, denoted by $\mathrm{d}$, is a non-increasing sequence comprising of the vertex degrees of the graph vertices.

The degree sequence, in general, does not specify the graph uniquely; there can be a large number of graphs that realize a given degree sequence. It is straightforward to show that not all integer sequences represent a true degree sequence of a graph. For example, sequence $\mathbf{d}=\{2,1,1\}$ represents a tree with two edges, but $\mathbf{d}=\{3,2,1\}$ cannot be realized as the degree sequence of a simple graph. Motivated by social network applications, this section addresses the following two questions given a degree sequence $\mathbf{d}$ :

- Existence Problem: Is there any simple graph that realizes $\mathbf{d}$ ?

- Construction Problem: How can we construct all simple graphs that realize a true degree sequence $\mathrm{d}$ ? 
There are two well-known results that address the existence problem: (i) the Erdös-Gallai theorem [82] and the Havel-Hakimi theorem [123, 111]. These theorems provide necessary and sufficient conditions for a sequence of non-negative integers to be a true degree sequence of a simple graph. Here, we recall these results without proofs.

Theorem 3.6.1 (Erdös-Gallai, [82]). Let $d_{1} \geq d_{2} \geq \cdots \geq d_{n}>0$ be integers. Then, the degree sequence $\mathbf{d}=\left\{d_{1}, \cdots, d_{n}\right\}$ is graphical if and only if

1. $\sum_{i=1}^{n} d_{i}$ is even;

2. for all $1 \leq k<n$ :

$$
\sum_{i=1}^{k} d_{i} \leq k(k-1)+\sum_{i=k+1}^{n} \min \left\{k, d_{i}\right\} .
$$

It is shown in [244] that there is no need to check (3.20) for all $1 \leq k \leq n-1$; it suffices to check (3.20) for $1 \leq k \leq s$, where $s$ is chosen such that $d_{s} \geq s$ and $d_{s+1}<s+1$. Note that, in degree-based graph construction, we only care about nodes of degree greater than zero; zero-degree nodes are isolated nodes which can be added to the graph consisting of nodes of degree greater than zero.

The Havel-Hakimi theorem also provides necessary and sufficient conditions for a degree sequence to be graphical. It also gives a greedy algorithm to construct a graph from a given graphical degree sequence.

Theorem 3.6.2 (Havel-Hakimi, [123, 111]). Let $d_{1} \geq d_{2} \geq \cdots \geq d_{n}>0$ be integers. Then, the degree sequence $\mathbf{d}=\left\{d_{1}, \cdots, d_{n}\right\}$ is graphical if and only if the degree sequence $\mathbf{d}^{\prime}=\left\{d_{2}-1, d_{3}-1, \cdots, d_{d_{1}+1}-1, d_{d_{1}+2}, \cdots, d_{n}\right\}$ is graphical.

In the following, we provide algorithms to construct a simple graph from a true degree sequence. In the construction problem, the degree sequence is treated as a collection of half-edges; a node with degree $d_{i}$ has $d_{i}$ half-edges. One end of these half-edges are fixed at node $i$, but the other ends are free. An edge between node $i$ and node $j$ is formed by connecting a half-edge from node $i$ to a halfedge from node $j$. The aim is to connect all these half edges such that no free half-edge is left. The Havel-Hakimi theorem provides a recursive procedure to construct a graph from a graphical degree sequence. This procedure is presented in Algorithm 3

Using Algorithm 3, one can sample from graphical realizations of a given degree sequence. In this algorithm, each vertex is first connected to nodes with 


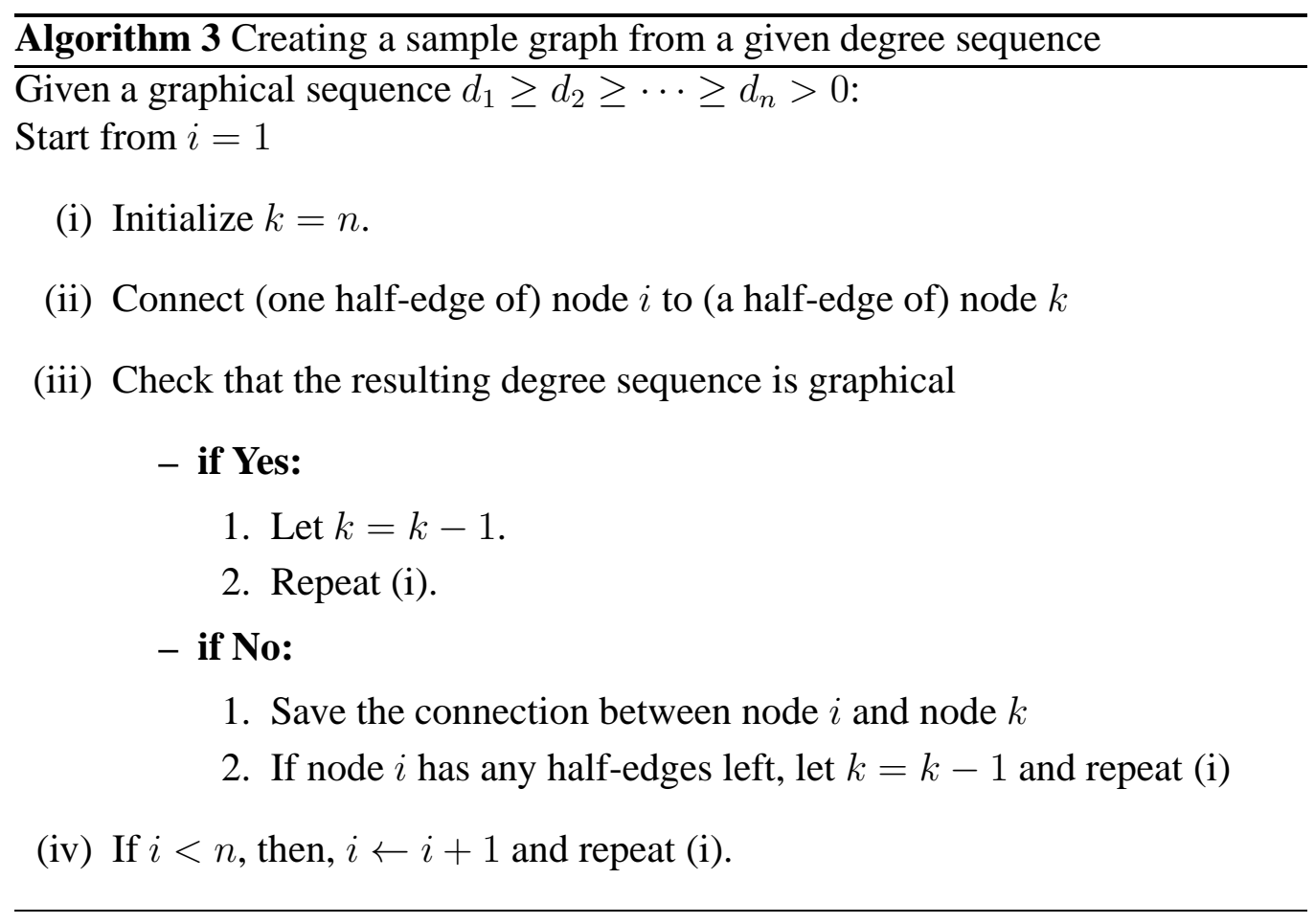

lower degrees. Therefore, Algorithm 3 generates graphs where high-degree nodes tend to connect to low-degree nodes; the resulting graph has assortative property [143, 202]. To overcome this problem, one way is to perform edge swapping repeatedly such that the final graph looses its assortative property. In the edge swapping method, two edges (for example $(1,2)$ and $(3,4)$ ) can be swapped (to $(1,4)$ and $(2,3))$ without changing the degree sequence. Edge swapping method is also used to generate all samples from a given degree sequence; one sample is generated via Algorithm 3 and then, by use of Markov chain Monte-Carlo algorithm based on edge swapping [242], other samples from the graphical realizations of the degree sequence are obtained.

In [143] a swap-free algorithm is proposed to generate all graphical realizations of a true degree sequence. Before proceeding to Algorithm 4 , we first provide definitions which will be used in this algorithm.

Definition 3.4. Let $\mathbf{d}=\left\{d_{1}, \cdots, d_{n}\right\}$ be a degree sequence of a simple graph and $N(i)$ be the set of adjacent nodes of node $i$. Then, the degree sequence reduced by $N(i)$ is denoted by $\left.\mathbf{d}\right|_{N(i)}=\left\{\left.d_{1}\right|_{N(i)}, \cdots,\left.d_{n}\right|_{N(i)}\right\}$ with elements defined as 
follows

$$
\left.d_{k}\right|_{N(i)}= \begin{cases}d_{k}-1, & \text { if } k \in N(i) \\ 0, & \text { if } k=i \\ d_{k}, & \text { otherwise }\end{cases}
$$

Definition 3.5. Let $\left(a_{1}, a_{2}, \ldots, a_{n}\right)$ and $\left(b_{1}, b_{2}, \ldots, b_{n}\right)$ be two sequences. Then, $\left(a_{1}, a_{2}, \ldots, a_{n}\right)<_{C R}\left(b_{1}, b_{2}, \ldots, b_{n}\right)$ if and only if there exists an index $m$ such that $1 \leq m \leq n$ and $a_{m}<b_{m}$ and $a_{i}=b_{i}$ for all $m<i \leq n$.

Let $\mathbf{d}$ be a non-increasing graphical degree sequence. In order to construct the graph, we need to find all possible neighbors $N(i)$ ("allowed set") of each node $i$ such that if we connect this node to its allowed set, then the resulting reduced degree sequence $\left.\mathbf{d}\right|_{N(i)}$ is also graphical, i.e., the graphicality is preserved. Algorithm 4 provides a systematic way (swap-free) to generate all graphical realizations of a true degree sequence (by means of finding all possible neighbors of each node).

\subsection{Numerical Examples}

In this section, numerical examples are given to illustrate the results from $\$ 3.3$ and $\$ 3.5$. The main conclusions are:

1. The infinite duplication-deletion random graph without Markovian dynamics generated by Algorithm 2 satisfies a power law as stated in Theorem 3.4.1. This is illustrated in Example 3.2 below.

2. The degree distribution of the fixed size duplication-deletion random graph generated by Algorithm 2 can be computed from Theorem 3.3.1. This is shown in Example 3.3 below.

3. The estimates obtained by stochastic approximation algorithm (3.14) follow the expected probability distribution precisely without information about the Markovian dynamics. This is illustrated in Example 3.4 below.

Example 3.2. Consider an infinite duplication-deletion random graph without Markovian dynamics generated by Algorithm 2 with $p=0.5$ and $q=0.1$. Theorem 3.4.1 implies that the degree sequence of the resulting graph satisfies a power law with exponent computed using (3.11). Figure 3.3 shows the number of nodes 
Algorithm 4 Constructing all graphs from a graphical degree sequence [143] Given a graphical sequence $d_{1} \geq d_{2} \geq \cdots \geq d_{n}>0$

Start from $i=1$

Step 1: Find neighbors with highes index of node $i$

The aim is to find $A_{R}(i)$ :

(i) Initialize $k=n$.

(ii) Connect node $i$ to node $k$

(iii) Check that the resulting degree sequence is graphical

- if Yes:

1. Let $k=k-1$

2. Repeat (i).

\section{- if No:}

1. Save the connection between node $i$ and node $k$

2. If node $i$ has any stubs left, let $k=k-1$ and repeat (i)

\section{Step 2: Find all possible neighbors of node $i$}

With $<_{C R}$ defined in (3.5), the aim is to find

$\mathcal{A}(i)=\left\{N(i)=\left\{v_{1}, \cdots, v_{d_{i}}\right\} ; N(i)<_{C R} A_{R}(i)\right.$ and $\left.\mathbf{d}\right|_{N(i)}$ is graphical $\}$

(i) Find all sets of nodes who are colexicographically smaller than $A_{R}(1)$ (prospective neighbor sets).

(ii) Connect node $i$ to those neighbors and check if the resulting degree sequence is graphical.

Step 3: For every $N(i) \in \mathcal{A}(i)$ :

- Connect node $i$ to $N(i)$

- Discard node $i$ and compute the reduced degree sequence $\left.\mathbf{d}\right|_{N(i)}$

- Create all graphs from degree sequence $\left.\mathbf{d}\right|_{N(i)}$ using this algorithm 


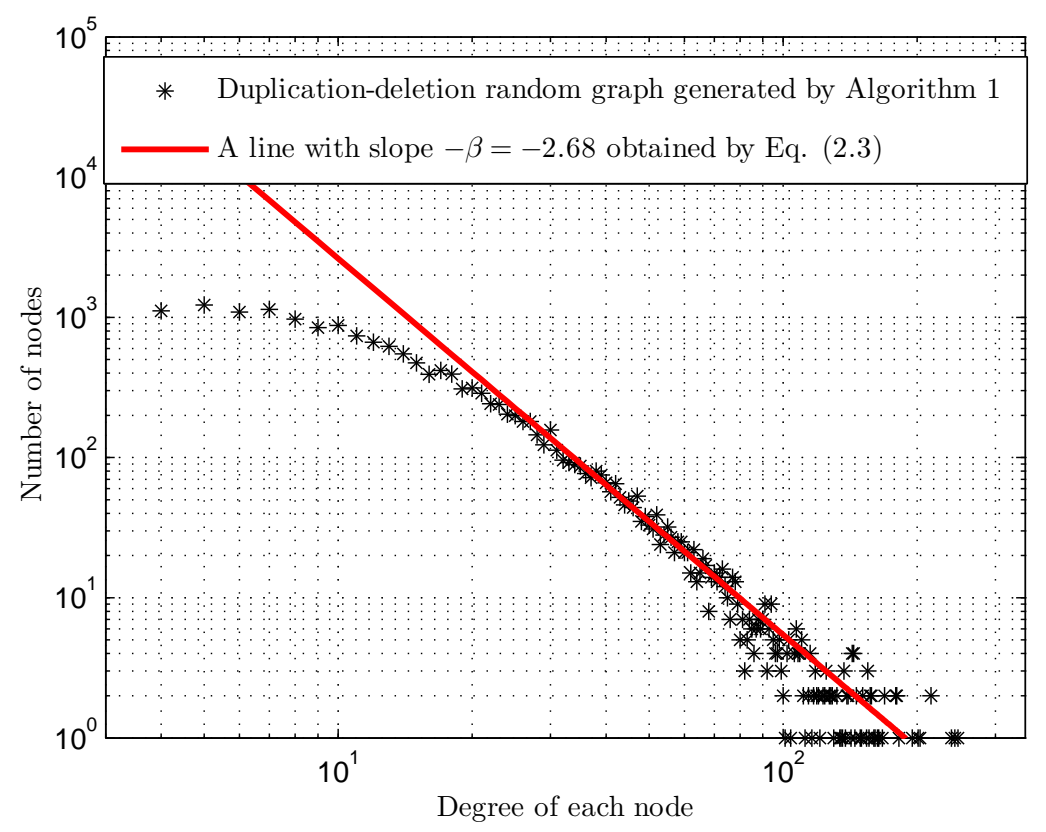

Figure 3.3: The degree distribution of the duplication-deletion random graph satisfies a power law. The parameters are specified in Example 3.2 of $\$ 3.7$.

with specific degree on a logarithmic scale for both horizontal and vertical axes. It can be inferred from the linearity in Figure 3.3 (excluding the nodes with very small degree), that the resulting graph from duplication-deletion process satisfies a power law. As can be seen in Figure 3.3. the power law is a better approximation for the middle points compared to the both ends.

Example 3.3. Consider the fixed size duplication-deletion random graph obtained by Algorithm 2 with $r=0, N_{0}=10, p=0.4$, and $q=0.1$. (We consider no Markovian dynamics here to illustrate Theorem 3.3.1) Figure 3.5 depicts the degree distribution of the fixed size duplication-deletion random graph obtained by Theorem 3.3.1. As can be seen in Figure 3.5 the computed degree distribution is close to that obtained by simulation.

Example 3.4. Consider the fixed size Markov-modulated duplication-deletion random graph generated by Algorithm 2 with $r=0$ and $N_{0}=500$. Assume that the underlying Markov chain has three states, $M=3$. We choose the following values for probabilities of connection and deletion: state (1): $p=q=0.05$, state 


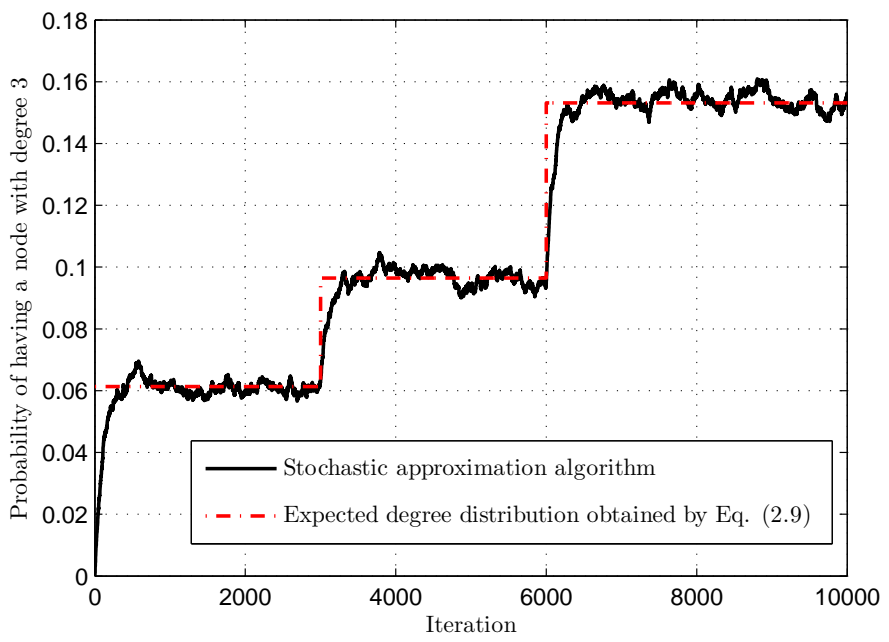

Figure 3.4: The estimated degree distribution obtained by the stochastic approximation algorithm (3.14) and the expected degree distribution computed from (3.9).

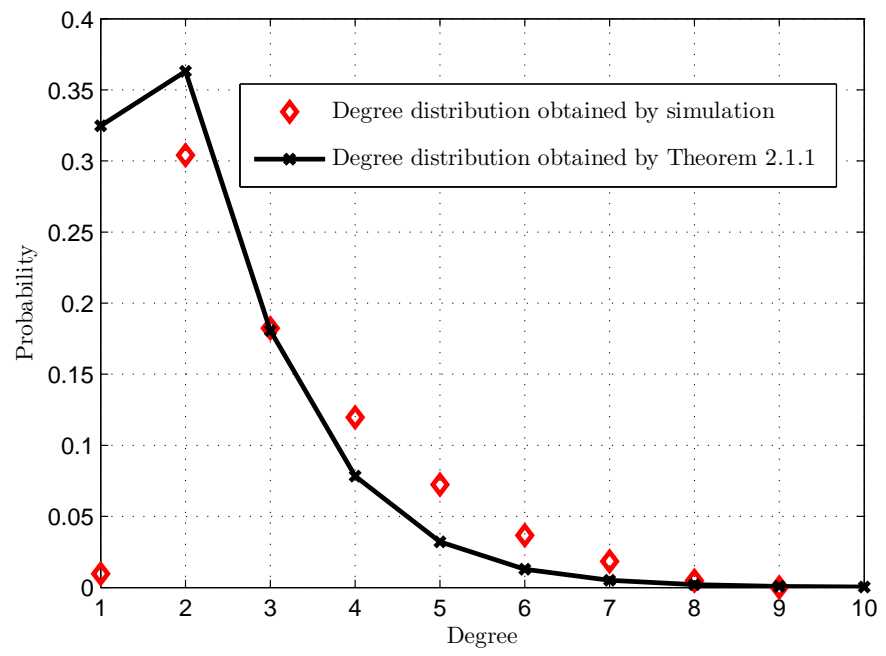

Figure 3.5: The degree distribution of the fixed size Markov-modulated duplication-deletion random graph. The parameters are specified in Example 3.3 of $\$ 3.7$ 
(2): $p=0.2$ and $q=0.1$, and state (3): $p=0.4, q=0.15$. The sample path of the Markov chain jumps at time $n=3000$ from state (1) to state (2) and at time $n=6000$ from state (2) to state (3). As the state of the Markov chain changes, the expected degree distribution, $\bar{g}(\theta)$, obtained by (3.9) evolves over time. Only one element of the expected degree distribution vector is shown in Figure 3.4 via a dotted line. The estimated probability mass function, $\widehat{g}_{n}$, obtained by the stochastic approximation algorithm (3.14) is plotted in Figure 3.4 using a solid line. The figure shows that the estimates obtained by the stochastic approximation algorithm (3.14) follow the expected degree distribution obtained by (3.9) precisely without any information about the Markovian dynamics.

\subsection{Closing Remarks}

\section{Summary}

The interaction between nodes in dynamic social networks is not always fixed and may evolve over time. An example of such time-varying dynamics is the seasonal variations in friendship among college students. The Markov-modulated random graph generated by Algorithm 2 mimics such networks where the dynamics (the connection/deletion probabilities $p, q$ ) depend on the state of nature and evolve over time. Algorithm 2 models these time variations as a finite state Markov chain $\left\{\theta_{n}\right\}$. This model forms our basis to analyze social networks.

We analyzed Markov-modulated duplication-deletion random graphs in terms of degree distribution. When the size of graph is fixed $(r=0)$ and $\rho$ is small, the expected degree distribution of the Markov-modulated duplication-deletion random graph can be uniquely computed from (3.6) for each state of the underlying Markov chain. This result allows us to express the structure of network (degree distribution) in terms of the dynamics of the model.

We also showed that, when the size of the graph is fixed and there is no Markovian dynamics $(M=1, r=1)$, the random graph generated according to Algorithm 2 satisfies a power law with exponent computed from (3.11). The importance of this result is that a single parameter (power law exponent) characterizes the structure of a possibly very large dynamic network.

We further used a stochastic approximation algorithm to estimate the empirical degree distribution of random graphs. The stochastic approximation algorithm (3.14) does not assume any knowledge of the Markov-modulated dynamics of the graph (state of nature). Since the expected degree distribution can be 
uniquely computed, a social sensor can be designed based on (3.14) to track the state of nature using the noisy observations of nodes' degrees. Theorem 3.5.1 showed that the tracking error of the stochastic approximation algorithm is small and is in order of $O(\varepsilon)$.

Finally, a discussion on graph construction was provided for simulation purposes.

\section{Extensions}

Here, we discuss some possible extensions of the framework used in this chapter and avenues for future research which are relevant to social sensors.

In addition to degree distribution, there are some other measures that characterize large networks such as diameter, average path length, clustering, and centrality [132]; for example, [253] uses degree distribution and clustering coefficient to classify social networks. An extension to this work is to use stochastic approximation algorithms and graph theoretic tools employed in this chapter for tracking other characteristics of large networks.

In this chapter, we used probabilistic sampling to obtain measurements from degree distribution. That is, some nodes are randomly chosen and enquired about the number of their neighbors. Another extension to this work is to employ Respondent-driven sampling (RDS) [124, 125, 172] as an approach for sampling from hidden networks in the society. To quote [124],

"hidden populations have two characteristics: first, no sampling frame exists so that the size and boundaries of the population are unknown and second, there exist strong privacy concerns, because membership involves stigmatized or illegal behavior, leading individuals to refuse to cooperate or provide unreliable answers to protect their privacy."

An example of hidden populations is the network of active injection drug users. Algorithm 2 mimics these hidden populations as well: A person usually becomes active injection drug user through another active drug user. Then, he expands his network by connecting to other drug users in this population. An extension to this work is to employ RDS9 to record observations about nodes' connectivity in hidden populations in order to track the structure (degree distribution) of such populations.

\footnotetext{
${ }^{9} \mathrm{RDS}$ is described in Chapter 4 of this monograph.
} 


\section{Chapter 4}

\section{Sensing with Information Diffusion in Complex Social Networks}

\subsection{Introduction}

This chapter considers how a behavior diffuses over a social network comprising of a population of interacting agents and how an underlying stochastic state can be estimated based on sampling the population. As described in [184], there is a wide range of social phenomena such as diffusion of technological innovations, cultural fads, and economic conventions [50] where individual decisions are influenced by the decisions of others. A large body of research on social networks has been devoted to the diffusion of information (e.g., ideas, behaviors, trends) [108], and particularly on finding a set of target nodes so as to maximize the spread of a given product [193, 56].

Consider a social network where the states of individual nodes evolve over time as a probabilistic function of the states of their neighbors and an underlying target process. The evolution in the state of agents in the network can be viewed as diffusion of information in the network. Such Susceptible-Infected-Susceptible (SIS) models for diffusion of information in social networks has been extensively studied in [132, 183, 184, 212, 249] to model, for example, the adoption of a new technology in a consumer market.

In this chapter we consider two extensions of the SIS model: First, the states of individual nodes evolve over time as a probabilistic function of the states of their neighbors and an underlying target process. The underlying target process can be viewed as the market conditions or competing technologies that evolve with 
time and affect the information diffusion. Second, the nodes in the social network are sampled randomly to determine their state. (We will review two recent methods for sampling social networks, namely, social sampling and respondent-driven sampling.) As the adoption of the new technology diffuses through the network, its effect is observed via sentiments (such as tweets) of these selected members of the population. These selected nodes act as social sensors. In signal processing terms, the underlying target process can be viewed as a signal, and the social network can be viewed as a sensor. The key difference compared to classical signal processing is that the social network (sensor) has dynamics due to the information diffusion.

\subsubsection{Aim}

Our aim is to estimate the underlying target state and the state probabilities of the nodes by sampling measurements at nodes in the social network. In a Bayesian estimation context, this is equivalent to a filtering problem involving estimation of the state of a prohibitively large scale Markov chain in noise. The key idea is to use mean field dynamics as an approximation (with provable bounds) for the information diffusion and, thereby, obtain a tractable model. Such mean field dynamics] have been studied in [27] and applied to social networks in [183, 184, [249]. For an excellent recent exposition of interacting particle systems comprising of agents each with a finite state space, see [9], where the more apt term "Finite Markov Information Exchange (FMIE) process" is used.

\subsubsection{Motivation}

A typical application of such social sensors arises in the measurement of the adoption of a new product using a micro-blogging platform like Twitter. The adoption of the technology diffuses through the market but its effects can only be observed through the tweets of select individuals of the population. These selected individuals act as sensors for estimating the diffusion. They interact and learn from the decisions (tweeted sentiments) of other members. (This is similar to social learning.) Suppose the state of nature changes suddenly due to a sudden market shock or presence of a new competitor. The goal for a market analyst or product

\footnotetext{
${ }^{1}$ Recently, there has also been substantial interest in mean field games [129]. These are outside the scope of the current monograph.
} 
manufacturer is to estimate the target state so as to detect the market shock or new competitor. This is a Bayesian filtering problem².

To quote from Wikipedia: "Sentiment analysis refers to the use of natural language processing, text analysis and computational linguistics to identify and extract subjective information in source materials." The following excerpt from [28] illustrates the increasing importance of social sentiment with the growth of social networking:

- " $53 \%$ of people on Twitter recommend companies and/or products in their Tweets, with $48 \%$ of them delivering on their intention to buy the product. (ROI Research for Performance, June 2010)

- The average consumer mentions specific brands over 90 times per week in conversations with friends, family, and co-workers. (Keller Fay, WOMMA, 2010)

- Consumer reviews are significantly more trusted-nearly 12 times morethan descriptions that come from manufacturers, according to a survey of US mom Internet users by online video review site EXPO. (eMarketer, February 2010)

- In a study conducted by social networking site myYearbook, $81 \%$ of respondents said they had received advice from friends and followers relating to a product purchase through a social site; $74 \%$ of those who received such advice found it to be influential in their decision. (ClickZ, January 2010)

As another example, [222] considers each Twitter user as a sensor and uses a particle filtering algorithm to estimate the centre of earthquakes and trajectories of typhoons. As pointed out in [222], an important characteristic of microblogging services such as Twitter is that they provide real-time sensing-Twitter users tweet several times a day, whereas standard blog users update information once every several days.

\footnotetext{
${ }^{2} \mathrm{~A}$ more general approach (which we will not elaborate on) is to formulate the problem as a Bayesian quickest detection problem (or more generally a stopping time problem) that seeks to minimize a cost function that involves the sum of the false alarm and decision delay. Bayesian filtering is of course an integral part of such change detection.
} 


\subsubsection{Experimental Studies}

Here, we mention papers that investigate the diffusion of information in real-world social networks such as Facebook, Twitter, and Blogs. Motivated by marketing applications, [237] studies the diffusion (contagion) behaviour in Facebook 3 . Using data on around 260000 Facebook pages 4 , [237] analyzes how information diffuses on Facebook. The topological and temporal characteristics of information diffusion on weblogs are also studied in [176]. Twitter with around 200 million active users and 400 million tweets per day, has become a powerful information sharing tool [168, 256].

\subsection{Social Network Model}

A social network is modelled as a graph with $N$ vertices:

$$
G=(V, E), \text { where } V=\{1,2, \ldots, N\}, \text { and } E \subseteq V \times V .
$$

Here, $V$ denotes the finite set of vertices, and $E$ denotes the set of edges. In social networks, it is customary to use the terminology network, nodes and links for graph, vertices and edges, respectively.

We use the notation $(m, n)$ to refer to a link between node $m$ and $n$. The network may be undirected in which case $(m, n) \in E$ implies $(n, m) \in E$. In undirected graphs, to simplify notation, we use the notation $m, n$ to denote the undirected link between node $n$ and $m$. If the graph is directed, then $(m, n) \in E$ does not imply that $(n, m) \in E$. We will assume that self loops (reflexive links) of the form $i, i$ are excluded from $E$.

An important parameter of a social network $G=(V, E)$ is the connectivity of its nodes. Let $\mathcal{N}^{(m)}$ and $D^{(m)}$ denote the neighbourhood set and degree (or connectivity) of a node $m \in V$, respectively. That is, with $|\cdot|$ denoting cardinality,

$$
\mathcal{N}^{(m)}=\{n \in V: m, n \in E\}, \quad D^{(m)}=\left|\mathcal{N}^{(m)}\right|
$$

For convenience, we assume that the maximum degree of the network is uniformly bounded by some fixed integer $\bar{D}$.

\footnotetext{
${ }^{3}$ In US in 2010, $\$ 1.7$ billion was spent on advertising through social media. The share of Facebook is 53\% in this market [220].

${ }^{4}$ Facebook pages usually advertise products, services, bands, celebrities, etc.
} 
Let $N(d)$ denote the number of nodes with degree $d$, and let the degree distribution $P(d)$ specify the fraction of nodes with degree $d$. That is, for $d=$ $0,1, \ldots, \bar{D}$,

$$
N(d)=\sum_{m \in V} I\left\{D^{(m)}=d\right\}, \quad P(d)=\frac{N(d)}{N} .
$$

Here, $I\{\cdot\}$ denotes the indicator function. Note that $\sum_{d} P(d)=1$. The degree distribution can be viewed as the probability that a node selected randomly with uniform distribution on $V$ has a connectivity $d$.

Random graphs generated to have a degree distribution $P$ that is Poisson were formulated by Erdös and Renyi [83]. Several recent works show that large scale social networks are characterized by connectivity distributions that are different to Poisson distributions. For example, the internet, www have a power law connectivity distribution $P(d) \propto d^{-\gamma}$, where $\gamma$ ranges between 2 and 3. Such scale free networks are studied in [24]. In the rest of this chapter, we assume that the degree distribution of the social network is arbitrary but known-allowing an arbitrary degree distribution facilities modelling complex networks.

\subsubsection{Target Dynamics}

Let $k=0,1, \ldots$ denote discrete time. Assume the target process $s$ is a finite state Markov chain with transition probability

$$
A_{s s^{\prime}}=\mathbb{P}\left(s_{k+1}=s^{\prime} \mid s_{k}=s\right) .
$$

In the example of technology diffusion, the target process can denote the availability of competition or market forces that determine whether a node adopts the technology. In the model below, the target state will affect the probability that an agent adopts the new technology.

\subsubsection{Diffusion of Information in Social Network}

The model we present below for the diffusion of information in the social network is called the Susceptible-Infected-Susceptible (SIS) model [212, 249]. The diffusion of information is modelled by the time evolution of the state of individual nodes in the network. Let $x_{k}^{(m)} \in\{0,1\}$ denote the state at time $k$ of each node $m$ in the social network. Here, $x_{k}^{(m)}=0$ if the agent at time $k$ is susceptible and 
$x_{k}^{(m)}=1$ if the agent is infected. At time $k$, the state vector of the $N$ nodes is

$$
x_{k}=\left[x_{k}^{(1)}, \ldots, x_{k}^{(N)}\right]^{\prime} \in\{0,1\}^{N} .
$$

Assume that the process $x$ evolves as a discrete time Markov process with transition law depending on the target state $s$. If node $m$ has degree $D^{(m)}=d$, then the probability of node $m$ switching from state $i$ to $j$ is

$$
\begin{gathered}
\mathbb{P}\left(x_{k+1}^{(m)}=j \mid x_{k}^{(m)}=i, x_{k}^{(i-)}, s_{k}=s\right) \\
=p_{i j}\left(d, A_{k}^{(m)}, s\right), i, j \in\{0,1\} .
\end{gathered}
$$

Here, $A_{k}^{(m)}$ denotes the number of infected neighbors of node $m$ at time $k$. That is,

$$
A_{k}^{(m)}=\sum_{n \in N^{(m)}} I\left\{n: x_{k}^{(m)}=1\right\} .
$$

In words, the transition probability of an agent depends on its degree distribution and the number of active neighbors.

With the above probabilistic model, we are interested in modelling the evolution of infected agents over time. Let $\rho_{k}(d)$ denote the fraction of infected nodes at each time $k$ with degree $d$. We call $\rho$ as the infected node distribution. So

$$
\rho_{k}(d)=\frac{1}{N(d)} \sum_{m \in V} I\left\{D^{(m)}=d, x_{k}^{(m)}=1\right\}, \quad d=0,1, \ldots, \bar{D}
$$

We assume that the infection spreads according to the following dynamics:

1. At each time instant $k$, a single agent, denoted by $m$, amongst the $N$ agents is chosen uniformly. Therefore, the probability that the chosen agent $m$ is infected and of degree $d$ is $\rho_{k}(d) P(d)$. The probability that the chosen agent $m$ is susceptible and of degree $d$ is $\left(1-\rho_{k}(d)\right) P(d)$.

2. Depending on whether its state $x_{k}^{(m)}$ is infected or susceptible, the state of agent $m$ evolves according to the transition probabilities specified in (4.5).

With the Markov chain transition dynamics of individual agents specified above, it is clear that the infected distribution $\rho_{k}=\left(\rho_{k}(1), \ldots, \rho_{k}(\bar{D})\right)$ is an $\prod_{d=1}^{\bar{D}} N(d)$ state Markov chain. Indeed, given $\rho_{k}(d)$, due to the infection dynamics specified above

$$
\rho_{k+1}(d) \in\left\{\rho_{k}(d)-\frac{1}{N(d)}, \rho_{k}(d)+\frac{1}{N(d)}\right\} .
$$


Our aim below is to specify the transition probabilities of the Markov chain $\rho$. Let us start with the following statistic that forms a convenient parametrization of the transition probabilities. Given the infected node distribution $\rho_{k}$ at time $k$, define $\theta\left(\rho_{k}\right)$ as the probability that at time $k$ a uniformly sampled link in the network points to an infected node. We call $\theta\left(\rho_{k}\right)$ as the infected link probability. Clearly

$$
\begin{aligned}
\theta\left(\rho_{k}\right) & =\frac{\sum_{d=1}^{\bar{D}}(\# \text { of links from infected node of degree } d)}{\sum_{d=1}^{\bar{D}}(\# \text { of links of degree } d)} \\
& =\frac{\sum_{d=1}^{\bar{D}} d P(d) \rho_{k}(d)}{\sum_{d}^{\bar{D}} d P(d)}
\end{aligned}
$$

In terms of the infected link probability $\theta$, we can now specify the scaled transition probabilities 5 of the process $\rho$ :

$$
\begin{aligned}
& \bar{p}_{01}\left(d, \theta_{k}, s\right) \stackrel{\text { defn }}{=} \frac{1}{P(d)} \mathbb{P}\left(\rho_{k+1}(d)=\rho_{k}(d)+\frac{1}{N(d)} \mid s_{k}=s\right) \\
&=\left(1-\rho_{k}(d)\right) \sum_{a=0}^{d} p_{01}(d, a, s) \mathbb{P}(a \text { out of } l \text { neighbors infected }) \\
&=\left(1-\rho_{k}(d)\right) \sum_{a=0}^{d} p_{01}(d, a, s)\left(\begin{array}{l}
d \\
a
\end{array}\right) \theta_{k}^{a}\left(1-\theta_{k}\right)^{d-a} \\
& \bar{p}_{10}\left(d, \theta_{k}, s\right) \stackrel{\text { defn }}{=} \frac{1}{P(d)} \mathbb{P}\left(\rho_{k+1}(d)=\rho_{k}(d)-\frac{1}{N(d)} \mid s_{k}=s\right) \\
&=\rho_{k}(d) \sum_{a=0}^{d} p_{10}(d, a, s)\left(\begin{array}{l}
d \\
a
\end{array}\right) \theta_{k}^{a}\left(1-\theta_{k}\right)^{d-a}
\end{aligned}
$$

In the above, the notation $\theta_{k}$ is the short form for $\theta\left(\rho_{k}\right)$. The transition probabilities $\bar{p}_{01}$ and $\bar{p}_{10}$ defined above model the diffusion of information about the target state $s$ over the social network. We have the following martingale representation theorem for the evolution of Markov process $\rho$.

Let $\mathcal{F}_{k}$ denote the sigma algebra generated by $\left\{\rho_{0}, \ldots, \rho_{k}, s_{0}, \ldots s_{k}\right\}$.

\footnotetext{
${ }^{5}$ The transition probabilities are scaled by the degree distribution $P(d)$ for notational convenience. Indeed, since $N(d)=N P(d)$, by using these scaled probabilities we can express the dynamics of the process $\rho$ in terms of the same-step size $1 / N$ as described in Theorem 4.2.1. Throughout this chapter, we assume that the degree distribution $P(d), d \in\{1,2, \ldots, \bar{D}\}$, is uniformly bounded away from zero. That is, $\min _{d} P(d)>\epsilon$ for some positive constant $\epsilon$.
} 
Theorem 4.2.1. For $d=1,2, \ldots, \bar{D}$, the infected distributions evolve as

$$
\rho_{k+1}(d)=\rho_{k}(d)+\frac{1}{N}\left[\bar{p}_{01}\left(d, \theta\left(\rho_{k}\right), s_{k}\right)-\bar{p}_{10}\left(d, \theta\left(\rho_{k}\right), s_{k}\right)+w_{k+1}\right]
$$

where $w$ is a martingale increment process, that is $\mathbf{E}\left\{w_{k+1} \mid \mathcal{F}_{k}\right\}=0$. Recall $s$ is the finite state Markov chain that models the target process.

The above theorem is a well-known martingale representation of a Markov chain [78] - it says that a discrete time Markov process can be obtained by discrete time filtering of a martingale increment process. The theorem implies that the infected distribution dynamics resemble what is commonly called a stochastic approximation (adaptive filtering) algorithm in statistical signal processing: the new estimate is the old estimate plus a noisy update (the "noise" being a martingale increment) that is weighed by a small step size $1 / N$ when $N$ is large. Subsequently, we will exploit the structure in Theorem 4.2.1 to devise a mean field dynamics model which has a state of dimension $\bar{D}$. This is to be compared with the intractable state dimension $\prod_{d=1}^{\bar{D}} N(d)$ of the Markov chain $\rho$.

Remark 4.1 (Data Incest). In the above susceptible-infected-susceptible (SIS) model, the following sequence of events that is similar to a data incest event (discussed in 92.3 ) is possible: Node $m$ infects its neighbor node $n$; then, node $m$ switches in state from infected to susceptible; then, node $n$ re-infects node $m$. Therefore, misinformation can propagate in the network according to such loops. An alternative model is the susceptible-infected-recovered (SIR) model where each node has three states: susceptible, infected, and recovered. If the recovered state is made absorbing, then such incest events can be eliminated.

Example 4.1. We discuss examples of transition probabilities $p_{i j}\left(d, A_{k}^{(m)}, s\right)$ defined in (4.5) for information diffusion. These examples are provided in [183] and deal with how the use of a new technology/product spreads in a social network. Let the state of agent $m$ be $x_{k}^{(m)}=0$ if the agent at time $k$ has not adopted the new technology, and $x_{k}^{(m)}=1$ if the agent has adopted the new technology. As mentioned earlier, the target state $s_{k}$ determines the availability of a competing technology/product or, alternatively, the available market for the product.

In deciding whether to adopt the new technology, each agent $m$ considers the following costs and rewards: 
1. The cost node $m$ pays for adopting the new technology is $c^{(m)}$. Assume that, at time $k$, the costs $c^{(m)}, m=1, \ldots, N$ are independent and identically distributed random variables with cumulative distribution function $P_{c, s_{k}}$. The fact that this distribution depends on the target state $s_{k}$ (competing technology that evolves as a Markov chain) permits correlation of the costs $c$ over time.

2. If an agent $m$ has $A_{k}^{(m)}$ neighbors at time $k$, then it obtains a benefit of $r A_{k}^{(m)}$ for adopting the technology, where $r$ is a positive real number.

The agent is a myopic optimizer and, hence, chooses to adopt the technology only if $c^{(m)}>r A_{k}^{(m)}$. Therefore, the transition probabilities in (4.5) are

$$
p_{01}\left(d, A_{k}^{(m)}, s_{k}\right)=\mathbb{P}\left(c^{(m)}>r A_{k}^{(m)}\right)=1-P_{c, s_{k}}\left(r A_{k}^{(m)}\right) .
$$

Assume that if the product deteriorates/fails, the agent can no longer use the product (and will then need to reconsider the possibility of adopting it). If the product fails with probability $p_{F}$, then

$$
p_{10}\left(d, A_{k}^{(m)}, s_{k}\right)=p_{F} .
$$

Notice that the transition probabilities in (4.13) do not depend on the node's connectivity $d$. Constructing cases where the transition probabilities depend on $d$ is straightforward. For example, suppose a node picks a single neighbor uniformly from its $d$ neighbors and then receives a benefit of $r$ if this randomly chosen neighbour has adopted the product. The probability of choosing an active neighbor from $A_{k}^{(m)}$ active neighbors, given a total of $d$ neighbors, is clearly $A_{k}^{(m)} / d$. Then, assuming the agent acts as a myopic optimizer, it will adopt the product if $\mathbb{P}\left(c^{(m)}>r A_{k}^{(m)} / d\right)$. Therefore,

$$
p_{01}\left(d, A_{k}^{(m)}, s_{k}\right)=1-P_{c, s_{k}}\left(\frac{r A_{k}^{(m)}}{d}\right) .
$$

\subsection{Sentiment-Based Sensing Mechanism}

We now describe the sensing mechanism used to measure the active link probability distribution $\rho_{k}$ and target state in the above social network. 
If node $m$ has state $x_{k}^{(m)}=x$ at time $k$, and the target state is $s_{k}=s$, then node $m$ communicates with message $y_{k}^{(m)}$ where

$$
y_{k}^{(m)} \sim B_{x y}, \quad \text { where } \quad y \in\{0,1, \ldots, Y\} .
$$

That is, the message $y_{k}$ is generated according to the conditional probabilities $B_{x y}=\mathbb{P}\left(y_{k}^{(m)}=y \mid x_{k}^{(m)}=x\right)$. These elements correspond to the number of tweets or sentiment of node $m$ about the product based on its current state. It is assumed that $y_{k}^{(m)}$ is statistically independent of $y_{k}^{(n)}, n \neq m$.

An important question regarding sensing in a social network is: How can one construct a small but representative sample of a social network with a large number of nodes? Leskovec \& Faloutsos in [175] study and compare several scaledown and back-in-time sampling procedures. The simplest possible sampling scheme for a population is uniform sampling. We also briefly describe social sampling and respondent-driven sampling which are recent methods that have become increasingly popular.

\subsubsection{Uniform Sampling}

Consider the following sampling-based measurement strategy. At each period $k$, $\alpha(d)$ individuals are sampled $\sqrt{6}$ independently and uniformly from the population $N(d)$ comprising of agents with connectivity degree $d$. That is, a uniform distributed i.i.d. sequence of nodes, denoted by $\left\{m_{l}, l=1: \alpha(d)\right\}$, is generated from the population $N(d)$. The messages $y_{k}^{\left(m_{l}\right)}$ of these $\alpha(d)$ individuals are recorded. From these independent samples, the empirical sentiment distribution $z_{k}(d)$ of degree $d$ nodes at each time $k$ is obtained as

$$
z_{k}(d, y)=\frac{1}{\alpha(d)} \sum_{l=1}^{\alpha(d)} I\left\{y_{k}^{\left(m_{l}\right)}=y\right\}, \quad y=1, \ldots, Y .
$$

At each time $k$, the empirical sentiment distribution $z_{k}$ can be viewed as noisy observations of the infected distribution $\rho_{k}$ and target state process $s_{k}$.

\subsubsection{Non-Uniform Social Sampling}

Social sampling is an extensive area of research; see [66] for recent results. In social sampling, participants in a poll respond with a summary of their friend's

\footnotetext{
${ }^{6}$ For large population sizes $N$, sampling with and without replacement are equivalent.
} 
responses. This leads to a reduction in the number of samples required. If the average degree of nodes in the network is $d$, then the savings in the number of samples is by a factor of $d$, since a randomly chosen node summarizes the results form $d$ of its friends. However, the variance and bias of the estimate depend strongly on the social network structure?. In [66], a social sampling method is introduced and analyzed where nodes of degree $d$ are sampled with probability proportional to $1 / d$. This is intuitive since weighing neighbors' values by the reciprocal of the degree undoes the bias introduced by large degree nodes. It then illustrates this social sampling method and variants on the LIVEJOURNAL network (livejournal.com) comprising of more than 5 million nodes and 160 million directed edges.

\subsubsection{MCMC Based Respondent-Driven Sampling (RDS)}

Respondent-driven sampling (RDS) was introduced by Heckathorn [124, 125, 172] as an approach for sampling from hidden populations in social networks and has gained enormous popularity in recent years. There are more than 120 RDS studies worldwide involving sex workers and injection drug users [190]. As mentioned in [106], the U.S. Centers for Disease Control and Prevention (CDC) recently selected RDS for a 25-city study of injection drug users that is part of the National HIV Behavioral Surveillance System [170].

RDS is a variant of the well known method of snowball sampling where current sample members recruit future sample members. The RDS procedure is as follows: A small number of people in the target population serve as seeds. After participating in the study, the seeds recruit other people they know through the social network in the target population. The sampling continues according to this procedure with current sample members recruiting the next wave of sample members until the desired sampling size is reached. Typically, monetary compensations are provided for participating in the data collection and recruitment.

RDS can be viewed as a form of Markov Chain Monte Carlo (MCMC) sampling (see [106] for an excellent exposition). Let $\left\{m_{l}, l=1: \alpha(d)\right\}$ be the realization of an aperiodic irreducible Markov chain with state space $N(d)$ comprising of nodes of degree $d$. This Markov chain models the individuals of degree

\footnotetext{
${ }^{7}$ In [66], a nice intuition is provided in terms of intent polling and expectation polling. In intent polling, individual are sampled and asked who they intend to vote for. In expectation polling, individuals are sampled and asked who they think would win the election. For a given sample size, one would believe that expectation poling is more accurate than intent polling since in expectation polling, an individual would typically consider its own intent together with the intents of its friends.
} 
$d$ that are snowball sampled, namely, the first individual $m_{1}$ is sampled and then recruits the second individual $m_{2}$ to be sampled, who then recruits $m_{3}$ and so on. Instead of the independent sample estimator (4.17), an asymptotically unbiased MCMC estimate is then generated as

$$
\frac{\sum_{l=1}^{\alpha(d)} \frac{I\left(y_{k}^{\left(m_{l}\right)}=y\right)}{\pi\left(m_{l}\right)}}{\sum_{l=1}^{\alpha(d)} \frac{1}{\pi\left(m_{l}\right)}}
$$

where $\pi(m), m \in N(d)$, denotes the stationary distribution of the Markov chain. For example, a reversible Markov chain with prescribed stationary distribution is straightforwardly generated by the Metropolis Hastings algorithm.

In RDS, the transition matrix and, hence, the stationary distribution $\pi$ in the estimator (4.18) is specified as follows: Assume that edges between any two nodes $m$ and $n$ have symmetric weights $W(m, n)$ (i.e., $W(m, n)=W(n, m)$, equivalently, the network is undirected). In RDS, node $m$ recruits node $n$ with transition probability $W(m, n) / \sum_{n} W(m, n)$. Then, it can be easily seen that the stationary distribution is $\pi(m)=\sum_{n \in V} W(m, n) / \sum_{m \in V, n \in V} W(m, n)$. Using this stationary distribution, along with the above transition probabilities for sampling agents in (4.18), yields the RDS algorithm.

It is well known that a Markov chain over a non-bipartite connected undirected network $G$ is aperiodic. Then, the initial seed for the RDS algorithm can be picked arbitrarily, and the above estimator is an asymptotically unbiased estimator.

Note the difference between RDS and social sampling: RDS uses the network to recruit the next respondent, whereas social sampling seeks to reduce the number of samples by using people's knowledge of their friends' (neighbors') opinions.

\subsubsection{Extrinsic Observations}

In addition to the above sentiment based observation process, often extrinsic measurements of the target state are available. For example, if the target state denotes the price/availability of products offered by competitors or the current market, then economic indicators yield noisy measurements. Let $o_{k}$ denote noisy measurements of the target state $s_{k}$ at time $k$. These observations are obtained as

$$
o_{k} \sim \bar{B}_{s o} \stackrel{\text { defn }}{=} \mathbb{P}\left(o_{k}=o \mid s_{k}=s\right), \text { where } o \in\{1,2, \ldots, O\}
$$

Remark 4.2. The reader may be familiar with the DARPA network challenge in 2009 where the locations of 10 red balloons in the continental US were to be 
determined using social networking. In this case, the winning MIT Red Balloon Challenge Team used a recruitment based sampling method. The strategy can also be viewed as a variant of the Query Incentive Network model of [146].

\subsection{Mean Field Dynamics for Social Sensing}

The aim here is to construct statistical signal processing algorithms for computing the minimum mean square error estimate of the infected distribution $\rho_{k}$ and target state $s_{k}$ given the sequence of sentiment observations $z_{1: k}=\left\{z_{1}, \ldots, z_{k}\right\}$. This problem is intractable due to the dimension $\prod_{d=1}^{\bar{D}} N(d)$ of the state $\rho_{k}$ when the number of agents $N$ is large. Fortunately, the mean field dynamics approximation of the information diffusion has a state dimension of $\bar{D}$ and will be shown to be an excellent approximation as the number of agents $N$ becomes large. This is the subject of the current section.

Depending on the time scale on which the target state $s$ evolves, there are two possible asymptotic limits for the mean field dynamics for information diffusion in the social network:

1. Diffusion matched to target dynamics;

2. Diffusion faster than target dynamics.

We consider both these cases below and formulate estimation of the states $\rho_{k}, s_{k}$, given the sequence of sentiment observations $z_{1: k}$, as a nonlinear filtering problem. The main consequence is that the resulting mean field dynamics filtering problem can be solved straightforwardly via the use of sequential Markov chain Monte-Carlo methods. This is in contrast to the original filtering problem which is intractable as $N \rightarrow \infty$.

\subsection{Mean Field Dynamics: Information Diffusion Matched to Target State Dynamics}

The main assumption here is that the diffusion of information flow in the social network, namely $\rho$, evolves on the same time-scale as the target state $s$. This is made explicit in the following assumption. 
Assumption 4.1. The target state $s_{k}$ has transition probability matrix $A=I+$ $\frac{1}{N} Q$, where $Q$ is a generator matrix, i.e., $q_{i j} \geq 0$ for $i \neq j$ and $Q \mathbf{1}=\mathbf{0}$, where $\mathbf{1}$ and $\mathbf{0}$ are column vectors of ones and zeros, respectively.

\subsubsection{Mean Field Dynamics}

The mean field dynamics state that as the number of agents $N$ grows to infinity, the dynamics of the infected distribution $\rho$, described by (4.12), in the social network evolves according to an ordinary differential equation that is modulated by a continuous-time Markov chain which depends on the target state evolution $s$. More specifically, under Assumption 4.1, the mean field dynamics for the system (4.12) are as follows: For $d=1,2, \ldots, \bar{D}$,

$$
\begin{aligned}
\frac{d \rho_{t}(d)}{d t} & =\bar{p}_{01}\left(d, \theta\left(\rho_{t}\right), s_{t}\right)-\bar{p}_{10}\left(d, \theta\left(\rho_{t}\right), s_{t}\right) \\
\bar{p}_{01}\left(d, \theta, s_{t}\right) & =\left(1-\rho_{t}(d)\right) \sum_{a=0}^{d} p_{01}\left(d, a, s_{t}\right)\left(\begin{array}{l}
d \\
a
\end{array}\right) \theta^{a}(1-\theta)^{d-a} \\
\bar{p}_{10}\left(d, \theta, s_{t}\right) & =\rho_{t}(d) \sum_{a=0}^{d} p_{10}\left(d, a, s_{t}\right)\left(\begin{array}{l}
d \\
a
\end{array}\right) \theta^{a}(1-\theta)^{d-a} \\
\theta\left(\rho_{t}\right) & =\frac{\sum_{d=1}^{\bar{D}} d P(d) \rho_{t}(d)}{\sum_{d}^{\bar{D}} d P(d)}
\end{aligned}
$$

where $s_{t}$ is a continuous time Markov chain with generator $Q$.

That the above mean field dynamics follow from (4.12) is intuitive. Such averaging results are well known in the adaptive filtering community where they are deployed to analyze the convergence of adaptive filters. The difference here is that the limit mean field dynamics are not deterministic but Markov modulated. Moreover, the mean field dynamics here constitute a model for information diffusion, rather than the asymptotic behavior of an adaptive filtering algorithm. As mentioned earlier, from an engineering point of view, the mean field dynamics yield a tractable model for estimation.

To formalize the convergence of the discrete time dynamics (4.12) of the infected distribution to the mean field dynamics (4.20) as the number of agents $N \rightarrow \infty$, we first need to re-write the discrete time process $\rho_{k}$ in (4.12) as a continuous time process. This is straightforwardly done by constructing a piecewise linear interpolation between the discrete-time points-in classical signal processing, this constitutes what is commonly called a first-order hold interpolation. 
Define the sampling period as $1 / N$ and then define the continuous time linear interpolated process 8 associated with $\rho_{k}$ as

$$
\widetilde{\rho}_{t}^{N}(d)=\rho_{k}(d)+\frac{t-k / N}{1 / N}\left(\rho_{k+1}(d)-\rho_{k}(d)\right)
$$

for continuous time $t \in\left[\frac{k}{N}, \frac{(k+1)}{N}\right), k=0,1, \ldots$.

We then have the following exponential bound result for the error $\left\|\widetilde{\rho}_{t}^{N}-\rho_{t}\right\|_{\infty}$; recall $\rho_{t}$ is given the mean field dynamics (4.20) while the actual system's infected distribution is $\widetilde{\rho}_{t}^{N}$ :

Theorem 4.5.1. Suppose Assumption 4.1 holds. Then, for $N$ sufficiently large,

$$
\mathbb{P}\left\{\max _{0 \leq t \leq T}\left\|\widetilde{\rho}_{t}^{N}-\rho_{t}\right\|_{\infty} \geq \epsilon\right\} \leq C_{1} \exp \left(-C_{2} \epsilon^{2} N\right)
$$

where $C_{1}$ and $C_{2}$ are positive constants and $T$ is any finite time horizon.

The proof of the above theorem follows from [27, Lemma 1]. The exponential bound follows from an application of the Azuma-Hoeffding inequality. The above theorem provides an exponential bound (in terms of the number of agents $N$ ) for the probability of deviation of the sample path of the infected distribution from the mean field dynamics for any finite time interval $T$.

Remark: For the signal processing reader more familiar with discrete-time averaging theory, given the discrete time system (4.12), the discrete-time mean field dynamics are:

$$
\bar{\rho}_{k+1}(d)=\bar{\rho}_{k}(d)+\frac{1}{N}\left[\bar{p}_{01}\left(d, \theta\left(\bar{\rho}_{k}\right), s_{k}\right)-\bar{p}_{10}\left(d, \theta\left(\bar{\rho}_{k}\right), s_{k}\right)\right] .
$$

Then the following is the discrete time equivalent of Theorem 4.5.1; For a discrete time horizon of $T$ points, the deviation between the mean field dynamics $\bar{\rho}_{k}$ in (4.23) and actual infected distribution in $\rho_{k}$ (4.12) satisfies

$$
\mathbb{P}\left\{\max _{0 \leq k \leq T}\left\|\bar{\rho}_{k}-\rho_{k}\right\|_{\infty} \geq \epsilon\right\} \leq C_{1} \exp \left(-C_{2} \epsilon^{2} N\right)
$$

providing $T=O(N)$.

\footnotetext{
${ }^{8}$ One can also consider a piecewise constant interpolation as widely used in [167] for weak convergence analysis of stochastic approximation algorithms; then, the appropriate function space is the space of Càdlàg functions, which is usually denoted $D[0, T]$.
} 


\section{Some Perspective}

The stochastic approximation and adaptive filtering literature [29, 167] has several averaging analysis methods for recursions of the form (4.12). The well studied mean square error analysis [29, 167] computes bounds on $\mathbf{E}\left\|\widetilde{\rho}_{t}^{N}-\rho_{t}\right\|^{2}$ instead of the maximum deviation in Theorem 4.5.1. A mean square error analysis of estimating a Markov modulated empirical distribution is given in [260]. Such mean square analysis assume a finite but small step size $1 / N$ in (4.12). Another powerful class of analysis methods involves weak convergence. These seek to prove that $\lim _{N \rightarrow \infty} \mathbb{P}\left\{\sup _{t \leq T}\left\|\widetilde{\rho}_{t}^{N}-\rho_{t}\right\| \geq \epsilon\right\}=0$. Weak convergence methods are powerful enough to tackle Markovian noise, whereas in Theorem 4.5.1 the noise term is a much simpler martingale increment.

On the other hand, unlike weak convergence results, Theorem 4.5.1 offers an exponential bound. This is useful in the important problem of determining the asymptotic behavior as $T \rightarrow \infty$ and then taking the limit $N \rightarrow \infty$. (In comparison, the above analysis characterizes the behavior as $N \rightarrow \infty$ for finite time T.) In general it is not true that the limits $N \rightarrow \infty$ and $T \rightarrow \infty$ can be interchanged. Weak convergence proofs for the asymptotic behavior in $T$ require construction of perturbed Lyapunov functions [167]. In comparison, as explained in [27], since the exponential bound $\sum_{N} \exp (-C N)$ is summable, the BorelCantelli lemma applies. This facilities concluding important results on the exit times of the process $\widetilde{\rho}_{t}^{N}$. In particular, given an open set $U \in \mathbb{R}^{\bar{D}}$, define the exit time as the random variable

$$
\tau^{N}(U)=\inf \left\{t \geq 0: \widetilde{\rho}_{t}^{N} \notin U\right\} .
$$

Let $\gamma^{+}(\rho)$ denote the closure of the set of states visited by the mean field trajectory $\rho_{t}, t \geq 0$. Then, from Theorem 4.5.1, it can be shown that [27, Proposition 1]

$$
\mathbb{P}\left\{\lim _{N \rightarrow \infty} \tau^{N}(U)=\infty\right\}=1
$$

In words, the exit time of the infected process from any neighbourhood of the mean field dynamics trajectory is probabilistically very large. Indeed, Benaïm \& Weibull in [27] go on to conclude that attractors of the mean field dynamics are good predictors of the stochastic process when the initial conditions are close.

\subsubsection{Sentiment-Based Observations}

Next, consider the sensing mechanism with observations defined in $\$ 4.3$. Assuming the sample size is sufficiently large, it follows from the central limit theorem 
(for i.i.d. processes, in the case of independent sampling, and for Markov processes, in the case of MCMC sampling) that

$$
z_{k}(d, y) \approx H(y) \rho_{k}(d)+D(y)+v_{k}(d, y)
$$

where

$$
\begin{aligned}
D(y) & =B_{0 y} P(d), \\
H(y) & =\left(B_{1 y}-B_{0 y}\right) P(d), \\
v_{k}(d, y) & \sim \mathbf{N}\left(0, \sigma^{2}\left(\rho_{k}(d), y\right)\right) .
\end{aligned}
$$

For i.i.d. sampling, the variance is easily evaluated as

$$
\begin{aligned}
\sigma^{2}\left(\rho_{k}(d), y\right)=\frac{1}{\alpha(d)} & \left(H(y) \rho_{k}(d)+D(y)\right) \\
& \times\left(1-H(y) \rho_{k}(d)-D(y)\right) .
\end{aligned}
$$

For MCMC sampling, the variance is evaluated by approximating an infinite series (alternatively, excellent bounds exist [40]).

We can then define the following observation process

$$
z_{k}(y) \stackrel{\text { defn }}{=} \frac{\sum_{d=1}^{\bar{D}} d P(d) z_{k}(d, y)}{\sum_{d=1}^{\bar{D}} d P(d)} .
$$

The key point is that $z_{k}(y)$ is a linear combination of $z_{k}(d, y)$. It then follows from (4.9) that

$$
z_{k}(y)=\theta_{k}+v_{k}(y)
$$

where

$$
\begin{aligned}
v_{k}(y) & \sim \mathbf{N}\left(0, \sigma^{2}(y)\right) \\
\sigma^{2}(y) & =\frac{\sum_{d=1}^{\bar{D}} d P(d) \sigma^{2}\left(\rho_{k}(d), y\right)}{\sum_{d=1}^{\bar{D}} d P(d)} .
\end{aligned}
$$

\subsubsection{Bayesian Filtering Problem}

Given the extrinsic and sentiment observations described above, how can the infected degree distribution $\rho_{k}$ and target state $s_{k}$ be estimated at each time instant? Below we discuss estimation of $\rho_{k}$ and $s_{k}$ as a Bayesian filtering problem. 
The partially observed state space model with continuous time dynamics (4.20) and discrete time observations (4.31) and (4.19), constitutes a system with continuous time dynamics and discrete time observations. Alternatively, the partially observed state space model with discrete time dynamics (4.23) and observation equation (4.31), and (4.19) constitutes a system with discrete time dynamics and discrete time observations. In either case, computing the conditional mean estimate of $s_{t}, \rho_{t}$ in the continuous time case or $s_{k}, \rho_{k}$ in the discrete time case, given the observation sequence $\left(z_{1: k}, o_{1: k}\right)$ is a Bayesian filtering problem. In fact, filtering of such jump Markov linear systems have been studied extensively in the signal processing literature [68, 181] and can be solved via the use of sequential Markov chain Monte-Carlo methods.

For large $N$ in the mean field dynamics (4.23), it is reasonable to expect that a linearized approximation in $\bar{\rho}$ to the dynamics is accurate. Denote the resulting linearized model as

$$
\bar{\rho}_{k+1}=\bar{F}\left(s_{k}\right) \bar{\rho}_{k} .
$$

In the special case when only extrinsic observations $o_{k}$ of $s_{k}$ are available (recall the extrinsic observations are defined in (4.19)), computing the filtered estimate $\mathbf{E}\left\{\theta_{k} \mid o_{1: k}\right\}$ can be done via a finite-dimensional filtering algorithm. Indeed, this model coincides with the so-called image-based tracking model that has widely been studied in signal processing [73, 158, 159, 239].

\subsection{Mean Field Dynamics: Fast Information Diffu- sion and Slow Target State Dynamics}

The assumption here is that the diffusion of information flow, namely $\rho$, in the social network occurs on a faster time-scale compared to the evolution of the target state $s$. That is, the transition matrix $A$ of the Markovian target $s$ is almost identity and the target state displays piecewise constant behavior with infrequent jumps from one state to another. This is made explicit in the following assumption.

Assumption 4.2. The target state $s_{k}$ has transition probability matrix $A=I+$ $\frac{1}{N^{2}} Q$ where $Q$ is a generator matrix.

Considering the diffusion dynamics (4.12) together with Assumption 4.2, it is clear that we have a two-time-scale system, where the target process $s$ evolves on a slower time scale as compared with the information diffusion in the network. 
For this two-time-scale case, we have the following mean field dynamics for the infected distribution:

Suppose Assumption 4.2 holds. Then, for $d=1,2, \ldots, \bar{D}$,

$$
\frac{d \rho_{t}(d)}{d t}=\bar{p}_{01}\left(d, \theta\left(\rho_{t}\right), s_{\tau}\right)-\bar{p}_{10}\left(d, \theta\left(\rho_{t}\right), s_{\tau}\right), 0 \leq t \leq \infty
$$

In the above equation, $s_{\tau}$ is a constant on the time-scale $t$; it evolves as a continuous time Markov chain with generator $Q$ on the slow time scale $\tau$.

A similar exponential probability bound to Theorem 4.5.1 can be proved for the above mean field dynamics system comprised of $\bar{D}$ Markov modulated differential equations (4.33). Also, in analogy to (4.23), for large $N$, the discrete time mean field dynamics read:

$$
\bar{\rho}_{k+1}(d)=\bar{\rho}_{k}(d)+\frac{1}{N}\left[\bar{p}_{01}\left(d, \theta\left(\bar{\rho}_{k}\right), s_{k^{\prime}}\right)-\bar{p}_{10}\left(d, \theta\left(\bar{\rho}_{k}\right), s_{k^{\prime}}\right)\right] .
$$

Here, $k$ indexes a fast time scale and $k^{\prime}$ indexes a slow time scale. In particular, the slow process $s_{k^{\prime}}$ is a constant on the fast time scale $k$; it evolves as a discrete time Markov chain with transition matrix $A=I+\frac{1}{N^{2}} Q$ on the slow time scale $k^{\prime}$.

The sentiment based observation process is modelled as in (4.31), and the extrinsic observation process $o_{k^{\prime}}$ on the slow time-scale $k^{\prime}$ is modelled as in (4.19).

\subsubsection{Bayesian Filtering Problem}

In the case of fast information diffusion and slow target dynamics, estimating the slow target state $s$ and fast infected distribution $\rho$ are decoupled due to the two-time-scale nature of the problem. In particular, at the slow time-scale $k^{\prime}$, the conditional mean estimate $\mathbf{E}\left\{s_{k^{\prime}} \mid o_{1: k^{\prime}}\right\}$ is obtained using a Hidden Markov model filter (or Wonham filter in continuous time) [78]. Then, estimating the state $\rho_{k}$ given noisy observations $z_{k}(y)$ becomes a nonlinear regression problem since there is no state noise process driving (4.23). Alternatively, a stochastic approximation algorithm can be used to track the Markov chain; see [259] for details.

\subsubsection{Numerical Example}

Below we illustrate the mean field dynamics model for the case of fast information diffusion and slow target dynamics. In this example, we simulate the diffusion of 
information through a network comprising of $N=100$ nodes (with maximum degree $\bar{D}=17$ ) in two different scenarios as described below. We further assume that at time $k=0,5 \%$ of nodes are infected. The mean field dynamics model is investigated in terms of the infected link probability (4.9). The infected link probability for the case of fast information diffusion and slow target dynamics can be computed by

$$
\theta\left(\bar{\rho}_{k}\right)=\frac{\sum_{d=1}^{\bar{D}} d P(d) \bar{\rho}_{k}(d)}{\sum_{d}^{\bar{D}} d P(d)}
$$

Here, $\bar{\rho}_{k}$ is obtained via discrete-time mean field dynamics (4.34).

Scenario 1: Assume each agent is a myopic optimizer and, hence, chooses to adopt the technology only if $c^{(m)}>A_{k}^{(m)} ; r=1$. At time $k$, the costs $c^{(m)}$, $m=1,2, \ldots, 100$, are i.i.d. random variables simulated from uniform distribution $U\left[0, C\left(s_{k}\right)\right]$. Therefore, the transition probabilities in (4.5) are

$$
p_{01}\left(d, A_{k}^{(m)}, s_{k}\right)=\mathbb{P}\left(c^{(m)}>A_{k}^{(m)}\right)= \begin{cases}\frac{\left.A_{k}^{(m)}\right)}{C\left(s_{k}\right)}, & A_{k}^{(m)} \leq C\left(s_{k}\right), \\ 1, & A_{k}^{(m)}>C\left(s_{k}\right) .\end{cases}
$$

The probability that a product fails is $p_{F}=0.3$, i.e.,

$$
p_{10}\left(d, A_{k}^{(m)}, s_{k}\right)=0.3 .
$$

The infected link probabilities obtained from network simulation (4.9) and from the discrete-time mean field dynamics model (4.35) are illustrated in Figure 4.1a, To illustrate that the infected link probability computed from (4.35) follows the true one (obtained by network simulation), we assume that the value of $C$ jumps from 1 to 10 at time $k=200$, and from 10 to 1 at time $k=500$. As can be seen in Figure 4.1a, the mean field dynamics provide an excellent approximation to the true infected distribution.

Scenario 2: The transition probabilities in (4.5) depend on the node's connectivity $d$. These probabilities are

$$
p_{01}\left(d, A_{k}^{(m)}, s_{k}\right)= \begin{cases}\frac{\left.A_{k}^{(m)}\right)}{d C\left(s_{k}\right)}, & A_{k}^{(m)} \leq d C\left(s_{k}\right) \\ 1, & A_{k}^{(m)}>d C\left(s_{k}\right)\end{cases}
$$

and

$$
p_{10}\left(d, A_{k}^{(m)}, s_{k}\right)=0.3
$$

The infected link probability obtained from network simulation (4.9) and from the discrete-time mean field dynamics model (4.35) are illustrated in Figure 4.1b. 


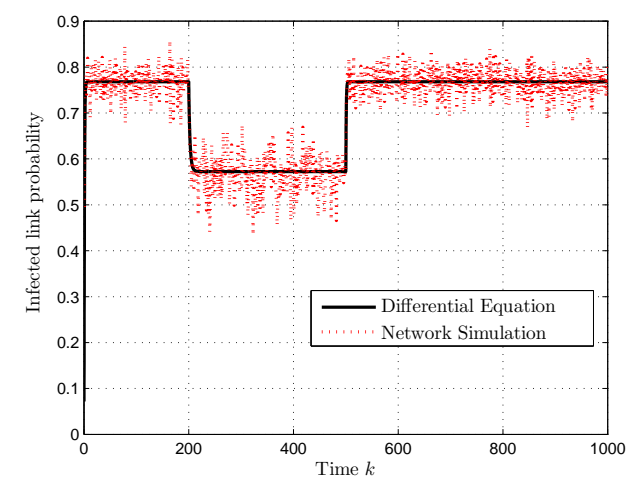

(a)

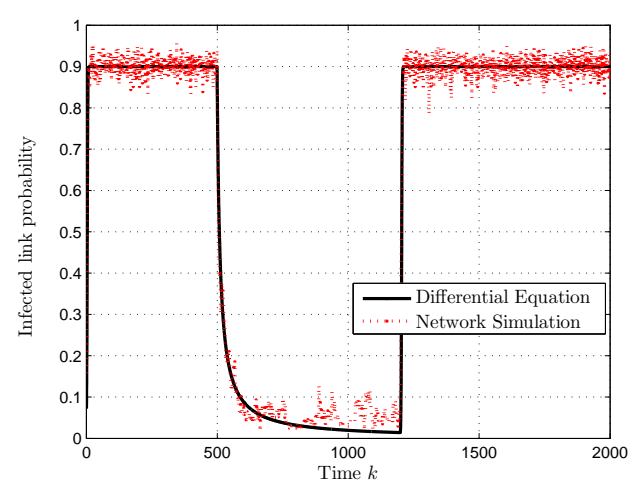

(b)

Figure 4.1: The infected link probability obtained from network simulation compared to the one obtained from the mean field dynamics model (4.35) in two different scenarios: (a) The transition probabilities in (4.13) depend only on the number of infected neighbors $\left.A^{(} m\right)_{k}$ (the parameters are defined in Scenario 1). (b) The transition probabilities in (4.13) depend on the node's connectivity $d$ and the number of infected neighbors $A_{k}^{(m)}$ (the parameters are defined in Scenario 2).

Similar to Scenario 1, here, we assume that the value of $C$ jumps from 1 to 10 at time $k=500$, and from 10 to 1 at time $k=1200$ to show that the infected link probability computed from (4.35) follows the one obtained from network simulation. Again the mean field dynamics provide an excellent approximation to the actual infected distribution.

\subsection{Closing Remarks}

This chapter has shown how the dynamics of the spread of information in a graph can be asymptotically modelled via Markov modulated differential equations. These mean field dynamics serve as a tractable model for filtering of underlying states of the network. As an immediate extension, one can consider sequential change detection and, more generally, control of the mean field dynamics.

Below we outline two extensions. 


\section{Reactive Information Diffusion}

A key difference between social sensors and conventional sensors in statistical signal processing is that social sensors are reactive: A social sensor uses additional information gained to modify its behavior. Consider the case where the sentiment-based observation process is made available in a public blog. Then, these observations will affect the transition dynamics of the agents and, therefore, the mean field dynamics.

\section{How Does Connectivity Affect Mean Field Equilibrium?}

The papers [183, 184] examine the structure of fixed points of the mean field differential equation (4.20) when the underlying target process $s$ is not present (equivalently, $s$ is a one state process). They consider the case where the agent transition probabilities are parameterized by $p_{01}(d, a)=\mu F(d, a)$ and $p_{10}=p_{F}$; see (4.14). Then, defining $\lambda=\mu / p_{F}$, they study how the following two thresholds behave with the degree distribution and diffusion mechanism:

1. Critical threshold $\lambda_{c}$ : This is defined as the minimum value of $\lambda$ for which there exists a fixed point of (4.20) with positive fraction of infected agents, i.e., $\rho_{\infty}(d)>0$ for some $d$ and, for $\lambda \leq \lambda_{c}$, such a fixed point does not exist.

2. Diffusion threshold $\lambda_{d}$ : Suppose the initial condition $\rho_{0}$ for the infected distribution is infinitesimally small. Then, $\lambda_{d}$ is the minimum value of $\lambda$ for which $\rho_{\infty}(d)>0$ for some $d$, and such that, for $\lambda \leq \lambda_{d}, \rho_{\infty}(d)=0$ for all $d$.

Determining how these thresholds vary with degree distribution and diffusion mechanism is very useful for understanding the long term behavior of agents in the social network. 


\section{Chapter 5}

\section{Non-Cooperative Game-Theoretic Learning}

\subsection{Introduction}

Many social and economic situations involve interactive decision making with possibly diverging interests. Often there is an advantage to having individuals coordinate on their decisions [264]. For instance, a person may choose the same cellphone carrier as the majority of family and friends to take advantage of the free talk times. Social networks spread information, thereby facilitate coordination of such self-interested units by speeding up the resolution of uncertainties. For instance, individuals form friendship groups within which members are able to observe others' decisions.

This chapter examines how global coordination can be obtained among a group of decision makers when each decision maker has limited awareness of the outside world and is only capable of 'simple' local behavior. Such coordination can potentially lead to higher social welfare to each individual decision maker, and hence equilibrates the social network. We focus on no (or low) internal regret algorithms as a strategy of play in game-theoretic learning. The internal regret 1 compares the loss of a strategy to the loss of a modified strategy, which consistently replaces one action by another-for example, "every time you bought Windows, you should have bought Apple instead." We refer the reader to [32] for an excellent discussion of internal and external regret-based algorithms.

\footnotetext{
${ }^{1}$ In comparison, the external regret compares the performance of a strategy selecting actions to the performance of the best of those actions in hindsight.
} 
Game theory has traditionally been used in economics and social sciences with a focus on fully rational interactions where strong assumptions are made on the information patterns available to individual agents. In comparison, social sensors are agents with partial information and it is the dynamic interactions between agents that are of interest. This, together with the interdependence of decisionmakers' choices, motivates the need for game-theoretic learning models for agents interacting in social networks.

Learning dynamics in games can be typically classified into Bayesian learning [1, 52], adaptive learning [118], and evolutionary dynamics [127, 178]. This chapter focuses on adaptive learning where individuals deploy simple rule-ofthumb strategies. The aim is to determine if such simple individual behaviour can result in sophisticated global behaviour. We finish this chapter with an example to show how the presented regret-based rule-of-thumb strategies can be employed to devise an energy-aware sensing mechanism for parameter estimation va diffusion least mean squares.

\subsubsection{Literature}

The theory of learning in games formalizes the idea that equilibrium arises as a result of players learning from experience. It further examines how and what kind of equilibrium might arise as a consequence of a long-run process of adaptation and learning in an interactive environment [94]. Due to space limitations, this chapter focuses on adaptive learning models in which players try to maximize their own payoffs while simultaneously learning about others' play? The question is then when self-interested learning and adaptation will result in the emergence of equilibrium behavior. The literature on game-theoretic learning has relied on the idea that learning rules should strike a balance between performance and complexity [94]. That is, simple learning rules are expected to perform well in simple environments, whereas larger and more complex environments call for more sophisticated learning mechanisms.

The simplest setting is one in which players' decisions can be observed by all other players at the end of each round. Fictitious play [92] is a simple stylized model of learning in non-cooperative repeated games with such communication model. The player behaves as if she is Bayesian. That is, she believes that the

\footnotetext{
${ }^{2}$ The evolutionary game theory also provides a rich framework for modeling the dynamics of adaptive opponent strategies for large population of players. The interested reader is referred to [127, 223] for recent surveys.
} 
opponents' play corresponds to draws from an unknown stationary distribution and simply best responds to her belief about such a distribution 3 . Another simple model, namely, stochastic fictitious play [92], forms beliefs as in fictitious play, however, chooses actions according to a stochastic best-response function. This new model brings about two advantages: (i) it avoids the discontinuity inherent in fictitious play, where a small change in the belief can lead to an abrupt change in behavior; (ii) it is "Hannan-consistent" [115]: its time average payoff is at least as good as maximizing against the time-average of opponents' play, which is not true for fictitious play.

Regret-matching [117, 119] as a strategy of play in long-run interactions has been long known to guarantee convergence to the correlated equilibria set [20]. The regret-based adaptive procedure in [117] assumes a complete connectivity graph 4 for information exchange among players, whereas the regret-based reinforcement learning algorithm in [119] assumes a set of isolated players who rely only on their realized payoffs. This chapter is inspired by the work of Hart \& Mas-Colell in [117, 119] and focuses on learning algorithms in scenarios where players form social groups and disclose information of their decisions only within their social groups.

The concept of regret, well-known in the decision theory, has also been introduced to the realm of random sampling [70, 71]. These methods are of particular interest in the multi-armed bandit literature, which is concerned with optimizing the cumulative objective function values realized over a period of time [16, 17], and the pure exploration problem [15], which involves finding the best arm after a given number of arm pulls. In such problems, the regret value of a candidate arm measures the worst-case consequence that might possibly result from selecting another arm. Such a regret is sought to be minimized via devising random sampling schemes that establish a proper balance between exploration and exploitation.

\subsubsection{Experimental Studies}

Parallel to the growing interest in social networks, a new game-theoretic paradigm has been developed to incorporate some aspects of social networks such as information exchange and influence structure into a game formalism. One such example is the so-called graphical games, where each player's influence is restricted to

\footnotetext{
${ }^{3}$ Obviously, if all players make decisions according to the fictitious play, the actual environment is not stationary, hence, players have the wrong model of the environment.

${ }^{4} \mathrm{~A}$ complete graph is a simple undirected graph in which every pair of distinct vertices is connected by a unique edge.
} 
his immediate neighbors [139, 140]. Early experimental studies on these games indicate significant network effects. For instance, Kearns et al. present in [141] the results of experiments in which human subjects solved a version of the vertex coloring problem 5 that was converted into a graphical game. Each participant had control of a single vertex in the network. One task was to coordinate (indirectly) with their neighbors so that they all had the same color.

Griffin \& Squicciarini in [109] tackle the problem of information release in social media via a game-theoretic formulation. They analyze the dynamics of social identity verification protocols and, based on some real-world data, develop a deception model for online users. The proposed model captures a user's willingness to release, withhold or lie about information depending on the behavior of the user's circle of friends. The empirical study infers a relationship between the qualitative structure of the game equilibria and the automorphism group of the social network.

The correlated equilibrium arguably provides a natural way to capture conformity to social norms [46]. It can be interpreted as a mediator instructing people to take actions according to some commonly known probability distribution. Such a mediator can be thought of as a social norm that assigns roles to individuals in a society [257]. If it is in the interests of each individual to assume the role assigned to him by the norm, then the probability distribution over roles is a correlated equilibrium [20, 67, 89]. The Fact that actions are conditioned on signals or roles indicates how conformity can lead to coordinated actions within social groups [134, 228]. Norms of behavior are important in various real-life behavioral situations such as public good provision, resource allocation, and the assignment of property rights; see [38] for an extensive treatment of public good games in social networks.

The works [47, 72] further report results from an experiment that explores the empirical validity of correlated equilibrium. It is found in [72] that when the private recommendations from the mediator are not available to the players, the global behavior is characterized well by mixed-strategy Nash equilibrium. Their main finding, however, was that players follow recommendations from the third

\footnotetext{
${ }^{5}$ The vertex coloring problem is an assignment of labels (colors) to the vertices of a graph such that no two adjacent vertices share the same label. The model has applications in scheduling, register allocation, pattern matching, and community identification in social networks [241, 189].

${ }^{6}$ The public good game is a standard of experimental economics. In the basic game, players secretly choose how many of their private tokens to put into a public pot and keep the rest. The tokens in this pot are multiplied by a factor (greater than one) and this "public good" payoff is evenly divided among players.
} 
party only if those recommendations are drawn from a correlated equilibrium that is "payoff-enhancing" relative to the available Nash equilibria.

\subsection{Non-Cooperative Games and Correlated Equilibrium}

This section starts with introducing the standard representation of non-cooperative games in $\$ 5.2 .1$. We then proceed to introduce and elaborate on two prominent solution concepts of such games in $\$ 5.2 .2$.

\subsubsection{Strategic Form Non-Cooperative Games}

The initial step in performing a non-cooperative game-theoretic analysis is to frame the situation in terms of all conceivable actions of agents and their associated payoffs. The standard representation of a non-cooperative game $\mathcal{G}$, known as normal form or strategic form game [194, 206], comprises three elements:

$$
\mathcal{G}=\left(\mathcal{N},\left(\mathcal{A}^{n}\right)_{n \in \mathcal{N}},\left(U^{n}\right)_{n \in \mathcal{N}}\right)
$$

Each element is described as follows:

(i) Player Set: $\mathcal{N}=\{1, \cdots, N\}$. Essentially, a player models an entity that is entitled to making decisions, and whose decisions affect others' decisions. Players may be people, organizations, firms, etc., and are indexed by $n \in \mathcal{N}$.

(ii) Action Set: $\mathcal{A}^{n}=\left\{1, \cdots, A^{n}\right\}$, that denotes the actions, also referred to as pure strategies, available to player $n$ at each decision point. A generic action taken by player $n$ is denoted by $\mathrm{a}^{n}$, where $\mathrm{a}^{n} \in \mathcal{A}^{n}$. The actions of players may range from deciding to establish or abolish links with other agents [22] to choosing among different products/technologies [269].

Subsequently, a generic element of the action profile of all players is denoted by $\mathbf{a}=\left(\mathrm{a}^{1}, \cdots, \mathrm{a}^{N}\right)$, and belongs to the set $\mathcal{A}=\mathcal{A}^{1} \times \cdots \times \mathcal{A}^{N}$, where $\times$ denotes the Cartesian product. Following the common notation in game theory, one can rearrange the action profile as $\mathbf{a}=\left(\mathrm{a}^{n}, \mathbf{a}^{-n}\right)$, where $\mathbf{a}^{-n}=\left(\mathrm{a}^{1}, \cdots, \mathrm{a}^{n-1}, \mathrm{a}^{n+1}\right.$, $\left.\cdots, \mathrm{a}^{N}\right)$ denotes the action profile of all players excluding player $n$.

(iii) Payoff Function: $U^{n}: \mathcal{A} \rightarrow \mathbb{R}$ is bounded, and gives payoff to player $n$ as a function of the action profile $\mathbf{a} \in \mathcal{A}$ taken by all players. The interpretation of such a payoff is the sum of rewards and costs associated with the chosen action as the outcome of the interaction. The payoff function can be quite general. For 
instance, it could reflect reputation or privacy, using the models in [110, 197], or benefits and costs associated with maintaining links in a social network, using the model in [22, 266]. It could also reflect benefits of consumption and the costs of production, download, and upload in content production and sharing over peerto-peer networks [107, 210], or the capacity available to users in communication networks [135, 173].

In contrast to the pure strategy that specifies a possible decision or action, a mixed strategy is a probability distribution that assigns to each available action in the action set a likelihood of being selected. More precisely, a mixed strategy for player $n$ is of the form

$$
\mathbf{p}^{n}=\left(p^{n}(1), \cdots, p^{n}\left(A^{n}\right)\right), \quad 0 \leq p^{n}(i) \leq 1, \sum_{i \in \mathcal{A}^{n}} p^{n}(i)=1 .
$$

A mixed strategy can be interpreted as how frequently each action is to be played in a repeated game. Note that, even if the set of pure strategies is finite, there are infinitely many mixed strategies. The set of all mixed strategies available to player $n$ forms an $A^{n}$-simplex, denoted by $\Delta \mathcal{A}^{n}$. An individual uses a mixed strategy only when: (i) he/she is indifferent between several pure strategies; (ii) keeping the opponent guessing is desirable, i.e., the opponent can benefit from knowing the next move; (iii) the player needs to experiment in order to learn his/her optimal strategy. The significance of mixed strategies becomes more clear when we talk about existence of Nash equilibrium in $\$ 5.2 .2$.

In framing the game-theoretic analysis, another important issue is the information available to individuals at each decision point. This essentially has bearing on the timing of interactions and is ascertained via answering the following questions:

(i) Is the interaction repeated? Repeated games refer to a situation where the same base game, referred to as stage game, is repeatedly played at successive decision points. For instance, suppliers and buyers make deals repeatedly, nations engage in ongoing trades, bosses try to motivate workers on an ongoing basis, etc. Repeated interaction allows for socially beneficial outcomes, essentially substituting for the ability to make binding agreements. It further allows learning as the outcome of each interaction conveys information about actions and preferences of other players.

(ii) Do players make decisions simultaneously or sequentially? Simultaneous move games arise when players make decisions simultaneously, without being aware of the choices and preferences of others. For instance, two firms independently decide whether to develop and market a new product, or individuals 
independently make a choice as which social club to join. In contrast, moving after another player in sequential games gives the advantage of knowing and accounting for the previous players' actions prior to taking an action.

(iii) What does each player realizelobserve once the interaction is concluded? Embodied in the answer to this question is the social knowledge of each player. With the prevalence of social networks, individuals may obtain information about the preferences and choices of (some of) other decision makers in addition to the payoff that they realize as the outcome of making choices. A particular choice made by a self-interested rational player is understood to be a best response to his limited perception of the outside world. Observing such choices, other players could adjust their strategies so as to maximize their payoffs via decreasing others.

In this chapter, we concentrate on simultaneous move non-cooperative games that are infinitely repeated. Three different models will be studied for the information flow and social knowledge of players. The first model assumes social agents who observe the decisions of all agents playing the same game. The second model considers social groups, within which agents share their decisions. Hence, each agent is only aware of successive decisions of a subset of the agents who play the same game. The third model assumes formation of homogeneous social groups, where agents share identical interests and exchange their beliefs.

\subsubsection{Solution Concepts: Correlated vs. Nash Equilibrium}

Having framed the interactions as a non-cooperative game, one can then make predictions about the behavior of decision makers. Strategic dominance is the most powerful analytical tool to make behavioral predictions. A dominant strategy is one that ensures the highest payoff for an agent irrespective of the action profile of other players. A more precise definition is provided below.

Definition 5.1 (Dominant Strategy). An action $a_{d}^{n} \in \mathcal{A}^{n}$ is a dominant strategy for player $n$ if for all other actions $a^{n} \in \mathcal{A}^{n}-\left\{a_{d}^{n}\right\}$ :

$$
U^{n}\left(a_{d}^{n}, \mathbf{a}^{-n}\right)>U^{n}\left(a^{n}, \mathbf{a}^{-n}\right), \quad \forall \mathbf{a}^{-n} \in \mathcal{A}^{-n}
$$

Dominant strategies make game-theoretic analysis relatively easy. They are also powerful from the decision makers perspective since no prediction of others' behavior is required. However, such strategies do not always exist, hence, one needs to resort to equilibrium notions in order to make behavioral predictions. 
The Nash equilibrium [199] and its refinements are without doubt the most well-known game-theoretic equilibrium notion in both economics and engineering. One can classify Nash equilibria into two types: (i) Pure strategy Nash equilibria, where all agents are playing pure strategies, and (ii) Mixed strategy Nash equilibria, where at least one agent is playing a mixed strategy. The underlying assumption in Nash equilibrium is that players act independently. That is, the probability distribution on the action profiles is a product measure. More precisely,

$$
p\left(i_{1}, \cdots, i_{N}\right) \stackrel{\text { defn }}{=} \mathbb{P}\left(\mathrm{a}^{1}=i_{1}, \cdots, \mathrm{a}^{N}=i_{N}\right)=\prod_{k=1}^{N} p^{n}\left(i_{n}\right)
$$

where $p^{n}\left(i_{n}\right)$ denotes the probability of agent $n$ playing action $i_{n}$.

A Nash equilibrium is a strategy profile with the property that no single player can benefit by deviating unilaterally to another strategy; see below for precise definitions.

Definition 5.2 (Nash Equilibrium). An action profile $\mathbf{a}=\left(a^{n}, \mathbf{a}^{-n}\right)$ is a pure strategy Nash equilibrium if

$$
U^{n}\left(a^{n}, \mathbf{a}^{-n}\right) \geq U^{n}\left(\underline{a}^{n}, \mathbf{a}^{-n}\right), \quad \forall \underline{a}^{n} \in \mathcal{A}^{n}, \forall n \in \mathcal{N} .
$$

A profile of mixed strategies $\mathbf{p}=\left(\mathbf{p}^{1}, \cdots, \mathbf{p}^{N}\right)$ forms a mixed strategy Nash equilibrium if for all $\underline{a}^{n} \in \mathcal{A}^{n}$ and $n \in \mathcal{N}$ :

$$
\sum_{a^{n}, \mathbf{a}^{-n}} p\left(a^{n}, \mathbf{a}^{-n}\right) U^{n}\left(a^{n}, \mathbf{a}^{-n}\right) \geq \sum_{\mathbf{a}^{-n}} p\left(\underline{a}^{k}, \mathbf{a}^{-n}\right) U^{n}\left(\underline{a}^{n}, \mathbf{a}^{-n}\right) .
$$

John F. Nash proved in his famous paper [199] that every game with a finite set of players and actions has at least one mixed strategy Nash equilibrium. However, as asserted by Robert J. Aumann7 in the following extract from [20], "Nash equilibrium does make sense if one starts by assuming that, for some specified reason, each player knows which strategies the other players are using." Evidently, this assumption is rather restrictive and, more importantly, is rarely true in any strategic interactive situation. He adds:

"Far from being inconsistent with the Bayesian view of the world, the notion of equilibrium is an unavoidable consequence of that view.

\footnotetext{
${ }^{7}$ Robert J. Aumann was awarded the Nobel Memorial Prize in Economics in 2005 for his work on conflict and cooperation through game-theoretic analysis. He is the first to conduct a full-fledged formal analysis of the so-called infinitely repeated games.
} 
It turns out, though, that the appropriate equilibrium notion is not the ordinary mixed strategy equilibrium of Nash (1951), but the more general notion of correlated equilibrium.” - Robert J. Aumann

This, indeed, is the very reason why correlated equilibrium [20] best suits and is central to the analysis of strategic decision-making in social networks in this chapter.

Correlated equilibrium is a generalization of the well-known Nash equilibrium and describes a condition of competitive optimality among decision makers. It can most naturally be viewed as imposing equilibrium conditions on the joint action profile of agents (rather than on individual actions as in Nash equilibrium). An intuitive interpretation of correlated equilibrium is coordination in decisionmaking as described by the following example: Consider a traffic game where two drivers meet at an intersection and have to decide whether to "go" or "wait". If they simultaneously decide to go, they will crash and both incur large losses. If one waits and the other one goes, the one that passes the intersection receives positive utility, whereas the one who waits receive zero utility. Finally, if they both decide to wait, they both will receive small negative utilities due to wasting their time. The natural solution is the traffic light. In game-theoretic terminology, the traffic light serves as a fair randomizing device that recommends actions to the agents-signals one of the cars to go and the other one to wait; it is fair in the sense that at different times it makes different recommendations.

In light of the above example, suppose that a mediator is observing a noncooperative game being repeatedly played among a number of agents. The mediator, at each round of the game, gives private recommendations as what action to choose to each agent. The recommendations are correlated as the mediator draws them from a joint probability distribution on the action profile of all agents; however, each agent is only given recommendations about her own action. Each agent can freely interpret the recommendations and decide if to follow. A correlated equilibrium results if neither of agents wants to deviate from the provided recommendation. That is, in correlated equilibrium, agents' decisions are coordinated as if there exists a global coordinating device that all agents trust to follow.

Let us now formally define the set of correlated equilibrium for the game $\mathcal{G}$, defined in $\$ 5.2 .1$.

Definition 5.3 (Correlated Equilibrium). Let $\mathrm{p}$ denote a probability distribution on the space of action profiles $\mathcal{A}$, i.e.,

$$
0 \leq p(\mathbf{a}) \leq 1, \forall \mathbf{a} \in \mathcal{A} \text {, and } \sum_{\mathbf{a}} p(\mathbf{a})=1 \text {. }
$$


The set of correlated $\epsilon$-equilibria is the convex polytope

$$
\mathcal{C}_{\epsilon}=\left\{\mathbf{p}: \sum_{\mathbf{a}^{-n}} p^{n}\left(i, \mathbf{a}^{-n}\right)\left[U^{n}\left(j, \mathbf{a}^{-n}\right)-U^{n}\left(i, \mathbf{a}^{-n}\right)\right] \leq \epsilon, \forall i, j \in \mathcal{A}^{n}, n \in \mathcal{N}\right\}
$$

where $p^{n}\left(i, \mathbf{a}^{-n}\right)$ denotes the probability of player $n$ playing action $i$ and the rest playing $\mathbf{a}^{-n}$. If $\epsilon=0$ in (5.8), the convex polytope represents the set of correlated equilibria and is denoted by $\mathcal{C}$.

There is much to be said about correlated equilibrium; see Aumann [18, 20] for rationality arguments. Some advantages that make it ever more appealing include:

1. Realistic: Correlated equilibrium is realistic in multi-agent learning. Indeed, Hart and Mas-Colell observe in [119] that for most simple adaptive procedures, "... there is a natural coordination device: the common history, observed by all players. It is thus reasonable to expect that, at the end, independence among players will not obtain;"

2. Structural Simplicity: The correlated equilibria set constitutes a compact convex polyhedron, whereas the Nash equilibria are isolated points at the extrema of this set [200];

3. Computational Simplicity: Computing correlated equilibrium only requires solving a linear feasibility problem (linear program with null objective function) that can be done in polynomial time, whereas computing Nash equilibrium requires finding fixed points;

4. Payoff Gains: The coordination among agents in the correlated equilibrium can lead to potentially higher payoffs than if agents take their actions independently (as required by Nash equilibrium) [20];

5. Learning: There is no natural process that is known to converge to a Nash equilibrium in a general non-cooperative game that is not essentially equivalent to exhaustive search. There are, however, natural processes that do converge to correlated equilibria (the so-called law of conservation of coordination [120]), e.g., regret-matching [117].

Existence of a centralized coordinating device neglects the distributed essence of social networks. Limited information at each agent about the strategies of others further complicates the process of computing correlated equilibria. In fact, 


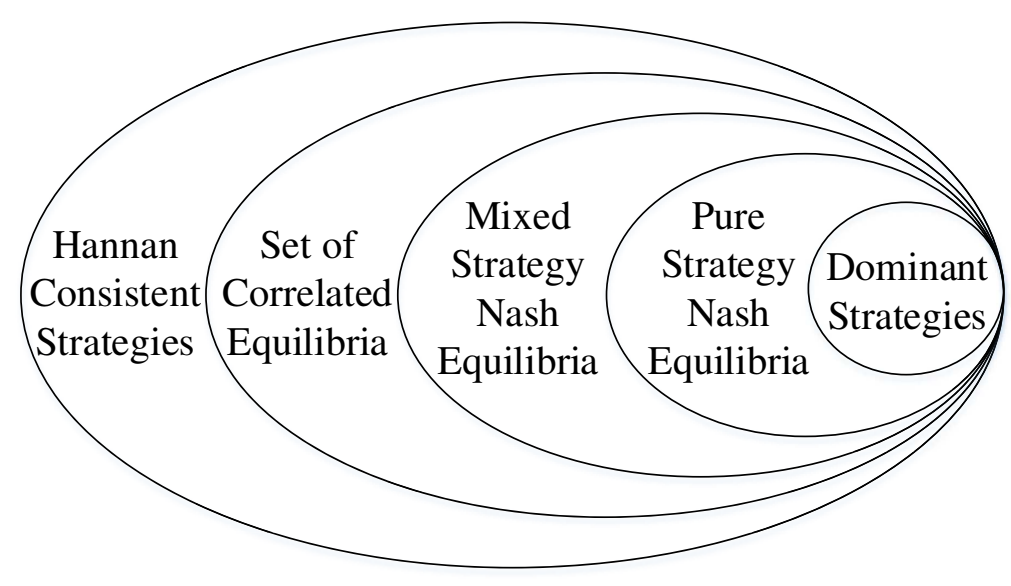

Figure 5.1: Equilibrium notions in non-cooperative games. Enlarging the equilibria set weakens the behaviorial sophistication on the player's part to distributively reach equilibrium through repeated plays of the game.

even if agents could compute correlated equilibria, they would need a mechanism that facilitates coordinating on the same equilibrium state in the presence of multiple equilibria - each describing, for instance, a stable coordinated behavior of manufacturers on targeting influential nodes in the competitive diffusion process [248]. This highlights the significance of adaptive learning algorithms that, through repeated interactive play and simple strategy adjustments by agents, ensure reaching correlated equilibrium. The most well-known of such algorithms, fictitious play, was first introduced in 1951 [219], and is extensively treated in [92]. It, however, requires monitoring the behavior of all other agents that contradicts the information exchange structure in social networks. The focus of this chapter is on the more recent regret-matching learning algorithms [26, 42, 117, 119]. We use tools from stochastic approximation [167], to adapt these algorithms to the information exchange structure in social networks, and Markov-switched systems [261, 263], to track time-varying equilibria under evolution of the environment and the social network.

Figure 5.1 illustrates how the various notions of equilibrium are related in terms of the relative size and inclusion in other equilibria sets. As discussed earlier in this subsection, dominant strategies and pure strategy Nash equilibria do not always exist - the game of "Matching Pennies" being a simple example. Every 
finite game, however, has at least one mixed strategy Nash equilibrium. Therefore, the "nonexistence critique" does not apply to any notion that generalizes the mixed strategy Nash equilibrium in Figure 5.1. A Hannan consistent strategy (also known as "universally consistent" strategies [91]) is one that ensures, no matter what other players do, the player's average payoff is asymptotically no worse than if she were to play any constant strategy for in all previous periods. Hannan consistent strategies guarantee no asymptotic external regrets and lead to the so-called "coarse correlated equilibrium" [195] notion that generalizes the Aumann's correlated equilibrium.

\subsection{Local Adaptation and Learning}

This section presents an adaptive decision-making procedure that, if locally followed by every individual decision maker, leads to rational global behavior. The global behavior is meant to capture the decision strategies of all individuals taking part in the decision-making process, and will be made clear in the next section. This procedure is viewed as particularly simple and intuitive as no sophisticated updating, prediction, or fully rational behavior is essential on the part of agents. The procedure can be simply described as follows: At each period, an agent may either continue with the same action as the previous period, or switch to other actions, with probabilities that are proportional to a "regret measure" [117]; see Figure 5.2. Willingness to proceed with the previous decision mimics the "inertia" that is existent in human's decision-making process. In what follows, we present three algorithms, in the ascending level of sophistication, that adapt this simple procedure to various social network architectures.

The communication among agents (hence, the level of "social knowledge") can be captured by a connectivity graph . Below, a formal definition is given:

Definition 5.4 (Connectivity Graph). It is an undirected graph $G=(V, E)$, where $V=\mathcal{N}$ is the set of agents, and

$$
n, l \in E \Leftrightarrow n \text { knows } a_{k}^{l} \text { and } l \text { knows } a_{k}^{n} \text { at the end of period } k \text {. }
$$

Accordingly, the neighborhood of each agent $n$ is defined as:

$$
\begin{array}{ll}
\text { Open Neighborhood: } & N^{n} \stackrel{\text { defn }}{=}\{l \in \mathcal{N} ;(n, l) \in E\} \\
\text { Closed Neighborhood: } & N_{c}^{n} \stackrel{\text { defn }}{=} N^{n} \cup\{n\}
\end{array}
$$




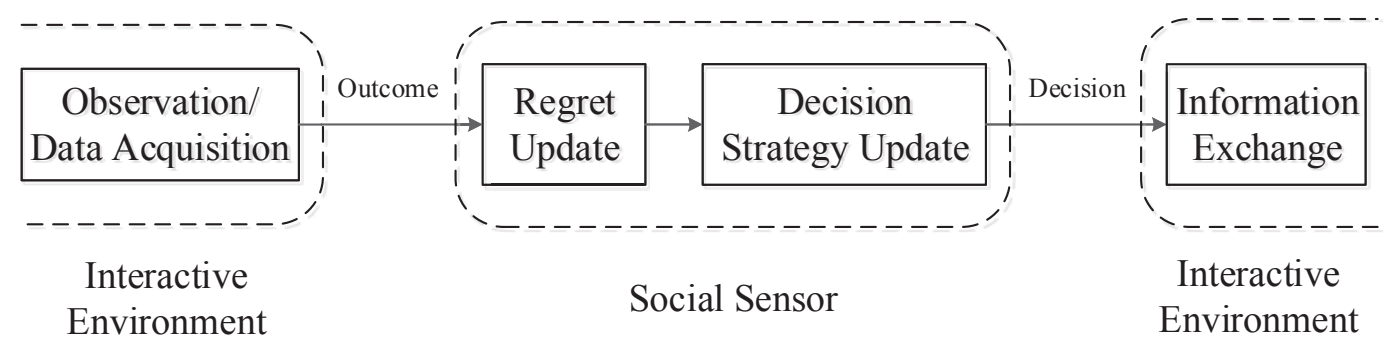

Figure 5.2: Regret-based local adaptation and learning. Individuals update regrets after observing the outcome of the previous decisions and, accordingly, make next decisions. These new decisions will then be shared within social groups.

The communication protocol which allows agents to exchange information only with neighbors is referred to as neighborhood monitoring [102]. This generalizes the perfect monitoring assumption, standard in the game theory literature [118], where agents share decisions with all other agents at the end of each period 8 .

\subsubsection{Case I: Fully Social Players}

For simplicity of exposition, we start with the adaptive learning procedure for the scenario with the highest level of social knowledge: perfect monitoring. That is, once a decision is made by an agent, it is observable by all other agents? 9 . More precisely,

$$
\text { The graph } G \text { is complete and } N_{c}^{n}=\mathcal{N} \text {. }
$$

Although this is a rather impractical assumption in the emerging large-scale social networks, it sheds light and provides insight on the grounds of the decisionmaking procedure and how it can be deployed in environments with more complex connectivity architecture.

\footnotetext{
${ }^{8}$ Note that sharing actions differs from exchanging strategies according to which decisions are made; the latter is more cumbersome and less realistic, however, conveys information about the payoff functions of opponents, hence, can lead to faster coordination.

${ }^{9}$ In certain situations, the aggregate decisions of all agents affects individual agent's strategy update, e.g., proportion of neighbors adopting a new technology or proportion of neighboring sensors that have chosen to activate. Therefore, one can assume that this aggregate information is available to each agent (instead of individual decisions) at the end of each period.
} 
Suppose the game $\mathcal{G}$, defined in $\$ 5.2 .1$, is being repeatedly played over time: $k=1,2, \ldots$. At time $k$, each agent $n$ makes a decision $\mathrm{a}_{k}^{n} \in \mathcal{A}^{n}$ according to a probability distribution $\mathbf{p}_{k}^{n}$, which is defined via the following "regret-matching" procedure [117]:

Let $I_{\{\cdot\}}$ denote the indicator operator and $0<\varepsilon<1$ be a small parameter. Define the matrix $R_{k}^{n}$, where each element $r_{k}^{n}(i, j)$ records

$$
r_{k}^{n}(i, j)=\varepsilon \sum_{\tau \leq k}(1-\varepsilon)^{k-\tau}\left[U^{n}\left(j, \mathbf{a}_{\tau}^{-n}\right)-U^{n}\left(\mathbf{a}_{\tau}\right)\right] I\left\{\mathbf{a}_{\tau}^{n}=i\right\}
$$

The above expression for $r_{k}^{n}(i, j)$ has a clear interpretation as a measure of the "regret" that agent $n$ would experience for not having played action $j$, every time action $i$ was picked in the past. The strategy $\mathbf{p}_{k}^{n}=\left(p_{k}^{n}(1), \cdots, p_{k}^{n}\left(A^{n}\right)\right)$ is then defined as follows

$$
\begin{aligned}
p_{k}^{n}(i) & \stackrel{\text { defn }}{=} \mathbb{P}\left(\mathrm{a}_{k}^{n}=i \mid \mathrm{a}_{k-1}^{n}=j\right) \\
& = \begin{cases}\frac{1}{\mu^{n}}\left|r_{k}^{n}(j, i)\right|^{+}, & i \neq j \\
1-\sum_{j \neq i} p_{k}^{n}(j), & i=j\end{cases}
\end{aligned}
$$

where $|x|^{+}=\max \{0, x\}$, and $\mu^{n}>0$ is a large enough number so that $\mathbf{p}_{k}^{n}$ is a valid probability mass function 10 .

Intuitively speaking, the regret-matching procedure governs the decision-making process by propensities to depart from the current decision. It is natural to postulate that, if a decision maker decides to switch her action, it should be to actions that are perceived as being better. The regret-matching procedure assigns positive probabilities to all such better choices. In particular, the better an alternative action seems, the higher will be the probability of choosing it next time. More precisely, the probabilities of switching to different actions are proportional to their regrets relative to the current action.

The regret-matching procedure in the case of fully social players is summarized in Algorithm 5 ,

\footnotetext{
${ }^{10}$ It suffices to let $\mu^{n}>A^{n}\left|U_{\max }^{n}-U_{\min }^{n}\right|$, where $U_{\max }^{n}$ and $U_{\min }^{n}$ represent the upper and lower bounds on the payoff function $U^{n}(\cdot)$ for agent $n$, respectively.
} 
Algorithm 5 Local Adaptation and Learning for Social Players

Let $\mathbf{1}_{N}=[1, \cdots, 1]^{T}$ denote an $N \times 1$ column vector of ones, $U_{\max }^{n}$ and $U_{\min }^{n}$ represent the upper and lower bounds on the payoff function $U^{n}(\cdot)$ for agent $n$, respectively, and $I_{\{\cdot\}}$ denote the indicator function.

Initialization: Set $\mu^{n}>A^{n}\left|U_{\max }^{n}-U_{\min }^{n}\right|, \mathbf{p}_{0}^{n}=\frac{1}{A^{n}} \cdot \mathbf{1}_{A^{n}}$, and $R_{0}^{n}=\mathbf{0}$.

Step 1: Action Selection. Choose $\mathrm{a}_{k}^{n} \sim \mathbf{p}_{k}^{n}$, where

$$
p_{k}^{n}(i)= \begin{cases}\frac{1}{\mu^{n}}\left|r_{k}^{n}\left(\mathrm{a}_{k-1}^{n}, i\right)\right|^{+}, & i \neq \mathrm{a}_{k-1}^{n} \\ 1-\sum_{j \neq i} p_{k}^{n}(j), & i=\mathrm{a}_{k-1}^{n}\end{cases}
$$

where $|x|^{+}=\max \{0, x\}$.

Step 2: Information Exchange. Share decisions $\mathrm{a}_{k}^{n}$ with others.

Step 3: Regret Update.

$$
R_{k+1}^{n}=R_{k}^{n}+\varepsilon\left[B^{n}\left(\mathbf{a}_{k}\right)-R_{k}^{n}\right]
$$

where $B^{n}\left(\mathbf{a}_{k}\right)=\left[b_{i j}^{n}\left(\mathbf{a}_{k}\right)\right]$ is an $A^{n} \times A^{n}$ matrix with elements

$$
b_{i j}^{n}\left(\mathbf{a}_{k}\right)=\left[U^{n}\left(j, \mathbf{a}_{k}^{-n}\right)-U^{n}\left(i, \mathbf{a}_{k}^{-n}\right)\right] \cdot I\left\{\mathbf{a}_{k}^{n}=i\right\} .
$$

Step 4: Recursion. Set $k \leftarrow k+1$, and go Step 1 .

\section{Discussion and Intuition:}

1) Adaptive Behavior: In (5.11), $\varepsilon$ serves as a forgetting factor to foster adaptivity to the evolution of the non-cooperative game parameters or the social network architecture. That is, as agents repeatedly take actions, the effect of the old underlying parameters on their current decisions vanishes; see $\$ 5.5$ for a somewhat detailed treatment of regime switching non-cooperative games.

2) Inertia: The choice of $\mu^{n}$ guarantees that there is always a positive probability of playing the same action as the last period. Therefore, $\mu^{n}$ can be viewed as an "inertia" parameter: A higher $\mu^{n}$ yields switching with lower probabilities. It plays a significant role in breaking away from bad cycles. It is worth emphasizing that the speed of convergence to the correlated equilibria set is closely related to this inertia parameter. 
3) Better-reply vs. Best-reply: In light of the above discussion, the most distinctive feature of the regret-matching procedure, that differentiates it from other works such as [90, 92, 93], is that it implements a better-reply rather than a bestreply strategy 11 . This inertia assigns positive probabilities to any actions that are just better. Indeed, the behavior of a regret-matching decision maker is very far from that of a rational decision maker that makes optimal decisions given his (more or less well-formed) beliefs about the environment. Instead, it resembles the model of a reflex-oriented individual that reinforces decisions with "pleasurable" consequences [119].

(4) Computational Complexity: The computational burden (in terms of calculations per iteration) of the "regret-matching" procedure is small. It does not grow with the number of agents, hence, is scalable. At each iteration, each agent needs to execute two multiplications, two additions, one comparison and two table lookups (assuming random numbers are stored in a table) to calculate the next decision. Therefore, it is suitable for implementation in sensors with limited local computational capability.

(5) Decentralized Adaptive Filtering: As will be seen later in $\$ 5.4$, if every agent follows Algorithm 5, the global behavior converges to the set of correlated equilibria. Theoretically, finding a correlated equilibrium in a game is equivalent to solving a linear feasibility problem; see (5.8). Algorithm 5 is thus an adaptive filtering algorithm for solving this problem, which is quite interesting due to its decentralized nature.

\subsubsection{Case II: Social Groups}

As pointed out above, the "perfect monitoring" assumption is quite restrictive in the emerging large-scale social networks. A natural extension is to devise an adaptive learning procedure that relies on the "neighborhood monitoring" model for exchange of information among agents. That is, once a decision is made by agent $n$, it is only observable by her neighbors $N^{n}$ on the connectivity graph $G$; see Definition 5.4. In fact, agents are oblivious to the existence of other agents except their neighbors.

Social groups are characterized by the coalition of agents that perform the same localized task and interact locally. However, not only does the payoff of each agent depend on her local interaction and contribution to carrying out the

\footnotetext{
${ }^{11}$ This has the additional effect of making the behavior continuous, without need for approximations [117].
} 
local tasks, but also it is affected by the inherent interaction with those outside her social group. In particular, the payoff that each agent receives as the outcome of her decision comprises of two terms: (i) Local payoff, due to local interaction (e.g., performing tasks) within social groups; (ii) Global payoff, due to the implicit strategic global interaction with agents outside social group. More precisely, the payoff function can be expressed as [102]

$$
U^{n}\left(\mathrm{a}^{n}, \mathbf{a}^{-n}\right)=U_{L}^{n}\left(\mathrm{a}^{n}, \mathbf{a}^{N^{n}}\right)+U_{G}^{n}\left(\mathrm{a}^{n}, \mathbf{a}^{S^{n}}\right) .
$$

Here, $S^{n}=\mathcal{N}-N_{c}^{n}$ denotes the set of "non-neighbors" of agent $n$.

Now, suppose the game $\mathcal{G}$, defined in $\$ 5.2 .1$, with payoff function (5.16) is being repeatedly played over time: $k=1,2, \ldots$. At time $k$, each agent $n$ makes a decision $\mathrm{a}_{k}^{n}$ and receives a payof $12 U_{k}^{n}\left(\mathrm{a}_{k}^{n}\right)$. Assume that agents know their local payoff functions; hence, knowing the action profile of neighbors, they are able to evaluate their local stage payoffs. In contrast, even if agents know their global payoff functions, they could not directly compute global payoffs as they do not acquire the action profile of agents outside their social groups. However, they can compute their realized global payoffs by

$$
U_{G, k}^{n}\left(\mathrm{a}_{k}^{n}\right) \stackrel{\text { defn }}{=} U_{k}^{n}\left(\mathrm{a}_{k}^{n}\right)-U_{L}^{n}\left(\mathrm{a}^{n}, \mathbf{a}_{k}^{N^{n}}\right) .
$$

The "revised regret-matching" procedure, depicted in Figure 5.3, is then as follows [102, 119]: The local regret matrix $R_{k}^{L, n}$ is defined as before; that is, each element $r_{k}^{L, n}(j, i)$ records

$$
r_{k}^{L, n}(j, i)=\varepsilon \sum_{\tau \leq k}(1-\varepsilon)^{k-\tau}\left[U_{L}^{n}\left(j, \mathbf{a}_{\tau}^{N^{n}}\right)-U_{L}^{n}\left(\mathbf{a}_{\tau}^{n}, \mathbf{a}_{\tau}^{N^{n}}\right)\right] I\left\{\mathbf{a}_{\tau}^{n}=i\right\} .
$$

However, since agent $n$ knows neither her global payoff function nor the decisions $\mathbf{a}_{k}^{S^{n}}$ of those outside her social group, she is unable to perform the thought experiment to evaluate global payoffs for alternative actions as she does in (5.18). She thus employs an unbiased estimator $R_{k}^{G, n}$ of the global regrets, which relies only on the realizations of global payoffs $\left\{U_{G, k}^{n}\left(\mathrm{a}_{k}^{n}\right)\right\}$. More precisely, each element $r_{k}^{G, n}(j, i)$ can be expressed as follows:

$$
\begin{aligned}
r_{k}^{G, n}(j, i)= & \varepsilon \sum_{\tau \leq k}(1-\varepsilon)^{k-\tau} \\
& \times\left[\frac{p_{\tau}^{n}(i)}{p_{\tau}^{n}(j)} U_{G, \tau}^{n}\left(\mathrm{a}_{\tau}^{n}\right) I\left\{\mathrm{a}_{\tau}^{n}=j\right\}-U_{G, \tau}^{n}\left(\mathrm{a}_{\tau}^{n}\right) I\left\{\mathrm{a}_{\tau}^{n}=i\right\}\right] .
\end{aligned}
$$

\footnotetext{
${ }^{12}$ Note that $U_{k}^{n}(\cdot)=U^{n}\left(\cdot, \mathbf{a}_{k}^{-n}\right)$, i.e., the time-dependency of $U_{k}^{n}(\cdot)$ corresponds to the timevariations of other agents' action profile $\mathbf{a}_{k}^{-n}$.
} 


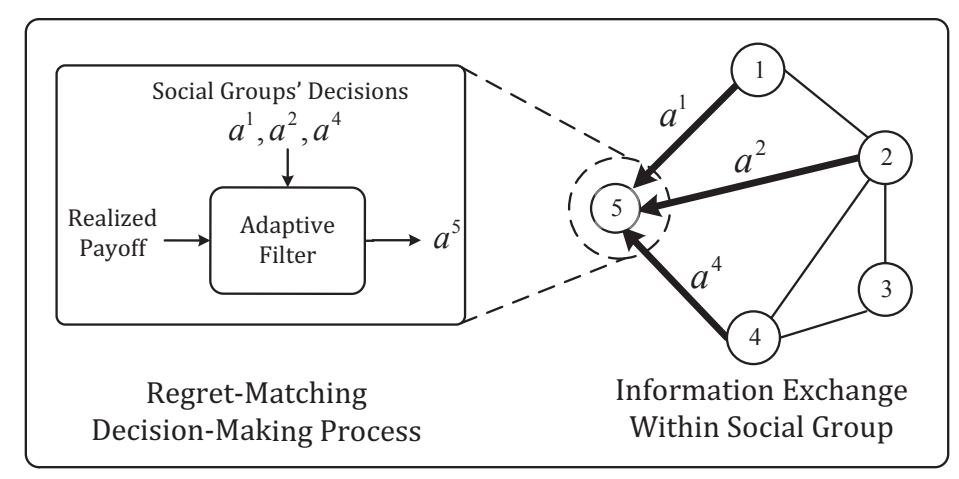

Figure 5.3: Game-theoretic learning within social groups. Circles represent individuals in the social network. Agent 5 exchanges decision with her social group members $N^{5}=\{1,2,4\}$. The exchanged decisions, together with agent 5's realized payoff, are then fed into the regret-matching adaptive filter to determine the next action for agent 5 .

The agent combines local and global regrets

$$
R_{k}^{n} \stackrel{\text { defn }}{=} R_{k}^{L, n}+R_{k}^{G, n}
$$

to update her strategy $\mathbf{p}_{k}^{n}$ as follows: Let $0<\delta<1$, and recall $A^{n}$ represents the cardinality of the action set $\mathcal{A}^{n}$. The play probabilities are then given by

$$
\begin{aligned}
p_{k}^{n}(i) & =\mathbb{P}\left(\mathrm{a}_{k}^{n}=i \mid \mathrm{a}_{k-1}^{n}=j\right) \\
& = \begin{cases}(1-\delta) \min \left\{\frac{1}{\mu^{n}}\left|r_{k}^{n}(j, i)\right|^{+}, \frac{1}{A^{n}}\right\}+\frac{\delta}{A^{n}}, & i \neq j \\
1-\sum_{j \neq i} p_{k}^{n}(j), & i=j\end{cases}
\end{aligned}
$$

where, as before, $|x|^{+}=\max \{0, x\}$, and $\mu^{n}>0$ is a large enough number.

The local adaptation and learning algorithm within social groups is summarized in Algorithm 6 , 


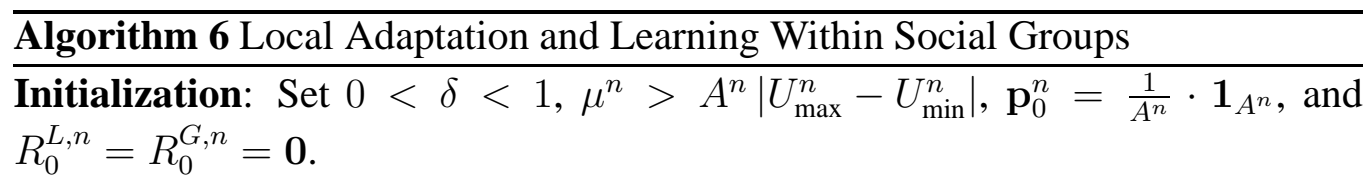

Step 1: Action Selection. Choose $\mathrm{a}_{k}^{n}$ according to the mixed strategy $\mathbf{p}_{k}^{n}$ :

$$
p_{k}^{n}(i)= \begin{cases}(1-\delta) \min \left\{\frac{1}{\mu^{n}}\left|r_{k}^{n}\left(\mathrm{a}_{k-1}^{n}, i\right)\right|^{+}, \frac{1}{A^{n}}\right\}+\frac{\delta}{A^{n}}, & i \neq \mathrm{a}_{k-1}^{n} \\ 1-\sum_{j \neq i} p_{k}^{n}(j), & i=\mathrm{a}_{k-1}^{n}\end{cases}
$$

where $|x|^{+}=\max \{0, x\}$.

Step 2: Information Exchange. Share decisions a $a_{k}^{n}$ with neighbors $N^{n}$. Step 3: Regret Update.

(i) For $m \in\{L, G\}$ :

$$
R_{k+1}^{m, n}=R_{k}^{m, n}+\varepsilon\left[B^{m, n}\left(\mathbf{a}_{k}\right)-R_{k}^{m, n}\right]
$$

where $B^{m, n}\left(\mathbf{a}_{k}\right)=\left[b_{i j}^{m, n}\left(\mathbf{a}_{k}\right)\right]$ is an $A^{n} \times A^{n}$ matrix:

$$
\begin{aligned}
& b_{i j}^{L, n}\left(\mathbf{a}_{k}\right)=\left[U_{L}^{n}\left(j, \mathbf{a}^{-n}\right)-U_{L}^{n}\left(i, \mathbf{a}^{-n}\right)\right] I\left\{\mathbf{a}_{k}^{n}=i\right\} \\
& b_{i j}^{G, n}\left(\mathbf{a}_{k}\right)=\left[\frac{p_{k}^{n}(i)}{p_{k}^{n}(j)} U_{G, k}^{n}\left(\mathbf{a}_{k}^{n}\right) I\left\{\mathbf{a}_{k}^{n}=j\right\}-U_{G, k}^{n}\left(\mathbf{a}_{k}^{n}\right) I\left\{\mathbf{a}_{k}^{n}=i\right\}\right]
\end{aligned}
$$

(ii) Combine local and global regrets:

$$
R_{k}^{n}=R_{k}^{L, n}+R_{k}^{G, n}
$$

Step 4: Recursion. Set $k \leftarrow k+1$, and go Step 1 .

\section{Discussion and Intuition:}

Before proceeding, we should emphasize that the remarks on the "regret-matching" procedure [119], discussed in $\$ 5.3 .1$, will continue to hold for the revised regretmatching algorithm in the presence of social groups.

(1) Global Regret Estimate: Close scrutiny of (5.19) reveals that the second term is similar to the second term in (5.18), and simply evaluates the weighted average global payoffs realized in periods when action $i$ was picked. The first term, roughly speaking, estimates the weighted average global payoffs if action $j$ 
replaced action $i$ in exactly those periods when action $i$ was chosen 13 . To this end, it only uses realized payoffs of those periods when action $j$ was actually played. The normalization factor $p_{\tau}^{n}(i) / p_{\tau}^{n}(j)$, intuitively speaking, makes the lengths of the respective periods comparable [119].

(2) Play Probabilities: The randomized strategy (5.21) is simply a weighted average of two probability vectors: The first term, with weight $1-\delta$, is proportional to the positive part of the combined regrets $\left|R_{k}^{L, n}+R_{k}^{G, n}\right|^{+}$in a manner similar to (5.12). Taking the minimum with $\frac{1}{A^{n}}$ guarantees that $\mathbf{p}_{k}^{n}$ is a valid probability distribution, i.e., $\sum_{i} p_{k}^{n}(i)=1$. The second term, with weight $\delta$, is just a uniform distribution over the action space $\mathcal{A}^{n}$ [102, 119].

(3) Reinforcement Learning: Assuming that no player is willing to form a social group, Algorithm 6 simply implements a reinforcement learning procedure that only requires the realized payoff to prescribe the next decision. The probability of choosing a particular action $i$ at time $k+1$ gets "reinforced" by picking $i$ at time $k$, while the probabilities of the other actions $j \neq i$ decrease. In fact, the higher the payoff realized as the consequence of choosing $i$ at time $k$, the greater will be this reinforcement [119].

(4) Exploration vs. Exploitation: The second term, with weight $\delta$, simply forces every action to be played with some minimal frequency (strictly speaking, with probability $\delta / A^{n}$ ). The "exploration" factor $14 \delta$ is essential to be able to estimate the contingent payoffs using only the realized payoffs; it can, as well, be interpreted as exogenous statistical "noise."

\subsubsection{Case III: Homogeneous Social Groups}

Next, we consider non-cooperative games in the presence of social groups with closer and more intense ties: Individuals in each social group have identical interests in the sense that they all share, and are aware of sharing, the same payoff function. This opens up opportunity for agents to collaborate within social groups to optimize their payoffs, while retaining their autonomy [100].

More precisely, agents form $S$ non-overlapping communities $C^{s} \subset \mathcal{N}, s \in$

\footnotetext{
${ }^{13}$ Strictly speaking, the limit sets of the process $(5.19)$ and the true global regret updates (if agents observed the action profile of agents outside their social group) coincide; see [102] for details. Alternatively, the conditional expectation of the difference between the global regret estimates and the true global regrets can be proved to be zero; see [119] for details.

${ }^{14}$ As will be discussed later, larger $\delta$ will lead to the convergence of the global behavior to a larger $\epsilon$-distance of the correlated equilibria set.
} 
$\mathcal{S}=\{1, \cdots, S\}$, each with cardinality $J_{s}$ and identical payoff functions 15 , i.e.,

$$
U^{n}(\cdot)=U^{l}(\cdot) \Leftrightarrow n, l \in C^{s} \text { for some } s \in \mathcal{S} .
$$

Accordingly, agents have to take actions from the same action set within each community, i.e.,

$$
\mathcal{A}^{n}=\mathcal{A}^{l}(\cdot) \Leftrightarrow n, l \in C^{s} \text { for some } s \in \mathcal{S} \text {. }
$$

For notational convenience, denote by $U^{s}(\cdot)$ and $\mathcal{A}^{s}$ the payoff function and action set, respectively, of community $C^{s}$. Let further $A^{s}=\left|\mathcal{A}^{s}\right|$, where $|\cdot|$ denotes the cardinality operator.

Within communities, agents form social groups. For notational convenience, we continue to use the connectivity graph $G$ in Definition 5.4 to represent such "homogeneous social groups." Individuals in a social group are more closely tied in the sense that they have identical interests and are willing to share the extra (and strategically of great value) piece of information that they share the same payoff function 16 . In particular,

$$
(n, l) \in E \text { in graph } G \Leftrightarrow \text { agents } n \text { and } l \text { both know } U^{n}(\cdot)=U^{l}(\cdot) .
$$

The social group for each agent $n$ is represented by the set of neighbors $N_{c}^{n}$ on the connectivity graph $G$; see (5.9). Finally, for clarity of exposition and without loss of generality, we adopt the "perfect monitoring" [118] model for information exchange among agents; see (5.10). It is straightforward for the interested reader to extend the local adaptation and learning algorithm that follows to the case of "neighborhood monitoring," which was discussed in $\$ 5.3 .2$.

Example 5.1. Consider the problem of multi-target tracking using a network of ZigBee-enabled sensors [161]. Sensors close to each target share the same goal, i.e., to localize the same target, hence, have identical payoff functions. The entire network thus incorporates several communities of sensors with identical payoff functions, each localizing a particular target. The measurements collected by such communities of sensors are correlated both in time and space. Therefore, nodes can save battery power by choosing to sleep while their neighbors are active. Two

\footnotetext{
${ }^{15}$ Without loss of generality, agents belonging to the same community are assumed to have identical action sets.

${ }^{16}$ Note that, although individuals within the same social group are mindful of sharing the same payoff function, they are unaware of the strategies according to which group members make their decisions.
} 
advantages directly follow by letting such sensors become social and exchange information within the social groups that they form: (i) Sharing regrets will lead to faster coordination of their activation behavior; (ii) Sharing local estimates will lead to improved localization performance.

In what follows, inspired by the idea of diffusion least mean squares [182, 225], we enforce cooperation among individuals in each social group via exchanging and fusing regret information. Such diffusion of regret information within social groups is rewarding for all members as they all share the same payoff function. This provably leads to faster coordination among agents and enables them to respond in real time to changes in the game.

The regret matrix $R^{n}$ is defined as in $\$ 5.3 .1$, see (5.11). The cooperative diffusion protocol is then implemented as follows: Agents exchange regret information within social groups and fuse the collected data via a linear combiner. More precisely, define the weight matrix for each community $C^{s}$ as $W^{s}=\left[w_{n l}^{s}\right]$ satisfying

$$
W^{s}=I_{J_{s}}+\mu Q^{s}, \quad 0<\mu<1
$$

where $I_{N}$ denotes the $N \times N$ identity matrix, the matrix $Q^{s}=\left[q_{n l}^{s}\right]$ is symmetric, i.e., $\left[Q^{s}\right]^{\prime}=Q^{s}$ where' denotes the transpose operator, and satisfies

(i) $\left|q_{n l}^{s}\right| \leq 1$;

(ii) $q_{n l}^{s}>0$ if and only if $(k, l) \in E$;

(iii) $Q^{s} \mathbf{1}=\mathbf{0}$, where $\mathbf{1}$ and $\mathbf{0}$ are column vectors of ones and zeros, respectively.

Each row $n$ of the matrix $W^{s}$ simply gives the weights that agent $n$ assigns to the regret information collected from neighbors. These weights may depend on the reputation of the group members [95, 205, 265]. The fused regret matrix for agent $n$ at time $k$, denoted by $\bar{R}_{k}^{n}$, is then computed via

$$
\bar{R}_{k}^{n}=\sum_{l \in N_{c}^{n}} w_{n l}^{s} R_{k}^{l}
$$

Recall that all neighbors of agent $N_{c}^{n}$ belong to the same community as agent $n$. Therefore, they all share the same payoff function. The fused regrets are then promptly fed back into the stochastic approximation algorithm that updates regrets:

$$
R_{k+1}^{n}=\bar{R}_{k}^{n}+\varepsilon\left[B^{n}\left(\mathbf{a}_{k}\right)-\bar{R}_{k}^{n}\right]
$$


where $B^{n}\left(\mathbf{a}_{k}\right)$ is a $N \times N$ matrix with elements identical to (5.15). Finally, agent $n$ picks her next action according to the same strategy $\mathbf{p}^{n}$ as in (5.12). Simply put, agent $n$ follows the "regret-matching" procedure [119], summarized in Algorithm 5, with the exception that fused-regret matrix $\bar{R}_{k}^{n}$ replaces the regret matrix $R_{k}^{n}$.

The cooperative regret-matching procedure in the presence of homogeneous social groups is summarized in Algorithm 7

\section{Discussion and Intuition:}

Before proceeding, we should emphasize that the remarks on the "regret-matching" procedure [119], discussed in $\$ 5.3 .1$, will continue to hold for the revised regretmatching algorithm in the presence of homogeneous social groups.

(1) Diffusion of Regrets: Within each community of agents, the social group that an agent forms may differ from that of his neighbor's. Therefore, the cooperative diffusion protocol (5.30) helps fuse information across the community into agent $n$.

(2) Data Fusion Timescale: The particular choice of the weight matrix $W^{s}$ allows to perform regret update and fusion of social group's information on the same timescale by choosing $\mu=\varepsilon$ in (5.29). This enables the agents to respond in real-time to the evolution of the game, e.g., changes in community memberships or payoffs of communities.

\subsection{Emergence of Rational Global Behavior}

This section characterizes the global behavior emergent from individuals following the local adaption and learning algorithms in $\$ 5.3$. Section 5.4.1 provides a formal definition for the global behavior of agents. Section 5.4.2 then entails the main theorem of this chapter that shows the global behavior manifested as the consequence of each agent individually following algorithms in $\$ 5.3$ converges to the correlated equilibria set. Finally, \$5.4.3 lays out a less technically involved sketch of the convergence proofs that will further shed light on the dynamics of the "regret-matching" procedure and how it could be adapted to strategic decision making in social networks. Detailed proofs are relegated to Appendix B for clarity of presentation. 
Algorithm 7 Adaptation and Learning Within Homogeneous Social Groups Initialization: Set $\mu^{n}>A^{n}\left|U_{\max }^{n}-U_{\min }^{n}\right|, \mathbf{p}_{0}^{n}=\frac{1}{A^{n}} \cdot \mathbf{1}_{A^{n}}$, and $R_{0}^{n}=\mathbf{0}$.

Step 1: Action Selection. Choose $\mathrm{a}_{k}^{n}$ according to the mixed strategy $\mathbf{p}_{k}^{n}$ :

$$
p_{k}^{n}(i)= \begin{cases}\frac{1}{\mu^{n}}\left|r_{k}^{n}\left(\mathrm{a}_{k-1}^{n}, i\right)\right|^{+}, & i \neq \mathrm{a}_{k-1}^{n} \\ 1-\sum_{j \neq i} p_{k}^{n}(j), & i=\mathrm{a}_{k-1}^{n}\end{cases}
$$

where $|x|^{+}=\max \{0, x\}$.

Step 2: Information Exchange. Share decisions $a^{n} k$ with others.

Step 3: Regret Update.

$$
R_{k+1}^{n}=\bar{R}_{k}^{n}+\varepsilon\left[B^{n}\left(\mathbf{a}_{k}\right)-\bar{R}_{k}^{n}\right]
$$

where $B^{n}\left(\mathbf{a}_{k}\right)$ is an $A^{s} \times A^{s}$ matrix identical to Algorithm 5 ,

(The first two steps implement the regret-matching procedure [117].)

\section{Step 4: Regret Fusion.}

$$
\bar{R}_{k+1}^{n}=\bar{R}_{k}^{n}+\mu\left[D^{n}\left(\mathbf{a}_{k}\right)-\bar{R}_{k}^{n}\right]
$$

where $D^{n}\left(\mathbf{a}_{k}\right)=\left[d_{i j}^{n}\left(\mathbf{a}_{k}\right)\right]$ is an $A^{s} \times A^{s}$ matrix:

$$
d_{i j}^{n}\left(\mathbf{a}_{k}\right)=\sum_{l \in \mathcal{N}_{c}^{n}} w_{n l}^{s}\left[U^{s}\left(j, \mathbf{a}_{k}^{-l}\right)-U^{s}\left(i, \mathbf{a}_{k}^{-l}\right)\right] I\left\{\mathbf{a}_{k}^{l}=i\right\} .
$$

and $s$ denotes the index of the community to which agent $n$ belongs.

(This step implements the diffusion protocol [182 225].)

Step 5: Recursion. Set $k \leftarrow k+1$, and go Step 1 . 


\subsubsection{Global Behavior}

The global behavior $\boldsymbol{z}_{k}$ at time $k$ is defined as the empirical frequency of joint play of all agents up to period $k$. Formally,

$$
\boldsymbol{z}_{k}=\sum_{\tau \leq k}(1-\varepsilon)^{k-\tau} \boldsymbol{e}_{\mathbf{a}_{\tau}}
$$

where $e_{\mathbf{a}_{\tau}}$ denotes the unit vector with the element corresponding to the joint play $\mathbf{a}_{\tau}$ being equal to one. Further, $\varepsilon$ serves as a forgetting factor to foster adaptivity to the evolution of game. That is, the effect of the old game model on the decisions of individuals vanishes as agents repeatedly take actions. Given $\boldsymbol{z}_{k}$, the average payoff accrued by each agent can be straightforwardly evaluated, hence the name global behavior. It is more convenient to define $\boldsymbol{z}_{k}$ via the stochastic approximation recursion

$$
\boldsymbol{z}_{k}=\boldsymbol{z}_{k-1}+\varepsilon\left[\boldsymbol{e}_{\mathbf{a}_{k}}-\boldsymbol{z}_{k-1}\right]
$$

The global behavior $z_{k}$ is a system "diagnostic" and is only used for the analysis of the emergent collective behavior of agents. That is, it does not need to be computed by individual agents. In real-life application such as smart sensor networks, however, a network controller can monitor $\boldsymbol{z}_{k}$ and use it to adjust agents' payoff functions to achieve the desired global behavior [191].

\subsubsection{Main Result: Convergence to Correlated Equilibrium}

In what follows, the main theorem of this chapter is presented that reveals both the local and the global behavior emerging from each agent individually following the algorithms presented in $\$ 5.3$. We use $R_{k}^{n}$ and $\boldsymbol{z}_{k}$ as indicative of agent's local and global experience, respectively.

The main theorem simply asserts that, if an agent individually follows any of the algorithms of $\$ 5.3$ (depending on the particular game and connectivity graph model), she will experience regret of at most $\epsilon$ (for a small number $\epsilon$ ) after sufficient repeated plays of the game. Indeed, the number $\epsilon$ diminishes and asymptotically goes to zero in Algorithms 5]and 7, where agents exhibit no exploration. This theorem further states that if now all agents start following any of algorithms of $\$ 5.3$ independently, the global behavior converges to the correlated equilibria set. Differently put, agents can coordinate their strategies in a distributed fashion so that the distribution of their joint behavior belongs to the correlated equilibria 
set. From the game-theoretic point of view, we show non-fully rational local behavior of individuals, due to utilizing a "better-reply" rather than a "best-reply" strategy, can lead to the manifestation of globally sophisticated behavior.

Before proceeding with the theorem, recall that weak convergence is a generalization of convergence in distribution to a function space 17 ; see Definition 3.2 in $\$ 3.5 .2$.

Theorem 5.4.1. Suppose the game $\mathcal{G}$, defined in $\$ 5.2 .1$ is being repeatedly played. Define the continuous-time interpolated sequence of the global behavior iterates $\boldsymbol{z}_{k}$ as follows:

$$
\boldsymbol{z}^{\varepsilon}(t)=\boldsymbol{z}_{k} \quad \text { for } \quad t \in[k \varepsilon,(k+1) \varepsilon)
$$

where $\boldsymbol{z}_{k}$ is defined in (5.36). With a slight abuse of notation, denote by $R_{k}^{n}$ the regret matrices rearranged as a vector of length $\left(A^{n}\right)^{2}$, and define the continuoustime interpolated sequence

$$
R^{n, \varepsilon}(t)=R_{k}^{n} \quad \text { for } \quad t \in[k \varepsilon,(k+1) \varepsilon)
$$

where $R_{k}^{n}$ is given in (5.11) and (5.20). Let further $\mathbb{R}^{-}$represent the negative orthant and $\|\cdot\|$ denote the Euclidean norm. Then, the following results hold:

Result 1: Consider "Case I" that examines fully social players; see 5.3 .1 If every agent follows the "regret-matching" procedure [117], summarized in Algorithm 5 then as $\varepsilon \rightarrow 0$ and $t \rightarrow \infty$ :

(i) $R^{n, \varepsilon}(t)$ converges weakly to the negative orthant in the sense that

$$
\operatorname{dist}\left[R^{n, \varepsilon}(t), \mathbb{R}^{-}\right]=\inf _{\boldsymbol{r} \in \mathbb{R}^{-}}\left\|R^{n, \varepsilon}(t)-\boldsymbol{r}\right\| \Rightarrow 0
$$

(ii) $\boldsymbol{z}^{\varepsilon}(t)$ converges weakly to the correlated equilibria set $\mathcal{C}$ in the sense that

$$
\operatorname{dist}\left[\boldsymbol{z}^{\varepsilon}(t), \mathcal{C}\right]=\inf _{\boldsymbol{z} \in \mathcal{C}}\left\|\boldsymbol{z}^{\varepsilon}(t)-\boldsymbol{z}\right\| \Rightarrow 0
$$

Result 2: Consider "Case II" that examines formation of social groups; see $\$ 5.3 .2$ For each $\epsilon$, there exists an upper bound $\widehat{\delta}(\epsilon)$ on the exploration factor $\delta$ such that, if every agent follows Algorithm 6 with $\delta<\widehat{\delta}(\epsilon)$, as $\varepsilon \rightarrow 0$ and $t \rightarrow \infty$ :

\footnotetext{
${ }^{17}$ We refer the interested reader to [167, Chapter 7] for further details on weak convergence and related matters.
} 
(i) $R^{n, \varepsilon}(t)$ converges weakly to $\epsilon$-distance of the negative orthant, i.e.,

$$
\operatorname{dist}\left[R^{n, \varepsilon}(t), \mathbb{R}^{-}\right]-\epsilon \Rightarrow 0
$$

(ii) $\boldsymbol{z}^{\varepsilon}(t)$ converges weakly to the correlated $\epsilon$-equilibria set $\mathcal{C}_{\epsilon}$, i.e.,

$$
\operatorname{dist}\left[\boldsymbol{z}^{\varepsilon}(t), \mathcal{C}_{\epsilon}\right] \Rightarrow 0
$$

Result 3: Consider "Case III" that examines formation of homogeneous social groups; see §5.3.3. If every agent follows Algorithm 7 then as $\varepsilon \rightarrow 0$ and $t \rightarrow \infty$ :

(i) $R^{n, \varepsilon}(t)$ converges weakly to the negative orthant.

(ii) $\boldsymbol{z}^{\varepsilon}(t)$ converges weakly to the correlated equilibria set $\mathcal{C}$.

\section{Discussion:}

1) Convergence to Set: Note that Theorem 5.4.1 proves convergence of $\boldsymbol{z}_{k}$ to the correlated equilibria set $\mathcal{C}$, rather than a point in the set $\mathcal{C}$. In fact, once the convergence occurs, $\boldsymbol{z}_{k}$ can generally move around in the polytope $\mathcal{C}$. The same argument holds for convergence of regrets $R_{k}^{n}$ to the negative orthant.

2) Time-invariant Games: If the parameters of the game $\mathcal{G}$ (such as the payoff functions, action sets, etc.) do not evolve with time, one can replace the constant adaptation rate $\varepsilon$ with the decreasing step-size $\varepsilon_{k}=1 / k$ in Algorithms 5-7. This results in achieving stronger convergence results in the sense that one can now prove almost sure convergence to the correlated equilibria set. More precisely, there exists with probability one $K(\epsilon)>0$ such that for $k>K(\epsilon)$, one can find a correlated equilibrium joint distribution $\pi \in \mathcal{C}$ at most at $\epsilon$-distance of $\boldsymbol{z}_{k}$ [117].

\subsubsection{Sketch of Convergence Proof}

Let us now proceed with a sketch of the proof of the main results summarized in Theorem 5.4.1. For better readability, details for each step of the proof are postponed to Appendix B. Certain technical details, that are out of the scope of this chapter, are also passed over; however, adequate citation will be provided for the interested reader. The convergence analysis is based on [26] and is organized into three steps as follows: First, it will be shown that the limit systems for the discrete time iterates of the presented algorithms are differential inclusions 18 . Differential

\footnotetext{
${ }^{18}$ Differential inclusions are generalization of the concept of ordinary differential equation. A generic differential inclusion is of the form $d X / d t \in \mathcal{F}(X, t)$, where $\mathcal{F}(X, t)$ specifies a family of trajectories rather than a single trajectory as in the ordinary differential equations $d X / d t=$ $F(X, t)$. See Appendix $\mathrm{B}$ for a formal definition.
} 
inclusions arise naturally in game-theoretic learning, since the strategies according to which others play are unknown. Next, using Lyapunov function methods, stability of the limit dynamical system is studied and its set of global attractors is characterized. Accordingly, asymptotic stability of the interpolated processes associated with the discrete-time iterates is proved. Up to this point, we have shown that each agent asymptotically experiences zero (or at most $\epsilon$ ) regret by following any of the algorithms presented in $\$ 5.3$. In the final step, it will be shown that the global behavior emergent from such limit individual dynamics in the game is attracted to the correlated equilibria set.

For the sake of simplicity of exposition, we shall focus in what follows on the proof for the "regret-matching" procedure (Algorithm 5). We then elaborate on how to modify each step of the proof so as to adapt it to the assumptions made in "Case II" and "Case III" in $\$ 5.3$.

Before proceeding with the proof, we shall study properties of the sequence of decisions $\left\{\mathrm{a}_{k}^{n}\right\}$ made according to Algorithms 5-7, Careful consideration of the strategy $\mathbf{p}_{k}^{n}$ in the "regret-matching" procedure indicates that the sequence of decisions $\left\{\mathrm{a}_{k}^{n}\right\}$ simply forms a finite-state Markov chain. Standard results on Markov chains show that the transition probabilities in (5.12) admits (at least) one invariant measure. Let $\psi^{n}\left(R^{n}\right)$ denote such an invariant measure. Then, the following lemma holds.

Lemma 5.1. The invariant measure $\psi^{n}\left(R^{n}\right)$ satisfies 19

$$
\sum_{i \neq j} \psi_{i}^{n}\left(R^{n}\right)\left|r^{n}(i, j)\right|^{+}=\sum_{i \neq j} \psi_{j}^{n}\left(R^{n}\right)\left|r^{n}(j, i)\right|^{+} .
$$

Proof. Letting $\psi^{n}\left(R^{n}\right)$ denote the invariant measure of transition probabilities (15.12), one can write

$$
\psi_{i}^{n}\left(R^{n}\right)=\psi_{i}^{n}\left(R^{n}\right)\left[1-\sum_{j \neq i} \frac{\left|r^{n}(j, i)\right|^{+}}{\mu^{n}}\right]+\sum_{j \neq i} \psi_{j}^{n} \cdot \frac{\left|r^{n}(i, j)\right|^{+}}{\mu^{n}} .
$$

Cancelling out $\psi_{i}^{n}\left(R^{n}\right)$ on both sides and rearranging the equality yields the desire result.

We shall now proceed with the proof.

\footnotetext{
${ }^{19}$ Equation (5.42) together with $\psi^{n}\left(R^{n}\right) \geq 0$ and $\sum_{i} \psi_{i}^{n}\left(R^{n}\right)=1$ forms a linear feasibility problem (null objective function). Existence of $\psi^{n}\left(R^{n}\right)$ can alternatively be proved using strong duality; see [37, Sec. 5.8.2] for details.
} 


\section{Step 1: Characterization of Limit Individual Behavior}

The first step characterizes the asymptotic behavior of individual agents as a differential inclusion when they follow algorithms of $\$ 5.3$. We borrow techniques from the theory of stochastic approximations [167] and work with the piecewiseconstant continuous-time interpolations of the discrete-time iterates of regrets. The regret matrix $R_{k}^{n}$ incorporates all information recorded in $\boldsymbol{z}_{k}$, however, translated to each agent's language based on her payoff function. Since it is more convenient to work with $R_{k}^{n}$, we characterize the limiting process for $R_{k}^{n}$ and analyze its stability instead in the next step. Technical details are tedious and are only provided for "Case I". The interested reader is referred to [102], [167, Chapter 8] for more details.

Case I: Let $\mathrm{p}^{-n}$ denote a probability measure over the joint action space $\mathcal{A}^{-n}$ of all agents excluding agent $n$, and denote by $\Delta \mathcal{A}^{-n}$ the simplex of all such measures. The expected payoff to agent $n$ given the joint strategy $\mathbf{p}^{-n}$ is then defined as

$$
U^{n}\left(\mathbf{a}^{n}, \mathbf{p}^{-n}\right) \stackrel{\text { defn }}{=} \sum_{\mathbf{a}^{-n}} p^{-n}\left(\mathbf{a}^{-n}\right) U^{n}\left(\mathbf{a}^{n}, \mathbf{a}^{-n}\right)
$$

where $p^{-n}\left(\mathbf{a}^{-n}\right)$ denotes the probability of all agents excluding agent $n$ jointly picking $\mathbf{a}^{-n}$.

Theorem 5.4.2. As $\varepsilon \rightarrow 0$, the interpolated process $R^{n, \varepsilon}(\cdot)$ converges weakly to $R^{n}(\cdot)$, that is a solution to the differential inclusion 20

$$
\frac{d R^{n}}{d t} \in H^{n}\left(R^{n}\right)-R^{n}
$$

where $H^{n}\left(R^{n}\right)$ is an $A^{n} \times A^{n}$ matrix with elements defined below:

$$
h_{i j}^{n}\left(R^{n}\right) \stackrel{\text { defn }}{=}\left\{\left[U^{n}\left(j, \mathbf{p}^{-n}\right)-U^{n}\left(i, \mathbf{p}^{-n}\right)\right] \psi_{i}^{n}\left(R^{n}\right) ; \mathbf{p}^{-n} \in \Delta \mathcal{A}^{-n}\right\} .
$$

Proof. See Appendix B.1.

Discussion and Intuition: The asymptotics of a stochastic approximation algorithm is typically captured by an ordinary differential equation (ODE). Here, although agents observe $\mathbf{a}^{-n}$, they are oblivious to the strategies $\mathbf{p}^{-n}$ from which $\mathbf{a}^{-n}$ has been drawn. Different $\mathbf{p}^{-n} \in \Delta \mathcal{A}^{-n}$ form different trajectories of $R^{n}(t)$.

\footnotetext{
${ }^{20}$ Since the r.h.s. in (5.44) is a compact convex set, the linear growth condition (B.2) for the differential inclusion is trivially satisfied; see Definition B.1 for detials.
} 
Therefore, $R^{n, \varepsilon}(t)$ converges weakly to the trajectories of a differential inclusion rather than an ODE.

Case II: Let $\boldsymbol{\nu}^{-n}$ denote a probability measure over the joint action space of the open neighborhood of agent $n$, and denote by $\Delta \mathcal{A}^{N^{n}}$ the simplex of all such measures. The expected local payoff to agent $n$ given the joint strategy $\nu^{-n}$ can be defined similar to (5.43). It is straightforward to show that the invariant measure of the transition probabilities (5.32) now takes the form

$$
\boldsymbol{\sigma}^{n}\left(R^{n}\right)=(1-\delta) \boldsymbol{\psi}^{n}\left(R^{n}\right)+\frac{1}{A^{n}} \cdot \mathbf{1}_{N}
$$

where $\psi^{n}\left(R^{n}\right)$ is the stationary distribution satisfying (5.42); see Lemma 5.1 . That is, $\boldsymbol{\psi}^{n}\left(R^{n}\right)$ corresponds to the stationary distribution of the transitions probabilities (5.32) when the exploration factor $\delta=0$.

Theorem 5.4.3. Define the continuous-time interpolated sequence

$$
\begin{aligned}
& R^{L, n, \varepsilon}(t)=R_{k}^{L, n} \\
& R^{G, n, \varepsilon}(t)=R_{k}^{G, n}
\end{aligned} \quad \text { for } \quad t \in[k \varepsilon,(k+1) \varepsilon)
$$

where $R_{k}^{L, n}$ and $R_{k}^{G, n}$ are given in $₫ 5.3 .2$. Then, as $\varepsilon \rightarrow 0$, the interpolated pair processes $\left(R^{L, n, \varepsilon}(\cdot), R^{G, n, \varepsilon}(\cdot)\right)$ converges weakly to $\left(R^{L, n}(\cdot), R^{G, n}(\cdot)\right)$, that is a solution to the system of interconnected differential inclusion

$$
\left\{\begin{array}{l}
\frac{d R^{L, n}}{d t} \in H^{L, n}\left(R^{L, n}, R^{G, n}\right)-R^{L, n} \\
\frac{d R^{G, n}}{d t} \in H^{G, n}\left(R^{L, n}, R^{G, n}\right)-R^{G, n} .
\end{array}\right.
$$

where $H^{L, n}\left(R^{L, n}, R^{G, n}\right)$ and $H^{G, n}\left(R^{L, n}, R^{G, n}\right)$ are $A^{n} \times A^{n}$ matrices with elements defined as follows:

$$
\begin{aligned}
& h_{i j}^{L, n} \stackrel{\text { defn }}{=}\left\{\left[U_{L}^{n}\left(j, \boldsymbol{\nu}^{-n}\right)-U_{L}^{n}\left(i, \boldsymbol{\nu}^{-n}\right)\right] \sigma_{i}^{n}\left(R^{n}\right) ; \boldsymbol{\nu}^{-n} \in \Delta \mathcal{A}^{N^{n}}\right\} \\
& h_{i j}^{G, n} \stackrel{\text { defn }}{=}\left[U_{G, t}^{n}(j)-U_{G, t}^{n}(i)\right] \sigma_{i}^{n}\left(R^{n}\right)
\end{aligned}
$$

where $U_{G, t}^{n}(\cdot)$ is the interpolated process of the global payoffs accrued from the game, $\sigma^{n}\left(R^{n}\right)$ is given in (5.46), and $R^{n}=R^{L, n}+R^{G, n}$.

Discussion and Intuition: The interconnection of the dynamics in (5.48) is hidden in $\boldsymbol{\sigma}^{n}\left(R^{n}\right)$ since it is a function of the pair $\left(R^{L, n}, R^{G, n}\right)$; see (5.20). Agents are oblivious to the strategies $\nu^{-n}$ from which the decision $\mathbf{a}^{N^{n}}$ has been 
made. Therefore, following the same argument as in "Case I," $R^{L, n, \varepsilon}(\cdot)$ converges weakly to the trajectories of a differential inclusion. The same argument holds for $R^{G, n, \varepsilon}(\cdot)$ except that the limit dynamics follows an ODE. This is due to agents being oblivious to the facts: (i) non-neighbors exist, and (ii) global payoffs are affected by non-neighbors' decisions. However, they realize the time-dependency of $U_{G, k}^{n}(\cdot)$ as taking the same action at various times results in different payoffs.

Case III: In light of the diffusion protocol, agents' successive decisions affect, not only their own future strategies, but also their social groups' policies. This suggests looking at the dynamics of the regret for the entire social group to account for such collaboration. Accordingly, define

$$
\mathbf{R}_{k}^{s} \stackrel{\text { defn }}{=} \operatorname{col}\left\{R_{k}^{n_{1}}, \ldots, R_{k}^{n_{J_{s}}}\right\}, \quad \forall n_{j} \in C^{s}
$$

and the associated sequence of interpolated process

$$
\mathbf{R}^{s, \varepsilon}(t)=\mathbf{R}_{k}^{s} \text { for } t \in[k \varepsilon,(k+1) \varepsilon) .
$$

Let further $\otimes$ denote the Kronecker product.

Theorem 5.4.4. As $\varepsilon \rightarrow 0$, the interpolated process $\mathbf{R}^{s, \varepsilon}(\cdot)$ converges weakly to $\mathbf{R}^{s}(\cdot)$ that is a solution of the differential inclusion

$$
\frac{d \mathbf{R}^{s}}{d t} \in \mathbf{H}^{s}\left(\mathbf{R}^{s}\right)+\left(\mathbf{Q}^{s}-I\right) \mathbf{R}^{s}
$$

where $\mathbf{Q}^{s}=Q^{s} \otimes I_{A^{s}}$ (see (5.29)), $A^{s}$ denotes the cardinality of the action set of community $C^{s}$, and

$$
\begin{gathered}
\mathbf{h}_{i j}^{s}\left(\mathbf{R}^{s}\right) \stackrel{\text { defn }}{=}\left\{\left[U^{s}\left(\iota, \mathbf{p}^{-\kappa}\right)-U^{s}\left(j, \mathbf{p}^{-\kappa}\right)\right] \psi_{\iota}^{\kappa} ; \mathbf{p}^{-\kappa} \in \Delta \mathcal{A}^{-\kappa}\right\} \\
\iota=i \bmod A^{s}, \kappa=\left\lfloor\frac{i}{A^{s}}\right\rfloor+1 .
\end{gathered}
$$

Further, $\psi^{n}$ represents the stationary distribution of the transition probabilities (5.32), which satisfies (5.42).

\section{Step 2: Stability Analysis of Limit Dynamical System}

This step examines stability of the limit systems that have been shown to represent the local dynamical behavior of agents in Step 1. The set of global attractors of these limit systems are then shown to comprise the negative orthant $\mathbb{R}^{-}$; see 
Figure 5.4a, Therefore, agents asymptotically experience zero (or at most as small as $\epsilon$ ) regret via exhibiting the simple local behavior prescribed by Algorithms $5-7,7$,

In what follows, we unfold the regret matrix and rearrange its elements as a vector. With a slight abuse of notation, we continue to use the same notation for this regret vector.

Theorem 5.4.5. The limit systems presented in Theorems 5.4.2 5.4.4 are globally asymptotically stable. In particular,

$$
\lim _{t \rightarrow \infty} \operatorname{dist}\left[R^{n}(t), \mathbb{R}^{-}\right]=0
$$

where dist $[\cdot, \cdot]$ denotes the usual distance function.

Proof. See Appendix B.

The following corollary then follows from Theorems 5.4.2 -5.4 .4 and Theorem 5.4.5. It asserts that the result of Theorem 5.4.5 also holds for the interpolated processes associated with the regrets - they also converge to the negative orthant; see Figure 5.4a.

Corollary 5.1. Consider the interpolated process $R^{n, \varepsilon}(\cdot)$, defined in (5.39). Then,

$$
\lim _{t \rightarrow \infty} \lim _{\varepsilon \rightarrow 0}\left|R^{n, \varepsilon}(t)\right|^{+}=\mathbf{0}
$$

where $|\cdot|^{+}$denotes the element-wise positive operator, and 0 represents the zero vector of the same size as $R^{n, \varepsilon}(t)$.

\section{Step 3: Convergence to Correlated Equilibria Set}

In the final step of the proof we characterize the global behavior of agents whose local behavior ensures no (or small $\epsilon$ ) asymptotic regret; see Figure 5.4. It will be shown that the regret of individual agents converging to the negative orthant provides the necessary and sufficient condition for convergence of global behavior to the correlated equilibria set.

Theorem 5.4.6. Recall the interpolated process for global behavior $\boldsymbol{z}^{\varepsilon}(\cdot)$, defined in (5.38), and the interpolated regret process $R^{n, \varepsilon}(\cdot)$, defined in (5.39). Then,

$$
\boldsymbol{z}^{\varepsilon}(\cdot) \Rightarrow \mathcal{C} \quad \text { iff } \quad R^{n, \varepsilon}(\cdot) \Rightarrow \mathbb{R}^{-} \text {for all } n \in \mathcal{N}
$$

Proof. The detailed proof is provided in Appendix B.

The above theorem, together with Corollary 5.1, completes the proof. 


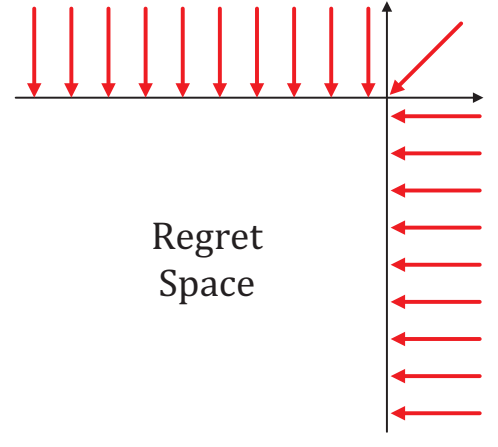

(a)

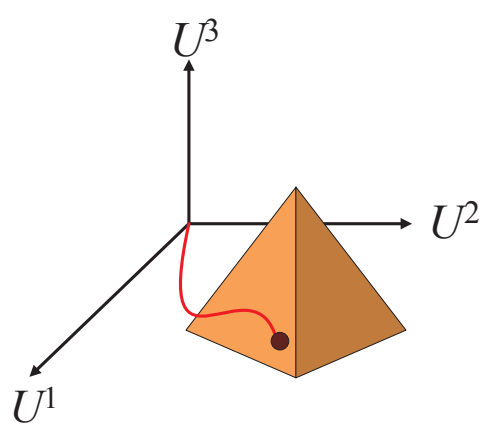

(b)

Figure 5.4: (a) Asymptotic stability and convergence of regrets to the negative orthant (Corollary 5.1). (b) A typical correlated equilibria polytope in a threeplayer game. The red line depicts the trajectory of average payoff accrued by each player (Theorem 5.4.6).

\subsection{Markov Modulated Non-Cooperative Games}

In this section, motivated by applications in cognitive networks [101, 161, 191], we introduce a general class of Markov modulated noncooperative games. We then comment on whether the results of $\$ 5.4$ can be generalized to such settings. In particular, we examine if the simple local adaptation and learning algorithms, presented in $\S 5.3$, can lead to a global behavior that is agile enough to track the regime-switching polytope of correlated equilibria.

Suppose the non-cooperative game model, formulated in $\$ 5.2 .1$, evolves with time due to: (i) agents joining or leaving the game; (ii) changes in agents' incentives (payoffs); and (iii) the connectivity graph $G$ varying with time. Suppose further that all time-varying parameters are finite-state and absorbed to a vector indexed by $\theta$. It is thus reasonable to model evolution of the game via a discretetime finite-state Markov chain $\left\{\theta_{k}\right\}$. Without loss of generality, assume that the Markov chain has state-space $\mathcal{M}=\{1, \ldots, M\}$ and transition probability matrix

$$
P^{\rho}=I_{M}+\rho Q
$$

Here, $\rho>0$ is the small parameter that, roughly speaking, determines the speed of jumps of $\left\{\theta_{k}\right\}, I_{M}$ denotes the $M \times M$ identity matrix, and $Q=\left[q_{i j}\right]$ is the generator of a continuous-time Markov chain satisfying 
(i) $Q \mathbf{1}_{M}=\mathbf{0}_{M}$;

(ii) $q_{i j} \geq 0$ for $i \neq j$;

(iii) $\left|q_{i j}\right| \leq 1$ for all $i, j \in \mathcal{M}$.

Choosing $\rho$ small enough ensures that $p_{i j}^{\rho}=\delta_{i j}+\rho q_{i j}>0$ in the entries of (5.57), where $\delta_{i j}$ denotes the Kronecker $\delta$ function satisfying $\delta_{i j}=1$ if $i=j$ and 0 otherwise.

For better exposition, let us assume that the payoff functions vary with the state of the Markov chain $\left\{\theta_{k}\right\}$ in the rest of this section. More precisely, the payoff function for each agent $n$ takes the form

$$
U^{n}\left(\mathrm{a}^{n}, \mathbf{a}^{-n}, \theta\right): \mathcal{A}^{n} \times \mathcal{A}^{-n} \times \mathcal{M} \rightarrow \mathbb{R} .
$$

The main assumption here is that agents do not observe the Markov chain $\left\{\theta_{k}\right\}$, nor are they aware of its dynamics. Agents however may realize time-dependency of the payoff functions as taking the same action at different time instants may result in different payoffs. Put differently, it does not enter implementation of the local adaptation and learning algorithms. The Markov chain dynamics are used in analyzing the tracking capability of the algorithms.

As the payoff functions jump change according to the Markov chain $\left\{\theta_{k}\right\}$, so does the correlated equilibria set as illustrated in Figure 5.5. We thus need to modify Definition 5.3 to incorporate $\theta$-dependency of the payoff functions as follows:

Definition 5.5. Let $\mathbf{p}$ denote a probability distribution on the space of action profiles $\mathcal{A}$. The set of correlated equilibria of the regime-switching game is the convex polytope

$$
\mathcal{C}_{\epsilon}\left(\theta_{k}\right)=\left\{\mathbf{p}: \sum_{\mathbf{a}^{-n}} p^{n}\left(i, \mathbf{a}^{-n}\right)\left[U^{n}\left(j, \mathbf{a}^{-n}, \theta_{k}\right)-U^{n}\left(i, \mathbf{a}^{-n}, \theta_{k}\right)\right] \leq 0, \forall i, j, n\right\}
$$

where $p^{n}\left(i, \mathbf{a}^{-n}\right)$ denotes the probability of agent $n$ playing action $i$ and the rest playing $\mathbf{a}^{-n}$.

The following theorem then provides a condition that ensures the local adaptation and learning algorithms of $\$ 5.3$ (with no further modifications) generate trajectories of global behavior $z_{k}$ that tracks the regime switching set of correlated equilibrium $\mathcal{C}\left(\theta_{k}\right)$. 


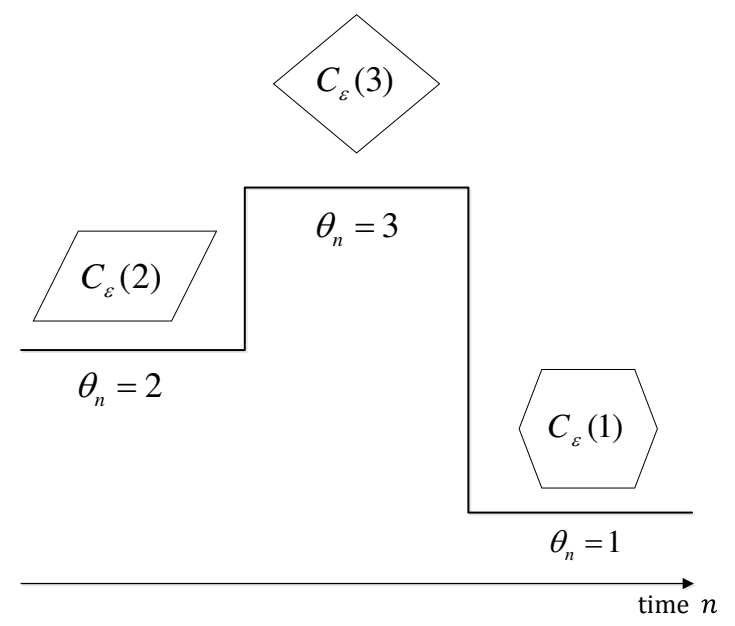

Figure 5.5: Tracking convex polytope of correlated equilibria $\mathcal{C}\left(\theta_{k}\right)$ randomly evolving with time according to a Markov process $\left\{\theta_{k}\right\}$. Theorem 5.5 .1 asserts that such tracking is attainable by each agent individually following the regretmatching algorithms presented in $₫ 5.3$,

Theorem 5.5.1. Consider the non-cooperative game $\mathcal{G}$ regime switching according to a finite-state Markov chain $\left\{\theta_{k}\right\}$ with transition probabilities given by (5.57). Suppose that the step-size of Algrithms $5-7$ is small, i.e., $0<\varepsilon \ll 1$. Assume further that that

$$
\text { the process } \theta_{k} \text { is "slow" in the sense that } \rho=\varepsilon \text {. }
$$

Recall the interpolated sequence of regrets $R^{n, \varepsilon}(\cdot)$, defined in (5.39), and interpolated global behavior $\boldsymbol{z}^{\varepsilon}(\cdot)$, defined in (5.38). If every agent follows Algorithms $5-$ 7 as $\varepsilon \rightarrow 0$ and $t \rightarrow \infty 21$ :

(i) $R^{n, \varepsilon}(t)$ converges weakly to the negative orthant in the sense that

$$
\operatorname{dist}\left[R^{n, \varepsilon}(t), \mathbb{R}^{-}\right]=\inf _{r \in \mathbb{R}^{-}}\left\|R^{n, \varepsilon}(t)-\boldsymbol{r}\right\| \Rightarrow 0 ;
$$

(ii) $\boldsymbol{z}^{\varepsilon}(\cdot)$ converges weakly to the regime-switching polytope of correlated equilibria $\mathcal{C}(\theta(\cdot))$. More precisely,

$$
\operatorname{dist}\left[\boldsymbol{z}^{\varepsilon}(\cdot), \mathcal{C}(\theta(\cdot))\right]=\inf _{\boldsymbol{z} \in \mathcal{C}(\theta(\cdot))}\left|\boldsymbol{z}^{\varepsilon}(\cdot)-\boldsymbol{z}\right| \Rightarrow 0 .
$$

\footnotetext{
${ }^{21}$ To be more precise, the following results hold for an $\epsilon$ neighborhood of the convergent sets in "Case II".
} 
where $\theta(\cdot)$ is a continuous-time Markov chain with generator $Q$; see (5.57).

Proof. We skip the detailed proof for the sake of brevity. Here, we only provide a descriptive sketch of the steps of the proof: First, by a combined use of updated treatment on stochastic approximation [167], and Markov-switched systems [261, [263], we show that the limiting behavior converges weakly to the differential inclusions presented in Theorems 5.4.2 5.4.4 modulated by a continuous-time Markov chain with generator $Q$. Next, using the multiple Lyapunov function method [53, 54], [177, Chapter 3], we show that the limiting dynamical system is globally asymptotically stable almost surely. The interested reader is referred to [102] for the detailed proof in "Case II."

Discussion and Intuition: It is well known that: (i) If the underlying parameter $\theta_{k}$ change too drastically, there is no chance one can track the time-varying properties via an adaptive stochastic approximation algorithm. (Such a phenomenon is known as trackability [29].) b) If the parameters evolve on a slower time-scale as compared to the stochastic approximation algorithm, they remain constant in the fast time-scale and their variation can be asymptotically ignored. Condition (5.59) simply ensures that the parameters of the game model evolve according to a Markov chain that evolves on the same timescale as Algorithms 5, 7, The above theorem then asserts that, if the "matching-speed" condition holds, Algorithms 5 7 are agile in tracking the jump changes in the correlated equilibria set. From the game-theoretic point of view, non-fully-rational behavior of individual agents (due to utilizing a "better-reply" rather than a "best-reply" strategy) leads to a sophisticated globally rational behavior that can adapt to time inhomogeneities in the game model.

\subsection{Example: Energy-Aware Sensing via Diffusion LMS}

This section provides an example of how the adaptation and learning algorithms of $\$ 5.3$ can be applied to achieve sophisticated global behavior in a social sensing application. We consider energy-aware activation control of sensors aiming to collaboratively estimate the true value of a common parameter of interest 22 . We focus on the diffusion least mean square (LMS) algorithms [182, 224, 226];

\footnotetext{
${ }^{22}$ The material in this section is based on the recent paper [101].
} 
however, the proposed framework can applied to any collaborative non-Bayesian estimation setting.

\subsubsection{Centralized Parameter Estimation Problem}

Consider a set of $N$ sensors $\mathcal{N}=\{1, \ldots, N\}$ with the objective to estimate an unknown $M \times 1$ parameter vector $\psi^{o}$ via recording noisy measurements

$$
y_{k}^{n}=\boldsymbol{u}_{k}^{n} \boldsymbol{\psi}^{o}+v_{k}^{n}, \quad n=1, \ldots, N .
$$

Here, $\left\{\boldsymbol{u}_{k}^{n}\right\}$ and $\left\{v_{k}^{n}\right\}$ denote the sequences of $1 \times M$ random regressor vectors and zero-mean local measurement noises, respectively. Such linear models are wellsuited to approximate input-output relations for many practical applications [224]. The sequence $\left\{\boldsymbol{u}_{k}^{n}, v_{k}^{n}\right\}$ can generally be correlated as long as the remote past and distant future are asymptotically independent; see [101, Section II-C] for details and examples of such processes. For simplicity of presentation, we assume here that the sequence $\left\{\boldsymbol{u}_{k}^{n}, v_{k}^{n}\right\}$ is temporally white and spatially independent such that [182]:

$$
\begin{aligned}
& \mathbf{E}\left\{\left[\boldsymbol{u}_{k}^{n}\right]^{\prime} \boldsymbol{u}_{\underline{k}}^{l}\right\}=R_{d}^{n} \cdot \delta_{k \underline{k}} \cdot \delta_{n l} \\
& \mathbf{E}\left\{v_{k}^{n} v_{\underline{k}}^{l}\right\}=\sigma_{v, n}^{2} \cdot \delta_{k \underline{k}} \cdot \delta_{n l}
\end{aligned}
$$

where $\boldsymbol{x}^{\prime}$ denotes the transpose of vector $\boldsymbol{x}, R_{d}^{n}$ is positive-definite and $\delta_{i j}$ is the Kronecker delta function: $\delta_{i j}=1$ if $i=j$, and 0 otherwise. The noise sequence $\left\{v_{k}^{n}\right\}$ is further uncorrelated with the regression data $\left\{\boldsymbol{u}_{\underline{k}}^{l}\right\}$ for all $\{k, \underline{k}, n, l\}$ :

$$
\mathbf{E}\left\{\left[\boldsymbol{u}_{k}^{n}\right]^{\prime} v_{\underline{k}}^{l}\right\}=\mathbf{0} .
$$

The network of sensors then seeks to solve the least mean square parameter estimation problem

$$
\min _{\psi} \mathbf{E}\left\|\boldsymbol{Y}_{k}-\mathbf{u}_{k} \boldsymbol{\psi}\right\|^{2}
$$

where $\|\cdot\|$ denotes Euclidean norm and

$$
\mathbf{u}_{k} \stackrel{\text { defn }}{=} \operatorname{col}\left(\boldsymbol{u}_{k}^{1}, \ldots, \boldsymbol{u}_{k}^{N}\right), \quad \boldsymbol{Y}_{k} \stackrel{\text { defn }}{=} \operatorname{col}\left(y_{k}^{1}, \ldots, y_{k}^{N}\right)
$$




\subsubsection{Diffusion Least Mean Squares (LMS)}

We focus on the diffusion LMS algorithm [182] that adopts a peer-to-peer diffusion protocol and provides a distributed solution to the centralized parameter estimation problem (5.65). Sensors form social groups within which estimates are exchanged. Sensors then fuse the collected data and combine it with the local measurements to refine their estimates. Deploying such "social sensors" has been proved to improve the estimation performance, yet yield savings in computation, communication and energy expenditure [226, 225].

Recall the connectivity graph $G=(V, E)$, from Definition 5.4, and the closed neighborhood $N_{c}^{n}$ in (5.9). For simplicity of presentation, we assume the connectivity graph $G$ is fixed and strongly connected, i.e., there exists a path between each pair of nodes 23 . Let $0<\mu \ll 1$ be a small positive step size and define the weight matrix $W=\left[w_{i j}\right]$ for the diffusion protocol as follows:

$$
\begin{gathered}
W=I_{K}+\mu Q, \\
\left|q_{i j}\right| \leq 1, \quad \forall i, j, \quad q_{i j} \geq 0, \text { for } i \neq j, \\
Q \mathbf{1}=\mathbf{0}, \quad \text { and } Q^{\prime}=Q .
\end{gathered}
$$

The diffusion LMS then requires each sensor to employ a stochastic approximation algorithm of the form

$$
\begin{array}{cl}
\text { (Data Assimilation) } & \boldsymbol{\psi}_{k+1}^{n}=\boldsymbol{\phi}_{k}^{n}+\mu\left[\boldsymbol{u}_{k}^{n}\right]^{\prime}\left[y_{k}^{n}-\boldsymbol{u}_{k}^{n} \boldsymbol{\phi}_{k}^{n}\right] \\
\text { (Data Fusion) } & \boldsymbol{\phi}_{k+1}^{n}=\sum_{l \in N_{c}^{n}} w_{n l} \boldsymbol{\psi}_{k+1}^{l}
\end{array}
$$

where the second step is the local fusion of estimates via a linear combiner. Note that, since each sensor has a different neighborhood, the fusion rule (5.67) helps fuse data across the network into node's estimate. This enables the network to respond in real-time to the temporal and spatial variations in the statistical profile of the data.

Define the interpolated process associated with each sensor's estimate of the true parameter as follows:

$$
\boldsymbol{\psi}^{n, \mu}(t)=\boldsymbol{\psi}_{k}^{n} \quad \text { for } \quad t \in[k \mu,(k+1) \mu) .
$$

\footnotetext{
${ }^{23}$ Intermittent sensor failures can be captured by a random graph model where the probability that two sensors are connected is simply the probability of successful communication times the indicator function that shows the two sensors are neighbors in the underlying fixed graph. In this case, mean connectedness of the random graph is sufficient, i.e., $\lambda_{2}(\bar{L})>0$, where $\bar{L}=\mathbf{E} L_{n}, L_{n}$ denotes the Laplacian of the random graph process $\left\{G_{n}\right\}$, and $\lambda_{2}(\cdot)$ denotes the second largest eigenvalue; see [137] for details.
} 
Using the well-known ODE method for convergence analysis of stochastic approximation algorithms, it is shown in [101] that, as $\varepsilon \rightarrow 0$,

$$
\psi^{n, \mu}(t) \Rightarrow \boldsymbol{\psi}^{o} \quad \text { as } \quad t \rightarrow \infty
$$

To provide some intuition on the particular choice of the weight matrix in (5.66), consider the standard diffusion LMS algorithm in [182]: it uses the same updates as in (5.67), however, with weight matrix $\widehat{W}$ that is only assumed to be stochastic, i.e., $\widehat{W} \mathbf{1}=\mathbf{1}$. Define the network's global estimate of the true parameter as

$$
\boldsymbol{\Psi}_{k}=\operatorname{col}\left(\boldsymbol{\psi}_{k}^{1}, \ldots, \boldsymbol{\psi}_{k}^{N}\right)
$$

Let further

$$
\boldsymbol{U}_{k}=\operatorname{diag}\left(\boldsymbol{u}_{k}^{1}, \ldots, \boldsymbol{u}_{k}^{N}\right), \quad \widehat{\mathbf{W}}=\widehat{W} \otimes I_{M}
$$

where $\otimes$ represents the Kronecker product. The global recursion for the diffusion LMS across the network can then be expressed as

$$
\boldsymbol{\Psi}_{k+1}=\widehat{\mathbf{W}} \boldsymbol{\Psi}_{k}+\mu I_{N M} \boldsymbol{U}_{k}^{\prime}\left[\boldsymbol{Y}_{k}-\boldsymbol{U}_{k} \widehat{\mathbf{W}} \boldsymbol{\Psi}_{k}\right] .
$$

Remark 5.1. A classical stochastic approximation algorithm is of the form $\boldsymbol{\Psi}_{k+1}=$ $\boldsymbol{\Psi}_{k}+\mu g\left(\boldsymbol{\Psi}_{k}, \boldsymbol{v}_{k}\right)$, where $\boldsymbol{v}_{k}$ denotes a noise sequence and $0<\mu \ll 1$ denotes the step-size. Clearly, the global recursion for diffusion LMS (5.69) is not in such form because of the data fusion captured by the first term on the r.h.s in (5.69).

The following theorem shows that the diffusion LMS (5.69) is "equivalent" to a classical stochastic approximation algorithm. Below, we use the notation $\log (A)$ to denote the matrix logarithm of a matrix $A$. Equivalently, $B=\log (A)$ if $A=\exp (B)$ where $\exp (B)$ denotes the matrix exponential.

Theorem 5.6.1. Let $\widehat{W}$ be a stochastic matrix. Then, as the sampling time $\Delta \rightarrow 0$, the discrete-time data fusion recursion (5.69) is equivalent to a standard stochastic approximation algorithm of the form

$$
\begin{gathered}
\boldsymbol{\Psi}_{k+1}=\boldsymbol{\Psi}_{k}+\mu I_{N M}\left[\widetilde{\boldsymbol{W}} \boldsymbol{\Psi}_{k}+\boldsymbol{U}_{k}^{\prime}\left[\boldsymbol{Y}_{k}-\boldsymbol{U}_{k} \widehat{\boldsymbol{W}} \boldsymbol{\Psi}_{k}\right]\right], \\
\text { where } \widetilde{\boldsymbol{W}}=\ln (\widehat{\boldsymbol{W}}) / \Delta \text {, and } 0<\mu \ll 1
\end{gathered}
$$

in the sense that they both form discretizations of the same ODE

$$
\frac{d \Psi}{d t}=\widetilde{\boldsymbol{W}} \boldsymbol{\Psi}
$$


Proof. Suppose $\Delta$ time units elapse between two successive iterations of (5.69). Then, the diffusion protocol (the first term on the r.h.s of (5.69) ) can be conceived as a discretization of the ODE $d \Psi / d t=\widetilde{\boldsymbol{W}} \boldsymbol{\Psi}$, where $\exp (\widetilde{\boldsymbol{W}} \Delta)=\widehat{\boldsymbol{W}}$. Taking Taylor expansion yields

$$
\widehat{\boldsymbol{W}}=I+\Delta \widetilde{\boldsymbol{W}}+o(\Delta), \quad \text { where } o(\Delta) \rightarrow 0 \text { as } \Delta \rightarrow 0
$$

Therefore, as $\Delta \rightarrow 0$, the standard diffusion LMS (5.69) is equivalent to the standard stochastic approximation iterate (5.70).

Further inspection of the matrix $\widetilde{W}$ in (5.70) shows that, since $\widetilde{\boldsymbol{W}}=$ $\lim _{\Delta \rightarrow 0}(\exp (\widetilde{\boldsymbol{W}} \Delta)-I) / \Delta$,

$$
\widetilde{W} \mathbf{1}=\mathbf{0}, \text { and } \widetilde{w}_{i j}>0 \text { for } i \neq j .
$$

Now, comparing (5.72) with (5.66), together with Theorem 5.6.1, confirms the equivalence between the standard diffusion protocol [182] and the diffusion protocol constructed by using the weight matrix (5.66). The advantages of the particular choice of the weight matrix (5.66) in the adaptive filtering algorithm (5.67) are however threefold: (i) both data assimilation and fusion takes place on the same timescale; (ii) simpler derivation of the known results can be obtained by employing the powerful ordinary differential equation (ODE) method [29, 167]; (iii) one can use weak convergence methods [167, Chapter 8] to show how responsive the adaptive filtering algorithm is to the time-variations of the true parameter.

\subsubsection{Energy-Aware Activation Control of Diffusion LMS}

Can one make the above decentralized solution to the sensing problem even more efficient by allowing sensors to activate only when their contributions outweigh the activation costs? We equip the sensors with an activation mechanism that, taking into account the spatial-temporal correlation of their measurements, prescribes sensors to sleep when the the energy cost of acquiring new measurement outweighs its contribution to the estimation task. When a node is active, it updates its estimate and performs fusion of its own estimate with those received from neighboring nodes, whereas inactive nodes do not update their estimates. More precisely, each sensor runs local updates of the form

$$
\boldsymbol{\psi}_{k+1}^{n}=\left\{\begin{array}{lll}
\boldsymbol{\phi}_{k}^{n}+\mu\left[\boldsymbol{u}_{k}^{n}\right]^{\prime}\left[y_{k}^{n}-\boldsymbol{u}_{k}^{n} \boldsymbol{\phi}_{k}^{n}\right], & \text { if } \mathrm{a}_{k}^{n}=1 \text { (Active) } \\
\boldsymbol{\psi}_{k}^{n}, & \text { if } \mathrm{a}_{k}^{n}=0 \text { (Sleep) }
\end{array}\right.
$$


where $\phi_{k}^{n}$ is the fused local estimate, defined in (5.67). Due to the interdependence of sensors' activation behavior, a game-theoretic approach is a natural choice to model their interaction.

The problem of each sensor $n$ is then to successively pick action $\mathrm{a}_{k}^{n}$ from the set $\mathcal{A}^{n}=\{0$ (sleep), 1 (activate) $\}$ to strategically optimize a utility function. This utility function captures the trade-off between energy expenditure and the "value" of sensor's contribution. Let $\boldsymbol{\Psi}=\left[\boldsymbol{\psi}^{1}, \cdots, \boldsymbol{\psi}^{N}\right]$ denote the profile of estimates across the network. The utility function for each sensor $n$ can then be defined as follows:

$$
U^{n}\left(\mathrm{a}^{n}, \mathbf{a}^{-n} ; \boldsymbol{\Psi}\right)=U_{L}^{n}\left(\mathrm{a}^{n}, \mathbf{a}^{N^{n}} ; \boldsymbol{\Psi}\right)+U_{G}^{n}\left(\mathrm{a}^{n}, \mathbf{a}^{S^{n}}\right) .
$$

The local utility function $U_{L}^{n}(\cdot)$ captures the trade-off between the value of the measurements collected by sensor $n$ and the energy costs associated with it. If too many of sensor $n$ 's neighbors activate simultaneously, excessive energy is consumed due to the spatial-temporal correlation of sensor measurements - that is, the data collected by sensor $n$ is less valuable. On the other hand, if too few of sensor $n$ 's neighbors activate, their fused estimates lack "innovation." The local utility of sensor $k$ is then given by

$$
\begin{aligned}
U_{L}^{n}\left(\mathrm{a}^{n}, \mathbf{a}^{N^{n}} ; \boldsymbol{\Psi}\right)= & {\left[K_{l, 1}\left(1-\exp \left(-\gamma_{l}\left\|\boldsymbol{\psi}^{n}-\boldsymbol{\phi}^{n}\right\|^{2} s\left(\eta^{k}\right)\right)\right)\right.} \\
& \left.-K_{l, 2}\left(E_{\mathrm{Tx}}\left(\eta^{n}\right)+E_{\mathrm{Act}}\right)\right] I\left\{\mathrm{a}^{n}=1\right\}
\end{aligned}
$$

where

$$
\eta^{k}\left(\mathrm{a}^{n}, \mathbf{a}^{N^{n}}\right)=\mathrm{a}^{n}+\sum_{l \in N^{n}} \mathrm{a}^{l} .
$$

In addition, $K_{l, 1}$ and $\gamma_{l}$ are the pricing parameters related to the "reward" associated with the data collected by sensor $n, K_{l, 2}$ is the pricing parameter related to the energy costs associated with activation $E_{\text {Act }}$ and broadcasting measurements $E_{\mathrm{Tx}}(\cdot)$. Finally, $s\left(\eta^{n}\right)$ denotes the probability of successful transmission24. A

${ }^{24}$ The probability of successful transmission is given by

$$
s\left(\eta^{n}\right)=\sum_{m=0}^{B_{\max }} p\left(\eta^{n}\right)\left(1-p\left(\eta^{n}\right)\right)^{m}
$$

Here, $B_{\max }$ denotes the maximum number of back-offs, $p\left(\eta^{n}\right)=\left(1-q^{n}\right)^{\eta^{n}-1}$ denotes the probability that the channel is clear, and $q^{n}$ represents the probability that sensor $n$ is transmitting at a given time; see [161, p. 6099] for details in the unslotted CSMA/CA scheme. 
sensor is thus motivated to activate when majority of its neighbors are in the sleep mode and/or its estimate $\psi^{n}$ is far from the local fused estimate $\phi^{n}$.

The global utility function $U_{G}^{n}(\cdot)$ concerns the connectivity of the network and diffusion of estimates. Let $\mathcal{R}_{r}^{n}$ denote the set of sensors within radius $r$ from sensor $n$ excluding the neighborhood $N_{c}^{n}$. Define the number of active sensors in $\mathcal{R}_{r}^{n}$ by

$$
\zeta^{n}\left(\mathrm{a}^{n}, \mathbf{a}^{S^{n}}\right)=\mathrm{a}^{n}+\sum_{l \in \mathcal{R}_{r}^{n}} a^{l}
$$

The global utility of sensor $n$ is then given by

$$
U_{G}^{n}\left(\mathrm{a}^{n}, \mathbf{a}^{S^{n}}\right)=K_{g}\left(e^{-\gamma_{g} \zeta^{n}}\right) I\left\{\mathrm{a}^{n}=1\right\}
$$

where $K_{g}$ and $\gamma_{g}$ are the pricing parameters. Higher $\zeta^{n}$ lowers the global utility due to less importance of sensor $n$ 's contribution to the diffusion of estimates across the network and keeping connectivity in $\mathcal{R}_{r}^{k}$. Sensor $n$ is thus motivated to activate when majority of the nodes in its geographic region $\mathcal{R}_{r}^{n}$ are in the sleep mode.

Each sensor $n$ realizes $\eta_{k}^{n}=\eta^{n}\left(\mathrm{a}_{k}^{n}, \mathbf{a}_{k}^{N^{n}}\right)$ as a consequence of receiving estimates $\left\{\boldsymbol{\psi}_{k}^{l}\right\}_{l \in N^{n}}$ from neighbors, therefore, is able to evaluate its local utility at each period $k$. However, it does not observe the actions of non-neighbors, therefore, does not realize $\zeta_{k}^{n}=\zeta^{n}\left(\mathrm{a}_{k}^{n}, \mathbf{a}_{k}^{S^{n}}\right)$ and is unable to evaluate its global utility. The above game-theoretic model for activation control of sensors matches the setting of \$5.3.2. Therefore, we employ Algorithm 6 with the above utility function to devise the activation mechanism. This mechanism is then embedded into the diffusion LMS algorithm such that the overall energy-aware diffusion LMS forms a two-timescale stochastic approximation algorithm: the fast timescale corresponds to the game-theoretic activation mechanism (step-size $\varepsilon$ ) and the slow timescale is the diffusion LMS (step-size $\mu=\mathcal{O}\left(\varepsilon^{2}\right)$ ).

The resulting energy-aware diffusion LMS algorithm is summarized in Algorithm 8 , 


\begin{tabular}{l}
$\overline{\text { Algorithm 8 Energy-Aware Diffusion LMS }}$ \\
\hline Initialization: Set $0<\delta<1, \mu^{n}>A^{n}\left|U_{\max }^{n}-U_{\min }^{n}\right|, \mathbf{p}_{0}^{n}=\left[\frac{1}{2}, \frac{1}{2}\right]^{\prime}$, and \\
$R_{0}^{L, n}=R_{0}^{G, n}=\mathbf{0}_{2 \times 2}$. Choose $0<\varepsilon, \rho \ll 1$ such that $\mu=\mathcal{O}\left(\varepsilon^{2}\right)$, and \\
$\boldsymbol{\psi}_{0}^{n}=\boldsymbol{\phi}_{0}^{n}=\mathbf{0}$.
\end{tabular}

Step 1: Node Activation. Choose $\mathrm{a}_{k}^{n} \in\{1$ (Activate), 0 (Sleep) $\}$ according to the randomized strategy $\mathbf{p}_{k}^{n}$ :

$$
p_{k}^{n}(i)= \begin{cases}(1-\delta) \min \left\{\frac{1}{\mu^{n}}\left|r_{k}^{n}\left(\mathrm{a}_{k-1}^{n}, i\right)\right|^{+}, \frac{1}{2}\right\}+\frac{\delta}{2}, & \mathrm{a}_{k-1}^{n} \neq i \\ 1-\sum_{j \neq i} p_{k}^{n}(j), & \mathrm{a}_{k-1}^{n}=i\end{cases}
$$

Step 2: Diffusion LMS.

$$
\boldsymbol{\psi}_{k+1}^{n}= \begin{cases}\boldsymbol{\phi}_{k}^{n}+\mu\left[\boldsymbol{u}_{k}^{n}\right]^{\prime}\left[y_{k}^{n}-\boldsymbol{u}_{k}^{n} \boldsymbol{\phi}_{k}^{n}\right], & \text { if } \mathrm{a}_{k}^{n}=1 \text { (Active), } \\ \boldsymbol{\psi}_{k}^{n}, & \text { if } \mathrm{a}_{k}^{n}=0 \text { (Sleep) }\end{cases}
$$

Step 3: Estimate Exchange. If $\mathrm{a}_{k}^{n}=1$ : (i) Transmit $\psi_{k}^{n}$ to neighbors $N^{n}$ and collect $\left\{\boldsymbol{\psi}_{k}^{l}\right\}_{l \in N^{n}}$; (ii) set

$$
\mathrm{a}_{k}^{l}= \begin{cases}1, & \text { if node } n \text { receieves node } l \text { 's estimate } \psi_{k}^{l}, \\ 0, & \text { otherwise, }\end{cases}
$$

and

$$
\widehat{\boldsymbol{\psi}}_{k}^{l}= \begin{cases}\text { received estimate, }, & \text { if } \mathrm{a}_{k}^{l}=1, \\ \boldsymbol{\psi}_{k-1}^{l}, & \text { if } \mathrm{a}_{k}^{l}=0 .\end{cases}
$$

If $\mathrm{a}_{k}^{n}=0$, go to Step 5 .

Step 4: Fusion of Local Estimates.

$$
\boldsymbol{\psi}_{k}^{n}=\sum_{l \in N_{c}^{n}} w_{n l} \widehat{\boldsymbol{\psi}}_{k}^{l} .
$$

Step 5: Regret Update. Run 'Step 3' in Algorithm6.

Step 6: Recursion. Set $k \leftarrow k+1$, and go Step 1 .

In such two timescale algorithms, the fast time-scale will see the slow component as quasi-static while the slow component sees the fast one near equilibrium. By virtue of the results of \$5.4, consistency of the diffusion LMS (5.68), and treatment of asynchronous stochastic approximation algorithms, it is shown in [101] 


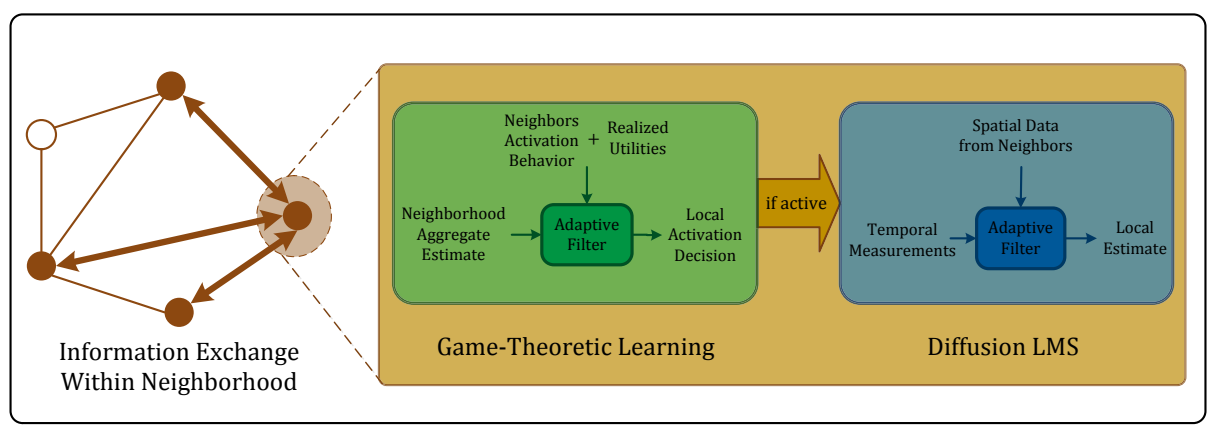

(a) Local Adaptation and Learning

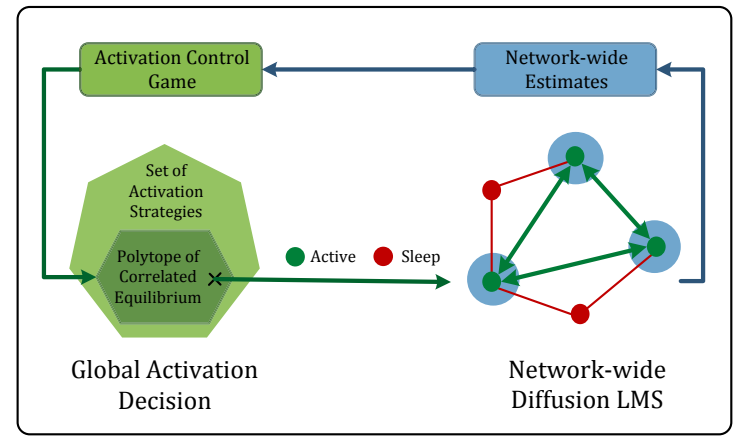

(b) Global Behavior

Figure 5.6: Energy-aware diffusion LMS is asymptotically consistent, yet the global activation behavior along the way tracks the correlated equilibria set of the activation control game.

that, if each sensor individually follows Algorithm 6 , the following results hold:

(i) As $\mu \rightarrow 0, \boldsymbol{\psi}^{n, \mu}(t)$ converges weakly to $\boldsymbol{\psi}^{o}$ across the network. That is, the energy-aware diffusion LMS is consistent.

(ii) As $\varepsilon \rightarrow 0, \boldsymbol{z}^{\varepsilon}(\cdot)$ converges weakly to $\mathcal{C}_{\epsilon}(\boldsymbol{\Psi}(\cdot))$. That is, the global activation behavior across the network belongs to the correlated $\epsilon$-equilibria set of the underlying activation control game.

The local and global behavior of the energy-aware diffusion LMS in illustrated in Figure 5.6. Note that, since the utility function (5.74) is a function of the estimates 

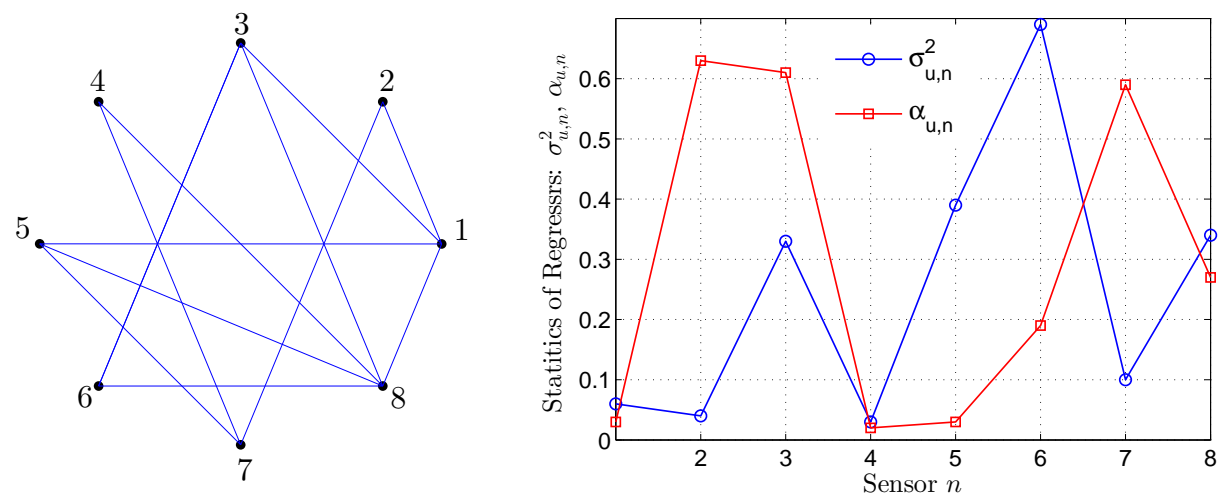

Figure 5.7: Network topology and sensors' regressors statistics.

across the network, so is the correlated equilibria set of the underlying game-it slowly evolves as the parameter estimates across the network $\boldsymbol{\Psi}_{k}$ change over time.

\subsubsection{Numerical Study}

Fig. 5.7 depicts the network topology that we study in this example. It further illustrates the statistics of each sensor's sequence of regressors that is generated by a Gaussian Markov source with local correlation function of the form $r(\tau)=$ $\sigma_{\boldsymbol{u}, n}^{2} \alpha_{\boldsymbol{u}, n}^{|\tau|}$, where $\alpha_{\boldsymbol{u}, n}$ is the correlation index. We define $Q=\left[q_{k l}\right]$ in (5.66) as

$$
q_{n l}= \begin{cases}\frac{1}{\left|N_{c}^{n}\right|}, & l \in N_{c}^{n}, \\ 0, & \text { otherwise. }\end{cases}
$$

It is further assumed that sensors are equipped with Chipcon CC2420 transceiver chipset which implements CSMA/CA protocol for exchanging estimates with neighbors25. For brevity of presentation, we skip the detailed model description and expressions for $E_{\mathrm{Tx}}(\cdot)$ and $E_{\mathrm{Act}}$ in (5.75); the interested reader is referred

\footnotetext{
${ }^{25}$ The ZigBee/IEEE 802.15.4 standard is currently a leading choice for low-power communication in wireless sensor networks. It employs a CSMA/CA scheme for multiple access data transmission. In networks with tight energy constraints, the non-beacon-enabled (unslotted CSMA/CA) mode is more preferable as the node receivers do not need to switch on periodically to synchronize to the beacon.
} 
to [101], [161, Appendix] for the details. We further assume the noise $v_{n}^{k}$ is i.i.d. with $\sigma_{v, k}^{2}=10^{-3}$, and $\mu=0.01$.

Define the network excess mean square error:

$$
\mathrm{EMSE}^{\mathrm{net}}=\frac{1}{N} \sum_{n=1}^{N} \mathrm{EMSE}^{n}
$$

where $\mathrm{EMSE}^{n}$ denotes the EMSE for sensor $n$. $\mathrm{EMSE}^{\text {net }}$ is simply obtained by averaging $\mathbf{E}\left|\boldsymbol{u}_{k}^{n}\left(\boldsymbol{\psi}^{o}-\boldsymbol{\phi}_{k-1}^{k}\right)\right|^{2}$ across all sensors in the network. Figure 5.8 demonstrates the trade-off between energy expenditure in the network and the rate of convergence of the diffusion LMS algorithm in terms of EMSE ${ }^{\text {net }}$ after $10^{4}$ iterations. Sensors become more conservative by increasing the energy consumption parameter $K_{l, 2}$ and, accordingly, activate less frequently due to receiving lower utilities; see (5.75). This reduces the average proportion of active sensors and increases $\mathrm{EMSE}^{\text {net }}$ due to recording fewer measurements and less frequent fusion of neighboring estimates. Increasing the pricing parameters $\gamma_{l}$, in (5.75), and $\gamma_{g}$, in (5.76), has the same effect as can be observed in Figure 5.8. The global performance of Algorithm 8 is further compared with the standard diffusion LMS [182] in Figure 5.9. As the pricing parameters $K_{l, 1}$ in (5.75) (corresponding to the contribution of sensors in local parameter estimation) and $K_{g}$ in (5.76) (corresponding to the contribution of sensors in local diffusion) increase, sensors activate more frequently. As shown in Figure 5.9, this improves the rate of convergence of the energy-aware diffusion LMS algorithm.

\subsection{Closing Remarks}

In this chapter, we used non-cooperative game-theoretic learning as a model for interactive sensing with social sensors. Besides the competitive situations, equally abundant are interactions wherein multiple agents cooperate to reach a common goal. For instance, family members and friends register to the same long-distance service to take advantage of more affordable rates. Similarly, multiple airlines may form alliances to provide convenient connections between as many airports as possible so as to increase profit [234]. Associated with each formed group of cooperating parties, namely, coalitions, there corresponds a productive value which captures how fruitful their collaboration is in fulfilling the coalition's goal. Such a value may depend not only on parties that form a coalition and their connection (influence), but also on those who are excluded from the coalition. For 


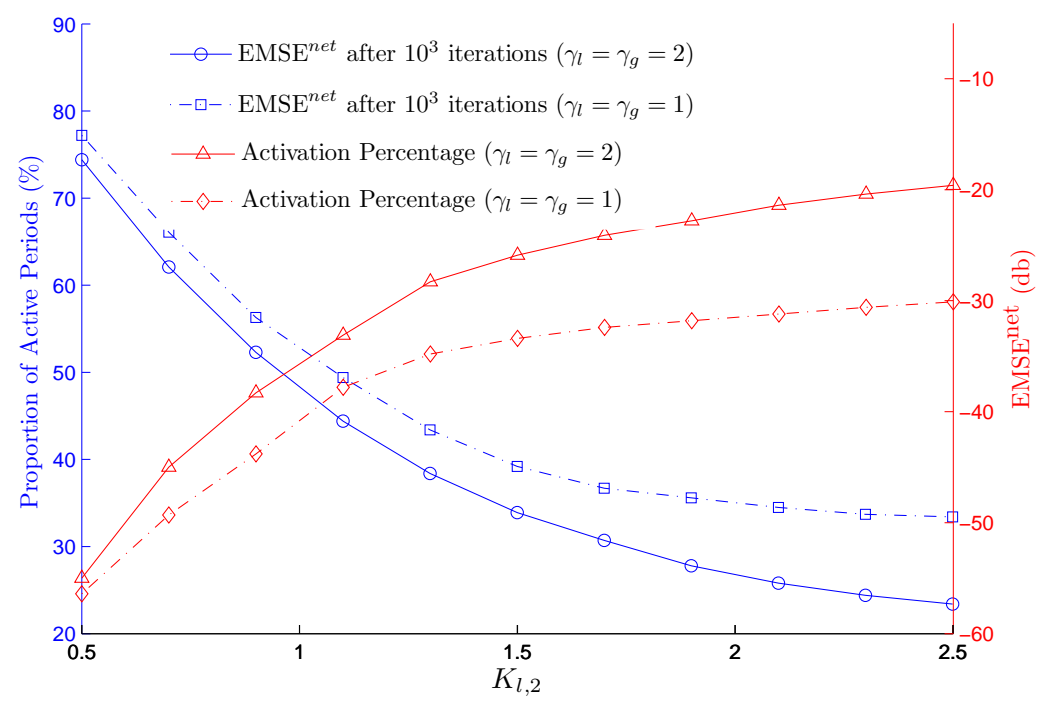

Figure 5.8: Energy expenditure vs. estimation accuracy after $10^{4}$ iterations.

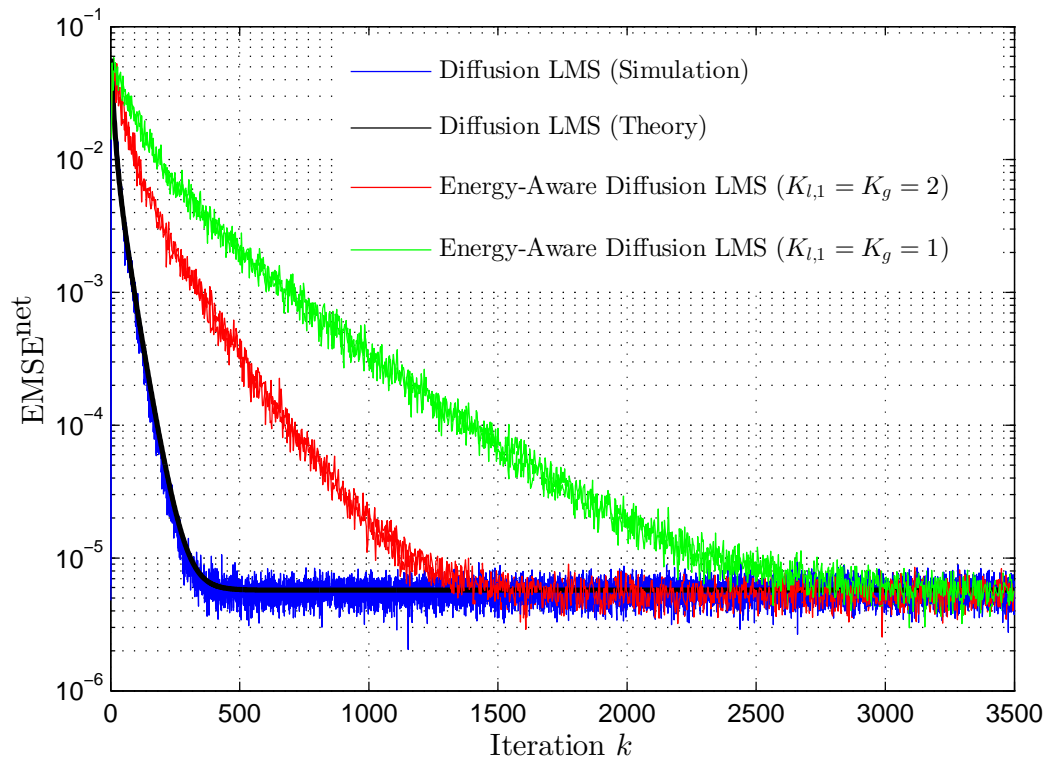

Figure 5.9: Network excess mean square error EMSE ${ }^{\text {net }}$. 
instance, registering to a particular long-distance service clearly has advantages to the members of a family; however, due to the associated overhead costs, it may limit communication with friends who are registered to a different service. Therefore, any competition (if existent at all) will be among coalitions, rather than parties.

In such situations the main issue is to obtain a fair distribution of the joint revenues from cooperation so as to form stable coalitions. For instance, the productive value may be split based on the relative contributions or relations (influence) of different parties within each coalition. In the presence of multiple coalitions, each agent naturally favours the one that ensures the highest payoff. Allocation of shares from the joint revenues, thus, impacts the structure of formed coalitions and their stability.

Formation of such stable coalitions can be naturally modelled and analysed in a particular strain of the cooperative game framework, namely, coalition formation games. Coalition formation is of particular significance in several economic, political and social contexts such as cartel formation, lobbies, customs unions, conflict, public goods provision, political party formation, etc. [216, 217]. The single-valued Shapley value [230, 122], named in honour of Lloyd Shapley, and the set-valued core 26 are the most prominent solution concepts in the context of cooperative games.

An extension of the game-theoretic learning approach to interactive sensing is to model formation of stable coalitions of sensors that collaborate to gather data about the same phenomenon of interest; cf. [98, 99] for two instances of cooperative game-theoretic learning approach to target localization and resource allocation problems.

Another direction for further research is to explore the adaptive learning algorithms of game-theoretic nature in large-scale discrete optimization problems, where the optimal solution is obtained by collecting observations from many separate sources via a social network [96, 103]. One such problem is choosing influential sets of individuals to maximize the spread of influence through a social network, which was first formulated as a discrete optimization in the seminal work [142]: "if we can try to convince a subset of individuals to adopt a new product or innovation, and the goal is to trigger a large cascade of further adoptions, which set of individuals should we target?" For more recent works in this area, the reader is referred to [57, 58].

\footnotetext{
${ }^{26}$ The formal concept of the core for cooperative games in characteristic form was first introduced by Gillies [104] and Shapley [229]; see [194, Ch. 6] for additional background.
} 


\section{Chapter 6}

\section{Summary}

The motivation for this monograph stems from understanding how individuals interact in a social network and how simple local behavior can result in complex global behavior. The underlying tools employed in this monograph are widely used by the electrical engineering research community in the areas of signal processing, control, information theory and network communications. In what follows, we summarize the main results discussed in detail in this monograph:

Chapter 2 considered social learning models for interaction among sensors where agents use their private observations along with actions of other agents to estimate an underlying state of nature. We considered extensions of the basic social learning paradigm to online reputation systems in which agents communicate over a social network. Despite the apparent simplicity in these information flows, the systems exhibit unusual behavior such as herding and data incest. Also, an example of social-learning for change detection was considered.

Chapter 3 analyzed the dynamics of a duplication-deletion graph where at each time instant, one node can either join or leave the graph (an extension to the duplication model of [62, 211]). The power law component for such graph was computed. Also, a Markov-modulated random graph was proposed to model the social networks whose dynamics evolve over time according to a finite-state Markov chain. Using the stochastic approximation algorithms, the probability mass function of degree of each node (degree distribution) was estimated. Then, an upper bound was derived for the distance between the estimated and the expected degree distribution. As a result of this bound, we showed that the scaled tracking error between the expected degree distribution and the estimated one weakly converges to a diffusion process. From that, the covariance of this error can be computed. Finally, we presented a discussion on simulating graphical realizations of a de- 
gree sequence which is a problem of much interest in numerical studies of social networks.

Chapter 4 discussed the Susceptible-Infected-Susceptible (SIS) model for diffusion of information in social networks. By using the mean field dynamics, the degree distribution was shown to satisfy an ordinary differential equation. As a result, the probability of a link pointing to an infected node satisfies a scalar differential equation. Given these dynamics for the state of information diffusion, sampling of the nodes in the network can be viewed as a noisy measurements of the state. We outlined two types of sampling individuals in a network that are of recent interest-namely, social sampling and respondent-driven sampling. Given these noisy measurements, estimation of the underlying state was formulated as a Bayesian filtering problem.

Chapter 5] discussed, at length, a non-Bayesian formulation, where agents seek to achieve coordination in decision making by optimizing their own utility functions. We adopted a non-cooperative game-theoretic formulation and considered three protocols for information exchange among interacting agents. The well-known game-theoretic learning algorithm — namely, the Hart \& Mas-Colell's "regret-matching" procedure-was presented and extended to accommodate the information flow protocols that are typical in interactions among agents in social networks. Interestingly enough, we have shown that, while each individual has limitations in sensing the environment and communicating with other agents, the coordinated behavior among agents can lead to the manifestation of sophisticated behavior at the network level. More precisely, the global behavior of agents turn out to be distributed according to randomized strategies drawn from the convex polytope of correlated equilibria. Instances of such emergent global behavior are even observed in nature, e.g., fish schooling and birds flight in formation [49, 246].

In this monograph, to give a flavor of the various averaging analysis methods, we used a variety of analysis tool to analyse the asymptotic properties of the presented stochastic approximation algorithms: (a) Mean square convergence: Chapter 3 used this method to derive bounds on the difference between the estimated and the expected degree distribution of a Markov-modulated random graph; (b) Maximum deviation bounds: Chapter 4 employed this method to derive an exponential bound (in terms of the number of agents) for the probability of deviation of the sample path of the infected distribution from the mean field dynamics in finite time interval; (c) Weak convergence: Chapter 3 and Chapter 5 use weak convergence methods to analyze the convergence of stochastic approximation algorithms. In Chapter 3 these methods were used to specify how the empirical distribution of a Markov chain can be tracked and how the tracking er- 
rors satisfy a switched Markovian diffusion process. Finally Chapter 5 used weak convergence methods to derive the limit system representing the asymptotics of the game-theoretic decision making procedure. This method was shown to be strong enough to tackle Markovian evolution of the parameters underlying the non-cooperative game model.

There exist other important problems in the context of social networks that have been extensively studied in the literature, hence, were not included in this monograph. Examples include consensus formation [132, Chapter 8], [149, 240], metrics for measuring networks (other than degree distribution) [132, Chapter 2], [253], small world [144, 254, 255], cooperative models of network formation [131, Chapter 1], [132, Chapter 12], [233], behavior dynamics in peer-to-peer mediasharing social networks [112, 267], and privacy and security modeling [169, 179], to name a few. The interested reader can find detailed discussions on these topics in the above references and the references therein. 


\section{Appendix A}

\section{Appendix to Chapter 3}

\section{A.1 Proof of Theorem 3.3.1}

The proof is based on the proof of Lemma 4.1 in [61, Chapter 4, p79]. To compute the expected degree distribution of the Markov-modulated random graph, we obtain a relation between the number of nodes with specific degree at time $n$ and the degree distribution of the graph at time $n-1$. For the sake of notational convenience, we drop the dependency of probabilities of connection and deletion on $\theta$ and we denote $p(\theta)$ and $q(\theta)$ by $p$ and $q$. Given the resulting graph at time $n$, the aim is to find the expected number of nodes with degree $i+1$ at time $n+1$. The following events can occur that result in a node with degree $i+1$ at time $n+1$ :

- Degree of a node with degree $i$ increments by one in the duplication step (Step 2 of Algorithm 2) and remains unchanged in the deletion step (Step 3):

- A node with degree $i$ is chosen at the duplication step as a parent node and remains unchanged in the deletion step. The probability of occurrence of such an event is

$$
\left(1-\frac{q(i+1)+q(1+p i)-q(1+p i)(i+1) / N_{n}}{N_{n}}\right) \frac{f_{n}(i)}{N_{n}}
$$

the probability of choosing a node with degree $i$ is $\frac{f_{n}(i)}{N_{n}}$ and the probability of the event that this node remains unchanged in the deletion step is 1

$$
1-\frac{q(i+1)+q(1+p i)-q(1+p i)(i+1) / N_{n}}{N_{n}} .
$$

\footnotetext{
${ }^{1}$ The deletion step (Step 3 of Algorithm 2) comprises an edge-deletion step and a duplication step. The probability that the degree of node with degree $i$ changes in the edge-deletion step is
} 
- One neighbor of a node with degree $i$ is selected as a parent node; the parent node connects to its neighbors (including the node with degree $i$ ) with probability $p$ in the edge-duplication part of Step 2. The probability of such an event is

$$
\frac{f_{n}(i) p i}{N_{n}}\left(1-\frac{q(i+2)+q(1+p(i+1))-q(1+p(i+1))(i+2) / N_{n}}{N_{n}}\right) .
$$

Note that the node whose degree is incremented by one in this event should remain unaffected in Step 3; the probability of being unchanged in Step 3 for such a node is

$$
1-\frac{q(i+2)+q(1+p(i+1))-q(1+p(i+1))(i+2) / N_{n}}{N_{n}} .
$$

- A node with degree $i+1$ remains unchanged in both Step 2 and Step 3 of Algorithm 2:

- Using the same argument as above, the probability of such an event is

$$
f_{n}(i+1)\left(1-q \frac{i+3+p(i+1)-\frac{(1+p(i+1))(i+2)}{N_{n}}}{N_{n}}\right)\left(1-\frac{p(i+1)+1}{N_{n}}\right) .
$$

- A new node with degree $i+1$ is generated in Step 2:

- The degree of the most recently generated node (in the vertex- duplication part of Step 2) increments to $i+1$; the new node connects to " $i$ " neighbors of the parent node and remains unchanged in Step 3. The probability of this scenario is

$\frac{q(i+1)}{N_{n}}$; either this node or one of its neighbors should be selected in the edge-deletion step. Also given that the degree of this node dose not change in the edge-deletion step, if either this node or one of its neighbor is selected in the duplication step (within Step 3) then the degree of this node increments by one with probability $\frac{1+p i}{N_{n}}$. Therefore, the probability that the degree of a node of degree $i$ remains unchanged in Step 3 is

$$
1-\frac{q(i+1)+q(1+p i)-q(1+p i)(i+1) / N_{n}}{N_{n}} .
$$

Note that for simplicity in our analysis, we assumed that the nodes whose degrees are changed in the edge-deletion part of Step 3, remain unchanged in the duplication part of Step 3 at that time instant. Also, the new node, which is generated in the vertex-duplication step of Step 2, remains unchanged in Step 3. Therefore, although the degree of parent node becomes $i+1$ in this case but this node is treated as a node with degree $i$ in Step 3 of Algorithm 2 


$$
\left(1-q \frac{i+3+p(i+1)-\frac{(1+p(i+1))(i+2)}{N_{n}}}{N_{n}}\right) \sum_{j \geq i} \frac{f_{n}(j)}{N_{n}}\left(\begin{array}{c}
j \\
i
\end{array}\right) p^{i}(1-p)^{j-i}
$$

- Degree of a node with degree $i+2$ decrements by one in Step 3:

- A node with degree $i+2$ remains unchanged in the duplication step and one of its neighbors is eliminated in the deletion step. The probability of this event is

$$
q\left(\frac{i+2}{N_{n}}\right)\left(1-\frac{p(i+2)+1}{N_{n}}\right)
$$

- A node with degree $i+1$ is generated in Step 3:

- The degree of the node generated in the vertex-duplication part of duplication step within Step 3 increments to $i+1$. The probability of this event is

$$
q \sum_{j \geq i} \frac{1}{N_{n}} f_{n}(j)\left(\begin{array}{l}
j \\
i
\end{array}\right) p^{i}(1-p)^{j-i}
$$

- Degree of a node with degree $i$ increments by one in Step 3:

- A node with degree $i$ remains unchanged in Step 2 and its degree increments by one in the duplication part of Step 3. The corresponding probability is

$$
\frac{q(1+p i)}{N_{n}}\left(1-\frac{1+p i}{N_{n}}\right)
$$

Let $\Omega$ denote the set of all arbitrary graphs and $\mathcal{F}_{n}$ denote the sigma algebra generated by graphs $G_{\tau}, \tau \leq n$. Considering the above events that result in a node with degree $i+1$ at time $n+1$, the following recurrence formula can be derived for the conditional expectation of $f_{n+1}(i+1)$ : 


$$
\begin{aligned}
& \mathbf{E}\left\{f_{n+1}(i+1) \mid \mathcal{F}_{n}\right\}= \\
& \left(1-q \frac{i+3+p(i+1)-\frac{(1+p(i+1))(i+2)}{N_{n}}}{N_{n}}\right)\left(1-\frac{p(i+1)+1}{N_{n}}\right) f_{n}(i+1) \\
& +\left(1-\frac{q(i+1)+q(1+p i)-q(1+p i)(i+1) / N_{n}}{N_{n}}\right)\left(\frac{1+p i}{N_{n}}\right) f_{n}(i) \\
& +\left(1-q \frac{i+3+p(i+1)-\frac{(1+p(i+1))(i+2)}{N_{n}}}{N_{n}}\right) \sum_{j \geq i} \frac{f_{n}(j)}{N_{n}}\left(\begin{array}{l}
j \\
i
\end{array}\right) p^{i}(1-p)^{j-i} \\
& +q \sum_{j \geq i} \frac{f_{n}(j)}{N_{n}}\left(\begin{array}{l}
j \\
i
\end{array}\right) p^{i}(1-p)^{j-i}+q\left(\frac{i+2}{N_{n}}\right)\left(1-\frac{p(i+2)+1}{N_{n}}\right) f_{n}(i+2) \\
& +\frac{q(1+p i)}{N_{n}}\left(1-\frac{1+p i}{N_{n}}\right) f_{n}(i) \text {. }
\end{aligned}
$$

Let $\bar{f}_{n}^{\theta}(i)=\mathbf{E}\left\{f_{n}(i) \mid \theta_{n}=\theta\right\}$. By taking expectation of both sides of A.1 with respect to trivial sigma algebra $\{\Omega, \emptyset\}$, the smoothing property of conditional expectations yields:

$$
\begin{aligned}
& \bar{f}_{n+1}^{\theta}(i+1)= \\
& \quad\left(1-q \frac{\left.i+3+p(i+1)-\frac{(1+p(i+1))(i+2)}{N_{n}}\right)}{N_{n}}\right)\left(1-\frac{p(i+1)+1}{N_{n}}\right) \bar{f}_{n}^{\theta}(i+1) \\
& +\left(1-\frac{q(i+1)+q(1+p i)-\frac{q(1+p i)(i+1)}{N_{n}}}{N_{n}}\right)\left(\frac{1+p i}{N_{n}}\right) \bar{f}_{n}^{\theta}(i) \\
& +\left(1-q \frac{i+3+p(i+1)-\frac{(1+p(i+1))(i+2)}{N_{n}}}{N_{n}}\right) \sum_{j \geq i} \frac{\bar{f}_{n}^{\theta}(j)}{N_{n}}\left(\begin{array}{c}
j \\
i
\end{array}\right) p^{i}(1-p)^{j-i} \\
& +q \sum_{j \geq i} \frac{1}{N_{n}} \bar{f}_{n}^{\theta}(j)\left(\begin{array}{c}
j \\
i
\end{array}\right) p^{i}(1-p)^{j-i}+q\left(\frac{i+2}{N_{n}}\right)\left(1-\frac{p(i+2)+1}{N_{n}}\right) \bar{f}_{n}^{\theta}(i+2) \\
& +\frac{q(1+p i)}{N_{n}}\left(1-\frac{1+p i}{N_{n}}\right) \bar{f}_{n}^{\theta}(i) .
\end{aligned}
$$

Assuming that size of the graph is sufficiently large, each term like $\frac{\bar{f}_{n}\left(i^{\prime}\right)}{N_{n}^{2}}$ can be neglected. Also, taking functional dependencies of $p$ and $q$ on $\theta$ into account, Eq. 
(A.2) can be written as

$$
\begin{aligned}
\bar{f}_{n+1}^{\theta}(i+1) & =\left(1-\frac{q(\theta)(i+2)+q(\theta)(p(\theta)(i+1)+1)}{N_{n}}\right) \bar{f}_{n}^{\theta}(i+1) \\
& +\left(\frac{(1+p(\theta) i) q(\theta)}{N_{n}}\right) \bar{f}_{n}^{\theta}(i)+q(\theta)\left(\frac{i+2}{N_{n}}\right) \bar{f}_{n}^{\theta}(i+2) \\
& +q(\theta) \sum_{j \geq i} \frac{1}{N_{n}} \bar{f}_{n}^{\theta}(\theta, j)\left(\begin{array}{c}
j \\
i
\end{array}\right) p\left(\theta_{n+1}\right)^{i}\left(1-p\left(\theta_{n+1}\right)\right)^{j-i} .
\end{aligned}
$$

Using (A.2), we can write the following recursion for the $(i+1)$-th element of $\bar{g}^{\theta}(n+1)$ :

$$
\begin{aligned}
\bar{g}_{n+1}^{\theta}(i+1) & =\left(\frac{N_{n}-(q(\theta)(i+2)+q(\theta)(p(\theta)(i+1)+1))}{N_{n+1}}\right) \bar{g}_{n}^{\theta}(i+1) \\
& +\left(\frac{(1+p(\theta) i) q(\theta)}{N_{n+1}}\right) \bar{g}_{n}^{\theta}(i)+q(\theta)\left(\frac{i+2}{N_{n+1}}\right) \bar{g}_{n}^{\theta}(i+2) \\
& +q(\theta) \sum_{j \geq i} \frac{1}{N_{n+1}} \bar{g}_{n}^{\theta}(j)\left(\begin{array}{c}
j \\
i
\end{array}\right) p(\theta)^{i}(1-p(\theta))^{j-i} .
\end{aligned}
$$

Since the probability of duplication step $r=0$, the number of vertices does not increase. Thus, $N_{n}=N_{0}$ and (A.4) can be written as

$$
\begin{aligned}
\bar{g}_{n+1}^{\theta}(i+1)= & \left(1-\frac{1}{N_{0}}(q(\theta)(i+2)+q(\theta)(p(\theta)(i+1)+1))\right) \bar{g}_{n}^{\theta}(i+1) \\
& +\frac{1}{N_{0}}\left((1+p(\theta) i) q(\theta) \bar{g}_{n}^{\theta}(i)+\frac{1}{N_{0}} q(\theta)(i+2) \bar{g}_{n}^{\theta}(i+2)\right) \\
& +\frac{1}{N_{0}} q(\theta) \sum_{j \geq i} \bar{g}_{n}^{\theta}(j)\left(\begin{array}{c}
j \\
i
\end{array}\right) p(\theta)^{i}(1-p(\theta))^{j-i} .
\end{aligned}
$$

It is clear in (A.5) that the vector $\bar{g}_{n+1}^{\theta}$ depends on elements of $\bar{g}_{n}^{\theta}$. In a matrix notation, (A.5) can be expressed as

$$
\bar{g}_{n+1}^{\theta}=\left(I+\frac{1}{N_{0}} L(\theta)\right) \bar{g}_{n}^{\theta}
$$

where $L(\theta)$ is defined as (3.7). 
To prove that $L(\theta)$ is a generator, we need to show that $l_{i i}<0$ and $\sum_{i=1}^{N_{0}} l_{k i}=$ 0 . Accordingly,

$$
\begin{aligned}
\sum_{i=1}^{N_{0}} l_{k i} & =-(q(\theta)(k+1)+q(\theta)(1+p(\theta) k))+(1+p(\theta) k) q(\theta) \\
& +q(\theta) k+q(\theta) \sum_{k \leq i-1}\left(\begin{array}{c}
k \\
i-1
\end{array}\right) p(\theta)^{i-1}(1-p(\theta))^{k-i+1} \\
& =-q(\theta)+q(\theta) \sum_{k \leq i-1}\left(\begin{array}{c}
k \\
i-1
\end{array}\right) p(\theta)^{i-1}(1-p(\theta))^{k-i+1}
\end{aligned}
$$

Let $m=i-1$. Then, A.7 can be rewritten as

$$
\begin{aligned}
\sum_{i=1}^{N_{0}} l_{i k} & =-q(\theta)+q(\theta) \sum_{m=0}^{k}\left(\begin{array}{c}
k \\
m
\end{array}\right) p(\theta)^{m}(1-p(\theta))^{k-m} \\
& =-q(\theta)+q(\theta)(1-p(\theta))^{k} \sum_{m=0}^{k}\left(\begin{array}{c}
k \\
m
\end{array}\right)\left(\frac{p(\theta)}{1-p(\theta)}\right)^{m}
\end{aligned}
$$

Knowing that $\sum_{m=0}^{k}\left(\begin{array}{c}k \\ m\end{array}\right) a^{m}=(1+a)^{k},(\mathrm{~A} .8)$ can be written as

$$
\sum_{i=1}^{N_{0}} l_{i k}=-q(\theta)+q(\theta)(1-p(\theta))^{k}\left(\frac{1}{1-p(\theta)}\right)^{k}=0
$$

Also, it can be shown that $l_{i i}<0$. Since $p(\theta)^{i-1} \leq 1, p(\theta)^{i-1}<1+\frac{2}{i}+$ $p(\theta)+p(\theta)^{i}$. Consequently, $i q(\theta) p(\theta)^{i-1}(1-p(\theta))-q(\theta)(i+2+i p(\theta))<0$. Therefore, $l_{i i}<0$ and the desired result follows. It is straightforward to show that all elements of $B_{N_{0}}(\theta)^{N_{0}}$ are strictly greater than zero. Therefore, $B_{N_{0}}(\theta)$ is irreducible and aperiodic. (Recall that $B_{N_{0}}(\theta)=I+\frac{1}{N_{0}} L(\theta)$.)

\section{A.2 Proof of Theorem 3.5.1}

Define the Lyapunov function $V(x)=\left(x^{\prime} x\right) / 2$ for $x \in \mathbb{R}^{N_{0}}$. Use $\mathbf{E}_{n}$ to denote the conditional expectation with respect to the $\sigma$-algebra $\mathcal{H}_{n}$ generated by 
$\left\{y_{j}, \theta_{j}, \quad j \leq n\right\}$. Then,

$$
\begin{aligned}
& \mathbf{E}_{n}\left\{V\left(\widetilde{g}_{n+1}\right)-V\left(\widetilde{g}_{n}\right)\right\}= \\
& \quad \mathbf{E}_{n}\left\{\widetilde{g}_{n}^{\prime}\left[-\varepsilon \widetilde{g}_{n}+\varepsilon\left(y_{n+1}-\bar{g}\left(\theta_{n}\right)\right)+\bar{g}\left(\theta_{n}\right)-\bar{g}\left(\theta_{n+1}\right)\right]\right\} \\
& \quad+\mathbf{E}_{n}\left\{\left|-\varepsilon \widetilde{g}_{n}+\varepsilon\left(y_{n+1}-\bar{g}\left(\theta_{n}\right)\right)+\bar{g}\left(\theta_{n}\right)-\bar{g}\left(\theta_{n+1}\right)\right|^{2}\right\}
\end{aligned}
$$

where $y_{n+1}$ and $\bar{g}\left(\theta_{n}\right)$ are vectors in $\mathbb{R}^{N_{0}}$ with elements $y_{n}(i)$ and $\bar{g}\left(\theta_{n}, i\right), \quad 1 \leq$ $i \leq N_{0}$, respectively. Due to the Markovian assumption and the structure of the transition matrix of $\theta_{n}$, defined in (3.2),

$$
\begin{aligned}
\mathbf{E}_{n}\left\{\bar{g}\left(\theta_{n}\right)-\bar{g}\left(\theta_{n+1}\right)\right\}=\mathbf{E}\left\{\bar{g}\left(\theta_{n}\right)-\bar{g}\left(\theta_{n+1}\right) \mid \theta_{n}\right\} \\
=\sum_{i=1}^{M} \mathbf{E}\left\{\bar{g}(i)-\bar{g}\left(\theta_{n+1}\right) \mid \theta_{n}=i\right\} I\left\{\theta_{n}=i\right\} \\
=\sum_{i=1}^{M}\left[\bar{g}(i)-\sum_{j=1}^{M} \bar{g}(j) A_{i j}^{\rho}\right] I\left\{\theta_{n}=i\right\} \\
=-\rho \sum_{i=1}^{M} \sum_{j=1}^{M} \bar{g}(j) q_{i j} I\left\{\theta_{n}=i\right\} \\
=O(\rho)
\end{aligned}
$$

where $I(\cdot)$ denotes the indicator function. Similarly, it is easily seen that

$$
\mathbf{E}_{n}\left\{\left|\bar{g}\left(\theta_{n}\right)-\bar{g}\left(\theta_{n+1}\right)\right|^{2}\right\}=O(\rho) .
$$

Using $K$ to denote a generic positive value (with the notation $K K=K$ and $K+K=K$ ), a familiar inequality $a b \leq \frac{a^{2}+b^{2}}{2}$ yields

$$
O(\varepsilon \rho)=O\left(\varepsilon^{2}+\rho^{2}\right) .
$$

Moreover, we have $\left|\widetilde{g}_{n}\right|=\left|\widetilde{g}_{n}\right| \cdot 1 \leq\left(\left|\widetilde{g}_{n}\right|^{2}+1\right) / 2$. Thus,

$$
O(\rho)\left|\widetilde{g}_{n}\right| \leq O(\rho)\left(V\left(\widetilde{g}_{n}\right)+1\right)
$$


Then, detailed estimates lead to

$$
\begin{aligned}
& \mathbf{E}_{n}\left\{\left|-\varepsilon \widetilde{g}_{n}+\varepsilon\left(y_{n+1}-\bar{g}\left(\theta_{n}\right)\right)+\bar{g}\left(\theta_{n}\right)-\bar{g}\left(\theta_{n+1}\right)\right|^{2}\right\} \\
& \quad \leq K \mathbf{E}_{n}\left\{\varepsilon^{2}\left|\widetilde{g}_{n}\right|^{2}+\varepsilon^{2} \mid\left(y_{n+1}-\left.\bar{g}\left(\theta_{n}\right)\right|^{2}+\varepsilon^{2}\left|\widetilde{g}_{n}^{\prime}\left(y_{n+1}-\bar{g}\left(\theta_{n+1}\right)\right)\right|\right.\right. \\
& \left.\quad+\varepsilon\left|\widetilde{g}_{n}^{\prime}\left(\bar{g}\left(\theta_{n}\right)-\bar{g}\left(\theta_{n+1}\right)\right)\right|+\varepsilon\left|\left(y_{n+1}-\bar{g}\left(\theta_{n}\right)\right)^{\prime}\left(\bar{g}\left(\theta_{n}\right)-\bar{g}\left(\theta_{n+1}\right)\right)\right|\right\} \\
& \quad+\mathbf{E}_{n}\left\{\left|\bar{g}\left(\theta_{n}\right)-\bar{g}\left(\theta_{n+1}\right)\right|\right\}^{2} .
\end{aligned}
$$

It follows that

$$
\begin{aligned}
\mathbf{E}_{n}\left\{\mid-\varepsilon \widetilde{g}_{n}\right. & \left.+\varepsilon\left(y_{n+1}-\bar{g}\left(\theta_{n}\right)\right)+\bar{g}\left(\theta_{n}\right)-\left.\bar{g}\left(\theta_{n+1}\right)\right|^{2}\right\} \\
& =O\left(\varepsilon^{2}+\rho^{2}\right)\left(V\left(\widetilde{g}_{n}\right)+1\right) .
\end{aligned}
$$

Furthermore,

$$
\begin{gathered}
\mathbf{E}_{n}\left\{V\left(\widetilde{g}_{n+1}\right)-V\left(\widetilde{g}_{n}\right)\right\}=-2 \varepsilon V\left(\widetilde{g}_{n}\right)+\varepsilon \mathbf{E}_{n}\left\{\widetilde{g}_{n}^{\prime}\left[y_{n+1}-\bar{g}\left(\theta_{n}\right)\right]\right\} \\
+\mathbf{E}_{n}\left\{\widetilde{g}_{n}^{\prime}\left[\bar{g}\left(\theta_{n+1}\right)-\bar{g}\left(\theta_{n}\right)\right]\right\}+O\left(\varepsilon^{2}+\rho^{2}\right)\left(V\left(\widetilde{g}_{n}\right)+1\right) .
\end{gathered}
$$

To obtain the desired bound, define $V_{1}^{\rho}$ and $V_{2}^{\rho}$ as follows:

$$
\begin{aligned}
& V_{1}^{\rho}(\widetilde{g}, n)=\varepsilon \sum_{j=n}^{\infty} \widetilde{g}^{\prime} \mathbf{E}_{n}\left\{y_{j+1}-\bar{g}\left(\theta_{j}\right)\right\}, \\
& V_{2}^{\rho}(\widetilde{g}, n)=\sum_{j=n}^{\infty} \widetilde{g}^{\prime} \mathbf{E}_{n}\left\{\bar{g}\left(\theta_{j}\right)-\bar{g}\left(\theta_{j+1}\right)\right\} .
\end{aligned}
$$

It can be shown that

$$
\begin{aligned}
& \left|V_{1}^{\rho}(\widetilde{g}, n)\right|=O(\varepsilon)(V(\widetilde{g})+1), \\
& \left|V_{2}^{\rho}(\widetilde{g}, n)\right|=O(\rho)(V(\widetilde{g})+1) .
\end{aligned}
$$

Define $W(\widetilde{g}, n)$ as

$$
W(\widetilde{g}, n)=V(\widetilde{g})+V_{1}^{\rho}(\widetilde{g}, n)+V_{2}^{\rho}(\widetilde{g}, n) .
$$

This leads to

$$
\begin{array}{r}
\mathbf{E}_{n}\left\{W\left(\widetilde{g}_{n+1}, n+1\right)-W\left(\widetilde{g}_{n}, n\right)\right\}=\mathbf{E}_{n}\left\{V_{1}^{\rho}\left(\widetilde{g}_{n+1}, n+1\right)-V_{1}^{\rho}\left(\widetilde{g}_{n}, n\right)\right\} \\
+\mathbf{E}_{n}\left\{V\left(\widetilde{g}_{n+1}\right)-V\left(\widetilde{g}_{n}\right)\right\}+\mathbf{E}_{n}\left\{V_{2}^{\rho}\left(\widetilde{g}_{n+1}, n+1\right)-V_{2}^{\rho}\left(\widetilde{g}_{n}, n\right)\right\} .
\end{array}
$$


Moreover,

$$
\begin{aligned}
& \mathbf{E}_{n}\left\{W\left(\widetilde{g}_{n+1}, n+1\right)-W\left(\widetilde{g}_{n}, n\right)\right\} \\
& \quad=-2 \varepsilon V\left(\widetilde{g}_{n}\right)+O\left(\varepsilon^{2}+\rho^{2}\right)\left(V\left(\widetilde{g}_{n}\right)+1\right) .
\end{aligned}
$$

Equation (A.22) can be rewritten as

$$
\begin{aligned}
\mathbf{E}_{n}\left\{W\left(\widetilde{g}_{n+1}, n+1\right)-W\left(\widetilde{g}_{n}, n\right)\right\} \leq & O\left(\varepsilon^{2}+\rho^{2}\right)\left(W\left(\widetilde{g}_{n}, n\right)+1\right) \\
& -2 \varepsilon W\left(\widetilde{g}_{n}, n\right) .
\end{aligned}
$$

If $\varepsilon$ and $\rho$ are chosen small enough, then there exists a small $\lambda$ such that $-2 \varepsilon+$ $O\left(\rho^{2}\right)+O\left(\varepsilon^{2}\right) \leq-\lambda \varepsilon$. Therefore, A.23 can be rearranged as

$$
\mathbf{E}_{n}\left\{W\left(\widetilde{g}_{n+1}, n+1\right)\right\} \leq(1-\lambda \varepsilon) W\left(\widetilde{g}_{n}, n\right)+O\left(\varepsilon^{2}+\rho^{2}\right) .
$$

Taking expectation of both sides yields

$$
\mathbf{E}\left\{W\left(\widetilde{g}_{n+1}, n+1\right)\right\} \leq(1-\lambda \varepsilon) \mathbf{E}\left\{W\left(\widetilde{g}_{n}, n\right)\right\}+O\left(\varepsilon^{2}+\rho^{2}\right) .
$$

Iterating on $\mathrm{A} .25$ then results

$$
\begin{aligned}
\mathbf{E}\left\{W\left(\widetilde{g}_{n+1}, n+1\right)\right\} \leq & (1-\lambda \varepsilon)^{n-N_{\rho}} \mathbf{E}\left\{W\left(\widetilde{g}_{N_{\rho}}, N_{\rho}\right)\right\} \\
& +\sum_{j=N_{\rho}}^{n} O\left(\varepsilon^{2}+\rho^{2}\right)(1-\lambda \varepsilon)^{j-N_{\rho}}
\end{aligned}
$$

As the result,

$$
\begin{gathered}
\mathbf{E}\left\{W\left(\widetilde{g}_{n+1}, n+1\right)\right\} \leq(1-\lambda \varepsilon)^{n-N_{\rho}} \mathbf{E}\left\{W\left(\widetilde{g}_{N_{\rho}}, N_{\rho}\right)\right\} \\
+O\left(\varepsilon+\rho^{2} / \varepsilon\right) .
\end{gathered}
$$

If $n$ is large enough, one can approximate $(1-\lambda \varepsilon)^{n-N_{\rho}}=O(\varepsilon)$. Therefore,

$$
\mathbf{E}\left\{W\left(\widetilde{g}_{n+1}, n+1\right)\right\} \leq O\left(\varepsilon+\frac{\rho^{2}}{\varepsilon}\right)
$$

Finally, using (A.19) and replacing $W\left(\widetilde{g}_{n+1}, n+1\right)$ with $V\left(\widetilde{g}_{n+1}\right)$, we obtain

$$
\mathbf{E}\left\{V\left(\widetilde{g}_{n+1}\right)\right\} \leq O\left(\rho+\varepsilon+\frac{\rho^{2}}{\varepsilon}\right) .
$$




\section{A.3 Sketch of the Proof of Theorem 3.5.2}

The proof uses weak convergence techniques [85].

Let $Z_{k}$ and $Z$ be $\mathbb{R}^{r}$-valued random vectors. We say $Z_{k}$ converges weakly to $Z$ $\left(Z_{n} \Rightarrow Z\right)$ if for any bounded and continuous function $f(\cdot), E f\left(Z_{k}\right) \rightarrow E f(Z)$ as $k \rightarrow \infty$. The sequence $\left\{Z_{k}\right\}$ is tight if for each $\eta>0$, there is a compact set $K_{\eta}$ such that $P\left(Z_{n} \in K_{\eta}\right) \geq 1-\eta$ for all $k$. The definitions of weak convergence and tightness extend to random elements in more general metric spaces. On a complete separable metric space, tightness is equivalent to relative compactness, which is known as the Prohorov's Theorem [85]. By virtue of this theorem, we can extract convergent subsequences when tightness is verified. In what follows, we use a martingale problem formulation to establish the desired weak convergence. This usually requires to first prove tightness. The limit process is then characterized using certain operator related to the limit process. We refer the reader to [167, Chapter 7] for further details on weak convergence and related matters.

Since the proof is similar to [260, Theorem 4.5], we only indicate the main steps in what follows and omit most of the verbatim details.

Step 1: First, we show that the two component process $\left(\widehat{g}^{\varepsilon}(\cdot), \theta^{\varepsilon}(\cdot)\right)$ is tight in $D\left([0, T]: \mathbb{R}^{N_{0}} \times M\right)$, which is the space of functions defined on $[0, T]$ taking values in $\mathbb{R}^{N_{0}} \times M$ that are right continuous, have left limits, and endowed with the Skorohod topology. Using techniques similar to [262, Theorem 4.3], it can be shown that $\theta^{\varepsilon}(\cdot)$ converges weakly to a continuous-time Markov chain generated by $Q$. Thus, we mainly need to consider $\widehat{g}^{\varepsilon}(\cdot)$. We show that

$$
\lim _{\Delta \rightarrow 0} \limsup _{\varepsilon \rightarrow 0} \mathbf{E}\left[\sup _{0 \leq s \leq \Delta} \mathbf{E}_{t}^{\varepsilon}\left|\widehat{g}^{\varepsilon}(t+s)-\widehat{g}^{\varepsilon}(t)\right|^{2}\right]=0
$$

where $\mathbf{E}_{t}^{\varepsilon}$ denotes the conditioning on the past information up to $t$. Then, the tightness follows from the criterion [165, p. 47].

Step 2: Since $\left(\widehat{g}^{\varepsilon}(\cdot), \theta^{\varepsilon}(\cdot)\right)$ is tight, we can extract weakly convergent subsequence according to the Prohorov theorem; see [167]. To determine the limit, we show that $\left(\widehat{g}^{\varepsilon}(\cdot), \theta^{\varepsilon}(\cdot)\right)$ is a solution of the martingale problem with operator $L_{0}$. For each $i \in \mathcal{M}$ and continuously differential function with compact support $f(\cdot, i)$, the operator is given by

$$
L_{0} f(\widehat{g}, i)=\nabla f^{\prime}(\widehat{g}, i)[-\widehat{g}+\bar{g}(i)]+\sum_{j \in \mathcal{M}} q_{i j} f(\widehat{g}, j), i \in \mathcal{M}
$$

We can further demonstrate the martingale problem with operator $L_{0}$ has a unique 
weak solution. Thus, the desired convergence property follows.

\section{A.4 Sketch of the Proof of Theorem 3.5.3}

The proof comprises of four steps as described below:

Step 1: First, note

$$
\nu_{n+1}=\nu_{n}-\varepsilon \nu_{n}+\sqrt{\varepsilon}\left(y_{n+1}-\mathbf{E} \bar{g}\left(\theta_{n}\right)\right)+\frac{\mathbf{E}\left[\bar{g}\left(\theta_{n}\right)-\bar{g}\left(\theta_{n+1}\right]\right.}{\sqrt{\varepsilon}} .
$$

The approach is similar to that of [260, Theorem 5.6]. Therefore, we will be brief.

Step 2: Define the operator

$$
\mathcal{L} f(\nu, i)=-\nabla f^{\prime}(\nu, i) \nu+\frac{1}{2} \operatorname{tr}\left[\nabla^{2} f(\nu, i) \Sigma(i)\right]+\sum_{j \in \mathcal{M}} q_{i j} f(\nu, j), i \in \mathcal{M},
$$

for function $f(\cdot, i)$ with compact support that has continuous partial derivatives with respect to $\nu$ up to the second order. It can be shown that the associated martingale problem has a unique solution (in the sense of in distribution).

Step 3: It is natural now to work with a truncated process. For a fixed, but otherwise arbitrary $r_{1}>0$, define a truncation function

$$
q^{r_{1}}(x)= \begin{cases}1, & \text { if } x \in S^{r_{1}} \\ 0, & \text { if } x \in \mathbb{R}^{N_{0}}-S^{r_{1}}\end{cases}
$$

where $S^{r_{1}}=\left\{x \in \mathbb{R}^{N_{0}}:|x| \leq r_{1}\right\}$. Then, we obtain the truncated iterates

$$
\begin{aligned}
\nu_{n+1}^{r_{1}}= & \nu_{n}^{r_{1}}-\varepsilon \nu_{n}^{r_{1}} q^{r_{1}}\left(\nu_{n}^{r_{1}}\right)+\sqrt{\varepsilon}\left(y_{n+1}-\mathbf{E} \bar{g}\left(\theta_{n}\right)\right) \\
& +\frac{\mathbf{E}\left[\bar{g}\left(\theta_{n}\right)-\bar{g}\left(\theta_{n+1}\right]\right.}{\sqrt{\varepsilon}} q^{r_{1}}\left(\nu_{n}^{r_{1}}\right) .
\end{aligned}
$$

Define $\nu^{\varepsilon, r_{1}}(t)=\nu_{n}^{r_{1}}$ for $t \in[\varepsilon n, \varepsilon n+\varepsilon)$. Then, $\nu^{\varepsilon, r_{1}}(\cdot)$ is an $r$-truncation of $\nu^{\varepsilon}(\cdot)$; see [167, p. 284] for a definition. We then show the truncated process $\left(\nu^{\varepsilon, r_{1}}(\cdot), \theta^{\varepsilon}(\cdot)\right)$ is tight. Moreover, by Prohorov's theorem, we can extract a convergent subsequence with limit $\left(\nu^{r_{1}}(\cdot), \theta(\cdot)\right)$ such that the limit $\left(\nu^{r_{1}}(\cdot), \theta(\cdot)\right)$ is the solution of the martingale problem with operator $\mathcal{L}^{r_{1}}$ defined by

$$
\mathcal{L}^{r_{1}} f^{r_{1}}(\nu, i)=-\nabla \prime f^{r_{1}}(\nu, i) \nu+\frac{1}{2} \operatorname{tr}\left[\nabla^{2} f^{r_{1}}(\nu, i) \Sigma(i)\right]+\sum_{j \in \mathcal{M}} q_{i j} f^{r_{1}}(\nu, j)
$$


for $i \in \mathcal{M}$, where $f^{r_{1}}(\nu, i)=f(\nu, i) q^{r_{1}}(\nu)$.

Step 4: Letting $r_{1} \rightarrow \infty$, we show that the un-truncated process also converges and the limit, denoted by $(\nu(\cdot), \theta(\cdot))$, is precisely the martingale problem with operator $\mathcal{L}$ defined in (A.33). The limit covariance can further be evaluated as in [260, Lemma 5.2]. 


\section{Appendix B}

\section{Appendix to Chapter 5}

This appendix starts with a formal definition of differential inclusions that capture the asymptotic local behavior of individual agents that follow the algorithms of $\$ 5.3$.

Definition B.1. Differential inclusions are generalizations of ordinary differential equations. In this monograph, we consider differential inclusions of the form

$$
\frac{d}{d t} X \in \mathcal{F}(X)
$$

where $X \in \mathbb{R}^{r}$ and $\mathcal{F}: \mathbb{R}^{r} \rightarrow \mathbb{R}^{r}$ is a Marchaud map [14]. That is: (i) the graph and domain of $\mathcal{F}$ are nonempty and closed, (ii) the values $\mathcal{F}(X)$ are convex, and (iii) the growth of $\mathcal{F}$ is linear, i.e., there exists $\eta>0$ such that for every $X \in \mathbb{R}^{r}$ :

$$
\sup _{Y \in \mathcal{F}(X)}\|Y\| \leq \eta(1+\|X\|)
$$

where $\|\cdot\|$ denotes any norm on $\mathbb{R}^{r}$.

The rest of this appendix presents details on the proofs of theorems presented in $\$ 5.4$. The key tools we use are weak convergence and Lyapunov stability methods. We refer to [85, 167] for excellent textbook treatments of weak convergence theory. 


\section{B.1 Proof of Theorem 5.4.2}

Close scrutiny of the action selection strategy (5.12) reveals that $\left\{\mathrm{a}_{k}^{n}, R_{k}^{n}\right\}$ is a Markov chain with the transition matrix $P\left(R^{n}\right)=\left[P_{i j}\left(R^{n}\right)\right]$ given by

$$
\begin{aligned}
P_{i j}\left(R_{k}^{n}\right) & =\mathbb{P}\left(\mathrm{a}_{k}^{n}=j \mid \mathrm{a}_{k-1}^{n}=i, R_{k}^{n}\right) \\
& = \begin{cases}\frac{1}{\mu^{n}}\left|r_{k}^{n}(i, j)\right|^{+}, & i \neq j, \\
1-\sum_{j \neq i} P_{i j}\left(R_{k}^{n}\right), & i=j .\end{cases}
\end{aligned}
$$

That is, the transition matrix is a function of the regret matrix $R^{n}$. It is straightforward to show that the transition matrix $P\left(R^{n}\right)$ satisfies the following conditions:

(i) it is continuous in $R^{n}$;

(ii) for each $R^{n}$, it is irreducible and aperiodic.

Condition (ii) essentially indicates that the Markov chain is ergodic in the sense that there is a unique stationary distribution $\psi^{n}\left(R^{n}\right)=\left(\psi_{1}^{n}\left(R^{n}\right), \ldots, \psi_{A^{k}}^{n}\left(R^{n}\right)\right)$ such that $\left[P\left(R^{n}\right)\right]^{n} \rightarrow \mathbf{1} \psi^{n}\left(R^{n}\right)$, a matrix with identical rows consisting of the stationary distribution as $n \rightarrow \infty$. (The convergence in fact takes place exponentially fast.)

Further, the sequence of opponents' action profile $\left\{\mathbf{a}_{k}^{-n}\right\}$ is independent of $\left\{\mathrm{a}_{k}^{n}\right\}$, and satisfies the following condition:

$$
d\left[\frac{1}{n} \sum_{\ell=m}^{n+m-1} \mathbf{E}_{m}\left\{B^{n}\left(i, \mathbf{a}_{\ell}^{-n}\right)\right\}, \mathcal{S}^{n}(i)\right] \rightarrow 0 \text { in probability }
$$

where $d[\cdot, \cdot]$ denotes the usual distance function, $\mathbf{E}_{m}$ denotes conditional expectation given the $\sigma$-algebra generated by $\left\{R_{\ell}^{n}, \mathrm{a}_{\ell-1}^{n}, \mathbf{a}_{\ell-1}^{-n}: \ell \leq m\right\}$, and $\mathcal{S}^{n}(i)=$ $\left[\mathcal{S}_{p q}^{n}(i)\right]$ is a set of $A^{n} \times A^{n}$ matrices, where

$$
\mathcal{S}_{p q}^{n}(i)= \begin{cases}\left\{U^{n}\left(q, \mathbf{p}^{-n}\right)-U^{n}\left(i, \mathbf{p}^{-n}\right) ; \mathbf{p}^{-n} \in \Delta \mathcal{A}^{-n}\right\} & p=i \\ 0 & p \neq i\end{cases}
$$

It is then straight forward to show that, for any stationary distribution $\boldsymbol{\psi}^{n}\left(R^{n}\right)$, the set

$$
H^{n}\left(R^{n}\right)=\sum_{i \in \mathcal{A}^{n}} \psi_{i}^{n}\left(R^{n}\right) \mathcal{S}^{n}(i)
$$

is closed, convex, and upper semi-continuous; see [167, pp.108-109]. 
To proceed, recall the continuous-time interpolated process $R^{n, \varepsilon}(t)=R_{k}^{n}$ for $t \in[k \varepsilon,(k+1) \varepsilon)$. We first prove the tightness . Consider (5.14) for the sequence of $\left(A^{k}\right)^{2}$-valued vectors resulted after rearranging the elements of the regret matrix $R^{n}$ into a column vector. Noting the boundedness of payoff functions, and using Hölder's and Gronwall's inequalities, for any $0<T<\infty$, we obtain

$$
\sup _{k \leq T / \varepsilon} \mathbf{E}\left\|R_{k}^{n}\right\|^{2}<\infty
$$

where in the above and hereafter $t / \varepsilon$ is understood to be the integer part of $t / \varepsilon$ for each $t>0$. Next, considering $R^{n, \varepsilon}(\cdot)$, for any $t, s>0, \delta>0$, and $s<\delta$, it is fairly easy to verify that

$$
R^{n, \varepsilon}(t+s)-R^{n, \varepsilon}(t)=\varepsilon \sum_{\ell=t / \varepsilon}^{(t+s) / \varepsilon-1}\left[B^{n}\left(\mathrm{a}_{\ell}^{n}, \mathcal{A}_{\ell}^{-n}\right)-R_{\ell}^{n}\right]
$$

As a result,

$$
\lim _{\delta \rightarrow 0} \limsup _{\mu \rightarrow 0}\left\{\mathbf{E}\left[\sup _{0 \leq s \leq \delta} \mathbf{E}_{t}^{\varepsilon}\left\|R^{n, \varepsilon}(t+s)-R^{n, \varepsilon}(t)\right\|^{2}\right]\right\}=0 .
$$

Thus, $R^{n, \varepsilon}(\cdot)$ is tight in the space of functions that are defined in $[0, \infty)$ taking values in $\mathbb{R}^{\left(A^{k}\right)^{2}}$; see [167, Chapter 7] or [165, Theorem 3, p. 47].

By Prohorov theorem [167], one can extract a convergent subsequence. For notational simplicity, we still denote this convergent subsequence by $R^{n, \varepsilon}(\cdot)$ with limit $R^{n}(\cdot)$. Thus, $R^{n, \varepsilon}(\cdot)$ converges weakly to $R^{n}(\cdot)$. By Skorohod representation theorem [167], with a slight abuse of notation, one can assume $R^{n, \varepsilon}(\cdot) \rightarrow R^{n}(\cdot)$ with probability one and the convergence is uniform on any finite interval. Next, using martingale averaging methods, we need to characterize the limit process. Normally, one uses a smooth function $f(\cdot)$ with compact support to carry out the analysis. Here, for simplicity, we suppress the dependence of $f(\cdot)$ and proceed directly; see [166] for a similar argument. Choose a sequence of integers $\left\{k_{\varepsilon}\right\}$ such that $k_{\varepsilon} \rightarrow \infty$ as $\varepsilon \rightarrow 0$, but $\delta_{\varepsilon}=\varepsilon k_{\varepsilon} \rightarrow 0$. Note

$$
\begin{aligned}
R^{n, \varepsilon}(t+s)-R^{n, \varepsilon}(t)= & \sum_{\ell: \ell \delta_{\varepsilon}=t}^{t+s} \delta_{\varepsilon} \sum_{i \in \mathcal{A}^{n}} \frac{1}{k_{\varepsilon}} \sum_{\tau=\ell k_{\varepsilon}}^{\ell k_{\varepsilon}+k_{\varepsilon}-1} B^{n}\left(i, \mathbf{a}_{\tau}^{-n}\right) I\left\{\mathrm{a}_{\tau}^{n}=i\right\} \\
& -\sum_{\ell: \ell \delta_{\varepsilon}=t}^{t+s} \delta_{\varepsilon} \frac{1}{k_{\varepsilon}} \sum_{\tau=\ell k_{\varepsilon}}^{\ell k_{\varepsilon}+k_{\varepsilon}-1} R_{\tau}^{n}
\end{aligned}
$$


where $\sum_{\ell: \ell \delta_{\varepsilon}=t}^{t+s}$ denotes the sum over integers $\ell$ in the range $t \leq \ell \delta_{\varepsilon} \leq t+s$.

We shall use the techniques in [167, Chapter 8] to prove the desired result. Let $h(\cdot)$ be any bounded and continuous function, $t, s>0, \kappa_{0}$ be an arbitrary positive integer, and $t_{\iota} \leq t$ for all $\iota \leq \kappa_{0}$. It is readily seen that, by virtue of the weak convergence and the Skorohod representation, as $\varepsilon \rightarrow 0$,

$$
\begin{aligned}
\mathbf{E} h\left(R^{n, \varepsilon}\left(t_{\iota}\right): \iota \leq \kappa_{0}\right)\left[R^{n, \varepsilon}(t+s)-R^{n, \varepsilon}(t)\right] \\
\quad \rightarrow \mathbf{E} h\left(R^{n}\left(t_{\iota}\right): \iota \leq \kappa_{0}\right)\left[R^{n}(t+s)-R^{n}(t)\right] .
\end{aligned}
$$

By using the technique of stochastic approximation (see, e.g., [167, Chapter 8]), we also have

$$
\begin{aligned}
& \mathbf{E} h\left(R^{n, \varepsilon}\left(t_{\iota}\right): \iota \leq \kappa_{0}\right) \sum_{\ell \delta_{\varepsilon}=t}^{t+s} \delta_{\varepsilon} \frac{1}{k_{\varepsilon}} \sum_{\tau=\ell k_{\varepsilon}}^{\ell k_{\varepsilon}+k_{\varepsilon}-1} R_{\tau}^{n} \\
& \rightarrow \mathbf{E} h\left(R^{k}\left(t_{\iota}\right): \iota \leq \kappa_{0}\right)\left[\int_{t}^{t+s} R^{n}(u) d u\right] \text { as } \varepsilon \rightarrow 0 .
\end{aligned}
$$

Moreover, by the independence of $\mathrm{a}_{k}^{n}$ and $\mathbf{a}_{k}^{-n}$,

$$
\begin{gathered}
\lim _{\varepsilon \rightarrow 0} \mathbf{E} h\left(R^{n, \varepsilon}\left(t_{\iota}\right): \iota \leq \kappa_{0}\right) \\
\times\left[\sum_{\ell \delta_{\varepsilon}=t}^{t+s} \delta_{\varepsilon} \sum_{i \in \mathcal{A}^{n}} \frac{1}{k_{\varepsilon}} \sum_{\tau=\ell k_{\varepsilon}}^{\ell k_{\varepsilon}+k_{\varepsilon}-1} B^{n}\left(i, \mathbf{a}_{\tau}^{-n}\right) I\left\{\mathbf{a}_{\tau}^{n}=i\right\}\right] \\
=\lim _{\varepsilon \rightarrow 0} \mathbf{E} h\left(R^{n, \varepsilon}\left(t_{\iota}\right): \iota \leq \kappa_{0}\right) \\
\times\left[\sum_{\ell \delta_{\varepsilon}=t}^{t+s} \delta_{\varepsilon} \sum_{i \in \mathcal{A}^{n}} \frac{1}{k_{\varepsilon}} \sum_{\tau=\ell k_{\varepsilon}}^{\ell k_{\varepsilon}+k_{\varepsilon}-1} \mathbf{E}_{\ell k_{\varepsilon}}\left\{B^{n}\left(i, \mathbf{a}_{\tau}^{-n}\right)\right\} \mathbf{E}_{\ell k_{\varepsilon}} I\left\{\mathbf{a}_{\tau}^{n}=i\right\}\right] \\
=\lim _{\varepsilon \rightarrow 0} \mathbf{E} h\left(R^{n, \varepsilon}\left(t_{\iota}\right): \iota \leq \kappa_{0}\right)\left[\sum_{\ell \delta_{\varepsilon}=t}^{t+s} \delta_{\varepsilon} \sum_{i \in \mathcal{A}^{n}} \frac{1}{k_{\varepsilon}} \sum_{\tau=\ell k_{\varepsilon}}^{\ell k_{\varepsilon}+k_{\varepsilon}-1} \sum_{j \in \mathcal{A}^{n}} I\left\{\mathbf{a}_{\ell k_{\varepsilon}}^{n}=j\right\}\right. \\
\times\left[\mathbf{E}_{\ell k_{\varepsilon}}\left\{B^{n}\left(i, \mathbf{a}_{\tau}^{-n}\right)\right\} \varphi_{i}\left(R_{\ell n_{\varepsilon}}^{n}\right)+\mathbf{E}_{\ell k_{\varepsilon}}\left\{B^{n}\left(i, \mathbf{a}_{\tau}^{-n}\right)\right\}\right. \\
\left.\left.\times\left[P_{j i}^{\left\{\tau-\ell k_{\varepsilon}\right\}}\left(R_{\ell k_{\varepsilon}}^{n}\right)-\varphi_{i}\left(R_{\ell k_{\varepsilon}}^{n}\right)\right]\right]\right]
\end{gathered}
$$

In (B.13), $P_{j i}^{\left\{\tau-\ell k_{\varepsilon}\right\}}\left(R^{n}\right)$ denotes the $\left(\tau-\ell k_{\varepsilon}\right)$-step transition probability. Except the indicator function on the 1.h.s. of (B.14), the rest of the terms in the summand 
are independent of $j$, therefore,

$$
\begin{gathered}
\sum_{j \in \mathcal{A}^{n}} I\left\{\mathrm{a}_{\ell k_{\varepsilon}}^{n}=j\right\} \mathbf{E}_{\ell k_{\varepsilon}}\left\{B^{n}\left(i, \mathbf{a}_{\tau}^{-n}\right)\right\} \varphi_{i}\left(R_{\ell k_{\varepsilon}}^{n}\right) \\
=\mathbf{E}_{\ell k_{\varepsilon}}\left\{B^{n}\left(i, \mathbf{a}_{\tau}^{-n}\right)\right\} \varphi_{i}\left(R_{\ell k_{\varepsilon}}^{n}\right) .
\end{gathered}
$$

Recalling the properties of the transition matrix $P\left(R^{n}\right)$, regardless of the choice of $j$, as $\varepsilon \rightarrow 0$ and $\tau-\ell k_{\varepsilon} \rightarrow \infty$, for each fixed $R^{n}$,

$$
\left[P_{j i}^{\left\{\tau-\ell k_{\varepsilon}\right\}}\left(R^{n}\right)-\varphi_{i}\left(R^{n}\right)\right] \rightarrow 0
$$

exponentially fast. As the result, as $\varepsilon \rightarrow 0$,

$$
\begin{aligned}
& \mathbf{E} h\left(R^{n, \varepsilon}\left(t_{\iota}\right): \iota \leq \kappa_{0}\right) \\
& \times\left[\sum_{\ell \delta_{\varepsilon}=t}^{t+s} \delta_{\varepsilon} \sum_{i \in \mathcal{A}^{n}} \frac{1}{k_{\varepsilon}} \sum_{k=\ell k_{\varepsilon}}^{\ell k_{\varepsilon}+k_{\varepsilon}-1} \sum_{j \in \mathcal{A}^{n}} I\left\{\mathbf{a}_{\ell k_{\varepsilon}}^{n}=j\right\} \mathbf{E}_{\ell k_{\varepsilon}}\left\{B^{n}\left(i, \mathbf{a}_{\tau}^{-n}\right)\right\}\right. \\
& \left.\quad \times\left[P_{j i}^{\left\{\tau-\ell k_{\varepsilon}\right\}}\left(R_{\ell k_{\varepsilon}}^{n}\right)-\varphi_{i}\left(R_{\ell k_{\varepsilon}}^{n}\right)\right]\right] \rightarrow 0 \text { in probability. }
\end{aligned}
$$

Therefore,

$$
\begin{array}{rl}
\lim _{\varepsilon \rightarrow 0} \mathbf{E} & h\left(R^{n, \varepsilon}\left(t_{\iota}\right): \iota \leq \kappa_{0}\right) \\
\times & {\left[\sum_{\ell \delta_{\varepsilon}=t}^{t+s} \delta_{\varepsilon} \sum_{i \in \mathcal{A}^{n}} \frac{1}{k_{\varepsilon}} \sum_{\tau=\ell k_{\varepsilon}}^{\ell k_{\varepsilon}+k_{\varepsilon}-1} B^{n}\left(i, \mathbf{a}_{\tau}^{-n}\right) I\left\{\mathbf{a}_{\tau}^{n}=i\right\}\right]} \\
=\lim _{\varepsilon \rightarrow 0} \mathbf{E} h\left(R^{n, \varepsilon}\left(t_{\iota}\right): \iota \leq \kappa_{0}\right) \\
\times\left[\sum_{\ell \delta_{\varepsilon}=t}^{t+s} \delta_{\varepsilon} \sum_{i \in \mathcal{A}^{n}} \frac{1}{k_{\varepsilon}} \sum_{\tau=\ell k_{\varepsilon}}^{\ell k_{\varepsilon}+k_{\varepsilon}-1} \mathbf{E}_{\ell k_{\varepsilon}}\left\{B^{n}\left(i, \mathbf{a}_{\tau}^{-n}\right)\right\} \varphi_{i}\left(R_{\ell n_{\varepsilon}}^{n}\right)\right] .
\end{array}
$$

Using (B.4) together with (B.17), we obtain that, as $\varepsilon \rightarrow 0$,

$$
\begin{gathered}
\mathbf{E} h\left(R^{n, \varepsilon}\left(t_{\iota}\right): \iota \leq \kappa_{0}\right)\left[\sum_{\ell \delta_{\varepsilon}=t}^{t+s} \delta_{\varepsilon} \sum_{i \in \mathcal{A}^{n}} \frac{1}{k_{\varepsilon}} \sum_{\tau=\ell k_{\varepsilon}}^{\ell k_{\varepsilon}+k_{\varepsilon}-1} B^{n}\left(i, \mathbf{a}_{\tau}^{-n}\right) I\left\{\mathbf{a}_{\tau}^{n}=i\right\}\right] \\
\rightarrow \mathbf{E} h\left(R^{n, \varepsilon}\left(t_{\iota}\right): \iota \leq \kappa_{0}\right)\left[\int_{t}^{t+s} \sum_{i \in \mathcal{A}^{n}} \varphi_{i}\left(R^{n}(u)\right) \mathcal{S}^{n}(i) d u\right] .
\end{gathered}
$$


Finally, combining ( $(\underline{B .12})$ and (B.18) , and using (B.6) yields

$$
\frac{d R^{n}(t)}{d t} \in H^{n}\left(R^{n}\right)-R^{n}(t)
$$

as desired.

\section{B.2 Proof of Theorem 5.4.5}

Define the Lyapunov function

$$
V\left(R^{n}\right)=\frac{1}{2}\left[\operatorname{dist}\left[R^{n}, \mathbb{R}^{-}\right]\right]^{2}=\frac{1}{2} \sum_{i, j \in \mathcal{A}^{n}}\left[\left|r^{n}(i, j)\right|^{+}\right]^{2} .
$$

For the sake of notational convenience, we drop the $R^{n}$ dependency of the invariant measure, and denote it simply by $\boldsymbol{\psi}^{n}$ hereafter.

Case I: Taking the time-derivative of (B.20), and substituting for $d r^{n}(i, j) / d t$ from (5.44)- (5.45), we obtain

$$
\begin{aligned}
\frac{d}{d t} V\left(R^{n}\right)= & \sum_{i, j \in \mathcal{A}^{n}}\left|r^{n}(i, j)\right|^{+} \cdot \frac{d}{d t} r^{n}(i, j) \\
= & \sum_{i, j \in \mathcal{A}^{n}}\left|r^{n}(i, j)\right|^{+}\left[\left[U^{n}\left(j, \mathbf{p}^{-n}\right)-U^{n}\left(i, \mathbf{p}^{-n}\right)\right] \psi_{i}^{n}-r^{n}(i, j)\right] \\
= & \underbrace{\sum_{i, j \in \mathcal{A}^{n}}\left|r^{n}(i, j)\right|^{+}\left[U^{n}\left(j, \mathbf{p}^{-n}\right)-U^{n}\left(i, \mathbf{p}^{-n}\right)\right] \psi_{i}^{n}}_{=0 \text { (See Lemma[B.1below) }} \\
& -\sum_{i, j \in \mathcal{A}^{n}}\left|r^{n}(i, j)\right|^{+} r^{n}(i, j) \\
= & -V\left(R^{n}\right) .
\end{aligned}
$$

In the last equality we used

$$
\sum_{i, j \in \mathcal{A}^{n}}\left|r^{n}(i, j)\right|^{+} r^{n}(i, j)=\sum_{i, j \in \mathcal{A}^{n}}\left[\left|r^{n}(i, j)\right|^{+}\right]^{2}=V\left(R^{n}\right) .
$$

This completes the proof.

The following lemma shows that the first term in the r.h.s. of (B.21) equals zero. 
Lemma B.1. Let $\mathbf{p}^{-n}$ denote a probability measure over the joint action space $\mathcal{A}^{-n}$ of all agents excluding agent $k$. Then,

$$
\sum_{i, j \in \mathcal{A}^{n}}\left|r^{n}(i, j)\right|^{+}\left[U^{n}\left(j, \mathbf{p}^{-n}\right)-U^{n}\left(i, \mathbf{p}^{-n}\right)\right] \psi_{i}^{n}=0
$$

where $\psi^{n}=\left(\psi_{1}^{n}, \ldots, \psi_{A^{n}}^{n}\right)$ represents the invariant measure of transition probabilities (5.12).

Proof. Separating the summation over the two terms in (B.23) yields

$$
\begin{aligned}
& \sum_{i, j \in \mathcal{A}^{n}}\left|r^{n}(i, j)\right|^{+} U^{n}\left(j, \mathbf{p}^{-n}\right) \psi_{i}^{n}-\sum_{i, j \in \mathcal{A}^{n}}\left|r^{n}(i, j)\right|^{+} U^{n}\left(i, \mathbf{p}^{-n}\right) \psi_{i}^{n} \\
& =\sum_{j, i \in \mathcal{A}^{n}}\left|r^{n}(j, i)\right|^{+} U^{n}\left(i, \mathbf{p}^{-n}\right) \psi_{j}^{n}-\sum_{i, j \in \mathcal{A}^{n}}\left|r^{n}(i, j)\right|^{+} U^{n}\left(i, \mathbf{p}^{-n}\right) \psi_{i}^{n} \\
& =\sum_{i \in \mathcal{A}^{n}} U^{n}\left(i, \mathbf{p}^{-n}\right)\left[\sum_{j \in \mathcal{A}^{n}} \psi_{j}^{n}\left|r^{n}(j, i)\right|^{+}-\sum_{j \in \mathcal{A}^{n}} \psi_{i}^{n}\left|r^{n}(i, j)\right|^{+}\right] .
\end{aligned}
$$

In the second line we used $\sum_{i, j} a_{i j}=\sum_{j, i} a_{j i}$, and in the last line we changed the order of summation both applied to the first term. Finally, applying $\left|r^{n}(i, i)\right|^{+}=$ 0 , for all $i \in \mathcal{A}^{n}$, and substituting (5.42) into (B.24) completes the proof.

Case II: Define the Lyapunov function

$$
\begin{aligned}
V\left(R^{L, n}, R^{G, n}\right) & =\left[\operatorname{dist}\left[R^{L, n}+R^{G, n}, \mathbb{R}^{-}\right]\right]^{2} \\
& =\sum_{i, j \in \mathcal{A}^{n}}\left[\left|r^{L, n}(i, j)+r^{G, n}(i, j)\right|^{+}\right]^{2}
\end{aligned}
$$

Taking the time-derivative of (B.25) yields

$$
\begin{aligned}
\frac{d}{d t} V\left(R^{L, n}, R^{G, n}\right)=2 \sum_{i, j \in \mathcal{A}^{n}} & \left|r^{L, n}(i, j)+r^{G, n}(i, j)\right|^{+} \\
\times & {\left[\frac{d}{d t} r^{L, n}(i, j)+\frac{d}{d t} r^{G, n}(i, j)\right] . }
\end{aligned}
$$


Next, replacing for $d r_{i j}^{L, n} / d t$ and $d r_{i j}^{G, n} / d t$ from (5.48)-(5.49),

$$
\begin{aligned}
\frac{d}{d t} V\left(R^{L, n}, R^{G, n}\right)=2 \sum_{i, j \in \mathcal{A}^{n}} \mid & r^{L, n}(i, j)+\left.r^{G, n}(i, j)\right|^{+} \\
\times[ & {\left[U_{L}^{n}\left(j, \boldsymbol{\nu}^{-n}\right)-U_{L}^{n}\left(i, \boldsymbol{\nu}^{-n}\right)\right] \sigma_{i}^{n} } \\
& +\left[U_{G, t}^{n}(j)-U_{G, t}^{n}(i)\right] \sigma_{i}^{n} \\
& \left.-\left[r^{L, n}(i, j)+r^{G, n}(i, j)\right]\right] .
\end{aligned}
$$

Substituting for $\sigma^{n}$ from (5.46) in (B.27) yields

$$
\begin{aligned}
\frac{d}{d t} V( & \left.R^{L, n}, R^{G, n}\right)=2(1-\delta) \sum_{i, j} \psi_{i}^{n}\left|r^{L, n}(i, j)+r^{G, n}(i, j)\right|^{+} \\
\times & {\left[U_{L}^{n}\left(j, \boldsymbol{\nu}^{-n}\right)+U_{G, t}^{n}(j)-\left[U_{L}^{n}\left(i, \boldsymbol{\nu}^{-n}\right)+U_{G, t}^{n}(i)\right]\right] } \\
& +\frac{2 \delta}{A^{n}} \sum_{i, j \in \mathcal{A}^{n}}\left|r^{L, n}(i, j)+r^{G, n}(i, j)\right|^{+} \\
\times & {\left[U_{L}^{n}\left(j, \boldsymbol{\nu}^{-n}\right)+U_{G, t}^{n}(j)-\left[U_{L}^{n}\left(i, \boldsymbol{\nu}^{-n}\right)+U_{G, t}^{n}(i)\right]\right] } \\
& \quad-2 \sum_{i, j \in \mathcal{A}^{n}}\left|r^{L, n}(i, j)+r^{G, n}(i, j)\right|^{+}\left[r^{L, n}(i, j)+r^{G, n}(i, j)\right] .
\end{aligned}
$$

Similar to Lemma B.1, it is straightforward to show that the first term in the r.h.s. of (B.28) is zero. Therefore,

$$
\begin{aligned}
& \frac{d}{d t} V\left(R^{L, n}, R^{G, n}\right)=-2 V\left(R^{L, n}, R^{G, n}\right) \\
&+\frac{2 \delta}{A^{n}} \sum_{i, j \in \mathcal{A}^{n}}\left|r^{L, n}(i, j)+r^{G, n}(i, j)\right|^{+} \\
& \times\left[U_{L}^{n}\left(j, \boldsymbol{\nu}^{-n}\right)+U_{G, t}^{n}(j)\right. \\
&\left.-\left[U_{L}^{n}\left(i, \boldsymbol{\nu}^{-n}\right)+U_{G, t}^{n}(i)\right]\right] .
\end{aligned}
$$

Finally, noting that the payoff function $U_{L}^{n}\left(\cdot, \boldsymbol{\nu}^{-n}\right)+U_{G, t}^{n}(\cdot)$ is bounded, one can find a constant $\alpha$ such that

$$
\begin{aligned}
\frac{d}{d t} V\left(R^{L, n}, R^{G, n}\right) \leq & \frac{2 \alpha \delta}{A^{n}} \sum_{i, j \in \mathcal{A}^{n}}\left|r^{L, n}(i, j)+r^{G, n}(i, j)\right|^{+} \\
& -2 V\left(R^{L, n}, R^{G, n}\right) .
\end{aligned}
$$


In (B.30), $\alpha>0$ depends on the differences in payoffs for different actions $i$ and $j$ and can be specified based on the particular choice of the payoff function. In this lemma, however, we only require existence of such a bound, which is guaranteed since the payoff functions are bounded.

Now, assuming $\left|r^{L, n}(i, j)+r^{G, n}(i, j)\right|^{+}>\epsilon>0$ for all $i, j \in \mathcal{A}^{n}$, one can choose $\delta$ small enough such that

$$
\frac{d}{d t} V\left(R^{L, n}, R^{G, n}\right) \leq-V\left(R^{L, n}, R^{G, n}\right)
$$

This proves global asymptotic stability of the limit system (5.48) and

$$
\lim _{t \rightarrow \infty} \operatorname{dist}\left[R^{L, n}+R^{G, n}, \mathbb{R}^{-}\right]=0 .
$$

Case III: Define the Lyapunov function

$$
\begin{aligned}
V\left(\mathbf{R}^{s}\right) & =\left[\operatorname{dist}\left[\mathbf{R}^{s}, \mathbb{R}^{-}\right]\right]^{2} \\
& =\sum_{k \in C^{s}} \sum_{i, j \in \mathcal{A}^{n}}\left[\left|r^{n}(i, j)\right|^{+}\right]^{2} .
\end{aligned}
$$

Taking the time-derivative of (B.33) and using a similar argument to Lemma B.1, we obtain

$$
\frac{d}{d t} V\left(\mathbf{R}^{s}\right)=\sum_{k \in C^{s}} \sum_{i, j \in \mathcal{A}^{n}}\left|r^{n}(i, j)\right|^{+}\left[\sum_{l \in N_{c}^{n}} q_{n l}^{s} r^{l}(i, j)-r^{n}(i, j)\right]
$$

Recall that $\mathbf{R}^{s}$ represents the global regret matrix for social group $C^{s}$ rearranged into a vector. We consider the following cases:

(i) $\left|\mathbf{R}^{s}\right|^{+}>0$. This is the worst case scenario where all agents' regrets for switching from action $i$ to $j$ is positive. It is straightforward to rewrite (B.34) as follows

$$
\frac{d}{d t} V\left(\mathbf{R}^{s}\right)=\left[\left|\mathbf{R}^{s}\right|^{+}\right]^{\prime}\left(\mathbf{Q}^{s}-I\right) \mathbf{R}^{s}
$$

Noting that, when $\left|\mathbf{R}^{s}\right|^{+}>0,\left|\mathbf{R}^{s}\right|^{+}=\mathbf{R}^{s}$, we obtaine

$$
\begin{aligned}
\frac{d}{d t} V\left(\mathbf{R}^{s}\right) & =\left[\mathbf{R}^{s}\right]^{\prime}\left(\mathbf{Q}^{s}-I\right) \mathbf{R}^{s} \\
& =\left[\mathbf{R}^{s}\right]^{\prime} \mathbf{Q}^{s} \mathbf{R}^{s}-\left[\mathbf{R}^{s}\right]^{\prime} \mathbf{R}^{s}
\end{aligned}
$$


Since $\mathbf{Q}^{s} \mathbf{1}=\mathbf{0}$, it is weakly diagonally dominant. Since $\mathbf{Q}^{s}$ is further symmetric, it is well-known from linear algebra that $\mathrm{Q}^{s}$ is negative semi-definite. That is, for all vectors $\mathbf{x} \in \mathbb{R}^{J_{s}}, \mathbf{x}^{T} \mathbf{Q}^{s} \mathbf{x} \leq 0$. This, together with (B.36), concludes $d V\left(\mathbf{R}^{s}\right) / d t<0$.

(ii) $\left|r^{n}(i, j)\right|^{+}>0$ and $\left|r^{l}(i, j)\right|^{+}=0$ for some $l \in C^{s}$. In (B.34), looking at the term

$$
\left|r^{n}(i, j)\right|^{+}\left[\sum_{l \in N_{c}^{n}} q_{n l}^{s} r^{l}(i, j)-r^{n}(i, j)\right]
$$

it can be clearly seen that $r^{l}(i, j)<0$ actually helps pulling the regrets to the negative orthant more forcefully since $c_{n l}^{s} r^{l}(i, j)-r^{n}(i, j)<0$. This concludes the limit system (5.52) is globally asymptotically stable and (5.54) holds.

\section{B.3 Proof of Theorem 5.4.6}

The proof relies on how the "regret" measure is defined. For brevity, we present the proof for "Case I". Similar argument can be used to prove this result for the other cases. Recall, for $t \in[k \varepsilon,(k+1) \varepsilon), r^{n, \varepsilon}(i, j)(t)=r_{k}^{n}(i, j)$; hence, substituting (5.11) for $r_{k}^{n}(i, j)$ on the r.h.s., we obtain

$$
\begin{aligned}
r^{n, \varepsilon}(i, j)(t) & =\varepsilon \sum_{\tau \leq k}(1-\varepsilon)^{k-\tau}\left[U^{n}\left(j, \mathbf{a}^{-n}(\tau)\right)-U^{n}(\mathbf{a}(\tau))\right] I\left\{\mathbf{a}_{\tau}^{n}=i\right\} \\
& =\sum_{\mathbf{a}^{-n}} z^{n, \varepsilon}\left(i, \mathbf{a}^{-n}\right)(t)\left[U^{n}\left(j, \mathbf{a}^{-n}\right)-U^{n}\left(i, \mathbf{a}^{-n}\right)\right]
\end{aligned}
$$

where $z^{n, \varepsilon}\left(i, \mathbf{a}^{-n}\right)(t)$ denotes the interpolated empirical distribution of agent $n$ choosing action $i$ and the rest playing $\mathbf{a}^{-n}$. On any convergent subsequence $\left\{\boldsymbol{z}_{\underline{k}}\right\}_{\underline{k} \geq 0} \rightarrow \boldsymbol{\pi}$, with slight abuse of notation, let $\boldsymbol{z}^{\varepsilon}(t)=\boldsymbol{z}_{\underline{k}}$ and $R^{n, \varepsilon}(t)=R_{\underline{k}}^{n}$ for $t \in[\underline{k} \varepsilon, \underline{k} \varepsilon+\varepsilon)$. Then,

$$
\lim _{t \rightarrow \infty} r^{n, \varepsilon}(i, j)(t)=\sum_{\mathbf{a}^{-n}} \pi^{n}\left(i, \mathbf{a}^{-n}\right)\left[U^{n}\left(j, \mathbf{a}^{-n}\right)-U^{n}\left(i, \mathbf{a}^{-n}\right)\right]
$$

where $\pi^{n}\left(i, \mathbf{a}^{-n}\right)$ denotes the probability of agent $n$ choosing action $i$ and the rest playing $\mathbf{a}^{-n}$. Next, combining (B.39) with (5.55) in Corollary 5.1, and comparing with (5.8) in Definition 5.3, the desired result follows.

To prove (5.56) for "Case II," one needs to look at the combined regrets $r^{L, n, \varepsilon}(i, j)(t)+r^{G, n, \varepsilon}(i, j)(t)$ instead. We first prove that the sequence $\left\{r_{k}^{G, n}(i, j)\right\}$ 
is an unbiased estimator of the sequence of regrets if agents were to observe the action profile of those outside their social group. The rest of the proof is similar to the above proof. The interested reader is referred to [102, Sec. IV] for the detailed proof. 


\section{Bibliography}

[1] D. Acemoglu, M. Dahleh, I. Lobel, and A. Ozdaglar. Bayesian learning in social networks. Working Paper 14040, National Bureau of Economic Research, May 2008.

[2] D. Acemoglu and A. Ozdaglar. Opinion dynamics and learning in social networks. Dynamic Games and Applications, 1(1):3-49, Mar. 2011.

[3] L. Adamic and E. Adar. How to search a social network. Social Networks, 27(3):187-203, Jul. 2005.

[4] G. Adomavicius and A. Tuzhilin. Toward the next generation of recommender systems: A survey of the state-of-the-art and possible extensions. IEEE Transactions on Knowledge and Data Engineering, 17(6):734-749, Jun. 2005.

[5] C. C. Aggarwal and T. Abdelzaher. Integrating sensors and social networks. Social Network Data Analytics, pages 397-412, 2011.

[6] Y.-Y. Ahn, S. Han, H. Kwak, S. Moon, and H. Jeong. Analysis of topological characteristics of huge online social networking services. In Proceedings of 16th International Conference on World Wide Web, pages 835-844, Banff, AB, Canada, May 2007.

[7] M. Albakour, C. Macdonald, and I. Ounis. Identifying local events by using microblogs as social sensors. In Proceedings of 10th Conference on Open Research Areas in Information Retrieval, pages 173-180, Lisbon, Portugal, May 2013.

[8] R. Albert and A.-L. Barabási. Statistical mechanics of complex networks. Reviews of Modern Physics, 74(1):47-97, Jan. 2002. 
[9] D. Aldous. Interacting particle systems as stochastic social dynamics. Bernoulli, 19(4):1122-1149, 2013.

[10] R. Amir. Supermodularity and complementarity in economics: An elementary survey. Southern Economic Journal, 71(3):636-660, 2005.

[11] C. Anderson and G. J. Kilduff. Why do dominant personalities attain influence in face-to-face groups? the competence-signaling effects of trait dominance. Journal of Personality and Social Psychology, 96(2):491-503, Feb. 2009.

[12] G. M. Angeletos, C. Hellwig, and A. Pavan. Dynamic global games of regime change: Learning, multiplicity, and the timing of attacks. Econometrica, 75(3):711-756, May 2007.

[13] S. Asur and B. A. Huberman. Predicting the future with social media. In 2010 IEEE/WIC/ACM International Conference on Web Intelligence and Intelligent Agent Technology, volume 1, pages 492-499, Toronto, ON, Canada, 2010.

[14] J. P. Aubin. Dynamic Economic Theory: A Viability Approach, volume 174. Springer-Verlag, 1997.

[15] J.-Y. Audibert, S. Bubeck, and R. Munos. Best arm identification in multiarmed bandits. In Proceeding of 23th Conference on Learning Theory, pages 41-53, Haifa, Israel, Jun. 2010.

[16] P. Auer, N. Cesa-Bianchi, and P. Fischer. Finite-time analysis of the multiarmed bandit problem. Machine Learning, 47(2-3):235-256, May 2002.

[17] P. Auer, N. Cesa-Bianchi, Y. Freund, and R. E. Schapire. Gambling in a rigged casino: The adversarial multi-armed bandit problem. In Proceedings of 36th Annual Symposium on Foundations of Computer Science, pages 322-331, Milwaukee, WI, Oct. 1995.

[18] R. J. Aumann. Subjectivity and correlation in randomized strategies. Journal of Mathematical Economics, 1(1):67-96, Mar. 1974.

[19] R. J. Aumann. Agreeing to disagree. The Annals of Statistics, 4(6):12361239, Nov. 1976. 
[20] R. J. Aumann. Correlated equilibrium as an expression of bayesian rationality. Econometrica, 55(1):1-18, Jan. 1987.

[21] M. Avellaneda and S. Stoikov. High-frequency trading in a limit order book. Quantitative Finance, 8(3):217-224, 2008.

[22] V. Bala and S. Goyal. A noncooperative model of network formation. Econometrica, 68(5):1181-1229, Sep. 2000.

[23] A. Banerjee. A simple model of herd behavior. Quaterly Journal of Economics, 107(3):797-817, Aug. 1992.

[24] A.-L. Barabási and A. Reka. Emergence of scaling in random networks. Science, 286(5439):509, 1999.

[25] G. Bebek, P. Berenbrink, C. Cooper, T. Friedetzky, J. Nadeau, and S. C. Sahinalp. The degree distribution of the generalized duplication model. Theoretical Computer Science, 369(1-3):239-249, Dec. 2006.

[26] M. Benaïm, J. Hofbauer, and S. Sorin. Stochastic approximations and differential inclusions, part II: applications. Mathematics of Operations Research, 31(4):673-695, 2006.

[27] M. Benaïm and J. Weibull. Deterministic approximation of stochastic evolution in games. Econometrica, 71(3):873-903, May 2003.

[28] N. Bennett. 4 great (free) tools to measure social sentiment and 4 important stats. http://socialmediatoday.com/nick-bennett/287405/4-great-free-toolsmeasure-social-sentiment-and-4-important-stats, Apr. 2011.

[29] A. Benveniste, M. Métivier, and P. P. Priouret. Adaptive algorithms and stochastic approximations. Springer-Verlag, 1990.

[30] A. Benveniste, M. Métivier, and P. Priouret. Adaptive Algorithms and Stochastic Approximations. Springer, 2012.

[31] S. Bikchandani, D. Hirshleifer, and I. Welch. A theory of fads, fashion, custom, and cultural change as information cascades. Journal of Political Economy, 100(5):992-1026, Oct. 1992.

[32] A. Blum and Y. Mansour. From external to internal regret. Journal of Machine Learning Research, 8(6):1307-1324, 2007. 
[33] J. Bollen, H. Mao, and X. Zeng. Twitter mood predicts the stock market. Journal of Computational Science, 2(1):1-8, Mar. 2011.

[34] B. Bollobás, O. Riordan, J. Spencer, and G. Tusnády. The degree sequence of a scale-free random graph process. Random Structures and Algorithms, 18(3):279-290, 2001.

[35] R. Bond, C. Fariss, J. Jones, A. Kramer, C. Marlow, J. Settle, and J. Fowler. A 61-million-person experiment in social influence and political mobilization. Nature, 489:295-298, Sep. 2012.

[36] V. Borkar and P. Varaiya. Asymptotic agreement in distributed estimation. IEEE Transactions on Automatic Control, 27(3):650-655, Jun. 1982.

[37] S. P. Boyd and L. Vandenberghe. Convex Pptimization. Cambridge University Press, New York, NY, 2004.

[38] Y. Bramoullé and R. Kranton. Public goods in networks. Journal of Economic Theory, 135(1):478-494, Jul. 2007.

[39] T. Brehard and V. Krishnamurthy. Optimal data incest removal in Bayesian decentralized estimation over a sensor network. In Proceedings of IEEE International Conference on Acoustics, Speech and Signal Processing, pages 173-176, Honolulu, Hawaii, Apr. 2007.

[40] P. Bremaud. Markov Chains: Gibbs fields, Monte Carlo Simulation, and Queues. Springer-Verlag, 1999.

[41] J. Burke, D. Estrin, M. Hansen, A. Parker, N. Ramanathan, S. Reddy, and M. B. Srivastava. Participatory sensing. In Workshop on World-SensorWeb: Mobile Device Centric Sensor Networks and Applications, Boulder, $\mathrm{CO}, 2006$.

[42] A. Cahn. General procedures leading to correlated equilibria. International Journal of Game Theory, 33(1):21-40, Dec. 2004.

[43] D. S. Callaway, M. E. J. Newman, S. H. Strogatz, and D. J. Watts. Network robustness and fragility: Percolation on random graphs. Physical Review Letters, 85(25):5468-5471, Dec. 2000. 
[44] A. T. Campbell, S. B. Eisenman, N. D. Lane, E. Miluzzo, R. Peterson, H. Lu, X. Hong, X. Zheng, M. Musolesi, K. Fodor, and G. Ahn. The rise of people-centric sensing. IEEE Internet Computing, 12(4):12-21, 2008.

[45] H. Carlsson and E. van Damme. Global games and equilibrium selection. Econometrica, 61(5):989-1018, Sep. 1993.

[46] E. Cartwright and M. Wooders. Correlated equilibrium, conformity and stereotyping in social groups. Becker Friedman Institute for Research in Economics Working Paper 2012-014, 2012.

[47] T. N. Cason and T. Sharma. Recommended play and correlated equilibria: an experimental study. Economic Theory, 33(1):11-27, Oct. 2007.

[48] A. R. Cassandra. Exact and Approximate Algorithms for Partially Observed Markov Decision Process. PhD thesis, Brown University, 1998.

[49] F. S. Cattivelli and A. H. Sayed. Modeling bird flight formations using diffusion adaptation. IEEE Transactions on Signal Processing, 59(5):20382051, May 2011.

[50] C. Chamley. Rational Herds: Economic Models of Social Learning. Cambridge University Press, 2004.

[51] C. Chamley, A. Scaglione, and L. Li. Models for the diffusion of beliefs in social networks: An overview. IEEE Signal Processing Magazine, 30(3):16-29, May 2013.

[52] C. P. Chamley. Rational Herds: Economic Models of Social Learning. Cambridge University Press, 2003.

[53] D. Chatterjee and D. Liberzon. Stability analysis of deterministic and stochastic switched systems via a comparison principle and multiple lyapunov functions. SIAM Journal on Control and Optimization, 45(1):174206, 2006.

[54] D. Chatterjee and D. Liberzon. On stability of randomly switched nonlinear systems. IEEE Transactions on Automatic Control, 52(12):2390-2394, Dec. 2007. 
[55] K. C. Chen, M. Chuang, and H. V. Poor. From technological networks to social networks. IEEE Journal on Selected Areas in Communications, 31(9):548-572, Sept. 2013.

[56] N. Chen. On the approximability of influence in social networks. SIAM Journal on Discrete Mathematics, 23(3):1400-1415, 2009.

[57] W. Chen, C. Wang, and Y. Wang. Scalable influence maximization for prevalent viral marketing in large-scale social networks. In Proceedings of the 16th ACM SIGKDD International Conference on Knowledge Discovery and Data Mining, pages 1029-1038, Washington, DC, Jul. 2010.

[58] W. Chen, Y. Wang, and S. Yang. Efficient influence maximization in social networks. In Proceedings of the 15th ACM SIGKDD International Conference on Knowledge Discovery and Data Mining, pages 199-208, Paris, France, Jul. 2009.

[59] Z. Cheng, J. Caverlee, and K. Lee. You are where you tweet: A contentbased approach to geo-locating twitter users. In Proceedings of 19th ACM International Conference on Information and Knowledge Management, pages 759-768, Toronto, ON, Canada, Oct. 2010.

[60] N. A. Christakis and J. H. Fowler. Social network sensors for early detection of contagious outbreaks. PLoS ONE, 5(9), Sep. 2010.

[61] F. Chung and L. Lu. Complex Graphs and Networks. Conference Board of the Mathematical Sciences, National Science Foundation (U.S.), 2006.

[62] F. Chung, L. Lu, T. G. Dewey, and D. G. Galas. Duplication models for biological networks. Journal of Computational Biology, 10(5):677-687, Oct. 2003.

[63] C. Cooper and A. Frieze. A general model of web graphs. Random Structures and Algorithms, 22(3):311-335, 2003.

[64] R. Corten. Composition and structure of a large online social network in the netherlands. PLoS ONE, 7(4), Apr. 2012.

[65] T. M. Cover and M. E. Hellman. The two-armed-bandit problem with time-invariant finite memory. IEEE Transactions on Information Theory, 16(2):185-195, Mar. 1970. 
[66] A. Dasgupta, R. Kumar, and D. Sivakumar. Social sampling. In Proceedings of 18th ACM SIGKDD International Conference on Knowledge Discovery and Data Mining, pages 235-243, Beijing, China, Aug. 2012.

[67] A. Dhillon and J. F. Mertens. Perfect correlated equilibria. Journal of Economic Theory, 68(2):279-302, Feb. 1996.

[68] A. Doucet, N. Gordon, and V. Krishnamurthy. Particle filters for state estimation of jump Markov linear systems. IEEE Transactions on Signal Processing, 49(3):613-624, Mar. 2001.

[69] X. G. Doukopoulos and G. V. Moustakides. Adaptive power techniques for blind channel estimation in cdma systems. IEEE Transactions on Signal Processing, 53(3):1110-1120, Mar. 2005.

[70] A. Drexl. Scheduling of project networks by job assignment. Management Science, 37(12):1590-1602, Dec. 1991.

[71] A. Drexl and J. Gruenewald. Non-preemptive multi-mode resourceconstrained project scheduling. IIE transactions, 25(5):74-81, 1993.

[72] J. Duffy and N. Feltovich. Correlated equilibria, good and bad: an experimental study. International Economic Review, 51(3):701-721, Aug. 2010.

[73] F. Dufour and P. Bertrand. An image based filter for discrete-time Markovian jump linear systems. Automatica, 32:241-247, Feb. 1996.

[74] R. Durrett. Random Graph Dynamics. Cambridge Series on Statistical and Probabilistic Mathematics. Cambridge University Press, 2007.

[75] N. Eagle and A. Pentland. Reality mining: Sensing complex social systems. Personal and Ubiquitous Computing, 10(4):255-268, May 2006.

[76] D. Easley and J. Kleinberg. Networks, Crowds, and Markets: Reasoning About a Highly Connected World. Cambridge University Press, 2010.

[77] H. Ebel, L.-I. Mielsch, and S. Bornholdt. Scale-free topology of e-mail networks. Physical Review E, 66, Sep. 2002.

[78] R. J. Elliott, L. Aggoun, and J. B. Moore. Hidden Markov Models: Estimation and Control. Springer-Verlag, New York, NY, 1995. 
[79] G. Ellison and D. Fudenberg. Rules of thumb for social learning. Journal of Political Economy, 101(4):612-643, Aug. 1993.

[80] G. Ellison and D. Fudenberg. Word-of-mouth communication and social learning. The Quarterly Journal of Economics, 110(1):93-125, 1995.

[81] Y. Ephraim and N. Merhav. Hidden Markov processes. IEEE Transactions on Information Theory, 48(6):1518-1569, Jun. 2002.

[82] P. Erdös and T. Gallai. Graphs with given degrees of vertices. Mat Lapok, 11:264-274, 1960.

[83] P. Erdös and A. Rényi. On random graphs, I. Publicationes Mathematicae (Debrecen), 6:290-297, 1959.

[84] P. Erdös and A. Rényi. On the evolution of random graphs. Publ. Math. Inst. Hung. Acad. Sci, 5:17-61, 1960.

[85] S. N. Ethier and T. G. Kurtz. Markov Processes-Characterization and Convergence. Wiley, 1986.

[86] S. Eubank, H. Guclu, V. S. Anil Kumar, M. V. Marathe, A. Srinivasan, Z. Toroczkai, and N. Wang. Modelling disease outbreaks in realistic urban social networks. Nature, 429:180-184, May 2004.

[87] R. Evans, V. Krishnamurthy, G. Nair, and L. Sciacca. Networked sensor management and data rate control for tracking maneuvering targets. IEEE Transactions on Signal Processing, 53(6):1979-1991, Jun. 2005.

[88] M. Faloutsos, P. Faloutsos, and C. Faloutsos. On power-law relationships of the internet topology. In Proceedings of the SIGCOMM conference on Applications, technologies, architectures, and protocols for computer communication, pages 251-262, Cambridge, MA, 1999.

[89] F. Forges. An approach to communication equilibria. Econometrica, pages 1375-1385, Nov. 1986.

[90] D. P. Foster and R. V. Vohra. Calibrated learning and correlated equilibrium. Games and Economic Behavior, 21(1):40-55, Oct. 1997.

[91] D. Fudenberg and D. K. Levine. Consistency and cautious fictitious play. Journal of Economic Dynamics and Control, 19(5):1065-1089, 1995. 
[92] D. Fudenberg and D. K. Levine. The Theory of Learning in Games. MIT Press, 1998.

[93] D. Fudenberg and D. K. Levine. Conditional universal consistency. Games and Economic Behavior, 29(1):104-130, Oct. 1999.

[94] D. Fudenberg and D. K. Levine. Learning and equilibrium. Annual Review of Economics, 1:385-420, Jun. 2009.

[95] S. Ganeriwal, L. K. Balzano, and M. B. Srivastava. Reputation-based framework for high integrity sensor networks. ACM Transactions on Sensor Networks, 4(3):15, May 2008.

[96] A. Garcia, S. D. Patek, and K. Sinha. A decentralized approach to discrete optimization via simulation: Application to network flow. Operations Research, 55(4):717-732, 2007.

[97] J. D. Geanakoplos and H. M. Polemarchakis. We can't disagree forever. Journal of Economic Theory, 28(1):192-200, Oct. 1982.

[98] O. N. Gharehshiran, A. Attar, and V. Krishnamurthy. Collaborative subchannel allocation in cognitive LTE femto-cells: A cooperative gametheoretic approach. IEEE Transactions on Communications, 61(1):325334, Jan. 2013.

[99] O. N. Gharehshiran and V. Krishnamurthy. Coalition formation for bearings-only localization in sensor networks - a cooperative game approach. IEEE Transactions on Signal Processing, 58(8):4322-4338, Aug. 2010 .

[100] O. N. Gharehshiran and V. Krishnamurthy. Diffusion based collaborative decision making in noncooperative social network games. In Proceedings of 1st IEEE Global Conference on Signal and Information Processing, Austin, TX, Dec. 2013.

[101] O. N. Gharehshiran, V. Krishnamurthy, and G. Yin. Distributed energyaware diffusion least mean squares: Game-theoretic learning. IEEE Journal of Selected Topics in Signal Processing, 7(5):821-836, Oct. 2013.

[102] O. N. Gharehshiran, V. Krishnamurthy, and G. Yin. Distributed tracking of correlated equilibria in regime switching noncooperative games. IEEE Transactions on Automatic Control, 58(10):2435-2450, Oct. 2013. 
[103] O. N. Gharehshiran, V. Krishnamurthy, and G. Yin. Adaptive search algorithms for discrete stochastic optimization: A smooth best-response approach. arXiv:1402.3354 [math.OC], Feb. 2014.

[104] D. Gillies. Locations of solutions. In Report of Informal Conf. Theory of n-Person Games, Princton University, 1953.

[105] M. Girvan and M. E. J. Newman. Community structure in social and biological networks. Proceedings of the National Academy of Sciences, 99(12):7821-7826, 2002.

[106] S. Goel and M. J. Salganik. Respondent-driven sampling as markov chain Monte Carlo. Statistics in Medicine, 28(17):2209-2229, Jul 2009.

[107] P. Golle, K. Leyton-Brown, I. Mironov, and M. Lillibridge. Incentives for sharing in peer-to-peer networks. In L. Fiege, G. Mühl, and U. Wilhelm, editors, Electronic Commerce, volume 2232 of Lecture Notes in Computer Science, pages 75-87. Springer Berlin Heidelberg, 2001.

[108] M. Granovetter. Threshold models of collective behavior. American Journal of Sociology, 83(6):1420-1443, May 1978.

[109] C. Griffin and A. Squicciarini. Toward a game theoretic model of information release in social media with experimental results. In Proceeding of IEEE Symposium on Security and Privacy Workshops, pages 113-116, San Francisco, CA, May 2012.

[110] E. Gudes, N. Gal-Oz, and A. Grubshtein. Methods for computing trust and reputation while preserving privacy. In Data and Applications Security XXIII, pages 291-298. Springer, 2009.

[111] S. L. Hakimi. On realizability of a set of integers as degrees of the vertices of a linear graph. I. Journal of the Society for Industrial and Applied Mathematics, 10(3):496-506, 1962.

[112] M. J. Halvey and M. T. Keane. Exploring social dynamics in online media sharing. In Proceedings of 16th international conference on World Wide Web, pages 1273-1274, Banff, AB, Canada, May 2007.

[113] M. Hamdi and V. Krishnamurthy. Removal of data incest in multi-agent social learning in social networks. arXiv:1309.6687 [cs.SI], Sep. 2013. 
[114] M. Hamdi, V. Krishnamurthy, and G. Yin. Tracking the empirical distribution of a Markov-modulated duplication-deletion random graph. arXiv:1303.0050 [cs.IT], Feb. 2013.

[115] J. Hannan. Approximation to bayes risk in repeated play. In M. Dresher, A. W. Tucker, and P. Wolfe, editors, Contributions to the Theory of Games, volume 3, pages 97-139. Princeton University Press, 1957.

[116] S. Hart. Adaptive heuristics. Econometrica, 73(5):1401-1430, Sep. 2005.

[117] S. Hart and A. Mas-Colell. A simple adaptive procedure leading to correlated equilibrium. Econometrica, 68(5):1127-1150, Sep. 2000.

[118] S. Hart and A. Mas-Colell. A general class of adaptive strategies. Journal of Economic Theory, 98(1):26-54, May 2001.

[119] S. Hart and A. Mas-Colell. A reinforcement procedure leading to correlated equilibrium. Economics Essays: A Festschrift for Werner Hildenbrand, pages 181-200, Jun. 2001.

[120] S. Hart and A. Mas-Colell. Uncoupled dynamics do not lead to nash equilibrium. American Economic Review, 93(5):1830-1836, Dec. 2003.

[121] S. Hart, A. Mas-Colell, and Y. Babichenko. Simple Adaptive Strategies: From Regret-Matching to Uncoupled Dynamics, volume 4 of World Scientific Series in Economic Theory. World Scientific Publishing, 2013.

[122] Sergiu Hart. Shapley value. In J. Eatwell, M. Milgate, and P. Newman, editors, The New Palgrave: Game Theory, pages 210-216. Macmillan Press, 1989.

[123] V. Havel. A remark on the existence of finite graphs. Časopis Pěst Mat 80:477, 1955.

[124] D. D. Heckathorn. Respondent-driven sampling: A new approach to the study of hidden populations. Social Problems, 44(2):174-199, May 1997.

[125] D. D. Heckathorn. Respondent-driven sampling II: deriving valid population estimates from chain-referral samples of hidden populations. Social Problems, 49(1):11-34, Feb. 2002. 
[126] M. E. Hellman and T. M. Cover. Learning with finite memory. The Annals of Mathematical Statistics, pages 765-782, Jun. 1970.

[127] J. Hofbauer and K. Sigmund. Evolutionary game dynamics. Bulletin of American Mathematical Society, 40(4):479-519, Jul. 2003.

[128] P. Holme, B. J. Kim, C. N. Yoon, and S. K. Han. Attack vulnerability of complex networks. Physical Review E, 65(5), May 2002.

[129] M. Huang, P. E. Caines, and R. P. Malhame. Social optima in mean field LQG control: Centralized and decentralized strategies. IEEE Transactions on Automatic Control, 57(7):1736-1751, Jul. 2012.

[130] B. Ifrach, C. Maglaras, and M. Scarsini. Monopoly pricing in the presence of social learning. NET Institute Working Paper No. 12-01, 2011.

[131] M. O. Jackson. A survey of network formation models: Stability and efficiency. In Gabrielle Demange and Myrna Wooders, editors, Group Formation in Economics: Networks, Clubs, and Coalitions. Cambridge University Press, 2005.

[132] M. O. Jackson. Social and Economic Networks. Princeton University Press, 2008.

[133] H. Jeong, S. P. Mason, A. L. Barabási, and Z. N. Oltvai. Lethality and centrality in protein networks. Nature, 411(6833):41-42, 2001.

[134] D. W. Johnson and F. P. Johnson. Joining together: Group theory and group skills. Prentice-Hall, Englewood Cliffs, NJ, 1991.

[135] E. A. Jorswieck, E. G. Larsson, M. Luise, H. V. Poor, and A. Leshem. Game theory and the frequency selective interference channel. IEEE Journal of Selected Topics in Signal Processing, 6(2):73-75, Apr. 2012.

[136] Y. Kanoria and O. Tamuz. Tractable bayesian social learning on trees. In Proceedings of IEEE International Symposium on Information Theory, pages 2721 -2725, Cambridge, MA, Jul. 2012.

[137] S. Kar, J. M. F. Moura, and K. Ramanan. Distributed parameter estimation in sensor networks: Nonlinear observation models and imperfect communication. IEEE Transactions on Information Theory, 58(6):3575-3605, Jun. 2012. 
[138] L. Karp, I. H. Lee, and R. Mason. A global game with strategic substitutes and complements. Games and Economic Behavior, 60(1):155-175, Jul. 2007.

[139] M. Kearns. Graphical games. In N. Nisan, T. Roughgarden, E. Tardos, and V. V. Vazirani, editors, Algorithmic Game Theory, volume 3, pages 159-180. Cambridge University Press, 2007.

[140] M. Kearns, M. L. Littman, and S. Singh. Graphical models for game theory. In Proceedings of 17th conference on Uncertainty in Artificial intelligence, pages 253-260, Seattle, WA, Aug. 2001.

[141] M. Kearns, S. Suri, and N. Montfort. An experimental study of the coloring problem on human subject networks. Science, 313(5788):824-827, Aug. 2006.

[142] D. Kempe, J. Kleinberg, and É. Tardos. Maximizing the spread of influence through a social network. In Proceedings of the 9th ACM SIGKDD International Conference on Knowledge Discovery and Data Mining, pages 137-146, Washington, DC, Aug 2003.

[143] H. Kim, Z. Toroczkai, P. L. Erdös, I. Miklós, and L. A. Székely. Degreebased graph construction. Journal of Physics A: Mathematical and Theoretical, 42(39), Oct. 2009.

[144] J. Kleinberg. Navigation in a small world. Nature, 406(6798):845-845, Aug. 2000.

[145] J. Kleinberg, R. Kumar, P. Raghavan, S. Rajagopalan, and A. S. Tomkins. The web as a graph: Measurements, models, and methods. In Proceedings of 5 th Annual International Conference on Computing and Combinatorics, pages 1-17, Tokyo, Japan, Jul. 1999.

[146] J. Kleinberg and P. Raghavan. Query incentive networks. In 46th Annual IEEE Symposium on Foundations of Computer Science, pages 132-141, Pittsburgh, PA, Oct. 2005.

[147] I. Konstas, V. Stathopoulos, and J .M. Jose. On social networks and collaborative recommendation. In Proceedings of 32nd international ACM SIGIR conference on Research and development in information retrieval, pages 195-202, Boston, MA, Jul 2009. 
[148] G. Kossinets and D. J. Watts. Empirical analysis of an evolving social network. Science, 311(5757):88-90, Jan. 2006.

[149] B. Kozma and A. Barrat. Consensus formation on adaptive networks. Physical Review E, 77(1):016102, 2008.

[150] P. L. Krapivsky, G. J. Rodgers, and S. Redner. Degree distributions of growing networks. Physical Review Letters, 86(23):5401-5404, Jun. 2001.

[151] V. Krishnamurthy. Decentralized activation in dense sensor networks via global games. IEEE Transactions on Signal Processing, 56(10):49364950, Oct. 2008.

[152] V. Krishnamurthy. Decentralized spectrum access amongst cognitive radios - an interacting multivariate global game-theoretic approach. IEEE Transactions on Signal Processing, 57(10):3999-4013, Oct. 2009.

[153] V. Krishnamurthy. Bayesian sequential detection with phase-distributed change time and nonlinear penalty-a lattice programming POMDP approach. IEEE Transactions on Information Theory, 57(3):7096-7124, Oct. 2011.

[154] V. Krishnamurthy. Quickest detection POMDPs with social learning: Interaction of local and global decision makers. IEEE Transactions on Information Theory, 58(8):5563-5587, 2012.

[155] V. Krishnamurthy. How to schedule measurements of a noisy Markov chain in decision making? IEEE Transactions on Information Theory, 59(9):4440-4461, Jul. 2013.

[156] V. Krishnamurthy and A. Aryan. Quickest detection of market shocks in agent based models of the order book. In Proceedings of 51st IEEE Conference on Decision and Control, pages 1480-1485, Maui, Hawaii, Dec. 2012.

[157] V. Krishnamurthy and D. Djonin. Structured threshold policies for dynamic sensor scheduling-a partially observed Markov decision process approach. IEEE Transactions on Signal Processing, 55(10):4938-4957, Oct. 2007.

[158] V. Krishnamurthy and R. J. Elliott. Exact finite dimensional filters for doubly stochastic auto-regressive processes. IEEE Transactions on Automatic Control, 42(9):1289-1293, Sep. 1997. 
[159] V. Krishnamurthy and R. J. Elliott. Filters for estimating Markov modulated poisson processes and image based tracking. Automatica, 33(5):821-833, May 1997.

[160] V. Krishnamurthy and M. Hamdi. Mis-information removal in social networks: Constrained estimation on dynamic directed acyclic graphs. IEEE Journal of Selected Topics in Signal Processing, 7(2):333-346, Apr. 2013.

[161] V. Krishnamurthy, M. Maskery, and G. Yin. Decentralized adaptive filtering algorithms for sensor activation in an unattended ground sensor network. IEEE Transactions on Signal Processing, 56(12):6086-6101, Dec. 2008.

[162] V. Krishnamurthy and H. V. Poor. Social learning and Bayesian games in multiagent signal processing: How do local and global decision makers interact? IEEE Signal Processing Magazine, 30(3):43-57, May 2013.

[163] V. Krishnamurthy and H. V. Poor. A tutorial on interactive sensing in social networks. IEEE Transactions on Computational Social Systems, 1(1), 2014. (preprint).

[164] V. Krishnamurthy and T. Ryden. Consistent estimation of linear and nonlinear autoregressive models with Markov regime. Journal of Time Series Analysis, 19(3):291-307, May 1998.

[165] H. J. Kushner. Approximation and Weak Convergence Methods for Random Processes with Application to Stochastics Systems Theory. MIT Press, Cambridge, MA, 1984.

[166] H. J. Kushner and A. Shwartz. An invariant measure approach to the convergence of stochastic approximations with state dependent noise. SIAM Journal on Control and Optimization, 22(1):13-27, 1984.

[167] H. J. Kushner and G. Yin. Stochastic Approximation and Recursive Algorithms and Applications, volume 37 of Stochastic Modeling and Applied Probability. Springer-Verlag, 2nd edition, 2003.

[168] H. Kwak, C. Lee, H. Park, and S. Moon. What is twitter, a social network or a news media? In Proceedings of 19th International Conference on World Wide Web, pages 591-600, Raleigh, NC, Apr. 2010. 
[169] P. G. Lange. Publicly private and privately public: Social networking on youtube. Journal of Computer-Mediated Communication, 13(1):361-380, Oct. 2007.

[170] A. Lansky, A. Abdul-Quader, M. Cribbin, T. Hall, T.J. Finlayson, R. Garffin, L. S. Lin, and P. Sullivan. Developing an HIV behavioral survelillance system for injecting drug users: the National HIV Behavioral Surveillance System. Public Health Reports, 122(S1):48-55, 2007.

[171] R. Lee and K. Sumiya. Measuring geographical regularities of crowd behaviors for twitter-based geo-social event detection. In Proceedings of 2 nd ACM SIGSPATIAL International Workshop on Location Based Social Networks, pages 1-10, San Jose, CA, Nov. 2010.

[172] S. Lee. Understanding respondent driven sampling from a total survey error perspective. Survey Practice, 2(6), 2009.

[173] A. Leshem and E. Zehavi. Game theory and the frequency selective interference channel. IEEE Signal Processing Magazine, 26(5):28-40, Sep. 2009.

[174] J. Leskovec. SNAP library. http://snap.stanford.edu/data/index.html.

[175] J. Leskovec and C. Faloutsos. Sampling from large graphs. In Proceedings of 12th ACM SIGKDD International Conference on Knowledge Discovery and Data Mining, pages 631-636, Philadelphia, PA, Aug. 2006.

[176] J. Leskovec, M. McGlohon, C. Faloutsos, N. Glance, and M. Hurst. Cascading behavior in large blog graphs. arXiv:0704.2803 [physics.soc-ph], Apr. 2007.

[177] D. Liberzon. Switching in Systems and Control. Springer, New York, NY, 2003.

[178] E. Lieberman, C. Hauert, and M. A. Nowak. Evolutionary dynamics on graphs. Nature, 433(7023):312-316, Jan. 2005.

[179] L. Liu, E. Yu, and J. Mylopoulos. Security and privacy requirements analysis within a social setting. In Proceedings of 11th IEEE International Requirements Engineering Conference, pages 151-161, Monterey Bay, CA, Sep. 2003. 
[180] I. Lobel, D. Acemoglu, M. Dahleh, and A. E. Ozdaglar. Preliminary results on social learning with partial observations. In Proceedings of 2 nd International Conference on Performance Evaluation Methodolgies and Tools, Nantes, France, 2007.

[181] A. Logothetis and V. Krishnamurthy. Expectation maximization algorithms for MAP estimation of jump Markov linear systems. IEEE Transactions on Signal Processing, 47(8):2139-2156, Aug. 1999.

[182] C. G. Lopes and A. H. Sayed. Diffusion least-mean squares over adaptive networks: Formulation and performance analysis. IEEE Transactions on Signal Processing, 56(7):3122-3136, Jul. 2008.

[183] D. López-Pintado. Contagion and coordination in random networks. International Journal of Game Theory, 34(3):371-381, Oct. 2006.

[184] D. López-Pintado. Diffusion in complex social networks. Games and Economic Behavior, 62(2):573-590, Mar. 2008.

[185] W. S. Lovejoy. On the convexity of policy regions in partially observed systems. Operations Research, 35(4):619-621, 1987.

[186] W. S. Lovejoy. Some monotonicity results for partially observed Markov decision processes. Operations Research, 35(5):736-743, 1987.

[187] M. Luca. Reviews, reputation, and revenue: The case of Yelp.com, Technical Report 12-016. Harvard Business School, 2011.

[188] R. Maben, G. V. Moustakides, and J. S. Baras. Adaptive sampling for linear state estimation. SIAM Journal on Control and Optimization, 50(2):672702, 2012.

[189] E. Malaguti and P. Toth. A survey on vertex coloring problems. International Transactions in Operational Research, 17(1):1-34, Jan. 2010.

[190] M. Malekinejad, L. Johnston, C. Kendall, L. Kerr, M. Rifkin, and G. Rutherford. Using respondent-driven sampling methodology for HIV biological and behavioral surveillance in international settings: a systematic review. IDS and Behavior, 12(S1):105-130, Jul. 2008. 
[191] M. Maskery, V. Krishnamurthy, and Q. Zhao. Decentralized dynamic spectrum access for cognitive radios: cooperative design of a non-cooperative game. IEEE Transactions on Communication, 57(2):459-469, Feb. 2009.

[192] S. Morris and H. S. Shin. Global games: Theory and applications. In M. Dewatripont, L. P. Hansen, and S. J. Turnovsky, editors, Advances in Economic Theory and Econometrics, Theory and Applications, Eighth World Congress, pages 56-114. Cambridge University Press, 2003.

[193] E. Mossel and S. Roch. On the submodularity of influence in social networks. In Proceeding of 39th ACM Symposium on Theory of Computing, pages 128-134, 2007.

[194] H. Moulin. Game Theory in the Social Sciences. MIT Press, Cambridge, MA, 1986.

[195] H. Moulin and J.-P. Vial. Strategically zero-sum games: The class of games whose completely mixed equilibria cannot be improved upon. International Journal of Game Theory, 7(3-4):201-221, 1978.

[196] G. V. Moustakides. Exponential convergence of products of random matrices: Application to adaptive algorithms. International Journal of Adaptive Control and Signal Processing, 12(7):579-597, 1998.

[197] L. Mui. Computational models of trust and reputation: Agents, evolutionary games, and social networks. PhD thesis, MIT, Department of Electrical Enginering and Computer Science, 2002.

[198] K. P. Murphy, Y. Weiss, and M. I. Jordan. Loopy belief propagation for approximate inference: an empirical study. In Proceedings of 15th Conference on Uncertainty in Artificial Intelligence, pages 467-475, 1999.

[199] J. Nash. Non-cooperative games. Annals of Mathematics, 54(2):286-295, Sep. 1951.

[200] R. Nau, S. G. Canovas, and P. Hansen. On the geometry of nash equilibria and correlated equilibria. International Journal of Game Theory, 32(4):443-453, Aug. 2004.

[201] M. E. J. Newman. The structure of scientific collaboration networks. Proceedings of the National Academy of Sciences, 98(2):404-409, 2001. 
[202] M. E. J. Newman. Assortative mixing in networks. Physical Review Letters, 89, Oct. 2002.

[203] M. E. J. Newman. The structure and function of complex networks. SIAM Review, 45(2):167-256, 2003.

[204] M. E. J. Newman, D. J. Watts, and S. H. Strogatz. Random graph models of social networks. Proceedings of the National Academy of Sciences, 99(S1):2566-2572, 2002.

[205] N. Nisan. Algorithmic Game Theory. Cambridge University Press, 2007.

[206] M. J. Osborne and A. Rubinstein. A Course in Game Theory. MIT Press, Cambridge, MA, 1994.

[207] M. Ottaviani and P. Sørensen. Information aggregation in debate: Who should speak first? Journal of Public Economics, 81(3):393-421, Sep. 2001.

[208] B. Pang and L. Lee. Opinion mining and sentiment analysis. Foundations and Trends in Information Retrieval, 2(1-2):1-135, 2008.

[209] C. H. Papadimitriou and J.N. Tsitsiklis. The compexity of Markov decision processes. Mathematics of Operations Research, 12(3):441-450, 1987.

[210] J. Park and M. Van der Schaar. A game theoretic analysis of incentives in content production and sharing over peer-to-peer networks. IEEE Journal of Selected Topics in Signal Processing, 4(4):704-717, Aug. 2010.

[211] R. Pastor-Satorras, E. Smith, and R. V. Solé. Evolving protein interaction networks through gene duplication. Journal of Theoretical Biology, 222(2):199-210, May 2003.

[212] R. Pastor-Satorras and A. Vespignani. Epidemic spreading in scale-free networks. Physical Review Letters, 86(14):3200, 2001.

[213] J. Pearl. Fusion, propagation, and structuring in belief networks. Artificial Intelligence, 29(3):241-288, Sep. 1986.

[214] H. V. Poor and O. Hadjiliadis. Quickest Detection. Cambridge University Press, 2008. 
[215] J. B. Predd, S. R. Kulkarni, and H. V. Poor. A collaborative training algorithm for distributed learning. IEEE Transactions on Information Theory, 55(4):1856-1871, Apr. 2009.

[216] D. Ray. A Game-Theoretic Perspective on Coalition Formation. Oxford University Press, 2008.

[217] D. Ray and R. Vohra. Coalition formation. In P. Young and S. Zamir, editors, Handbook of Game Theory with Economic Applications, volume 4. Elsevier, 2013.

[218] U. Rieder. Structural results for partially observed control models. Methods and Models of Operations Research, 35(6):473-490, 1991.

[219] J. Robinson. An iterative method of solving a game. Annals of Mathematics, 54(2):296-301, Sep. 1951.

[220] M. Rogers, C. Chapman, and V. Giotsas. Measuring the diffusion of marketing messages across a social network. Journal of Direct, Data and Digital Marketing Practice, 14:97-130, Nov. 2012.

[221] A. Rosi, M. Mamei, F. Zambonelli, S. Dobson, G. Stevenson, and Y. Juan. Social sensors and pervasive services: Approaches and perspectives. In Proceedings of IEEE International Conference on Pervasive Computing and Communications Workshops, pages 525-530, Seattle, WA, Mar. 2011.

[222] T. Sakaki, M. Okazaki, and Y. Matsuo. Earthquake shakes twitter users: Real-time event detection by social sensors. In Proceedings of 19th International Conference on World Wide Web, pages 851-860, Raleigh, NC, Apr. 2010.

[223] W. H. Sandholm. Population Games and Evolutionary Dynamics. MIT Press, Cambridge, MA, 2010.

[224] A. H. Sayed. Fundamentals of Adaptive Filtering. Wiley, New York, 2003.

[225] A. H. Sayed. Diffusion adaptation over networks. In R. Chellapa and S. Theodoridis, editors, Academic Press Library in Signal Processing, volume 3, pages 323-454. Academic Press, Elsevier, 2013.

[226] A. H. Sayed. Adaptive networks. Proceedings of the IEEE, 102(4):460497, Apr. 2014. 
[227] A. H. Sayed, S. Barbarossa, S. Theodoridis, and I. Yamada. Introduction to the issue on adaptation and learning over complex networks. IEEE Journal of Selected Topics in Signal Processing, 7(2):161-162, 2013.

[228] R. Selten. A note on evolutionarily stable strategies in asymmetric animal conflicts. Journal of Theoretical Biology, 84(1):93-101, May 1980.

[229] L. S. Shapley. Open questions. In Report of Informal Conf. Theory of n-Person Games, Princton University, 1953.

[230] L. S. Shapley. A value for $n$-person games. In H. W. Kuhn and A. W. Tucker, editors, Contributions to the Theory of Games: Vol. II, Annals of Mathematics Studies v. 28, pages 307-317. Princeton University Press, 1953.

[231] A. N. Shiryaev. On optimum methods in quickest detection problems. Theory of Probability and its Applications, 8(1):22-46, 1963.

[232] J. Shrager, T. Hogg, and B. A. Huberman. A graph-dynamic model of the power law of practice and the problem-solving fan-effect. Science, 242(4877):414-416, 1988.

[233] B. Skyrms and R. Pemantle. A dynamic model of social network formation. In T. Gross and H. Sayama, editors, Adaptive Networks: Theorey, Methods and Applications, Understanding Complex Systems, pages 231251. Springer Berlin Heidelberg, 2009.

[234] M. Slikker and A. Van den Nouweland. Social and Economic Networks in Cooperative Game Theory, volume 27 of Theory and Decision Library C. Springer, 2001.

[235] L. Smith and P. Sorensen. Informational herding and optimal experimentation. Economics Papers 139, Economics Group, Nuffield College, University of Oxford, 1997.

[236] S. H. Strogatz. Exploring complex networks. Nature, 410(6825):268, 2001.

[237] E. Sun, I. Rosenn, C. Marlow, and T. M. Lento. Gesundheit! modeling contagion through facebook news feed. In Proceedings of 3rd International AAAI Conference on Weblogs and Social Media, San Jose, CA, May 2009. 
[238] J. Surowiecki. The Wisdom of Crowds. Anchor, 2005.

[239] D. D. Sworder and R. G. Hutchins. Image-enhanced tracking. IEEE Transactions on Aerospace and Electronic Systems, 25(5):701-709, Sep. 1989.

[240] A. Tahbaz-Salehi and A. Jadbabaie. Consensus over ergodic stationary graph processes. IEEE Transactions on Automatic Control, 55(1):225-230, Jan. 2010.

[241] C. Tantipathananandh, T. Berger-Wolf, and D. Kempe. A framework for community identification in dynamic social networks. In Proceedings of 13th ACM SIGKDD Intlernational Conference on Knowledge Discovery and Data Mining, pages 717-726, San Jose, CA, 2007.

[242] R. Taylor. Contrained switchings in graphs. In K. L. McAvaney, editor, Combinatorial Mathematics VIII, volume 884 of Lecture Notes in Mathematics, pages 314-336. Springer Berlin Heidelberg, 1981.

[243] D. M. Topkis. Supermodularity and Complementarity. Princeton University Press, 1998.

[244] A. Tripathi and S. Vijay. A note on a theorem of erdös and gallai. Discrete Mathematics, 265(1-3):417-420, Apr. 2003.

[245] M. Trusov, A. V. Bodapati, and R. E. Bucklin. Determining influentual users in internet social networks. Journal of Marketing Research, XLVII:643-658, Aug. 2010.

[246] S.-Y. Tu and A. H. Sayed. Mobile adaptive networks. IEEE Journal of Selected Topics in Signal Processing, 5(4):649-664, Aug. 2011.

[247] B. Tuttle. Fact-checking the crowds: How to get the most out of hotelreview sites. Time magazine, Jul. 2013.

[248] V. Tzoumas, C. Amanatidis, and E. Markakis. A game-theoretic analysis of a competitive diffusion process over social networks. In Internet and Network Economics, volume 7695, pages 1-14. Springer, 2012.

[249] F. Vega-Redendo. Complex Social Networks. Economic Society Monographs. Cambridge University Press, 2007. 
[250] A. Wagner. The yeast protein interaction network evolves rapidly and contains few redundant duplicate genes. Molecular Biology and Evolution, 18(7):1283-1292, 2001.

[251] G. Wang, S. R. Kulkarni, H. V. Poor, and D. Osherson. Aggregating large sets of probabilistic forecasts by weighted coherent adjustment. Decision Analysis, 8(2):128-144, Jun. 2011.

[252] T. Wang and H. Krim. Statistical classification of social networks. In Proceedings of IEEE International Conference on Acoustics, Speech and Signal Processing, pages 3977-3980, Kyoto, Japan, Mar. 2012.

[253] T. Wang, H. Krim, and Y. Viniotis. A generalized Markov graph model: Application to social network analysis. IEEE journal of Selected Topics in Signal Processing, 7(2):318-332, Apr. 2013.

[254] D. J. Watts. Networks, dynamics, and the small-world phenomenon. American Journal of Sociology, 105(2):493-527, Sep. 1999.

[255] D. J. Watts and S. H. Strogatz. Collective dynamics of 'small-world' networks. Nature, 393(6684):440-442, Jun. 1998.

[256] K. Wickre. Twitter blog. https://blog.twitter.com/2013/celebratingtwitter7, Mar. 2013.

[257] J. Xu and M. Van der Schaar. Social norm design for information exchange systems with limited observations. IEEE Journal of Selected Areas in Communication, 30(11):2126-2135, Dec. 2012.

[258] J. S. Yedidia, W. T. Freeman, and Y. Weiss. Constructing free-energy approximations and generalized belief propagation algorithms. IEEE Transactions on Information Theory, 51(7):2282-2312, Jul. 2005.

[259] G. Yin and V. Krishnamurthy. LMS algorithms for tracking slow Markov chains with applications to hidden Markov estimation and adaptive multiuser detection. IEEE Transactions on Information Theory, 51(7):24752490, Jul. 2005.

[260] G. Yin, V. Krishnamurthy, and C. Ion. Regime switching stochastic approximation algorithms with application to adaptive discrete stochastic optimization. SIAM Journal on Optimization, 14(4):1187-1215, 2004. 
[261] G. Yin and Q. Zhang. Continuous-time Markov Chains and Applications: A Singular Perturbation Approach. Springer, 1998.

[262] G. Yin and Q. Zhang. Discrete-Time Markov Chains: Two-Time-Scale Methods and Applications, volume 55 of Applications of Mathematics. Srpinger-Verlag, New York, NY, 2005.

[263] G. Yin and C. Zhu. Hybrid Switching Diffusions: Properties and Applications, volume 63 of Stochastic Modeling and Applied Probability. Springer, 2010 .

[264] W. Zame, J. Xu, and M. Van der Schaar. Winning the lottery: Learning perfect coordination with minimal feedback. IEEE Journal of Selected Topics in Signal Processing, 7(5):846-857, Oct. 2013.

[265] Y. Zhang and M. Van der Schaar. Robust reputation protocol design for online communities: A stochastic stability analysis. IEEE Journal of Selected Topics in Signal Processing, 7(5):907-920, Oct. 2013.

[266] Y. Zhang and M. Van der Schaar. Strategic networks: Information dissemination and link formation among self-interested agents. IEEE Journal of Selected Areas in Communication, 31(6):1115-1123, Jun. 2013.

[267] H. V. Zhao, W. S. Lin, and K. J. R. Liu. Behavior dynamics in mediasharing social networks. Cambridge University Press, 2011.

[268] Q. Zhao, E. K. P. Chong, B. Krishnamachari, A. Leshem, S. Meyn, and V. V. Veeravalli. Introduction to the issue on learning-based decision making in dynamic systems under uncertainty. IEEE Journal of Selected Topics in Signal Processing, 7(5):743-745, Oct. 2013.

[269] K. Zhu and J. P. Weyant. Strategic decisions of new technology adoption under asymmetric information: A game-theoretic model. Decision Sciences, 34(4):643-675, Nov. 2003.

[270] A. M. Zoubir, V. Krishnamurthy, and A. H. Sayed. Signal processing theory and methods [in the spotlight]. IEEE Signal Processing Magazine, 28(5):152-156, Sep. 2011. 


\section{Index}

correlated equilibrium, 101

data incest, 25, 30, 78

degree distribution, 46, 53

expected degree distribution, 54

degree sequence, 62

differential inclusion, 119, 121-123, 156

diffusion least mean square, 130

energy-aware diffusion LMS, 132, 135

Erdös-Gallai theorem, 63

game, 94

cooperative game, 140

global game, 43

non-cooperative game, 97

regime-switching game, 125, 126

repeated game, 98

simultaneous move game, 98

giant component, 46

graph

connectivity graph, 104

duplication-deletion graph, 50

graph construction, 62

Markov-modulated random graph, 52

mean field dynamics, 83

half-edge, 63

Havel-Hakimi theorem, 63 herding, 21

information cascade, 21

learning

adaptive learning, 94, 102, 105

reinforcement learning, 95, 112

Lyapunov function, 149, 161, 162, 164

Markov chain, 50

Markovian switching, 62, 125

matrix

adjacency matrix, 27, 62

covariance matrix, 62

generator matrix, 53, 125, 128

transition matrix, 53, 125, 157

transitive closure matrix, 28, 35

weight matrix, 114

mean field dynamics, 72, 78, 84, 89

mean square error, 60

mixed strategy, 98

Nash equilibrium, 100

nonlinear filtering, 87, 89

hidden Markov model filter, 18, 39,

89

social learning filter, 17-20, 39

online reputation, 25,30

ordinal decisions, 36

ordinary differential equation, 60, 122,

131 
Partially Observed Markov Decision Process (POMDP), 22, 23, 39

power law, 49, 55

power law exponent, 55, 57

privacy, 52

regret-matching procedure, 102, 105, 106

respondent-driven sampling, 81

searchability, 47

sentiment, 72, 73, 79, 86, 92

social group, 108

homogeneous social group, 112 social influence constraint, 30

social learning, 17, 25

social sampling, 80

social sensor, 46, 49, 58

state of nature, 48

stochastic approximation, 59, 131

adaptive filtering, 108

supermodularity, 23, 24

tightness, 153, 158

tracking error, 61

weak convergence, $60,86,118,119,153$

wisdom of crowds, 42 


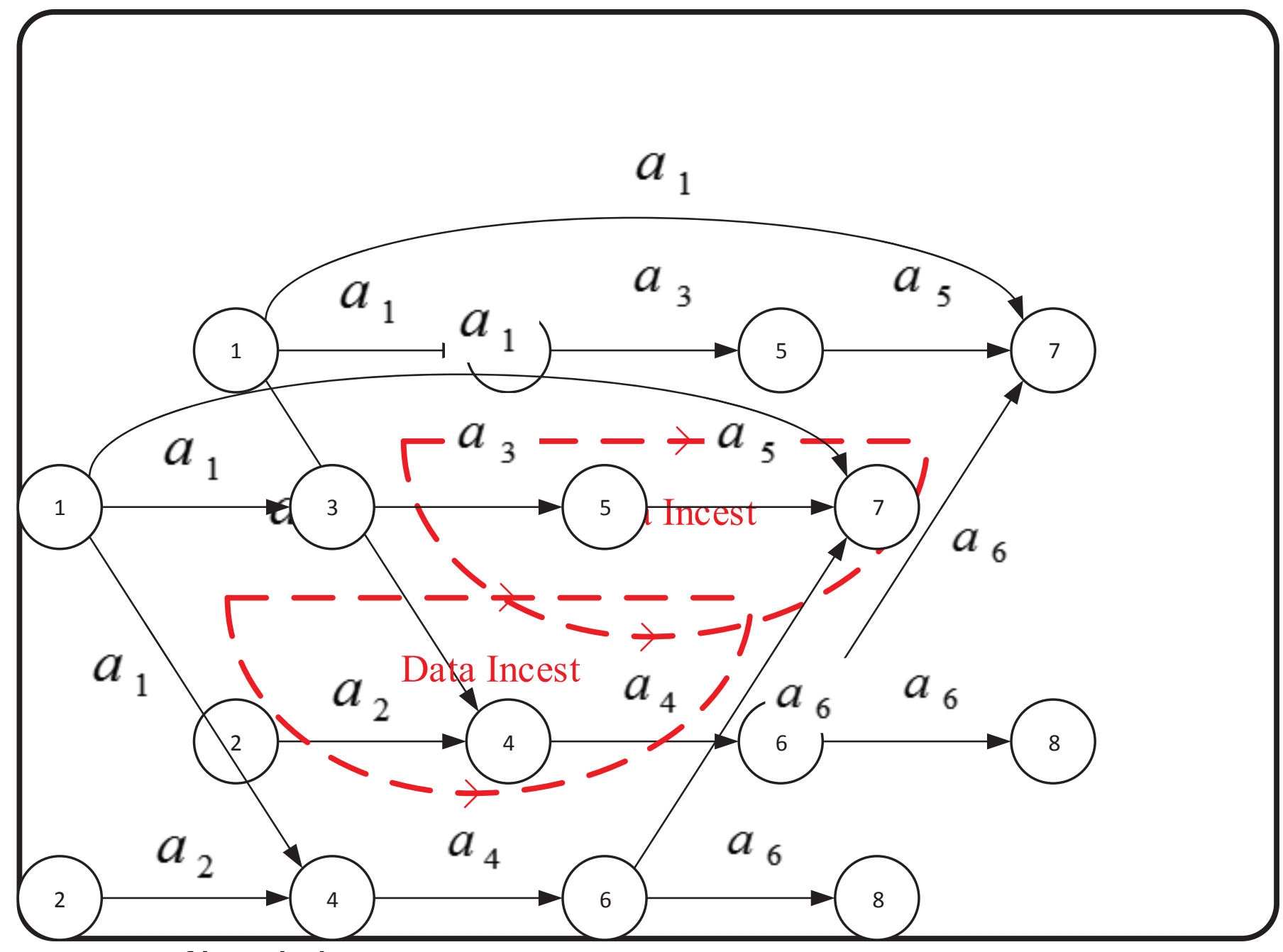

the essence of knowledge 


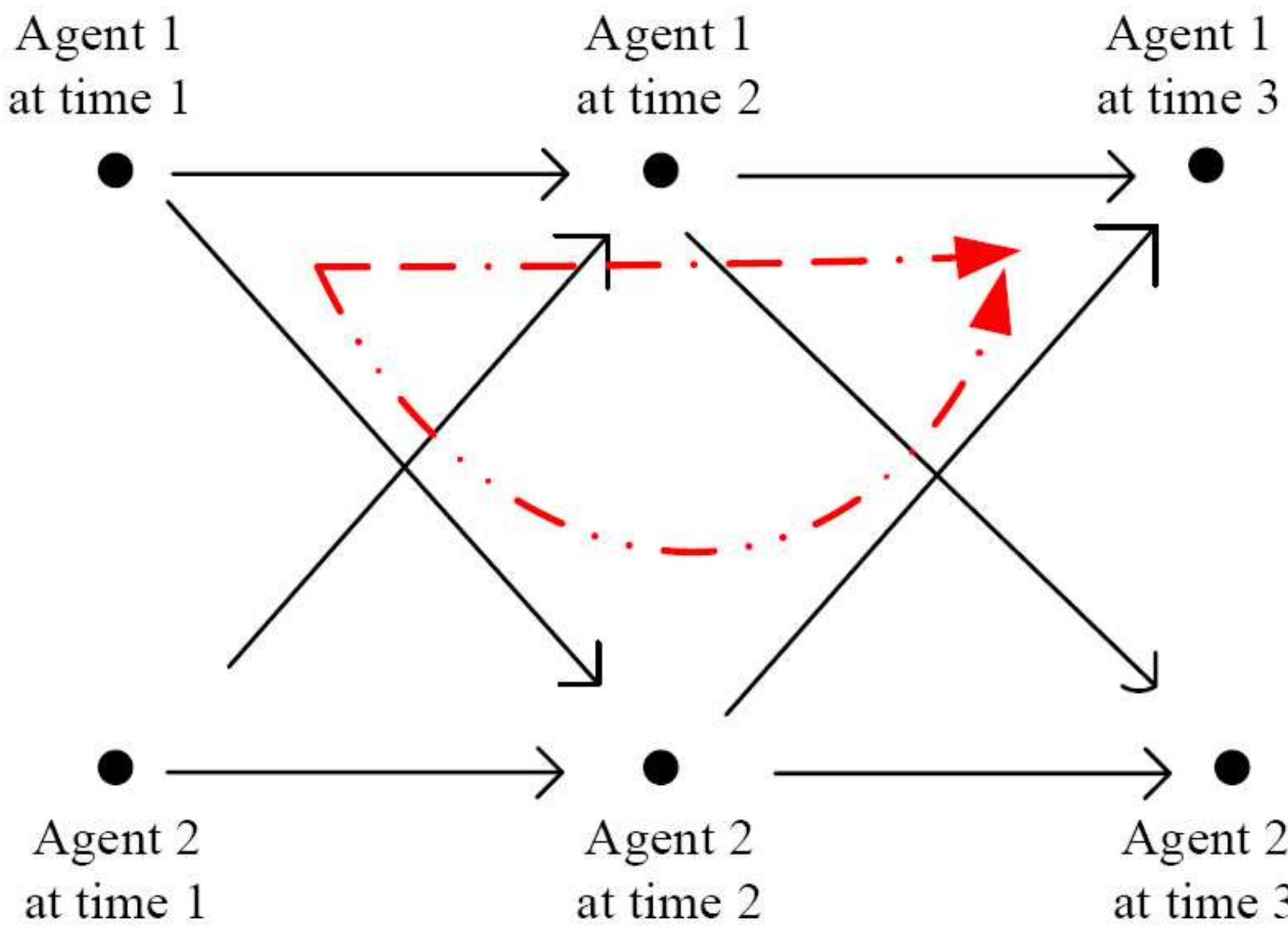

